\title{
Boron Lewis Acid-Catalyzed Regioselective Hydrothiolation of Conjugated Dienes with Thiols
}

Gautam Kumar $^{\dagger}$, Zheng-Wang Qu ${ }^{\ddagger *}$, Soumen Ghosh ${ }^{\dagger}$, Stefan Grimme ${ }^{\ddagger}$ and Indranil Chatterjee ${ }^{{ }_{*}}$

${ }^{\dagger}$ Department of Chemistry, Indian Institute of Technology Ropar

Ropar, Punjab-140001, India

Indranil.chatterjee@iitrpr.ac.in

${ }^{\ddagger}$ Mulliken Center for Theoretical Chemistry,

Institut für Physikalische und Theoretische Chemie, Universität Bonn

Beringstraße 4, D-53115, Bonn (Germany)

qu@thch.uni-bonn.de

\section{Supporting Information}




\section{Table of Contents}

1.0 General Information $\quad$ S4

2.0 Optimization of Reaction Conditions for Hydrothiolation $\quad$ S5

3.0 Possible Isomers for the Hydrothiolation of substituted 1,3-diene S5

4.0 List of the Dienes and Thiols Used $\quad$ S6

5.0 General Procedure for the Reaction of 1,3-Dienes and Thiols (GP) S7

6.0 Experimental Details for the Synthesized Compounds $\quad$ S8

$6.1 \quad(E)$-phenyl(4-phenylbut-3-en-2-yl)sulfane (3) S8

$6.2(E)$-(4-chlorophenyl)(4-phenylbut-3-en-2-yl)sulfane (4) S8

6.3 (E)-(4-methoxyphenyl)(4-phenylbut-3-en-2-yl)sulfane (5) S9

$6.4 \quad(E)$-naphthalen-1-yl(4-phenylbut-3-en-2-yl)sulfane (6) S9

$6.5 \quad(E)$-benzyl(4-phenylbut-3-en-2-yl)sulfane (7) S10

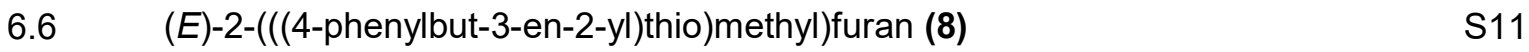

$6.7(E)$-isopentyl(4-phenylbut-3-en-2-yl)sulfane (9) S11

$6.8 \quad(E)$-cyclohexyl(4-phenylbut-3-en-2-yl)sulfane (10) S12

$6.9 \quad(E)$-benzyl 3-((4-phenylbut-3-en-2-yl)thio)propanoate (11) S12

6.10 (E)-(4-phenylbut-3-en-2-yl)(1-phenylpropan-2-yl)sulfane (12) S13

6.11 (E)-3-((4-phenylbut-3-en-2-yl)thio)propane-1-thiol and $((E)-4-p h e n y l b u t-3-e n-2-\quad S 14$ yl)(3-(((E)-5-phenylpent-4-en-2-yl)thio)propyl)sulfane (13 + 13')

$6.12 \quad(E)$-phenyl(4-(p-tolyl)but-3-en-2-yl)sulfane (14) S15

$6.13(E)-(4-(4-m e t h o x y p h e n y l) b u t-3-e n-2-y l)($ phenyl)sulfane (15) S16

$6.14 \quad(E)-(4-(4-c h l o r o p h e n y l) b u t-3-e n-2-y l)($ phenyl)sulfane (16) S16

6.15 (E)-2-(3-(phenylthio)but-1-en-1-yl)furan (17) S17

6.16 (E)-(4-(naphthalen-2-yl)but-3-en-2-yl)(phenyl)sulfane and (Z)-(4-(naphthalen- S17 2-yl)but-3-en-2-yl)(phenyl)sulfane (18)

$6.17(E)-(4-(f e r r o c e n e-2-y l) b u t-3-e n-2-y l)($ phenyl)sulfane (19) and (1-(ferrocene-2- S18 yl)butane-1,3-diyl)bis(phenylsulfane) (19')

6.18 oct-3-en-2-yl(phenyl)sulfane, oct-2-en-1-yl(phenyl)sulfane \& oct-3-en-1- S19 $\mathrm{yl}($ phenyl)sulfane $(\mathbf{2 0 + 2 0 ' + 2 0 " )}$

6.19 (4-cyclohexylbut-3-en-2-yl)(phenyl)sulfane, (4-cyclohexylbut-2-en-1- S20 yl)(phenyl)sulfane \& (4-cyclohexylbut-3-en-1-yl)(phenyl)sulfane (21+21'+21")

6.20 phenyl(6-phenylhex-2-en-1-yl)sulfane and phenyl(6-phenylhex-3-en-1- S21 yl)sulfane $(22+22 ')$

$6.21 \quad(E)$-phenyl(4-phenylpent-3-en-2-yl)sulfane (23) S21

6.22 (4,4-diphenylbut-3-en-2-yl)(phenyl)sulfane (24) S22

6.23 (1-cyclohexylidenepropan-2-yl)(phenyl)sulfane (25) S23 
$6.24(E)$-phenyl(1-phenylpent-1-en-3-yl)sulfane (26) S23

$6.25 \quad E)$-phenyl(1-phenyloct-1-en-3-yl)sulfane (27) S24

$6.26 \quad(E)-(2-m e t h y l-4-p h e n y l b u t-3-e n-2-y l)($ phenyl)sulfane (28) S24

6.27 (E)-(3-methyl-1-phenylpent-1-en-3-yl)(phenyl)sulfane (29) S25

6.28 (E)-(3-methyl-1-phenyloct-1-en-3-yl)(phenyl)sulfane (30) S25

6.29 (E)-(2-methyl-1,4-diphenylbut-3-en-2-yl)(phenyl)sulfane (31) S26

6.30 (2-methylbut-2-en-1-yl)(phenyl)sulfane and (3-methylbut-2-en-1- S27 $\mathrm{yl})($ phenyl)sulfane $(32+32 ')$

6.31 (2-ethylidene-6-methylhept-5-en-1-yl)(phenyl)sulfane (33) S27

6.32 Cyclohex-2-en-1-yl(phenyl)sulfane (34) S28

$\begin{array}{lll}7.0 & \text { Gram scale synthesis } & \text { S29 }\end{array}$

8.0 Derivatization of the sulfide to sulfone $\quad$ S29

$8.1 \quad(E)-((4-p h e n y l b u t-3-e n-2-y l)$ sulfonyl)benzene (35) S29

8.2 (3-(phenylsulfonyl)but-1-ene-1,1-diyl)dibenzene (36) S30

8.3 (E)-(2-((4-phenylbut-3-en-2-yl)sulfonyl)propyl)benzene (38) S30

$9.0 \quad$ Hydrodesulfurization of the synthesized sufide $\quad$ S31

$9.1 \quad(E)$-but-1-en-1-ylbenzene and $(E)$-but-2-en-1-ylbenzene $(\mathbf{3 7}+\mathbf{3 7})$

10.0 Deuterium labelling $\quad$ S32

10.1 (E)-phenyl(4-phenylbut-3-en-2-yl)sulfane- $d_{1}(3-d) \quad S 32$

11.0 Time-Dependent ${ }^{1} \mathrm{H}$ NMR Measurement of Model Reaction S33

$12.0 \quad{ }^{11} \mathrm{~B}$ NMR for the Detection of $\mathrm{B}\left(\mathrm{C}_{6} \mathrm{~F}_{5}\right)_{3}$ and Thiophenol Adduct Formation S34

$\begin{array}{lll}13.0 & \text { NMR Spectra } & \text { S35 }\end{array}$

$\begin{array}{lll}14.0 & \text { DFT Calculations } & \text { S78 }\end{array}$

$\begin{array}{lll}14.1 & \text { Computational details } & \text { S78 }\end{array}$

14.2 Figure S1: DFT-computed free energy reaction profiles $\quad$ S80

14.3 Table S2: DFT-computed energies $\quad$ S81

14.4 Table S3: DFT-optimized atomic Cartesian coordinates S83

$\begin{array}{llr}15.0 & \text { References } & \mathbf{S 1 1 9}\end{array}$ 


\section{$1.0 \quad$ General Information}

Reactions were performed inside the argon filled glove box, using oven-dried glassware. Liquids and solutions were transferred with syringes. Solvents used were dried and purified by following standard procedures. Technical grade solvents for extraction or chromatography (ethyl acetate, and petroleum ether) were distilled prior to use. $\mathrm{CDCl}_{3}$ was stored over $4 \AA$ molecular sieves. Used chemicals were purchased from SigmaAldrich, TCl, Alfa-Aesar and Sisco Research Laboratories (SRL) used without further purification. All the liquid chemicals distilled freshly prior to use. Analytical thin-layer chromatography (TLC) was performed on silica gel 60 F254 glass plates from Merck. Flash column chromatography was performed on silica gel $60(40-63 \mu \mathrm{m}, 230-400$ mesh, ASTM) from Merck using the indicated solvents. ${ }^{1} \mathrm{H},{ }^{13} \mathrm{C}$, and ${ }^{11} \mathrm{~B}$ NMR spectra were recorded in $\mathrm{CDCl}_{3}$ or Toluene- $d_{8}$ unless otherwise stated on JEOL JNM ECS-400 instrument. Chemical shifts are reported in parts per million (ppm) and are referenced to the residual solvent resonance as the internal standard $\left(\mathrm{CDCl}_{3}: \delta=7.26 \mathrm{ppm}\right.$ for ${ }^{1} \mathrm{H} \mathrm{NMR}$ and $\mathrm{CDCl}_{3}: \delta=77.16 \mathrm{ppm}$ for ${ }^{13} \mathrm{C} \mathrm{NMR}$; 1,2-Dibromomethane was used as an internal standard to calculate NMR yields. Data are reported as follows: chemical shift, multiplicity ( $b r=$ broad singlet, $s=$ singlet, $d=$ doublet, $d d=$ doublet of doublets, $t=$ triplet, $q=$ quartet, sept $=$ septet, $\mathrm{m}=$ multiplet), coupling constants $(\mathrm{Hz})$, and integration. All the HRMS/MS-MS data were recorded on XEVO G2-XS QTOF. Dienes $\mathbf{1 t}, \mathbf{1} \mathbf{u}, \mathbf{1} \mathbf{v}$ and thiols 2a, 2b, 2c, 2d, 2e, 2f, 2g, 2h, 2k has been obtained from commercial sources. Dienes $\mathbf{1 a - 1 s},{ }^{[\mathrm{S1}-\mathrm{S} 6]}$ and thiols $\mathbf{2 a - d ,} \mathbf{2} \mathbf{i}, \mathbf{2} \mathbf{k}^{\left[{ }^{[57-S 9]}\right.}$ were synthesized according to the procedure in the literature. 


\begin{tabular}{|c|c|c|c|}
\hline Entry & catalyst $(\mathrm{mol} \%)$ & Solvent & Yield $3(\%)^{[a]}$ \\
\hline 1 & $\mathrm{~B}\left(\mathrm{C}_{6} \mathrm{~F}_{5}\right)_{3}(10)$ & Dioxane & $\mathrm{nr}$ \\
\hline 2 & $\mathrm{~B}\left(\mathrm{C}_{6} \mathrm{~F}_{5}\right)_{3}(10)$ & THF & $\mathrm{nr}$ \\
\hline 3 & $\mathrm{~B}\left(\mathrm{C}_{6} \mathrm{~F}_{5}\right)_{3}(10)$ & $\mathrm{EtOH}$ & $\mathrm{nr}$ \\
\hline 4 & $\mathrm{~B}\left(\mathrm{C}_{6} \mathrm{~F}_{5}\right)_{3}(10)$ & $\mathrm{MeNO}_{2}$ & 20 \\
\hline 5 & $\mathrm{~B}\left(\mathrm{C}_{6} \mathrm{~F}_{5}\right)_{3}(10)$ & DCM & 61 \\
\hline 6 & $\mathrm{~B}\left(\mathrm{C}_{6} \mathrm{~F}_{5}\right)_{3}(10)$ & 1,2-difluorobenzene & 64 \\
\hline 7 & $\mathrm{~B}\left(\mathrm{C}_{6} \mathrm{~F}_{5}\right)_{3}(10)$ & Toluene & 99 \\
\hline 8 & $\mathrm{~B}\left(\mathrm{C}_{6} \mathrm{~F}_{5}\right)_{3}(5)$ & Toluene & 99 \\
\hline 9 & $\mathrm{~B}\left(\mathrm{C}_{6} \mathrm{~F}_{5}\right)_{3}(2.5)$ & Toluene & 99 \\
\hline 10 & $B\left(C_{6} F_{5}\right)_{3}(1)$ & Toluene & $>99(98)^{[b]}$ \\
\hline 11 & $\mathrm{~B}\left(\mathrm{C}_{6} \mathrm{~F}_{5}\right)_{3}(0.5)$ & Toluene & 66 \\
\hline 12 & $\mathrm{BF}_{3} \cdot \mathrm{Et}_{2} \mathrm{O}(1)$ & Toluene & $88^{[\mathrm{b}]}$ \\
\hline 13 & $\mathrm{BCl}_{3}(1)^{[\mathrm{c}]}$ & Toluene & $83^{[b]}$ \\
\hline 14 & $\mathrm{Tf}_{2} \mathrm{NH}(1)$ & Toluene & $75^{[\mathrm{b}]}$ \\
\hline 15 & $\mathrm{Cu}(\mathrm{OTf})_{2}(1)$ & Toluene & $78^{[\mathrm{b}]}$ \\
\hline
\end{tabular}

Table S1 [a] Reaction condition $\mathbf{1 a}$ (1.2 equiv) and $\mathbf{2 a}$ (1.0 equiv) stirred in solvent at rt for 2 h. Yield determined by ${ }^{1} \mathrm{H}$ NMR using $\mathrm{CH}_{2} \mathrm{Br}_{2}$ as internal standard. [b] Isolated yield. [c] Used as $1 \mathrm{M}$ solution in $\mathrm{DCM}$. $\mathrm{nr}=$ no reaction.

\subsection{Possible Isomers for the Hydrothiolation of substituted 1,3-diene}

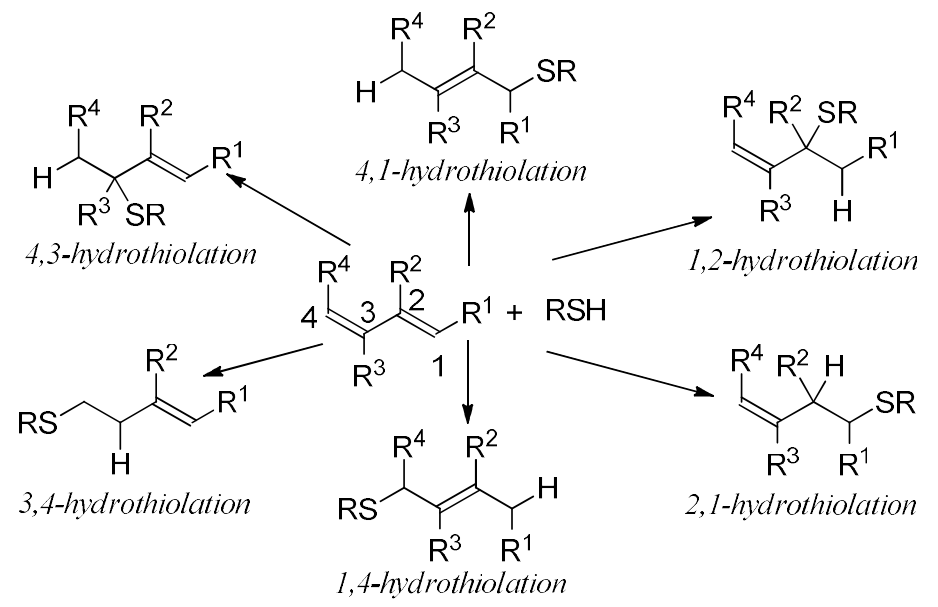




\subsection{List of the Dienes and Thiols Used}

Successful Dienes

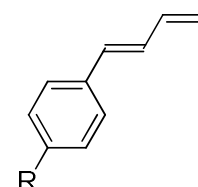

$\mathrm{R}=\mathrm{H}, \mathbf{1} \mathbf{a}$

$\mathrm{R}=\mathrm{Me}, 1 \mathrm{~b}$

$\mathrm{R}=\mathrm{OMe}, 1 \mathrm{c}$

$\mathrm{R}=\mathrm{Cl}, \mathbf{1 d}$

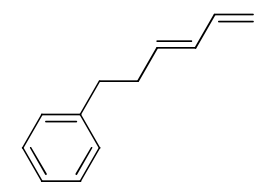

1j

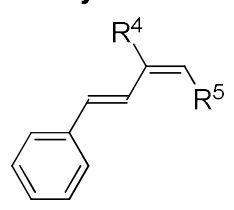

$\mathrm{R}^{4}=\mathrm{Me}, \quad \mathrm{R}^{5}=\mathrm{H}$

$\mathrm{R}^{4}=\mathrm{Me}, \quad \mathrm{R}^{5}=\mathrm{Me}, \quad 1 \mathrm{q}$

$\mathrm{R}^{4}=$ Me, $\quad \mathrm{R}^{5}=n$-butyl, $1 \mathrm{r}$

$\mathrm{R}^{4}=\mathrm{Me}, \quad \mathrm{R}^{5}=\mathrm{Ph}, \quad$ 1s

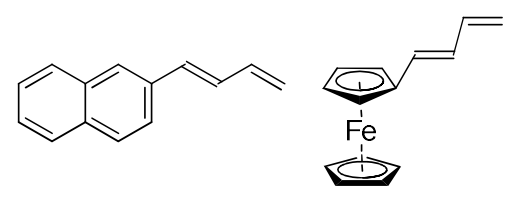

$1 f$
$1 \mathrm{~g}$

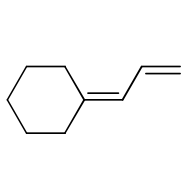

$1 \mathrm{~m}$

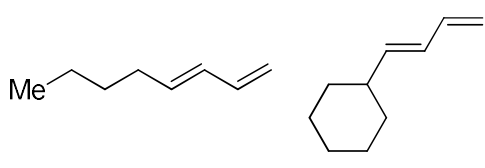

$1 \mathrm{~h}$

$1 \mathrm{i}$

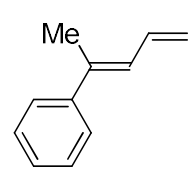

$1 \mathrm{k}$

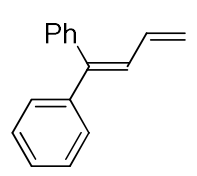

1 I

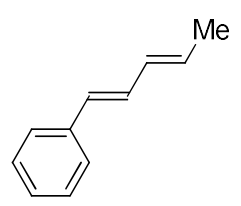

1n

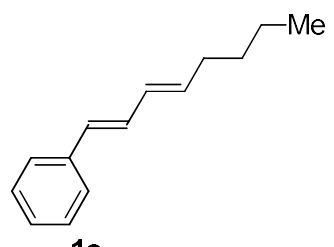

10<smiles>C=CC(=C)C</smiles><smiles>C=CC(=C)CCC=C(C)C</smiles><smiles>C1=CCCC=C1</smiles>

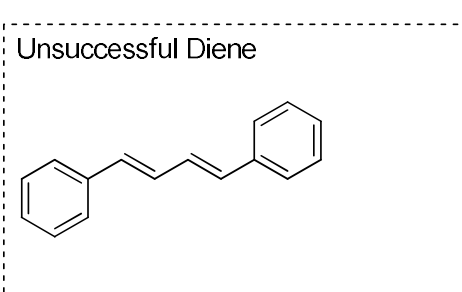

Successful Thiols<smiles>Sc1ccccc1</smiles><smiles>[Se]c1ccccc1</smiles>

$2 a$

2a-d<smiles>Sc1ccc(Cl)cc1</smiles><smiles>COc1ccc(S)cc1</smiles><smiles>Sc1cccc2ccccc12</smiles><smiles>SCc1ccccc1</smiles><smiles>SCc1ccco1</smiles>

$2 b$

2c

2d

$2 e$

2f<smiles>CC(C)CCS</smiles>

Unuccessful Thiols<smiles>SC(c1ccccc1)(c1ccccc1)c1ccccc1</smiles>

HS $Y_{N}^{N}=N^{N-H}$<smiles>COC(=O)C(CS)NC(C)=O</smiles> 


\subsection{General Procedure for the Reaction of 1,3-Dienes and Thiols (GP):}

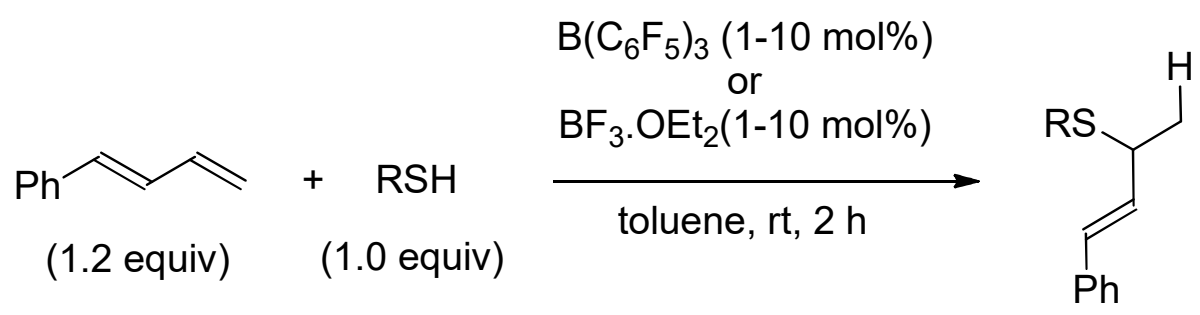

Procedure A : In an argon filled glove box, an oven-dried glass vial is charged with a magnetic stir bar, tris(pentafluorophenyl)borane $\left(B\left(\mathrm{C}_{6} \mathrm{~F}_{5}\right)_{3}\right)(1.0-10.0 \mathrm{~mol} \%)$ and toluene. Thiol 2a-2k (1.0 equiv) and diene $1 \mathbf{a}-1 \mathbf{v}$ (1.2 equiv) simultaneously added to the mixture. The vial has been taken out from the glove box and stirred for two hours at room temperature. The crude reaction mixture is used to measure the regiomeric ratio $(r r), E / Z$ ratio and diastereomeric ratio $(d r)$ by ${ }^{1} \mathrm{H}$ NMR. The analytically pure product is obtained by column chromatography on silica gel using petroleum ether and ethyl acetate as eluent.

Procedure B: $\quad$ In an argon filled glove box, an oven-dried glass vial is charged with a magnetic stir bar, thiol $2 \mathbf{a}-2 \mathbf{k}$ (1.0 equiv), diene $1 \mathbf{a}-1 \mathbf{v}$ (1.2 equiv) mixed in toluene, Boron trifluoride ethyl etherate $\left(\mathrm{BF}_{3} \cdot \mathrm{Et}_{2} \mathrm{O}\right)(1.0-10.0 \mathrm{~mol} \%)$ added to the mixture. The vial has been taken out from the glove box and stirred for two hours at room temperature. The crude reaction mixture is used to measure the regiomeric ratio $(r r), E / Z$ ratio and diastereomeric ratio $(d r)$ by ${ }^{1} \mathrm{H}$ NMR. The analytically pure product is obtained by column chromatography on silica gel using petroleum ether and ethyl acetate as eluent. 


\subsection{Experimental Details for the Synthesized Compounds}

\section{1. (E)-phenyl(4-phenylbut-3-en-2-yl)sulfane (3)}

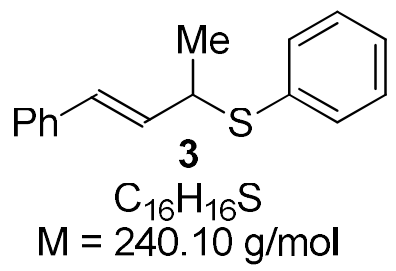

According to GP-A, using 1a (91.0 mg, $0.69 \mathrm{mmol}, 1.2$ equiv), 2a (64.0 mg, $0.58 \mathrm{mmol}, 1.0$ equiv), and tris(pentafluorophenyl)borane $\left(B\left(\mathrm{C}_{6} \mathrm{~F}_{5}\right)_{3}\right)(3.0 \mathrm{mg}, 5.8 \mu \mathrm{mol}, 1.0 \mathrm{~mol} \%)$. Purification by column chromatography on silica gel using petroleum ether as eluent. The product 3 (137.0 mg, 98\%) was obtained as colorless liquid. Reaction was also performed by GP-B, using 1a (141.0 mg, $1.08 \mathrm{mmol}, 1.2$ equiv), 2a (99.0 mg, $0.90 \mathrm{mmol}, 1.0$ equiv) and Boron trifluoride ethyl etherate $\left(\mathrm{BF}_{3} \cdot \mathrm{Et}_{2} \mathrm{O}\right)(1.3 \mathrm{mg}, 9.0 \mu \mathrm{mol}, 1.0 \mathrm{~mol} \%)$. Purification by column chromatography on silica gel using petroleum ether as eluent. The product 3 (190.0 mg, 88\%) was obtained as colorless liquid. MS-MS (ESI): for $\mathrm{C}_{16} \mathrm{H}_{16} \mathrm{~S}^{+}\left[(\mathrm{M}+\mathrm{H})^{+}\right]$: calculated 241.11, found 241.08 along with the other fragments $163.05,130.07,105.07,91.05 .{ }^{1} \mathbf{H}$ NMR $\left(400 \mathrm{MHz}, \mathrm{CDCl}_{3}\right) \delta$ 7.46-7.43 (m, 2H), 7.30-7.28 (m, 5H), , 7.27-7.22 (m, 3H), 6.26-6.15 $(\mathrm{m}, 2 \mathrm{H}), 3.96-3.89(\mathrm{~m}, 1 \mathrm{H}), 1.50(\mathrm{~d}, \mathrm{~J}=6.9 \mathrm{~Hz}, 3 \mathrm{H}) .{ }^{13} \mathrm{C}$ NMR $\left(101 \mathrm{MHz}, \mathrm{CDCl}_{3}\right) \delta$ 136.9, 134.6, 133.4, 131.7, 130.0, 128.8, 128.6, 127.5, 127.4, 126.4, 46.6, 20.7.

\section{2 (E)-(4-chlorophenyl)(4-phenylbut-3-en-2-yl)sulfane (4)}

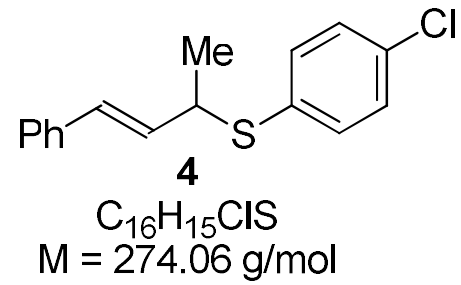

According to GP-A, using 1a (91.0 mg, $0.69 \mathrm{mmol}, 1.2$ equiv), 2b (84.0 mg, $0.58 \mathrm{mmol}, 1.0$ equiv), and tris(pentafluorophenyl)borane $\left(B\left(\mathrm{C}_{6} \mathrm{~F}_{5}\right)_{3}\right)(3.0 \mathrm{mg}, 5.8 \mu \mathrm{mol}, 1.0 \mathrm{~mol} \%)$. Purification by column chromatography on silica gel using petroleum ether as eluent. The product 4 (126.0, 79\%) was obtained as colorless liquid. Reaction was also performed by GP-B, using 1a (141.0 mg, $1.08 \mathrm{mmol}, 1.2$ equiv), 2b (130.0 mg, $0.90 \mathrm{mmol}, 1.0$ equiv) and Boron trifluoride ethyl etherate $\left(\mathrm{BF}_{3} \cdot \mathrm{Et}_{2} \mathrm{O}\right)(1.3 \mathrm{mg}, 9.0 \mu \mathrm{mol}, 1.0 \mathrm{~mol} \%)$. The product $4(190.0$ mg, $77 \%$ ) was obtained as colorless liquid. MS-MS (ESI): for $\mathrm{C}_{16} \mathrm{H}_{15} \mathrm{ClS}^{+}\left[(\mathrm{M}+\mathrm{H})^{+}\right]$: calculated 275.07, found 275.27 along with the other fragments $130.07,105.05,91.05 .{ }^{1} \mathrm{H}$ NMR (400 $\left.\mathrm{MHz}, \mathrm{CDCl}_{3}\right) \delta 7.36-7.33(\mathrm{~m}, 2 \mathrm{H}), 7.30-7.21(\mathrm{~m}, 7 \mathrm{H}), 6.22-6.10(\mathrm{~m}, 2 \mathrm{H}), 3.90-3.83(\mathrm{~m}, 1 \mathrm{H})$, 
$1.48(\mathrm{~d}, J=7.2 \mathrm{~Hz}, 3 \mathrm{H}) .{ }^{13} \mathrm{C}$ NMR $\left(101 \mathrm{MHz}, \mathrm{CDCl}_{3}\right) \delta 136.7,134.9,133.7,133.1,131.4$, $130.3,129.0,128.7,127.7,126.4,47.0,20.6$.

\section{$6.3 \quad(E)-(4-m e t h o x y p h e n y l)(4-p h e n y l b u t-3-e n-2-y l)$ sulfane (5)}

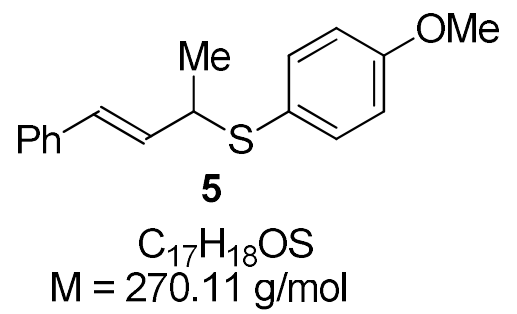

According to GP-A, using 1a (19.0 mg, $0.14 \mathrm{mmol}, 1.2$ equiv), 2c (167.0 mg, $0.12 \mathrm{mmol}, 1.0$ equiv), and tris(pentafluorophenyl)borane $\left(B\left(\mathrm{C}_{6} \mathrm{~F}_{5}\right)_{3}\right)(3.0 \mathrm{mg}, 6.0 \mu \mathrm{mol}, 5.0 \mathrm{~mol} \%)$. Purification by column chromatography on silica gel using petroleum ether as eluent. The product 5 (32.0 mg, 98\%) was obtained as colorless liquid. Reaction was also performed by GP-B, using 1a (141.0 mg, $1.08 \mathrm{mmol}, 1.2$ equiv), 2c (126.0 mg, $0.90 \mathrm{mmol}, 1.0$ equiv) and Boron trifluoride ethyl etherate $\left(\mathrm{BF}_{3} \cdot \mathrm{Et}_{2} \mathrm{O}\right)(1.3 \mathrm{mg}, 9.0 \mu \mathrm{mol}, 1.0 \mathrm{~mol} \%)$. Purification by column chromatography on silica gel using petroleum ether as eluent. The product $\mathbf{5}$ (51.0 mg, $21 \%$ ) was obtained as colorless liquid. HRMS (ESI): for $\mathrm{C}_{17} \mathrm{H}_{18} \mathrm{OS}^{+}\left[(\mathrm{M}+\mathrm{H})^{+}\right]$: calculated 271.1157, found 271.1147. ${ }^{1} \mathrm{H}$ NMR (400 MHz, $\left.\mathrm{CDCl}_{3}\right) \delta$ 7.39-7.36 (m, 2H), 7.29-7.26 (m, 4H), 7.23-7.21 (m, 1H), 6.82-6.80 (m, 2H), 6.18-6.08 (m, 2H), $3.78(\mathrm{~s}, 3 \mathrm{H}), 3.77-3.69(\mathrm{~m}$, $1 \mathrm{H}), 1.44(\mathrm{~d}, J=6.9 \mathrm{~Hz}, 3 \mathrm{H}) .{ }^{13} \mathrm{C}$ NMR $\left(101 \mathrm{MHz}, \mathrm{CDCl}_{3}\right) \delta 159.8,137.1,136.8,131.9,129.8$, $128.6,127.5,126.4,124.6,114.3,55.4,47.7,20.5$.

\section{4 (E)-naphthalen-1-yl(4-phenylbut-3-en-2-yl)sulfane (6)}

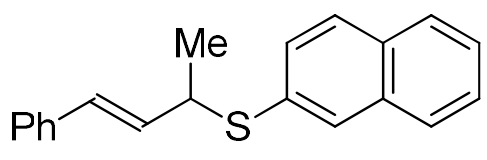

6

$$
\begin{gathered}
\mathrm{C}_{20} \mathrm{H}_{18} \mathrm{~S} \\
M=290.11 \mathrm{~g} / \mathrm{mol}
\end{gathered}
$$

According to GP-A, using 1a (91.0 mg, $0.69 \mathrm{mmol}, 1.2$ equiv), 2d (91.0 mg, $0.58 \mathrm{mmol}, 1.0$ equiv), and tris(pentafluorophenyl)borane $\left(B\left(\mathrm{C}_{6} \mathrm{~F}_{5}\right)_{3}\right)(3.0 \mathrm{mg}, 5.8 \mu \mathrm{mol}, 1.0 \mathrm{~mol} \%)$. Purification by column chromatography on silica gel using petroleum ether as eluent. The product 6 (151.0 mg, 90\%) was obtained as yellow solid. Reaction was also performed by GP-B, using 1a (141.0 mg, $1.08 \mathrm{mmol}, 1.2$ equiv), 2d (144.0 mg, $0.90 \mathrm{mmol}, 1.0$ equiv) and Boron trifluoride ethyl etherate $\left(\mathrm{BF}_{3} \cdot \mathrm{Et}_{2} \mathrm{O}\right)(1.3 \mathrm{mg}, 9.0 \mu \mathrm{mol}, 1.0 \mathrm{~mol} \%)$. Purification by column chromatography on silica gel using petroleum ether as eluent. The product 6 (259.0 
mg, 99\%) was obtained as yellow solid. M.P- $60-61{ }^{\circ} \mathrm{C}$. MS-MS (ESI): for $\mathrm{C}_{20} \mathrm{H}_{18} \mathrm{~S}^{+}\left[(\mathrm{M}+\mathrm{H})^{+}\right]$: calculated 291.12, found 291.08 along with the other fragments $212.06,187.05,130.07$, 129.07, 91.05. ${ }^{1} \mathrm{H}$ NMR (400 MHz, $\left.\mathrm{CDCl}_{3}\right) \delta 7.93(\mathrm{~s}, 1 \mathrm{H}), 7.81-7.74(\mathrm{~m}, 3 \mathrm{H}), 7.53-7.44(\mathrm{~m}$, $3 \mathrm{H}), 7.28-7.19(\mathrm{~m}, 5 \mathrm{H}), 6.32-6.21(\mathrm{~m}, 2 \mathrm{H}), 4.08-4.01(\mathrm{~m}, 1 \mathrm{H}), 1.55(\mathrm{~d}, J=8.9 \mathrm{~Hz}, 3 \mathrm{H}) .{ }^{13} \mathrm{C}$ NMR $\left(101 \mathrm{MHz}, \mathrm{CDCl}_{3}\right) \delta 136.9,133.7,132.5,132.1,132.0,131.7,130.6,130.2,128.6$, $128.3,127.8,127.6(2 \mathrm{C}), 126.5,126.4,126.2,46.5,20.8$.

\section{$6.5 \quad(E)$-benzyl(4-phenylbut-3-en-2-yl)sulfane (7)}

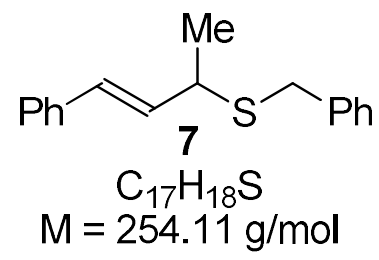

According to GP-A, using 1a (19.0 mg, $0.14 \mathrm{mmol}, 1.2$ equiv), $2 \mathrm{e}$ (15.0 mg, $0.12 \mathrm{mmol}, 1.0$ equiv), and tris(pentafluorophenyl)borane $\left(B\left(\mathrm{C}_{6} \mathrm{~F}_{5}\right)_{3}\right)(6.0 \mathrm{mg}, 12.0 \mu \mathrm{mol}, 10.0 \mathrm{~mol} \%)$. Purification by column chromatography on silica gel using petroleum ether as eluent. The product 7 (21.0 mg, 68\%) was obtained as colorless liquid. Reaction was also performed by GP-B, using 1a (141.0 mg, $1.08 \mathrm{mmol}, 1.2$ equiv), 2e (112.0 mg, $0.90 \mathrm{mmol}, 1.0$ equiv) and Boron trifluoride ethyl etherate $\left(\mathrm{BF}_{3} \cdot \mathrm{Et}_{2} \mathrm{O}\right)(1.3 \mathrm{mg}, 9.0 \mu \mathrm{mol}, 1.0 \mathrm{~mol} \%)$. Purification by column chromatography on silica gel using petroleum ether as eluent. The product 7 (224.0 mg, 98\%) was obtained as colorless liquid. MS-MS (ESI): for $\mathrm{C}_{17} \mathrm{H}_{18} \mathrm{~S}^{+}\left[(\mathrm{M}+\mathrm{H})^{+}\right]$: calculated 255.12, found 255.09 along with the other fragments $241.10,179.08,131.08,91.05$. ${ }^{1} \mathbf{H}$ NMR $\left(400 \mathrm{MHz}, \mathrm{CDCl}_{3}\right) \delta 7.42(\mathrm{~d}, J=7.6 \mathrm{~Hz}, 2 \mathrm{H}), 7.38-7.32(\mathrm{~m}, 6 \mathrm{H}), 7.29-7.24(\mathrm{~m}, 2 \mathrm{H}), 6.36(\mathrm{~d}$, $J=15.6 \mathrm{~Hz}, 1 \mathrm{H}), 6.12(\mathrm{dd}, J=15.6,8.9 \mathrm{~Hz}, 1 \mathrm{H}), 3.71(\mathrm{~s}, 2 \mathrm{H}), 3.47-3.39(\mathrm{~m}, 1 \mathrm{H}), 1.41(\mathrm{~d}, J=$ $6.8 \mathrm{~Hz}, 3 \mathrm{H}) .{ }^{13} \mathrm{C}$ NMR $\left(101 \mathrm{MHz}, \mathrm{CDCl}_{3}\right) \delta 138.7,136.8,132.3,130.0,129.0,128.7,128.6$, 127.6, 127.0, 126.4, 42.2, 35.4, 20.6 . 


\section{$6.6 \quad(E)-2-(((4-p h e n y l b u t-3-e n-2-y l)$ thio)methyl)furan (8)}

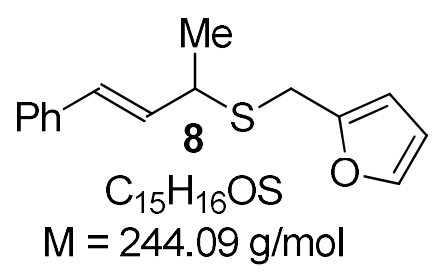

According to GP-A, using 1a (19.0 mg, $0.14 \mathrm{mmol}, 1.2$ equiv), $2 \mathrm{f}$ (14.0 mg, $0.12 \mathrm{mmol}, 1.0$ equiv), and tris(pentafluorophenyl)borane $\left(\mathrm{B}_{(}\left(\mathrm{C}_{6} \mathrm{~F}_{5}\right)_{3}\right)(3.0 \mathrm{mg}, 6.0 \mu \mathrm{mol}, 5.0 \mathrm{~mol} \%)$. Purification by column chromatography on silica gel using petroleum ether as eluent. The product 8 (14.0 mg, 48\%) was obtained as yellow liquid. Reaction was also performed by GP-B, using 1a (141.0 mg, $1.08 \mathrm{mmol}, 1.2$ equiv), 2 f (103.0 mg, $0.90 \mathrm{mmol}, 1.0$ equiv) and Boron trifluoride ethyl etherate $\left(\mathrm{BF}_{3} \cdot \mathrm{Et}_{2} \mathrm{O}\right)(1.3 \mathrm{mg}, 9.0 \mu \mathrm{mol}, 1.0 \mathrm{~mol} \%)$. Purification by column chromatography on silica gel using petroleum ether as eluent. The product 8 (110.0 mg, $50 \%$ ) was obtained as yellow liquid. MS-MS (ESI): for $\mathrm{C}_{15} \mathrm{H}_{16} \mathrm{OS}^{+}\left[(\mathrm{M}+\mathrm{H})^{+}\right]$: calculated 245.10, found 245.09 along with the other fragments $141.03,131.08,91.05 .{ }^{1} \mathbf{H}$ NMR (400 $\left.\mathrm{MHz}, \mathrm{CDCl}_{3}\right) \delta 7.42-7.37(\mathrm{~m}, 3 \mathrm{H}), 7.33(\mathrm{t}, J=7.5 \mathrm{~Hz}, 2 \mathrm{H}), 7.27-7.23(\mathrm{~m}, 1 \mathrm{H}), 6.41(\mathrm{~d}, J=$ $15.7 \mathrm{~Hz}, 1 \mathrm{H}), 6.31(\mathrm{dd}, J=3.2,1.8 \mathrm{~Hz}, 1 \mathrm{H}), 6.16(\mathrm{~d}, J=3.3 \mathrm{~Hz}, 1 \mathrm{H}), 6.06$ (dd, $J=15.6,9.2$ $\mathrm{Hz}, 1 \mathrm{H}), 3.73-3.62(\mathrm{~m}, 2 \mathrm{H}), 3.57-3.50(\mathrm{~m}, 1 \mathrm{H}), 1.41(\mathrm{~d}, J=6.9 \mathrm{~Hz}, 3 \mathrm{H}) .{ }^{13} \mathrm{C}$ NMR $(101 \mathrm{MHz}$, $\left.\mathrm{CDCl}_{3}\right) \delta 152.3,142.1,136.7,131.8,130.3,128.7,127.7,126.5,110.5,107.3,42.5,27.5$, 20.4 .

\section{$6.7 \quad(E)$-isopentyl(4-phenylbut-3-en-2-yl)sulfane (9)}

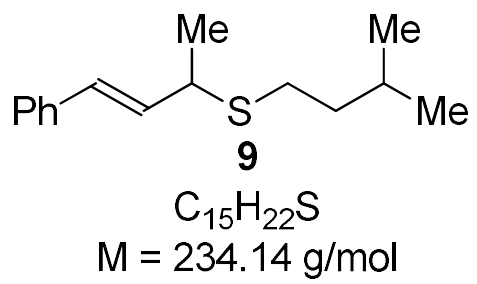

According to GP-A, using 1a (19.0 mg, $0.14 \mathrm{mmol}, 1.2$ equiv), $\mathbf{2 g}$ (13.0 mg, $0.12 \mathrm{mmol}, 1.0$ equiv), and tris(pentafluorophenyl)borane $\left(B\left(\mathrm{C}_{6} \mathrm{~F}_{5}\right)_{3}\right)(6.0 \mathrm{mg}, 12.0 \mu \mathrm{mol}, 10.0 \mathrm{~mol} \%)$. Purification by column chromatography on silica gel using petroleum ether as eluent. The product 9 (25.0 $\mathrm{mg}, 88 \%)$ obtained as colorless liquid. Reaction was also performed by GP. B, using 1a (141.0 mg, $1.08 \mathrm{mmol}, 1.2$ equiv), $2 \mathrm{~g}$ (94.0 mg, $0.90 \mathrm{mmol}, 1.0$ equiv) and Boron trifluoride ethyl etherate $\left(\mathrm{BF}_{3} \cdot \mathrm{Et}_{2} \mathrm{O}\right)(1.3 \mathrm{mg}, 9.0 \mu \mathrm{mol}, 1.0 \mathrm{~mol} \%)$. Purification by column chromatography on silica gel using petroleum ether as eluent. The product 9 (207.0, 98\%) was obtained as colorless liquid. MS-MS (ESI): for $\mathrm{C}_{15} \mathrm{H}_{22} \mathrm{~S}^{+}\left[(\mathrm{M}+\mathrm{H})^{+}\right]$: calculated 235.15, found 235.15 along with the other fragments 204.06, 179.07, 130.04, 129.04, 91.05. ${ }^{1} \mathbf{H}$ NMR 
(400 MHz, $\left.\mathrm{CDCl}_{3}\right)$ ठ 7.39-7.37 (m, 2H), 7.33-7.30 (m, 2H), 7.25-7.21 (m, 1H), $6.35(\mathrm{~d}, \mathrm{~J}=$ $15.6 \mathrm{~Hz}, 1 \mathrm{H}), 6.05(\mathrm{dd}, J=15.6,9.1 \mathrm{~Hz}, 1 \mathrm{H}), 3.54-3.47(\mathrm{~m}, 1 \mathrm{H}), 2.54-2.39(\mathrm{~m}, 2 \mathrm{H}), 1.67-$ $1.61(\mathrm{~m}, 1 \mathrm{H}), 1.46(\mathrm{q}, J=7.5 \mathrm{~Hz}, 2 \mathrm{H}), 1.41(\mathrm{~d}, J=6.8 \mathrm{~Hz}, 3 \mathrm{H}), 0.87$ (dd, $J=6.7,4.2 \mathrm{~Hz}$, $6 \mathrm{H}) .{ }^{13} \mathrm{C}$ NMR $\left(101 \mathrm{MHz}, \mathrm{CDCl}_{3}\right) \delta 136.9,132.7,129.4,128.7,127.5,126.4,42.6,38.9,28.9$, $27.7,22.4,20.8$.

\section{8 (E)-cyclohexyl(4-phenylbut-3-en-2-yl)sulfane (10)}

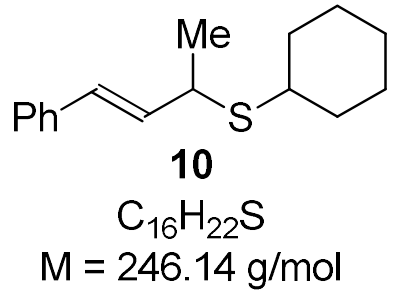

According to GP-A, using 1a (19.0 mg, $0.14 \mathrm{mmol}, 1.2$ equiv), $2 \mathrm{~h}$ (14.0 mg, $0.12 \mathrm{mmol}, 1.0$ equiv), and tris(pentafluorophenyl)borane $\left(B\left(\mathrm{C}_{6} \mathrm{~F}_{5}\right)_{3}\right)(6.0 \mathrm{mg}, 12.0 \mu \mathrm{mol}, 10.0 \mathrm{~mol} \%)$. Purification by column chromatography on silica gel using petroleum ether as eluent. The product 10 (26.0 mg, 89\%) was obtained as colorless liquid. Reaction was also performed by GP-B, using 1a (141.0 mg, $1.08 \mathrm{mmol}, 1.2$ equiv), 2h (105.0 mg, $0.90 \mathrm{mmol}, 1.0$ equiv) and Boron trifluoride ethyl etherate $\left(\mathrm{BF}_{3} \cdot \mathrm{Et}_{2} \mathrm{O}\right)(1.3 \mathrm{mg}, 9.0 \mu \mathrm{mol}, 1.0 \mathrm{~mol} \%)$. Purification by column chromatography on silica gel using petroleum ether as eluent. The product 10 (219.0 mg, 99\%) was obtained as colorless liquid. MS-MS (ESI): for $\mathrm{C}_{16} \mathrm{H}_{22} \mathrm{~S}^{+}\left[(\mathrm{M}+\mathrm{H})^{+}\right]$: calculated 247.15 , found 247.09 along with the other fragments $218.11,143.09,131.08,129.07,91.05$. ${ }^{1} \mathrm{H}$ NMR $\left(400 \mathrm{MHz}, \mathrm{CDCl}_{3}\right) \delta$ 7.41-7.36 (m, 2H), 7.34-1.30 (m, 2H), 7.25-7.21 (m, 1H), 6.36 (d, $J=15.7 \mathrm{~Hz}, 1 \mathrm{H}), 6.08(\mathrm{dd}, J=15.6,9.1 \mathrm{~Hz}, 1 \mathrm{H}), 3.66-3.59(\mathrm{~m}, 1 \mathrm{H}), 2.65-2.58(\mathrm{~m}, 1 \mathrm{H})$, 2.04-1.99 (m, 1H), 1.88-1.83 (m, 1H), 1.78-1.68 (m, 2H), $1.39(\mathrm{~d}, J=6.9 \mathrm{~Hz}, 3 \mathrm{H}), 1.37-$ $1.21(\mathrm{~m}, 6 \mathrm{H}) .{ }^{13} \mathrm{C}$ NMR $\left(101 \mathrm{MHz}, \mathrm{CDCl}_{3}\right) \delta 137.0,133.3,128.9,128.7,127.5,126.4,42.5$, $41.1,34.3,33.6,26.3,26.0,21.1$.

\section{$6.9 \quad(E)$-benzyl 3-((4-phenylbut-3-en-2-yl)thio)propanoate (11)}

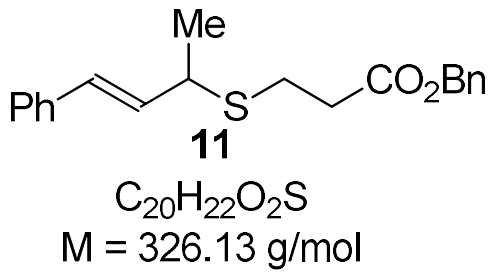

According to GP-A, at $80{ }^{\circ} \mathrm{C}$ temperature, using $1 \mathrm{a}$ (19.0 mg, $0.14 \mathrm{mmol}, 1.2$ equiv), $2 \mathbf{i}(24.0$ $\mathrm{mg}, 0.12 \mathrm{mmol}, 1.0$ equiv), and tris(pentafluorophenyl)borane $\left(B\left(\mathrm{C}_{6} \mathrm{~F}_{5}\right)_{3}\right)(3.0 \mathrm{mg}, 6.0 \mu \mathrm{mol}$, $5.0 \mathrm{~mol} \%$ ). Purification by column chromatography on silica gel using petroleum ether/ethyl acetate (20:1). The product 11 (37.0 mg, 94\%) was obtained as colorless liquid. Reaction was also performed by GP-B, at $80{ }^{\circ} \mathrm{C}$ temperature using $1 \mathrm{a}(14.0 \mathrm{mg}, 0.11 \mathrm{mmol}, 1.2$ 
equiv), $2 \mathbf{i}$ (18.0 mg, $0.09 \mathrm{mmol}, 1.0$ equiv) and Boron trifluoride ethyl etherate $\left(\mathrm{BF}_{3} \cdot \mathrm{Et}_{2} \mathrm{O}\right)$ (1.3 $\mathrm{mg}, 9.0 \mu \mathrm{mol}, 10.0 \mathrm{~mol} \%$ ). Purification by column chromatography on silica gel using petroleum ether/ethyl acetate (20:1). The product $11(22.0 \mathrm{mg}, 76 \%)$ was obtained as colorless liquid. HRMS (ESI): for $\mathrm{C}_{20} \mathrm{H}_{22} \mathrm{O}_{2} \mathrm{~S}^{+}\left[(\mathrm{M}+\mathrm{Na})^{+}\right]$: calculated 349.1238, found 349.1245. ' $\mathrm{H}$ NMR (400 MHz, $\left.\mathrm{CDCl}_{3}\right) \delta 7.38-7.29(\mathrm{~m}, 9 \mathrm{H}), 7.25-7.23(\mathrm{~m}, 1 \mathrm{H}), 6.38(\mathrm{~d}, \mathrm{~J}=$ $15.5 \mathrm{~Hz}, 1 \mathrm{H}), 6.04$ (dd, J = 15.7, $9.1 \mathrm{~Hz}, 1 \mathrm{H}), 5.13$ (s, 2H), 3.57-3.49 (m, 1H), 2.79-2.74 (m, 2H), 2.66-2.62 (m, 2H), $1.40(\mathrm{~d}, J=6.8 \mathrm{~Hz}, 3 \mathrm{H}) .{ }^{13} \mathrm{C}$ NMR $\left(101 \mathrm{MHz}, \mathrm{CDCl}_{3}\right) \delta 171.9,136.7$, 135.9, 132.2, 129.8, 128.7(2C), 128.4(2C), 127.7, 126.5(2C), 66.6, 43.0, 35.1, 25.9, 20.7.

\subsection{0 (E)-(4-phenylbut-3-en-2-yl)(1-phenylpropan-2-yl)sulfane (12)}

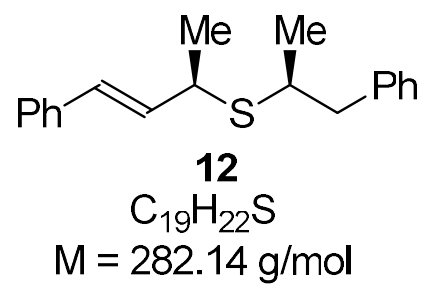

According to GP-A, using 1a (91.0 mg, $0.69 \mathrm{mmol}, 1.2$ equiv), 2j (83.0 mg, $0.58 \mathrm{mmol}, 1.0$ equiv), and tris(pentafluorophenyl)borane $\left(B\left(\mathrm{C}_{6} \mathrm{~F}_{5}\right)_{3}\right) \quad(3.0 \mathrm{mg}, 5.8 \mu \mathrm{mol}, 1.0 \mathrm{~mol} \%)$. Purification by column chromatography on silica gel using petroleum ether as eluent. The product $12+12^{\prime}(139.0 \mathrm{mg}, 85 \%, d r=1.3: 1$ from crude) was obtained as colorless liquid. Reaction was also performed by GP-B, using $1 \mathrm{a}$ (141.0 mg, $1.08 \mathrm{mmol}, 1.2$ equiv), 2j (137.0 $\mathrm{mg}, 0.90 \mathrm{mmol}, 1.0$ equiv) and Boron trifluoride ethyl etherate $\left(\mathrm{BF}_{3} \cdot \mathrm{Et}_{2} \mathrm{O}\right)(1.3 \mathrm{mg}, 9.0 \mu \mathrm{mol}$, $1.0 \mathrm{~mol} \%)$. Purification by column chromatography on silica gel using petroleum ether as eluent. The product $12+12 '(203.0 \mathrm{mg}, 80 \%, d r=1.6: 1$ from crude) obtained as colorless liquid. ${ }^{1} \mathrm{H}$ NMR $\left(400 \mathrm{MHz}, \mathrm{CDCl}_{3}\right) \delta 7.41-7.39(\mathrm{~m}, 2 \mathrm{H}), 7.34(\mathrm{t}, J=7.6 \mathrm{~Hz}, 2 \mathrm{H}), 7.29-7.21$ $(\mathrm{m}, 4 \mathrm{H}), 7.18-7.15(\mathrm{~m}, 2 \mathrm{H}), 6.34(\mathrm{~d}, J=15.8 \mathrm{~Hz}, 1 \mathrm{H}), 6.07$ (dd, J=15.7, 9.3 Hz, 1H), 3.52$3.44(\mathrm{~m}, 1 \mathrm{H}), 3.08(\mathrm{dd}, J=13.3,5.5 \mathrm{~Hz}, 1 \mathrm{H}), 2.99-2.90(\mathrm{~m}, 1 \mathrm{H}), 2.65(\mathrm{dd}, J=13.2,8.7 \mathrm{~Hz}$, $1 \mathrm{H}), 1.36(\mathrm{~d}, J=6.8 \mathrm{~Hz}, 3 \mathrm{H}), 1.16(\mathrm{~d}, J=6.9 \mathrm{~Hz}, 3 \mathrm{H}) .{ }^{13} \mathrm{C}$ NMR $\left(101 \mathrm{MHz}, \mathrm{CDCl}_{3}\right) \delta 139.8$, 136.8, 133.1, 129.5, 129.4, 128.8, 128.4, 127.6, 126.4(2C), 44.5, 42.1, 40.3, 20.9, 20.6.

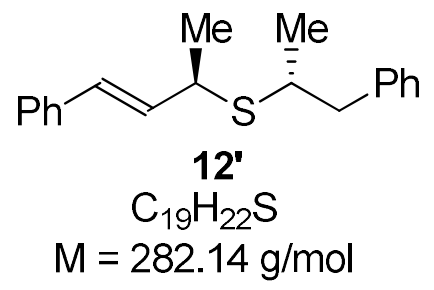


${ }^{1} \mathrm{H}$ NMR $\left(400 \mathrm{MHz}, \mathrm{CDCl}_{3}\right) \delta$ 7.38-7.31 (m, 4H), 7.26-7.17 (m, 4H),7.13 (d, J = 8.2 Hz, 2H), $6.37(\mathrm{~d}, J=15.7 \mathrm{~Hz}, 1 \mathrm{H}), 6.04(\mathrm{dd}, J=15.7,9.2 \mathrm{~Hz}, 1 \mathrm{H}), 3.61-3.56(\mathrm{~m}, 1 \mathrm{H}), 2.99-2.90(\mathrm{~m}$, 2H), 2.70-2.62 (m, 1H), $1.40(\mathrm{~d}, J=6.8 \mathrm{~Hz}, 3 \mathrm{H}), 1.27(\mathrm{~d}, J=6.6 \mathrm{~Hz}, 3 \mathrm{H}) .{ }^{13} \mathrm{C}$ NMR $(101 \mathrm{MHz}$, $\left.\mathrm{CDCl}_{3}\right) \delta 139.6,136.9,133.1,129.4,129.2,128.7,128.3,127.6,126.5,126.4,43.6,42.0$, 40.5, 21.4, 21.0 .

\subsection{1 (E)-3-((4-phenylbut-3-en-2-yl)thio)propane-1-thiol and ((E)-4-phenylbut-3-en-2- yl)(3-(((E)-5-phenylpent-4-en-2-yl)thio)propyl)sulfane $\left(13+13^{\prime}\right)$}

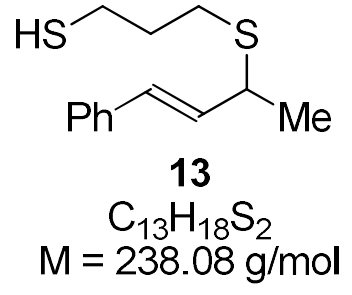

According to GP-A, using 1a (91.0 mg, 0.69 mmol, 1.2 equiv), 2k (63.0 mg, 0.58 mmol, 1.0 equiv), and tris(pentafluorophenyl)borane $\left(\mathrm{B}_{(}\left(\mathrm{C}_{6} \mathrm{~F}_{5}\right)_{3}\right)(3.0 \mathrm{mg}, 5.8 \mu \mathrm{mol}, 1.0 \mathrm{~mol} \%)$. Purification by column chromatography on silica gel using petroleum ether as eluent. The product $13(82.0 \mathrm{mg}, 59 \%)$ and 13' $^{\prime}(42.0 \mathrm{mg}, 19 \%)$, are obtained as colorless liquids. Reaction was also performed by GP-B, using 1a (141.0 mg, $1.08 \mathrm{mmol}, 1.2$ equiv), 2k (97.0 $\mathrm{mg}, 0.90 \mathrm{mmol}, 1.0$ equiv) and Boron trifluoride ethyl etherate $\left(\mathrm{BF}_{3} \cdot \mathrm{Et}_{2} \mathrm{O}\right)(1.3 \mathrm{mg}, 9.0 \mu \mathrm{mol}$, $1.0 \mathrm{~mol} \%$ ). Purification by column chromatography on silica gel using petroleum ether as eluent. The product $13(120.0 \mathrm{mg}, 56 \%)$ and 13' $^{\prime}(103.0 \mathrm{mg}, 30 \%)$ are obtained as colorless liquids. MS-MS (ESI): for $\mathrm{C}_{13} \mathrm{H}_{18} \mathrm{~S}_{2}{ }^{+}\left[(\mathrm{M}+\mathrm{H})^{+}\right]$: calculated 239.09, found 239.08 along with the other fragments 130.07, 129.07, 91.05. ${ }^{1} \mathrm{H}$ NMR (400 MHz, $\left.\mathrm{CDCl}_{3}\right) \delta 7.39-7.37(\mathrm{~m}, 2 \mathrm{H})$, 7.34-7.30 (m, 2H), 7.26-7.21 (m, 1H), $6.37(\mathrm{~d}, J=15.7 \mathrm{~Hz}, 1 \mathrm{H}), 6.04(\mathrm{dd}, J=15.8,9.0 \mathrm{~Hz}$, $1 \mathrm{H}), 3.54-3.45(\mathrm{~m}, 1 \mathrm{H}), 2.66-2.50(\mathrm{~m}, 4 \mathrm{H}), 1.90-1.83(\mathrm{~m}, 2 \mathrm{H}), 1.41(\mathrm{~d}, J=6.9 \mathrm{~Hz}, 3 \mathrm{H}), 1.33$ $(\mathrm{t}, J=8.1 \mathrm{~Hz}, 1 \mathrm{H}) .{ }^{13} \mathrm{C}$ NMR $\left(101 \mathrm{MHz}, \mathrm{CDCl}_{3}\right) \delta 136.7,132.4,129.7,128.8,127.7,126.4$, 42.8, 33.6, 29.2, 23.7, 20.8 .

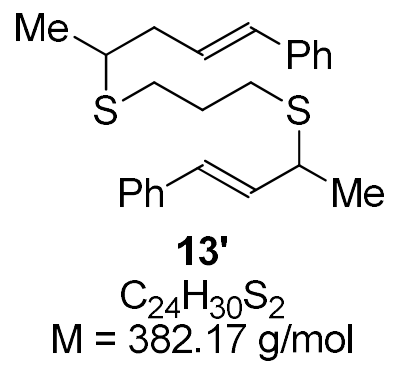

MS-MS (ESI): for $\mathrm{C}_{24} \mathrm{H}_{30} \mathrm{~S}_{2}{ }^{+}\left[(\mathrm{M}+\mathrm{H})^{+}\right]$: calculated 369.17 , found 369.39 along with the other fragments 131.08, 129.07, 117.07. ${ }^{1} \mathrm{H}$ NMR (400 MHz, $\left.\mathrm{CDCl}_{3}\right) \delta 7.37-7.35(\mathrm{~m}, 4 \mathrm{H}), 7.32$ $7.28(\mathrm{~m}, 4 \mathrm{H}), 7.25-7.21(\mathrm{~m}, 2 \mathrm{H}), 6.33$ (dd, $J=15.7,2.9 \mathrm{~Hz}, 2 \mathrm{H}), 6.02(\mathrm{dd}, J=15.7,9.1 \mathrm{~Hz}$, $2 \mathrm{H}), 3.50-3.43(\mathrm{~m}, 2 \mathrm{H}), 2.63-2.43(\mathrm{~m}, 4 \mathrm{H}), 1.87-1.80(\mathrm{~m}, 2 \mathrm{H}), 1.38(\mathrm{~d}, J=3.6 \mathrm{~Hz}, 3 \mathrm{H}), 1.37$ 
$(\mathrm{d}, J=3.6 \mathrm{~Hz}, 3 \mathrm{H}) .{ }^{13} \mathrm{C}$ NMR $\left(101 \mathrm{MHz}, \mathrm{CDCl}_{3}\right) \delta 136.8,132.5,129.6,128.7,127.6,126.5$, 42.7, 29.9, 29.8, 20.8 .

\subsection{2 (E)-phenyl(4-(p-tolyl)but-3-en-2-yl)sulfane (14)}<smiles>Cc1ccc(/C=C/C(C)c2ccccc2)cc1</smiles>

According to GP-A, using 1b (100.0 mg, 0.69 mmol, 1.2 equiv), 2a (64.0 mg, 0.58 mmol, 1.0 equiv), and tris(pentafluorophenyl)borane $\left(B\left(\mathrm{C}_{6} \mathrm{~F}_{5}\right)_{3}\right) \quad(3.0 \mathrm{mg}, 5.8 \mu \mathrm{mol}, 1.0 \mathrm{~mol} \%)$. Purification by column chromatography on silica gel using petroleum ether as eluent. The product 14 (115.0 mg, 78\%) was obtained as colorless liquid. Reaction was also performed by GP-B, using 1b (156.0 mg, $1.08 \mathrm{mmol}, 1.2$ equiv), 2a (99.0 mg, $0.90 \mathrm{mmol}, 1.0$ equiv) and Boron trifluoride ethyl etherate $\left(\mathrm{BF}_{3} \cdot \mathrm{Et}_{2} \mathrm{O}\right)(1.3 \mathrm{mg}, 9.0 \mu \mathrm{mol}, 1.0 \mathrm{~mol} \%)$. Purification by column chromatography on silica gel using petroleum ether as eluent. The product 14 (101.0, $44 \%$ ) was obtained as colorless liquid. MS-MS (ESI): for $\mathrm{C}_{17} \mathrm{H}_{18} \mathrm{~S}^{+}\left[(\mathrm{M}+\mathrm{H})^{+}\right]$: calculated 255.12, found 255.08 along with the other fragments, $146.07,145.07,144.06$. ${ }^{1} \mathbf{H}$ NMR (400 $\left.\mathrm{MHz}, \mathrm{CDCl}_{3}\right) \delta$ 7.46-7.43 (m, 2H), 7.31-7.24 (m, 3H), $7.20(\mathrm{~d}, J=8.1 \mathrm{~Hz}, 2 \mathrm{H}), 7.11$ (d, J = $7.8 \mathrm{~Hz}, 2 \mathrm{H}), 6.23-6.10(\mathrm{~m}, 2 \mathrm{H}), 3.96-3.88(\mathrm{~m}, 1 \mathrm{H}), 2.34(\mathrm{~s}, 3 \mathrm{H}), 1.50(\mathrm{~d}, J=6.8 \mathrm{~Hz}, 3 \mathrm{H}) .{ }^{13} \mathrm{C}$ NMR $\left(101 \mathrm{MHz}, \mathrm{CDCl}_{3}\right) \delta 137.3,134.7,134.2,133.4,130.7,130.0,129.3,128.8,127.4$, 126.3, 46.7, 21.3, 20.8. 


\subsection{3 (E)-(4-(4-methoxyphenyl)but-3-en-2-yl)(phenyl)sulfane (15)}<smiles>COc1ccc(/C=C/C(C)c2ccccc2)cc1</smiles>

According to GP-A, using 1c (112.0 mg, 0.69 mmol, 1.2 equiv), 2a (64.0 mg, 0.58 mmol, 1.0 equiv), and tris(pentafluorophenyl)borane $\left(\mathrm{B}_{(}\left(\mathrm{C}_{6} \mathrm{~F}_{5}\right)_{3}\right)(3.0 \mathrm{mg}, 5.8 \mu \mathrm{mol}, 1.0 \mathrm{~mol} \%)$. Purification by column chromatography on silica gel using petroleum ether as eluent. The product 15 (122.0 mg, 78\%) was obtained as colorless liquid. Reaction was also performed by GP-B, using 1c (173.0 mg, $1.08 \mathrm{mmol}, 1.2$ equiv), 2a (99.0 mg, $0.90 \mathrm{mmol}, 1.0$ equiv) and Boron trifluoride ethyl etherate $\left(\mathrm{BF}_{3} \cdot \mathrm{Et}_{2} \mathrm{O}\right)(1.3 \mathrm{mg}, 9.0 \mu \mathrm{mol}, 1.0 \mathrm{~mol} \%)$. Purification by column chromatography on silica gel using petroleum ether as eluent. The product 15 (47.0 mg, 20\%) was obtained as colorless loquid. MS-MS (ESI): for $\mathrm{C}_{17} \mathrm{H}_{18} \mathrm{OS}^{+}\left[(\mathrm{M}+\mathrm{H})^{+}\right]$: calculated 271.12, found 271.09 along with the other fragments 242.07, 161.09, 160.08, 145.06, 144.05. ${ }^{1} \mathrm{H}$ NMR $\left(400 \mathrm{MHz}, \mathrm{CDCl}_{3}\right) \delta 7.46-7.43(\mathrm{~m}, 2 \mathrm{H}), 7.31-7.22(\mathrm{~m}, 5 \mathrm{H}), 6.86-6.83(\mathrm{~m}$, $2 \mathrm{H}), 6.20(\mathrm{~d}, J=15.9 \mathrm{~Hz}, 1 \mathrm{H}), 6.05(\mathrm{dd}, J=15.6,8.2 \mathrm{~Hz}, 1 \mathrm{H}), 3.95-3.88(\mathrm{~m}, 1 \mathrm{H}), 3.82(\mathrm{~s}$, $3 \mathrm{H}), 1.50(\mathrm{~d}, J=6.9 \mathrm{~Hz}, 3 \mathrm{H}) .{ }^{13} \mathrm{C}$ NMR $\left(101 \mathrm{MHz}, \mathrm{CDCl}_{3}\right) \delta 159.2,134.8,133.4,129.7,129.5$, 128.8, 127.6 (2C), 127.4, 114.0, 55.4, 46.7, 20.9.

\subsection{4 (E)-(4-(4-chlorophenyl)but-3-en-2-yl)(phenyl)sulfane (16)}<smiles></smiles>

According to GP-A, using 1d (23.0 mg, $0.14 \mathrm{mmol}, 1.2$ equiv), 2a (13.0 mg, $0.12 \mathrm{mmol}, 1.0$ equiv), and tris(pentafluorophenyl)borane $\left(\mathrm{B}_{(}\left(\mathrm{C}_{6} \mathrm{~F}_{5}\right)_{3}\right)(6.0 \mathrm{mg}, 12.0 \mu \mathrm{mol}, 10.0 \mathrm{~mol} \%)$. Purification by column chromatography on silica gel using petroleum ether as eluent. The product 16 (23.0 mg, 70\%) was obtained as colorless liquid. Reaction was also performed by GP-B, using 1d (178.0 mg, $1.08 \mathrm{mmol}, 1.2$ equiv), 2a (99.0 mg, $0.90 \mathrm{mmol}, 1.0$ equiv) and Boron trifluoride ethyl etherate $\left(\mathrm{BF}_{3} \cdot \mathrm{Et}_{2} \mathrm{O}\right)(1.3 \mathrm{mg}, 9.0 \mu \mathrm{mol}, 1.0 \mathrm{~mol} \%)$. Purification by column chromatography on silica gel using petroleum ether as eluent. The product 16 (34.0 mg, 14\%) was obtained as colorless liquid. MS-MS (ESI): for $\mathrm{C}_{16} \mathrm{H}_{15} \mathrm{CIS}^{+}\left[(\mathrm{M}+\mathrm{H})^{+}\right]$: calculated 275.07, found 275.09 along with the other fragments, 165.03, 163.03, 135.07, 129.07, 128.06, 125.01. ${ }^{1} \mathrm{H}$ NMR $\left(400 \mathrm{MHz}, \mathrm{CDCl}_{3}\right) \delta 7.41-7.39(\mathrm{~m}, 2 \mathrm{H}), 7.28-7.16(\mathrm{~m}, 7 \mathrm{H}), 6.12-$ 
$6.11(\mathrm{~m}, 2 \mathrm{H}), 3.90-3.83(\mathrm{~m}, 1 \mathrm{H}), 1.47(\mathrm{~d}, J=6.8 \mathrm{~Hz}, 3 \mathrm{H}) .{ }^{13} \mathrm{C}$ NMR $\left(101 \mathrm{MHz}, \mathrm{CDCl}_{3}\right) \delta$ $135.4,134.4,133.6,133.1,132.3,128.8$ (2C), 128.8, 127.6, 127.6, 46.6, 20.6.

\subsection{5 (E)-2-(3-(phenylthio)but-1-en-1-yl)furan (17)}

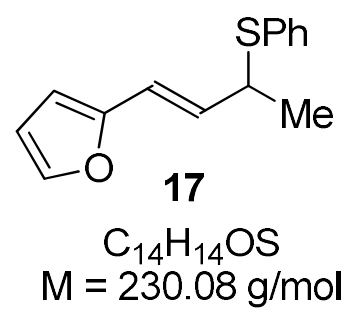

According to GP-A, using 1e (83.0 mg, $0.69 \mathrm{mmol}, 1.2$ equiv), 2a (64.0 mg, $0.58 \mathrm{mmol}, 1.0$ equiv), and tris(pentafluorophenyl)borane $\left(\mathrm{B}_{(}\left(\mathrm{C}_{6} \mathrm{~F}_{5}\right)_{3}\right)(3.0 \mathrm{mg}, 5.8 \mu \mathrm{mol}, 1.0 \mathrm{~mol} \%)$. Purification by column chromatography on silica gel using petroleum ether as eluent. The product 17 (131.0 mg, 98\%) was obtained as yellow liquid. Reaction was also performed by GP-B, using 1e (130.0 mg, $1.08 \mathrm{mmol}, 1.2$ equiv), 2a (99.0 mg, $0.90 \mathrm{mmol}, 1.0$ equiv) and Boron trifluoride ethyl etherate $\left(\mathrm{BF}_{3} \cdot \mathrm{Et}_{2} \mathrm{O}\right)(1.3 \mathrm{mg}, 9.0 \mu \mathrm{mol}, 1.0 \mathrm{~mol} \%)$. The product 17 was not detected. MS-MS (ESI): for $\mathrm{C}_{14} \mathrm{H}_{14} \mathrm{OS}^{+}\left[(\mathrm{M}+\mathrm{H})^{+}\right]$: calculated 231.08, found 231.07 along with the other fragments $162.04,120.05,105.07,95.05 .{ }^{1} \mathrm{H}$ NMR $\left(400 \mathrm{MHz}, \mathrm{CDCl}_{3}\right) \delta 7.42-$ $7.39(\mathrm{~m}, 2 \mathrm{H}), 7.32-7.21(\mathrm{~m}, 4 \mathrm{H}), 6.33(\mathrm{dd}, J=3.3,1.8 \mathrm{~Hz}, 1 \mathrm{H}), 6.18-6.04(\mathrm{~m}, 3 \mathrm{H}), 3.90-3.83$ $(\mathrm{m}, 1 \mathrm{H}), 1.46(\mathrm{~d}, J=6.8 \mathrm{~Hz}, 3 \mathrm{H}) .{ }^{13} \mathrm{C}$ NMR $\left(101 \mathrm{MHz}, \mathrm{CDCl}_{3}\right) \delta 152.4,142.0,134.6,133.2$, 130.3, 128.9, 127.4, 118.5, 111.4, 107.8, 46.3, 20.7.

\subsection{6 (E)-(4-(naphthalen-2-yl)but-3-en-2-yl)(phenyl)sulfane (18)}<smiles>CC(C=Cc1ccc2ccccc2c1)c1ccccc1</smiles>

$$
M=290.11 \mathrm{~g} / \mathrm{mol}
$$

According to GP-A, using $1 \mathbf{1 f}(125.0 \mathrm{mg}, 0.69 \mathrm{mmol}, 1.2$ equiv), 2a (64.0 mg, $0.58 \mathrm{mmol}, 1.0$

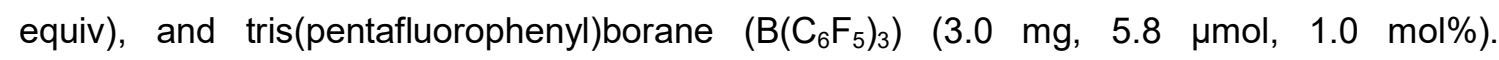
Purification by column chromatography on silica gel using petroleum ether as eluent. The product 18 (149.0, 88\%) was obtained as yellow solid. Reaction was also performed by GPB, using $1 \mathbf{f}$ (195.0 mg, $1.08 \mathrm{mmol}, 1.2$ equiv), 2a (99.0 mg, $0.90 \mathrm{mmol}, 1.0$ equiv) and Boron trifluoride ethyl etherate $\left(\mathrm{BF}_{3} \cdot \mathrm{Et}_{2} \mathrm{O}\right)(1.3 \mathrm{mg}, 9.0 \mu \mathrm{mol}, 1.0 \mathrm{~mol} \%)$. Purification by column chromatography on silica gel using petroleum ether as eluent. The product $18(151.0 \mathrm{mg}$, 
58\%) was obtained as yellow solid. M.P. $=40-41{ }^{\circ} \mathrm{C}$. MS-MS (ESI): for $\mathrm{C}_{20} \mathrm{H}_{18} \mathrm{~S}^{+}\left[(\mathrm{M}+\mathrm{H})^{+}\right]$: calculated 291.12, found 291.08 along with the other fragments $180.09,166.07,155.08$, 141.07. ${ }^{1} \mathbf{H}$ NMR (400 MHz, $\left.\mathrm{CDCl}_{3}\right) \delta 7.83-7.68(\mathrm{~m}, 3 \mathrm{H}), 7.51-7.39(\mathrm{~m}, 6 \mathrm{H}), 7.33-7.25(\mathrm{~m}$, $3 \mathrm{H}), 6.87(\mathrm{~d}, J=15.3 \mathrm{~Hz}, 1 \mathrm{H}), 6.14(\mathrm{dd}, J=15.5,8.6 \mathrm{~Hz}, 1 \mathrm{H}), 4.08-4.00(\mathrm{~m}, 1 \mathrm{H}), 1.58(\mathrm{~d}, J$ $=6.9 \mathrm{~Hz}, 3 \mathrm{H}) .{ }^{13} \mathrm{C}$ NMR $\left(101 \mathrm{MHz}, \mathrm{CDCl}_{3}\right) \delta 135.0,134.8,134.6,133.9,133.6,131.2,129.0$, $128.5,127.9,127.6,127.4,125.9,125.8,125.7,124.1,124.0,47.3,20.6$.

\subsection{7 (E)-(4-(ferrocene-2-yl)but-3-en-2-yl)(phenyl)sulfane (19) and (1-(ferrocene-2- yl)butane-1,3-diyl)bis(phenylsulfane) (19')}

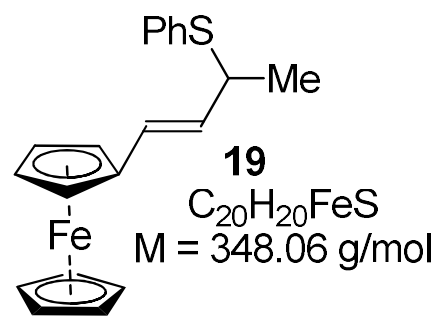

According to GP-A, using $1 \mathrm{~g}$ (139.0 mg, $0.69 \mathrm{mmol}, 1.2$ equiv), 2a (64.0 mg, $0.58 \mathrm{mmol}, 1.0$ equiv), and tris(pentafluorophenyl)borane $\left(B\left(\mathrm{C}_{6} \mathrm{~F}_{5}\right)_{3}\right)(3.0 \mathrm{mg}, 5.8 \mu \mathrm{mol}, 1.0 \mathrm{~mol} \%)$. Purification by column chromatography on silica gel using petroleum ether as eluent. The product 19 (89.0 mg, 44\%) and 19' (74.0 mg, 28\%) were obtained as yellow liquids. Reaction was also performed by GP-B, using $1 \mathrm{~g}$ (259.0 mg, $1.08 \mathrm{mmol}, 1.2$ equiv), 2a (99.0 mg, 0.90 mmol, 1.0 equiv) and Boron trifluoride ethyl etherate $\left(\mathrm{BF}_{3} \cdot \mathrm{Et}_{2} \mathrm{O}\right)(1.3 \mathrm{mg}, 9.0 \mu \mathrm{mol}, 1.0$ mol\%). Purification by column chromatography on silica gel using petroleum ether as eluent. The products $19(194.0 \mathrm{mg}, 62 \%)$ and 19' (21.0 mg, 5\%) were obtained as yellow liquids. HRMS (ESI): for $\mathrm{C}_{20} \mathrm{H}_{20} \mathrm{FeS}^{+}\left[\mathrm{M}^{+}\right]$: calculated 348.0635, found 348.0618. ${ }^{1} \mathrm{H}$ NMR (400 MHz, $\left.\mathrm{CDCl}_{3}\right) \delta 7.46(\mathrm{dd}, J=7.8,1.7 \mathrm{~Hz}, 2 \mathrm{H}), 7.32(\mathrm{dd}, J=8.3,6.9 \mathrm{~Hz}, 2 \mathrm{H}), 7.24-7.20(\mathrm{~m}, 1 \mathrm{H})$, $6.06(\mathrm{~d}, J=15.7 \mathrm{~Hz}, 1 \mathrm{H}), 5.76(\mathrm{dd}, J=15.6,8.4 \mathrm{~Hz}, 1 \mathrm{H}), 4.28-4.10(\mathrm{~m}, 5 \mathrm{H}), 3.95(\mathrm{~s}, 4 \mathrm{H})$, 3.93-3.85 (m, 1H), $1.42(\mathrm{~d}, J=6.8 \mathrm{~Hz}, 3 \mathrm{H}) .{ }^{13} \mathrm{C}$ NMR $\left(101 \mathrm{MHz}, \mathrm{CDCl}_{3}\right) \delta 135.2,132.5$, $129.4,128.9,127.7,127.2,82.7,69.2,68.8,68.6,67.4,66.1,46.3,21.0$.

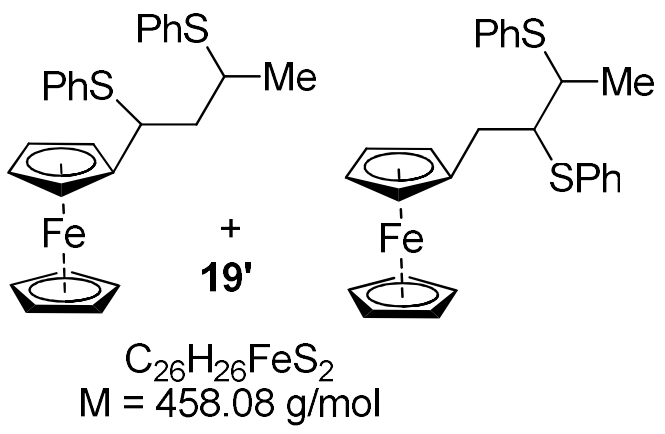


HRMS (ESI): for $\mathrm{C}_{26} \mathrm{H}_{26} \mathrm{FeS}_{2}{ }^{+}\left[\mathrm{M}^{+}\right]$: calculated 458.0825, found 458.0824 ${ }^{1} \mathrm{H}$ NMR (400 MHz, $\left.\mathrm{CDCl}_{3}\right)$ $\delta$ 7.44-7.41 (m, 3H), 7.33-7.27 (m, 4H), 7.25-7.20 (m, 8H), 4.37 (dd, $J=10.9,3.2$ $\mathrm{Hz}, 1 \mathrm{H}), 4.16(\mathrm{~s}, 5 \mathrm{H}), 4.12(\mathrm{~d}, J=10.0 \mathrm{~Hz}, 7 \mathrm{H}), 4.02-3.93(\mathrm{~m}, 3 \mathrm{H}) 3.81-3.72(\mathrm{~m}, 1 \mathrm{H}), 3.62-$ $3.53(\mathrm{~m}, 1 \mathrm{H}), 2.32-2.25(\mathrm{~m}, 1 \mathrm{H}), 2.17-2.03(\mathrm{~m}, 2 \mathrm{H}), 1.96-1.89(\mathrm{~m}, 1 \mathrm{H}), 1.38(\mathrm{~d}, J=6.7 \mathrm{~Hz}$, $3 \mathrm{H}), 1.32(\mathrm{~d}, J=6.7 \mathrm{~Hz}, 3 \mathrm{H}) .{ }^{13} \mathrm{C}$ NMR $\left(101 \mathrm{MHz}, \mathrm{CDCl}_{3}\right) \delta 135.4,134.3,133.9,132.5$, $132.0,129.1,129.0,128.8,128.7,127.7,127.6,127.2,126.9,69.0(2 \mathrm{C}), 68.0,67.9,67.5(2 \mathrm{C})$, $67.3,66.4,47.8,47.0,42.3,42.2,41.6,40.5,23.1,20.7$.

\subsection{8 oct-3-en-2-yl(phenyl)sulfane, oct-2-en-1-yl(phenyl)sulfane \& oct-3-en-1- yl(phenyl)sulfane $(20+20 '+20 ")$}

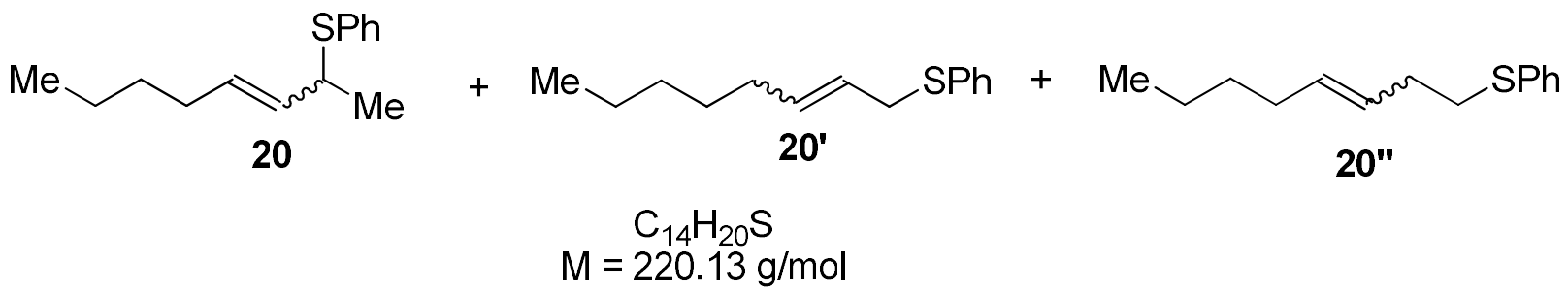

According to GP-A, using $1 \mathrm{~h}$ (15.0 mg, $0.14 \mathrm{mmol}, 1.2$ equiv), 2a (13.0 mg, $0.12 \mathrm{mmol}, 1.0$ equiv), and tris(pentafluorophenyl)borane $\left(B\left(\mathrm{C}_{6} \mathrm{~F}_{5}\right)_{3}\right)(3.0 \mathrm{mg}, 6.0 \mu \mathrm{mol}, 5.0 \mathrm{~mol} \%)$. Purification by column chromatography on silica gel using petroleum ether as eluent. The product 20+20'+20" $\left(21.13 \mathrm{mg}, 80 \%, 20: 20^{\prime}: 20^{\prime \prime}=1: 0.6: 0.5 \mathrm{rr}, 20-E / Z=10: 1,20 '-E / Z=\right.$ $3: 1,20 "-E / Z=1.3: 1$ ) were obtained as colorless liquid mixture. Reaction was also performed by GP-B, using $1 \mathrm{~h}$ (119.0 mg, $1.08 \mathrm{mmol}, 1.2$ equiv), 2a (99.0 mg, $0.90 \mathrm{mmol}, 1.0$ equiv) and Boron trifluoride ethyl etherate $\left(\mathrm{BF}_{3} \cdot \mathrm{Et}_{2} \mathrm{O}\right)(1.3 \mathrm{mg}, 9.0 \mu \mathrm{mol}, 1.0 \mathrm{~mol} \%)$. The products 20+20'+20" were not detected. MSMS (ESI): for $\mathrm{C}_{14} \mathrm{H}_{20} \mathrm{~S}^{+}\left[(\mathrm{M}+\mathrm{H})^{+}\right]$: calculated 221.14, found 221.14. ${ }^{1} \mathrm{H}$ NMR (400 MHz, $\left.\mathrm{CDCl}_{3}\right) \delta$ 7.43-7.32 (m, 5H), 7.30-7.15 (m, 9H), 5.57-5.40 (m, 3H), 5.38-5.28 (m, 3H) 3.77-3.71 (m, 1H), $3.56(\mathrm{~d}, J=6.2 \mathrm{~Hz}, 0.32 \mathrm{H}) .3 .52$ $(\mathrm{d}, J=6.2 \mathrm{~Hz}, 1 \mathrm{H}), 2.96-2.90(\mathrm{~m}, 1 \mathrm{H}), 2.41-2.30(\mathrm{~m}, 1 . \mathrm{H}), 2.02-1.87(\mathrm{~m}, 4 \mathrm{H}), 1.69-1.59$ $(\mathrm{m}, 4 \mathrm{H}), 1.36(\mathrm{~d}, J=6.8 \mathrm{~Hz}, 3 \mathrm{H}) .1 .33-1.14(\mathrm{~m}, 14 \mathrm{H}), 0.91-0.82(\mathrm{~m}, 10 \mathrm{H}) .{ }^{13} \mathrm{C}$ NMR $(101$ $\left.\mathrm{MHz}, \mathrm{CDCl}_{3}\right) \delta 135.3,135.2,134.8,133.0,132.9,132.7,132.2,131.9,131.7,130.0$, 129.2(2C), 128.8, 128.7, 128.6, 127.1, 127.0, 126.9, 126.2, 125.9, 125.9(2C), 51.8, 46.1, $36.7,34.6,33.7,32.4,32.0,31.5,31.4,29.9,29.6,29.0,27.2,22.6,22.6,22.2,20.9,17.8$, $14.2,14.1$. 
yl)(phenyl)sulfane \& (4-cyclohexylbut-3-en-1-yl)(phenyl)sulfane (21+21'+21")

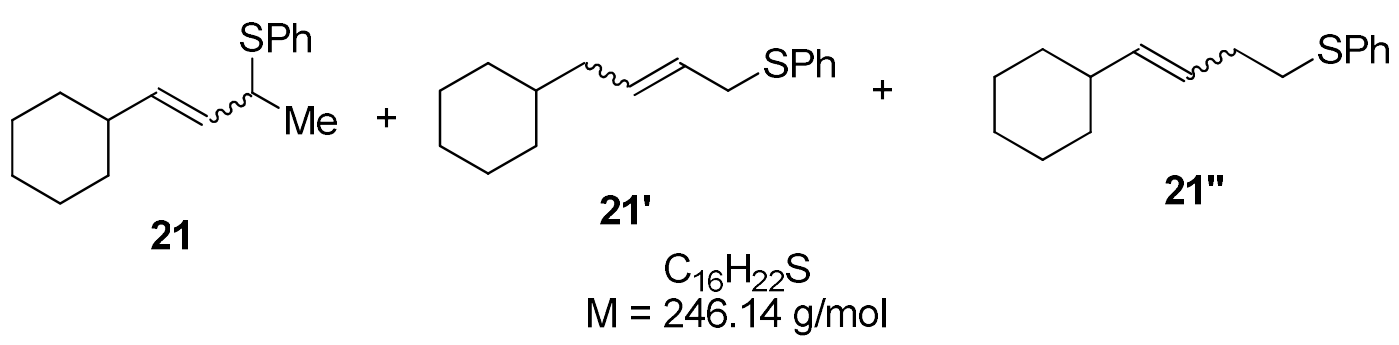

According to GP-A, using $1 \mathbf{i}$ (19.0 mg, $0.14 \mathrm{mmol}, 1.2$ equiv), 2a (13.0 mg, $0.12 \mathrm{mmol}, 1.0$ equiv), and tris(pentafluorophenyl)borane $\left(\mathrm{B}_{(}\left(\mathrm{C}_{6} \mathrm{~F}_{5}\right)_{3}\right)(3.0 \mathrm{mg}, 6.0 \mu \mathrm{mol}, 5.0 \mathrm{~mol} \%)$. Purification by column chromatography on silica gel using petroleum ether as eluent. The products $\mathbf{2 1 + 2 1 ' + 2 1 " ~ ( 1 6 . 0 ~ m g , ~ 5 5 \% , ~} 21: 21^{\prime}: 21^{\prime \prime}=1: 1: 0.75 \mathrm{rr}, 21-E / Z=5: 1,21^{\prime}-E / Z=$ $10: 1,21 "-E / Z=1.3: 1)$ were obtained as colorless liquid mixture. Reaction was also performed by GP-B, using $1 \mathbf{i}$ (147.0 mg, $1.08 \mathrm{mmol}, 1.2$ equiv), 2a (99.0 mg, $0.90 \mathrm{mmol}, 1.0$ equiv) and Boron trifluoride ethyl etherate $\left(\mathrm{BF}_{3} \cdot \mathrm{Et}_{2} \mathrm{O}\right)(1.3 \mathrm{mg}, 9.0 \mu \mathrm{mol}, 1.0 \mathrm{~mol} \%)$. The products 21+21'+21" were not detected. HRMS (ESI): for $\mathrm{C}_{16} \mathrm{H}_{22} \mathrm{~S}^{+}\left[(\mathrm{M}+\mathrm{H})^{+}\right]$: calculated 247.1520, found 247.1506. ${ }^{1} \mathrm{H}$ NMR (400 MHz, $\left.\mathrm{CDCl}_{3}\right) \delta 7.52-7.43(\mathrm{~m}, 1 \mathrm{H}), 7.39-7.32(\mathrm{~m}$, $6 \mathrm{H}), 7.30-7.24(\mathrm{~m}, 8 \mathrm{H}), 7.21-7.15(\mathrm{~m}, 4 \mathrm{H}), 5.54-5.39(\mathrm{~m}, 3 \mathrm{H}), 5.36-5.13(\mathrm{~m}, 5 \mathrm{H}), 3.74-3.67$ $(\mathrm{m}, 1 \mathrm{H}), 3.52(\mathrm{~d}, \mathrm{~J}=6.2 \mathrm{~Hz}, 2 \mathrm{H}), 2.41-2.36(\mathrm{~m}, 1.86 \mathrm{H}), 2.34-2.29(\mathrm{~m}, 1.66 \mathrm{H}), 2.21-2.12(\mathrm{~m}$, $1.47 \mathrm{H}), 1.90-1.83(\mathrm{~m}, 3.30 \mathrm{H}), 1.74-1.62(\mathrm{~m}, 9 \mathrm{H}), 1.36(\mathrm{~d}, J=6.9 \mathrm{~Hz}, 3 \mathrm{H}), 1.28-1.02(\mathrm{~m}$, $18 \mathrm{H}), 0.96-0.86(\mathrm{~m}, 2 \mathrm{H}), 0.83-0.73(\mathrm{~m}, 2 \mathrm{H}) .{ }^{13} \mathrm{C} \mathrm{NMR}\left(101 \mathrm{MHz}, \mathrm{CDCl}_{3}\right) \delta 138.6,138.2$, $137.5,133.3(2 \mathrm{C}), 132.9,130.1,129.2,129.0(2 \mathrm{C}), 128.8,128.6(2 \mathrm{C}), 127.6,127.1,126.8$, 126.2, 125.9, 125.9., 125.2, 58.9, 46.4, 42.2, 40.8, 40.4, 38.0, 36.7, 33.7, 33.4, 33.1(2C), $32.9,32.6,31.5,30.3,27.3,26.6,26.4,26.3,26.2,20.9$. 


\subsection{0 phenyl(6-phenylhex-2-en-1-yl)sulfane and phenyl(6-phenylhex-3-en-1-yl)sulfane $\left(22+222^{\prime}\right)$}

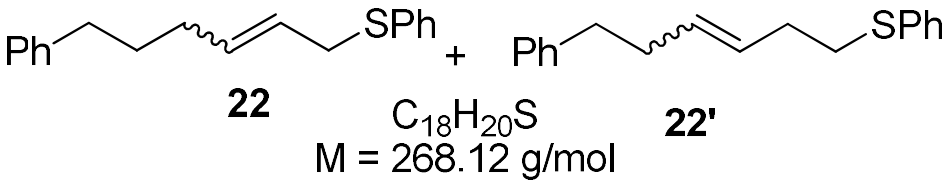

According to GP-A, using $1 \mathbf{j}$ (23.0 mg, $0.14 \mathrm{mmol}, 1.2$ equiv), 2a (13.0 mg, $0.12 \mathrm{mmol}, 1.0$ equiv), and tris(pentafluorophenyl)borane $\left(B\left(\mathrm{C}_{6} \mathrm{~F}_{5}\right)_{3}\right)(3.0 \mathrm{mg}, 6.0 \mu \mathrm{mol}, 5.0 \mathrm{~mol} \%)$. Purification by column chromatography on silica gel using petroleum ether as eluent. The products 22+22' $\left(27.0 \mathrm{mg}, 85 \%, 22: 22^{\prime}=1: 1, \mathrm{rr}, 22^{\prime}-E / Z=3: 1,\right)$ were obtained as colorless liquid mixture. Reaction was also performed by GP-B, using $1 \mathbf{j}$ (171.0 mg, 1.08 mmol, 1.2 equiv), $2 a$ (99.0 mg, $0.90 \mathrm{mmol}, 1.0$ equiv) and Boron trifluoride ethyl etherate $\left(\mathrm{BF}_{3} \cdot \mathrm{Et}_{2} \mathrm{O}\right)(1.3 \mathrm{mg}, 9.0 \mu \mathrm{mol}, 1.0 \mathrm{~mol} \%)$. The products 22+22' were not detected. HRMS (ESI): for $\mathrm{C}_{18} \mathrm{H}_{20} \mathrm{~S}^{+}\left[(\mathrm{M}+\mathrm{H})^{+}\right]$: calculated 269.1364, found 269.1350. ${ }^{1} \mathrm{H}$ NMR (400 MHz, $\left.\mathrm{CDCl}_{3}\right)$ ठ 7.37-7.27 (m, 12H), 7.22-7.14 (m, 8H), 5.99-5.37 (m, 4H), $3.55(\mathrm{~d}, J=5.5 \mathrm{~Hz}, 2 \mathrm{H})$, 2.96-2.93 (m, 1.32H), 2.85-2.81 (m, 0.51H), $2.68(\mathrm{q}, J=7.9 \mathrm{~Hz}, 2 \mathrm{H}), 2.56-2.52(\mathrm{~m}, 2 \mathrm{H})$, $2.34(\mathrm{q}, J=7.0 \mathrm{~Hz}, 3.70 \mathrm{H}), 2.04(\mathrm{q}, J=6.9 \mathrm{~Hz}, 2 \mathrm{H}), 1.68-1.61(\mathrm{~m}, 2 \mathrm{H}) .{ }^{13} \mathrm{C}$ NMR $(101 \mathrm{MHz}$, $\left.\mathrm{CDCl}_{3}\right) \delta 142.4,142.0,136.8,136.2,134.0,132.9,131.6,130.8,129.9,129.2,129.0,128.8$, $128.6(2 \mathrm{C}), 128.5,128.4,126.2,125.9,125.8,125.6,36.6,36.0,35.2$, 34.5, 33.6, 32.4, 31.8, 30.9 .

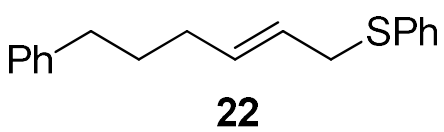

${ }^{1} \mathrm{H}$ NMR $\left(400 \mathrm{MHz}, \mathrm{CDCl}_{3}\right)$ ठ 7.35-7.27 (m, 5H), 7.24-7.10 (m, 5H), 5.58-5.46 (m, 2H), 3.52 (d, $J=5.7 \mathrm{~Hz}, 2 \mathrm{H}), 2.52(\mathrm{t}, J=8.4 \mathrm{~Hz}, 2 \mathrm{H}), 2.01$ (q, $J=6.6 \mathrm{~Hz}, 2 \mathrm{H}), 1.64-1.60(\mathrm{~m}, 2 \mathrm{H}) .{ }^{13} \mathrm{C}$ NMR $\left(101 \mathrm{MHz}, \mathrm{CDCl}_{3}\right) \delta 142.5,136.3,134.1,130.0,128.9,128.6,128.4,126.2,125.8$, 125.6, 36.6, 35.3, 31.8, 30.9.

\section{21 (E)-phenyl(4-phenylpent-3-en-2-yl)sulfane (23)}

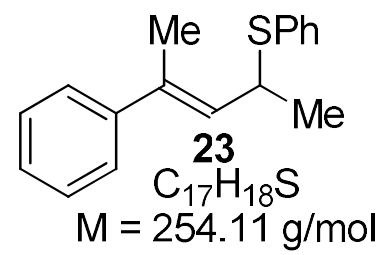

According to GP-A, using 1k (21.0 mg, 0.14 mmol, 1.2 equiv), 2a (13.0 mg, 0.12 mmol, 1.0 equiv), and tris(pentafluorophenyl)borane $\left(\mathrm{B}_{(}\left(\mathrm{C}_{6} \mathrm{~F}_{5}\right)_{3}\right)(6.0 \mathrm{mg}, 12.0 \mu \mathrm{mol}, 10.0 \mathrm{~mol} \%)$. 
Purification by column chromatography on silica gel using petroleum ether as eluent. The product 23 (30.0 mg, 98\%, E/Z = 4:1) was obtained as colorless liquid. Reaction was also performed by GP-B, using 1k (155.0 mg, 1.08 mmol, 1.2 equiv), 2a (99.0 mg, 0.90 mmol, 1.0 equiv) and Boron trifluoride ethyl etherate $\left(\mathrm{BF}_{3} \cdot \mathrm{Et}_{2} \mathrm{O}\right)(1.3 \mathrm{mg}, 9.0 \mu \mathrm{mol}, 1.0 \mathrm{~mol} \%)$. Purification by column chromatography on silica gel using petroleum ether as eluent. The product 23 (151.0 mg, 66\%). MS-MS (ESI): for $\mathrm{C}_{17} \mathrm{H}_{18} \mathrm{~S}^{+}\left[(\mathrm{M}+\mathrm{H})^{+}\right]$: calculated 255.12, found 255.09 along with the other fragments $240.10,128.06,105.07,91.05 .{ }^{1} \mathbf{H}$ NMR $(400 \mathrm{MHz}$, $\left.\mathrm{CDCl}_{3}\right) \delta 7.49-7.46(\mathrm{~m}, 2 \mathrm{H}), 7.31(\mathrm{~d}, J=4.2 \mathrm{~Hz}, 4 \mathrm{H}), 7.29-7.22(\mathrm{~m}, 4 \mathrm{H}), 5.66(\mathrm{dd}, J=10.1$, $1.7 \mathrm{~Hz}, 1 \mathrm{H}), 4.20-4.13(\mathrm{~m}, 1 \mathrm{H}), 1.76(\mathrm{~d}, J=1.6 \mathrm{~Hz}, 3 \mathrm{H}), 1.44(\mathrm{~d}, J=6.6 \mathrm{~Hz}, 3 \mathrm{H}) .{ }^{13} \mathrm{C}$ NMR $\left(101 \mathrm{MHz}, \mathrm{CDCl}_{3}\right) \delta 143.3,136.1,134.7,134.4,130.4,128.8,128.3,127.7,127.1,125.9$, $43.1,21.4,16.0$.

\subsection{2 (4,4-diphenylbut-3-en-2-yl)(phenyl)sulfane (24)}

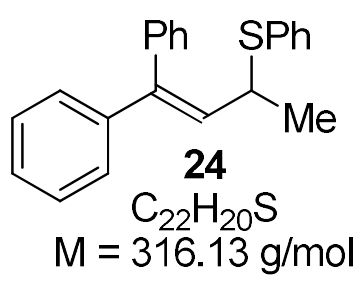

According to GP-A, using 1 ( $(154.0 \mathrm{mg}, 0.69 \mathrm{mmol}, 1.2$ equiv), 2a (64.0 mg, $0.58 \mathrm{mmol}, 1.0$ equiv), and tris(pentafluorophenyl)borane $\left(\mathrm{B}_{(}\left(\mathrm{C}_{6} \mathrm{~F}_{5}\right)_{3}\right)(3.0 \mathrm{mg}, 5.8 \mu \mathrm{mol}, 1.0 \mathrm{~mol} \%)$ Purification by column chromatography on silica gel using petroleum ether as eluent. The product 24 (182.0 mg, 99\%) was obtained as colorless liquid. Reaction was also performed by GP-B, using 1 ( $(221.0 \mathrm{mg}, 1.08 \mathrm{mmol}, 1.2$ equiv), 2a (99.0 mg, $0.90 \mathrm{mmol}, 1.0$ equiv) and Boron trifluoride ethyl etherate $\left(\mathrm{BF}_{3} \mathrm{Et}_{2} \mathrm{O}\right)(1.3 \mathrm{mg}, 9.0 \mu \mathrm{mol}, 1.0 \mathrm{~mol} \%)$. Purification by column chromatography on silica gel using petroleum ether as eluent. The product 24 (216.0 mg, 76\%) was obtained as colorless liquid. MS-MS (ESI): for $\mathrm{C}_{22} \mathrm{H}_{20} \mathrm{~S}^{+}\left[(\mathrm{M}+\mathrm{H})^{+}\right]$: calculated 317.14, found 317.15. ${ }^{1} \mathrm{H}$ NMR $\left(400 \mathrm{MHz}, \mathrm{CDCl}_{3}\right) \delta 7.37-7.32(\mathrm{~m}, 5 \mathrm{H}), 7.30-7.25(\mathrm{~m}, 6 \mathrm{H})$, 7.19-7.17 (m, 2H), 6.96-6.93 (m, 2H), $6.04(\mathrm{~d}, J=10.6 \mathrm{~Hz}, 1 \mathrm{H}), 4.04-3.97(\mathrm{~m}, 1 \mathrm{H}), 1.52(\mathrm{~d}$, $J=6.8 \mathrm{~Hz}, 3 \mathrm{H}) .{ }^{13} \mathrm{C}$ NMR $\left(101 \mathrm{MHz}, \mathrm{CDCl}_{3}\right) \delta 142.0,139.3,134.4,133.7,131.5,129.8$, 128.7, 128.2(2C), 127.5, $127.4(3 \mathrm{C}), 127.3,43.5,21.4$. 


\subsection{3 (1-cyclohexylidenepropan-2-yl)(phenyl)sulfane (25)}

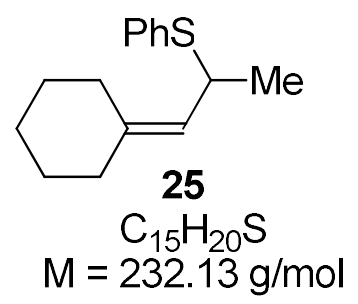

According to GP-A, using $1 \mathrm{~m}$ (19.0 mg, $0.14 \mathrm{mmol}, 1.2$ equiv), 2a (13.0 mg, $0.12 \mathrm{mmol}, 1.0$ equiv), and tris(pentafluorophenyl)borane $\left(\mathrm{B}_{(}\left(\mathrm{C}_{6} \mathrm{~F}_{5}\right)_{3}\right)(3.0 \mathrm{mg}, 6.0 \mu \mathrm{mol}, 5.0 \mathrm{~mol} \%)$. Purification by column chromatography on silica gel using petroleum ether as eluent. The product 25 (27.0 mg, 96\%) was obtained as colorless liquid. Reaction was also performed by GP-B, using $1 \mathrm{~m}$ (147.0 mg, $1.08 \mathrm{mmol}, 1.2$ equiv), 2a (99.0 mg, $0.90 \mathrm{mmol}, 1.0$ equiv) and Boron trifluoride ethyl etherate $\left(\mathrm{BF}_{3} \cdot \mathrm{Et}_{2} \mathrm{O}\right)(1.3 \mathrm{mg}, 9.0 \mu \mathrm{mol}, 1.0 \mathrm{~mol} \%)$. Purification by column chromatography on silica gel using petroleum ether as eluent. The product 25 (27.0 $\mathrm{mg}, 13 \%$ ) was obtained as colorless liquid. MS-MS (ESI): for $\mathrm{C}_{15} \mathrm{H}_{20} \mathrm{~S}^{+}\left[(\mathrm{M}+\mathrm{H})^{+}\right]$: calculated 233.14, found 233.12. ${ }^{1} \mathrm{H}$ NMR $\left(400 \mathrm{MHz}, \mathrm{CDCl}_{3}\right) \delta 7.42-7.39(\mathrm{~m}, 2 \mathrm{H}), 7.29-7.21(\mathrm{~m}, 3 \mathrm{H})$, $5.01(\mathrm{~d}, J=10.2 \mathrm{~Hz}, 1 \mathrm{H}), 4.11-4.04(\mathrm{~m}, 1 \mathrm{H}), 2.01(\mathrm{dd}, J=7.1,4.8 \mathrm{~Hz}, 2 \mathrm{H}), 1.96-1.89(\mathrm{~m}$, $2 \mathrm{H}), 1.52-1.34(\mathrm{~m}, 6 \mathrm{H}), 1.32(\mathrm{~d}, J=6.6 \mathrm{~Hz}, 3 \mathrm{H}) .{ }^{13} \mathrm{C} \mathrm{NMR}\left(101 \mathrm{MHz}, \mathrm{CDCl}_{3}\right) \delta 141.5,135.4$, $133.7,128.7,127.2,124.1,41.4,37.1,29.1,28.5,27.6,26.8,21.8$.

\subsection{4 (E)-phenyl(1-phenylpent-1-en-3-yl)sulfane (26)}

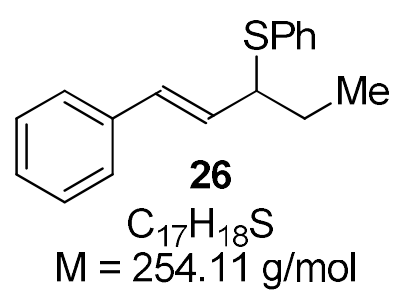

According to GP-A, using 1n (21.0 mg, 0.14 mmol, 1.2 equiv), 2a (13.0 mg, 0.12 mmol, 1.0 equiv), and tris(pentafluorophenyl)borane $\left(\mathrm{B}_{(}\left(\mathrm{C}_{6} \mathrm{~F}_{5}\right)_{3}\right)(3.0 \mathrm{mg}, 6.0 \mu \mathrm{mol}, 5.0 \mathrm{~mol} \%)$. Purification by column chromatography on silica gel using petroleum ether as eluent. The product 26 (16.0 mg, 52\%) was obtained as colorless liquid. Reaction was also performed by GP-B, using 1n (156.0 mg, $1.08 \mathrm{mmol}, 1.2$ equiv), 2a (99.0 mg, $0.90 \mathrm{mmol}, 1.0$ equiv) and Boron trifluoride ethyl etherate $\left(\mathrm{BF}_{3} \cdot \mathrm{Et}_{2} \mathrm{O}\right)(1.3 \mathrm{mg}, 9.0 \mu \mathrm{mol}, 1.0 \mathrm{~mol} \%)$ The product 26 was not detected. MS-MS (ESI): for $\mathrm{C}_{17} \mathrm{H}_{18} \mathrm{~S}^{+}\left[(\mathrm{M}+\mathrm{H})^{+}\right]$: calculated 255.12, found 255.09 along with the other fragments $240.09,130.07,129.07,128.06,115.05,91.05 .{ }^{1} \mathbf{H}$ NMR $(400 \mathrm{MHz}$, $\left.\mathrm{CDCl}_{3}\right) \delta 7.42(\mathrm{dd}, J=8.0,1.7 \mathrm{~Hz}, 2 \mathrm{H}), 7.29-7.20(\mathrm{~m}, 8 \mathrm{H}), 6.18(\mathrm{~d}, J=15.9 \mathrm{~Hz}, 1 \mathrm{H}), 6.06$ (dd, $J=15.8,8.9 \mathrm{~Hz}, 1 \mathrm{H}), 3.68-3.62(\mathrm{~m}, 1 \mathrm{H}), 1.90-1.82(\mathrm{~m}, 1 \mathrm{H}), 1.78-1.68(\mathrm{~m}, 1 \mathrm{H}), 1.06(\mathrm{t}, J$ 
$=7.4 \mathrm{~Hz}, 3 \mathrm{H}) .{ }^{13} \mathrm{C}$ NMR $\left(101 \mathrm{MHz}, \mathrm{CDCl}_{3}\right) \delta 137.0,134.6,133.5,131.2,130.6,128.8,128.6$, $127.5,127.4,126.4,54.1,28.0,12.2$.

\section{$6.25(E)$-phenyl(1-phenyloct-1-en-3-yl)sulfane (27)}

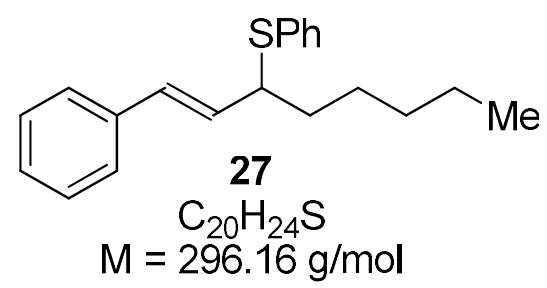

According to GP-A, using 10 (27.0 mg, $0.14 \mathrm{mmol}, 1.2$ equiv), 2a (13.0 mg, $0.12 \mathrm{mmol}, 1.0$

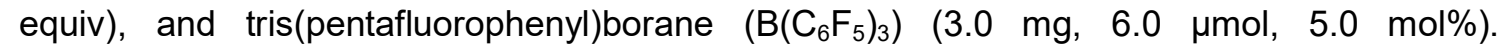
Purification by column chromatography on silica gel using petroleum ether as eluent. The product 27 (23.0 mg, 66\%) was obtained as colorless liquid. Reaction was also performed by GP-B, using 10 (201.0 mg, $1.08 \mathrm{mmol}, 1.2$ equiv), 2a (99.0 mg, $0.90 \mathrm{mmol}, 1.0$ equiv) and Boron trifluoride ethyl etherate $\left(\mathrm{BF}_{3} \cdot \mathrm{Et}_{2} \mathrm{O}\right)(1.3 \mathrm{mg}, 9.0 \mu \mathrm{mol}, 1.0 \mathrm{~mol} \%)$ The product 27 was not detected. MS-MS (ESI): for $\mathrm{C}_{20} \mathrm{H}_{24} \mathrm{~S}^{+}\left[(\mathrm{M}+\mathrm{H})^{+}\right]$: calculated 297.17, found 297.10 along with the other fragments $167.04,129.04,117.04,91.05 .{ }^{1} \mathbf{H} \mathbf{N M R}\left(400 \mathrm{MHz}, \mathrm{CDCl}_{3}\right) \delta 7.40$ $7.37(\mathrm{~m}, 2 \mathrm{H}), 7.27-7.17(\mathrm{~m}, 8 \mathrm{H}), 6.14-6.00(\mathrm{~m}, 2 \mathrm{H}), 3.72-3.66(\mathrm{~m}, 1 \mathrm{H}), 1.83-1.63(\mathrm{~m}, 2 \mathrm{H})$, 1.48-1.41 (m, 2H), 1.30-1.26 (m, 4H), 0.89-0.82 (m, 3H). ${ }^{13} \mathrm{C}$ NMR (101 MHz, CDCl $\left.{ }_{3}\right) \delta$ $137.1,134.7,133.5,131.0,130.9,128.8,128.6,127.5,127.4,126.4,52.6,34.8,31.7,27.3$, 22.7, 14.2 .

\subsection{6 (E)-(2-methyl-4-phenylbut-3-en-2-yl)(phenyl)sulfane (28)}

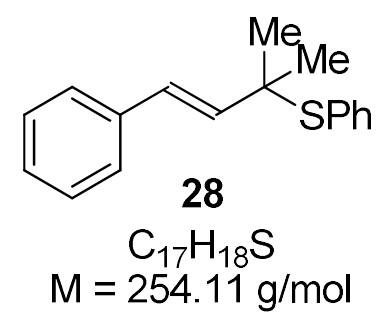

According to GP-A, using $1 \mathrm{p}$ (100.0 mg, $0.69 \mathrm{mmol}, 1.2$ equiv), 2a (64.0 mg, $0.58 \mathrm{mmol}, 1.0$ equiv), and tris(pentafluorophenyl)borane $\left(B\left(\mathrm{C}_{6} \mathrm{~F}_{5}\right)_{3}\right) \quad(3.0 \mathrm{mg}, 5.8 \mu \mathrm{mol}, 1.0 \mathrm{~mol} \%)$ Purification by column chromatography on silica gel using petroleum ether as eluent. The product 28 (130.0 mg, 88\%) was obtained as colorless liquid. Reaction was also performed by GP-B, using 1p (156.0 mg, $1.08 \mathrm{mmol}, 1.2$ equiv), 2a (99.0 mg, $0.90 \mathrm{mmol}, 1.0$ equiv) and Boron trifluoride ethyl etherate $\left(\mathrm{BF}_{3} \mathrm{Et}_{2} \mathrm{O}\right)(1.3 \mathrm{mg}, 9.0 \mu \mathrm{mol}, 1.0 \mathrm{~mol} \%)$ The product 28 was not detected. MS-MS (ESI): for $\mathrm{C}_{17} \mathrm{H}_{18} \mathrm{~S}^{+}\left[(\mathrm{M}+\mathrm{H})^{+}\right]$: calculated 255.12, found 255.11 along with the other fragments $240.09,179.08,129.07,128.06,91.05 .{ }^{1} \mathrm{H}$ NMR $\left(400 \mathrm{MHz}, \mathrm{CDCl}_{3}\right) \delta$ 7.45-7.42 (m, 2H), 7.30-7.28 (m, 5H), 7.24-7.20 (m, 3H), $6.31(\mathrm{~d}, J=16.1 \mathrm{~Hz}, 1 \mathrm{H}), 5.98(\mathrm{~d}$, 
$J=16.1 \mathrm{~Hz}, 1 \mathrm{H}), 1.45(\mathrm{~s}, 6 \mathrm{H}) .{ }^{13} \mathrm{C}$ NMR $\left(101 \mathrm{MHz}, \mathrm{CDCl}_{3} \delta 137.6,137.3,136.8,132.4\right.$, $128.9,128.7,128.5,127.4,127.1,126.4,50.2,28.0$.

\subsection{7 (E)-(3-methyl-1-phenylpent-1-en-3-yl)(phenyl)sulfane (29)}

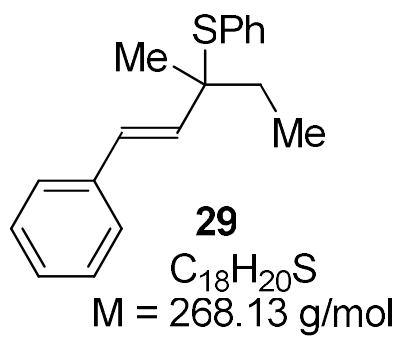

According to GP-A, using 1q (110.0 mg, $0.69 \mathrm{mmol}, 1.2$ equiv), 2a (64.0 mg, $0.58 \mathrm{mmol}, 1.0$

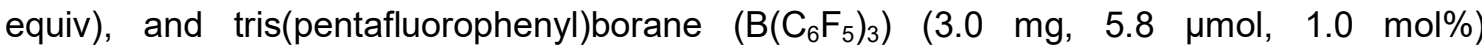
Purification by column chromatography on silica gel using petroleum ether as eluent. The product 29 (128.0 mg, 82\%) was obtained as colorless liquid. Reaction was also performed by GP-B, using 1q (178.0 mg, $1.08 \mathrm{mmol}, 1.2$ equiv), 2a (99.0 mg, $0.90 \mathrm{mmol}, 1.0$ equiv) and Boron trifluoride ethyl etherate $\left(\mathrm{BF}_{3} \cdot \mathrm{Et}_{2} \mathrm{O}\right)(1.3 \mathrm{mg}, 9.0 \mu \mathrm{mol}, 1.0 \mathrm{~mol} \%)$. The product 29 was not detected. MS-MS (ESI): for $\mathrm{C}_{18} \mathrm{H}_{20} \mathrm{~S}^{+}\left[(\mathrm{M}+\mathrm{H})^{+}\right]$: calculated 269.14, found 269.13 along with the other fragments $255.12,158.10,143.08,129.07,91.05 .{ }^{1} \mathrm{H}$ NMR $\left(400 \mathrm{MHz}, \mathrm{CDCl}_{3}\right) \delta$ 7.45-7.42 (m, 2H), 7.31-7.30 (m, 4H), 7.26-7.21 (m, 4H), 6.25 (d, J = 16.1 Hz, 1H), 5.96 (d, $J=16.3 \mathrm{~Hz}, 1 \mathrm{H}), 1.81-1.72(\mathrm{~m}, 2 \mathrm{H}), 1.38(\mathrm{~s}, 3 \mathrm{H}), 1.00(\mathrm{t}, J=7.5 \mathrm{~Hz}, 3 \mathrm{H}) .{ }^{13} \mathrm{C}$ NMR $(101$ $\left.\mathrm{MHz}_{\mathrm{CDCl}}\right) \delta 137.7,137.4,135.7,132.1,128.8,128.7,128.4,128.1,127.3,126.3,54.4$, $33.7,23.4,9.4$.

\subsection{8 (E)-(3-methyl-1-phenyloct-1-en-3-yl)(phenyl)sulfane (30)}

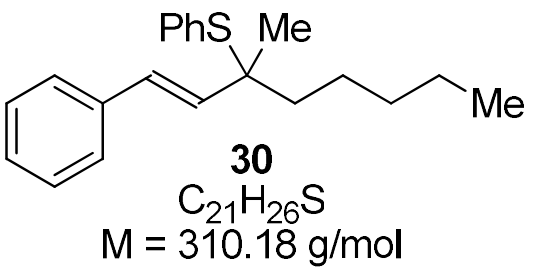

According to GP-A, using 1r (139.0 mg, $0.69 \mathrm{mmol}, 1.2$ equiv), 2a (64.0 mg, $0.58 \mathrm{mmol}, 1.0$ equiv), and tris(pentafluorophenyl)borane $\left(B\left(\mathrm{C}_{6} \mathrm{~F}_{5}\right)_{3}\right) \quad(3.0 \mathrm{mg}, 5.8 \mu \mathrm{mol}, 1.0 \mathrm{~mol} \%)$. Purification by column chromatography on silica gel using petroleum ether as eluent. The product 30 (92.0 mg, 51\%) was obtained as colorless liquid. Reaction was also performed by GP-B, using 1r (216.0 mg, $1.08 \mathrm{mmol}, 1.2$ equiv), 2a (99.0 mg, $0.90 \mathrm{mmol}, 1.0$ equiv) and Boron trifluoride ethyl etherate $\left(\mathrm{BF}_{3} \cdot \mathrm{Et}_{2} \mathrm{O}\right)(1.3 \mathrm{mg}, 9.0 \mu \mathrm{mol}, 1.0 \mathrm{~mol} \%)$. The product 30 was not detected. MS-MS (ESI): for $\mathrm{C}_{21} \mathrm{H}_{26} \mathrm{~S}^{+}\left[(\mathrm{M}+\mathrm{H})^{+}\right]$: calculated 311.18 , found 311.18 along with the other fragments $143.08,130.07,129.07,91.05 .{ }^{1} \mathrm{H}$ NMR $\left(400 \mathrm{MHz}, \mathrm{CDCl}_{3}\right) \delta 7.42-$ 
$7.40(\mathrm{~m}, 2 \mathrm{H}), 7.30-7.29(\mathrm{~m}, 5 \mathrm{H}), 7.25-7.20(\mathrm{~m}, 3 \mathrm{H}), 6.25(\mathrm{~d}, J=16.1 \mathrm{~Hz}, 1 \mathrm{H}), 5.92(\mathrm{~d}, J=$ $16.1 \mathrm{~Hz}, 1 \mathrm{H}), 1.73-1.68(\mathrm{~m}, 2 \mathrm{H}), 1.51-1.41(\mathrm{~m}, 2 \mathrm{H}), 1.38(\mathrm{~s}, 3 \mathrm{H}), 1.32-1.26(\mathrm{~m}, 4 \mathrm{H}), 0.88(\mathrm{t}$, $J=6.9 \mathrm{~Hz}, 3 \mathrm{H}) .{ }^{13} \mathrm{C}$ NMR $\left(101 \mathrm{MHz}, \mathrm{CDCl}_{3}\right) \delta 137.7,137.4,136.0,132.1,128.8,128.7$, $128.4,127.8,127.3,126.3,54.1,41.2,32.4,24.7,24.0,22.7,14.2$.

\subsection{9 (E)-(2-methyl-1,4-diphenylbut-3-en-2-yl)(phenyl)sulfane (31)}

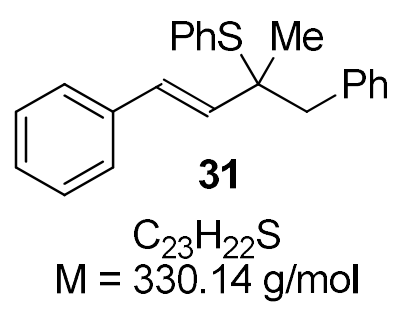

According to GP-A, using 1s (153.0 mg, 0.69 mmol, 1.2 equiv), 2a (64.0 mg, 0.58 mmol, 1.0

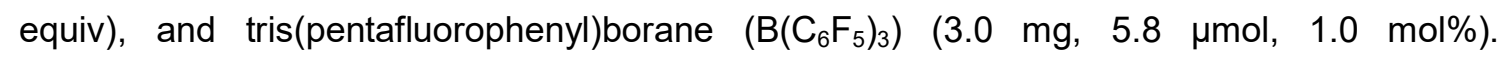
Purification by column chromatography on silica gel using petroleum ether as eluent. The product 31 (65.0 mg, 39\%) was obtained as colorless liquid. Reaction was also performed by GP-B, using 1s (238.0 mg, $1.08 \mathrm{mmol}, 1.2$ equiv), 2a (99.0 mg, $0.90 \mathrm{mmol}, 1.0$ equiv) and Boron trifluoride ethyl etherate $\left(\mathrm{BF}_{3} \cdot \mathrm{Et}_{2} \mathrm{O}\right)(1.3 \mathrm{mg}, 9.0 \mu \mathrm{mol}, 1.0 \mathrm{~mol} \%)$ The product 31 was not detected. MS-MS (ESI): for $\mathrm{C}_{23} \mathrm{H}_{22} \mathrm{~S}^{+}\left[(\mathrm{M}+\mathrm{H})^{+}\right]$: calculated 331.15, found 331.13. ${ }^{1} \mathbf{H}$ NMR (400 MHz, $\left.\mathrm{CDCl}_{3}\right) \delta$ 7.46-7.44 (m, 2H), 7.32-7.30 (m, 2H), 7.28-7.27 (m, 3H), 7.25-7.18 (m, $8 \mathrm{H}), 6.39(\mathrm{~d}, J=16.1 \mathrm{~Hz}, 1 \mathrm{H}), 5.83(\mathrm{~d}, J=16.1 \mathrm{~Hz}, 1 \mathrm{H}), 3.18(\mathrm{~d}, J=13.4 \mathrm{~Hz}, 1 \mathrm{H}), 3.03(\mathrm{~d}, J$ $=13.4 \mathrm{~Hz}, 1 \mathrm{H}), 1.33(\mathrm{~s}, 3 \mathrm{H}) \cdot{ }^{13} \mathrm{C}$ NMR $\left(101 \mathrm{MHz}, \mathrm{CDCl}_{3}\right) \delta 137.8,137.3,137.1,135.2,133.7$, $131.8,130.8,129.0,128.7,128.5,128.0,127.4,126.7,126.4,77.5,48.2,23.7$. 
yl)(phenyl)sulfane (32')

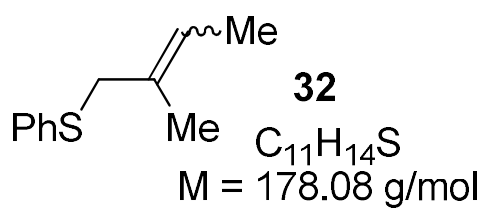

According to GP-A, using 1t (47.0 mg, $0.69 \mathrm{mmol}, 1.2$ equiv), 2a (64.0 mg, $0.58 \mathrm{mmol}, 1.0$

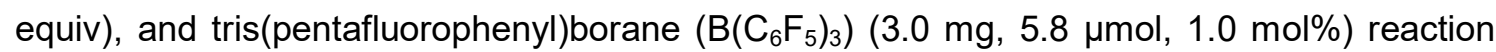
performed at $-10^{\circ} \mathrm{C}$. Purification by column chromatography on silica gel using petroleum ether as eluent. The products $32+32^{\prime}(83.0 \mathrm{mg}, 90 \%, E / Z=10: 1, r r=5: 1)$ were obtained as colorless liquid mixture. Reaction was also performed by GP-B, using 1t (74.0 mg, 1.08 mmol, 1.2 equiv), 2a (99.0 mg, $0.90 \mathrm{mmol}, 1.0$ equiv) and Boron trifluoride ethyl etherate $\left(\mathrm{BF}_{3} \cdot \mathrm{Et}_{2} \mathrm{O}\right)(1.3 \mathrm{mg}, 9.0 \mu \mathrm{mol}, 1.0 \mathrm{~mol} \%)$. Purification by column chromatography on silica gel using petroleum ether as eluent. The product $32^{\prime}(48.0 \mathrm{mg}, 30 \%)$ was obtained as colorless liquid. ${ }^{1} \mathrm{H}$ NMR $\left(400 \mathrm{MHz}, \mathrm{CDCl}_{3}\right) \delta 7.37-7.26(\mathrm{~m}, 4 \mathrm{H}), 7.20-7.12(\mathrm{~m}, 1 \mathrm{H}), 5.33$ (q, J=7.0 $\mathrm{Hz}, 1 \mathrm{H}), 3.51(\mathrm{~s}, 2 \mathrm{H}), 1.74(\mathrm{~s}, 3 \mathrm{H}), 1.56(\mathrm{~d}, \mathrm{~J}=8.5 \mathrm{~Hz}, 3 \mathrm{H}) \cdot{ }^{13} \mathrm{C}$ NMR $\left(101 \mathrm{MHz}, \mathrm{CDCl}_{3}\right) \delta$ $136.9,131.1,130.2,128.8,126.2,123.3,44.2,15.1,13.5$.

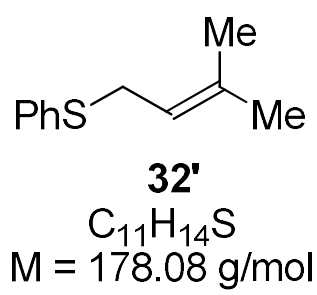

${ }^{1} \mathrm{H}$ NMR $\left(400 \mathrm{MHz}, \mathrm{CDCl}_{3}\right) \delta 7.33(\mathrm{~d}, J=7.2 \mathrm{~Hz}, 2 \mathrm{H}), 7.28-7.24(\mathrm{~m}, 2 \mathrm{H}), 7.19-7.15(\mathrm{~m}, 1 \mathrm{H})$, 5.32-5.28 (m, 1H), $3.54(\mathrm{~d}, J=7.7 \mathrm{~Hz}, 2 \mathrm{H}), 1.71(\mathrm{~s}, 3 \mathrm{H}), 1.58(\mathrm{~s}, 3 \mathrm{H}) .{ }^{13} \mathrm{C}$ NMR $(101 \mathrm{MHz}$, $\left.\mathrm{CDCl}_{3}\right) \delta 136.9,136.6,129.8,128.9,126.1,119.4,32.3,25.8,17.8$.

\subsection{1 (2-ethylidene-6-methylhept-5-en-1-yl)(phenyl)sulfane (33)}

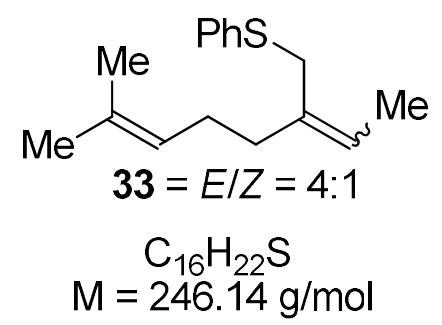

According to GP-A, using 1u (95.0 mg, $0.69 \mathrm{mmol}, 1.2$ equiv), 2a (64.0 mg, $0.58 \mathrm{mmol}, 1.0$ equiv), and tris(pentafluorophenyl)borane $\left(\mathrm{B}_{(}\left(\mathrm{C}_{6} \mathrm{~F}_{5}\right)_{3}\right)(3.0 \mathrm{mg}, 5.8 \mu \mathrm{mol}, 1.0 \mathrm{~mol} \%)$. Purification by column chromatography on silica gel using petroleum ether as eluent. The product $33(121.0 \mathrm{mg}, 85 \%, E / Z=4: 1)$ was obtained as colorless liquid. Reaction was also 
performed by GP-B, using 1u (147.0 mg, $1.08 \mathrm{mmol}, 1.2$ equiv), 2a (99.0 mg, $0.90 \mathrm{mmol}, 1.0$ equiv) and Boron trifluoride ethyl etherate $\left(\mathrm{BF}_{3} \cdot \mathrm{Et}_{2} \mathrm{O}\right)(1.3 \mathrm{mg}, 9.0 \mu \mathrm{mol}, 1.0 \mathrm{~mol} \%)$. Purification by column chromatography on silica gel using petroleum ether as eluent. The product 32 was not obtained HRMS (ESI): for $\mathrm{C}_{16} \mathrm{H}_{22} \mathrm{~S}^{+}\left[(\mathrm{M}+\mathrm{H})^{+}\right]$: calculated 247.1520, found 247.1521 ${ }^{1} \mathrm{H}$ NMR $\left(400 \mathrm{MHz}, \mathrm{CDCl}_{3}\right)$ ठ 7.38-7.31 (m, 2H), 7.29-7.24 (m, 2H), 7.19-7.15 (m, 1H), 5.37 (q, $J=6.9 \mathrm{~Hz}, 1 \mathrm{H}), 5.15-5.10(\mathrm{~m}, 1 \mathrm{H}), 3.54(\mathrm{~s}, 2 \mathrm{H}), 2.24-2.18(\mathrm{~m}, 2 \mathrm{H}), 2.13-2.07$ $(\mathrm{m}, 2 \mathrm{H}), 1.69(\mathrm{~s}, 3 \mathrm{H}), 1.62(\mathrm{~s}, 3 \mathrm{H}), 1.58(\mathrm{~d}, J=6.9 \mathrm{~Hz}, 3 \mathrm{H}) \cdot{ }^{13} \mathrm{C}$ NMR (101 MHz, CDCl $)_{3} \delta$ 137.1, 135.0, 132.1, 130.0, 128.8, 126.1, 124.1, 123.9, 41.8, 28.9, 26.8, 25.9, 17.8, 13.6.

\subsection{Cyclohex-2-en-1-yl(phenyl)sulfane (34)}

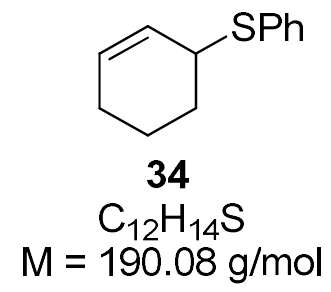

According to GP-A, using 1v (56.0 mg, 0.69 mmol, 1.2 equiv), 2a (64.0 mg, 0.58 mmol, 1.0 equiv), and tris(pentafluorophenyl)borane $\left(\mathrm{B}_{(}\left(\mathrm{C}_{6} \mathrm{~F}_{5}\right)_{3}\right)(3.0 \mathrm{mg}, 5.8 \mu \mathrm{mol}, 1.0 \mathrm{~mol} \%)$ Purification by column chromatography on silica gel using petroleum ether as eluent. The product 34 (110.0 mg, 91\%) was obtained as colorless liquid. Reaction was also performed by GP-B, using $1 \mathbf{v}(87.0 \mathrm{mg}, 1.08 \mathrm{mmol}, 1.2$ equiv), 2a (99.0 mg, $0.90 \mathrm{mmol}, 1.0$ equiv) and Boron trifluoride ethyl etherate $\left(\mathrm{BF}_{3} \cdot \mathrm{Et}_{2} \mathrm{O}\right)(1.3 \mathrm{mg}, 9.0 \mu \mathrm{mol}, 1.0 \mathrm{~mol} \%)$. The product 34 was not detected. MS-MS (ESI): for $\mathrm{C}_{16} \mathrm{H}_{16} \mathrm{~S}^{+}\left[(\mathrm{M}+\mathrm{H})^{+}\right]$: calculated 191.09, found 191.05 . ${ }^{1} \mathrm{H}$ NMR $\left(400 \mathrm{MHz}, \mathrm{CDCl}_{3}\right)$ ठ 7.43-7.40 (m, 2H), 7.31-7.27 (m, 2H), 7.24-7.19 (m, 1H), 5.86-5.82 (m, 1H), 5.79-5.75 (m, 1H), 3.88-3.84 (m, 1H), 2.08-2.00 (m, 2H), 1.97-1.86 (m, 2H), 1.82-1.76 $(\mathrm{m}, 1 \mathrm{H}), 1.66-1.59(\mathrm{~m}, 1 \mathrm{H}) .{ }^{13} \mathrm{C}$ NMR $\left(101 \mathrm{MHz}, \mathrm{CDCl}_{3}\right) \delta 136.0,131.4,130.6,129.0,127.0$, $126.7,44.0,28.9,25.1,19.6$. 


\subsection{Gram scale synthesis}

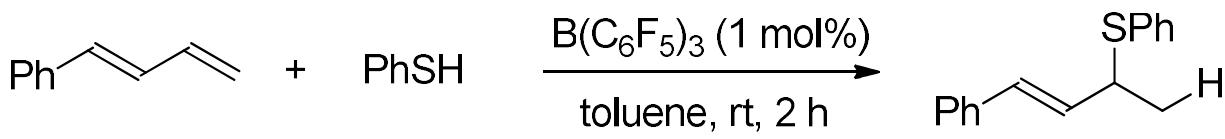

$$
\text { (1.2 equiv) (1.0 equiv) }
$$

Gram scale synthesis has been done by using the GP-A, 1a $(1310.0 \mathrm{mg}, 10.08 \mathrm{mmol}$, 1.2 equiv), 2a (924.0 mg, $8.4 \mathrm{mmol}, 1.0$ equiv), and tris(pentafluorophenyl)borane $\left(B\left(C_{6} F_{5}\right)_{3}\right)(43.0 \mathrm{mg}, 0.084 \mathrm{mmol}, 1.0 \mathrm{~mol} \%)$. Purification by column chromatography on silica gel using petroleum ether as eluent. The product 3 (1.56 g, 93\%) was obtained as colorless liquid.

\subsection{Derivatization of the sulfide to sulfone}

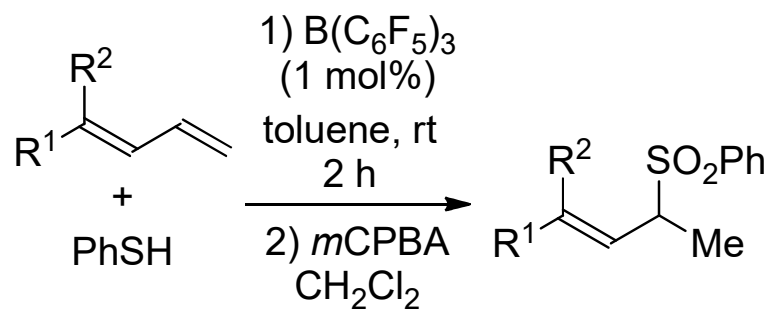

\section{Procedure 1}

First step has been done by following the GP-A, after isolation of the sulfide, oxidation was carried out via reported method in the literature. ${ }^{[\mathrm{S10}]}$ To a stirred solution of sulfane $(0.1 \mathrm{mmol}$, 1.0 equiv) in $1 \mathrm{ml}$ DCM was added $m$ CPBA $\left(0.2 \mathrm{mmol}, 2\right.$ equiv) at $0{ }^{\circ} \mathrm{C}$, reaction kept for stirring for 4 hours at the same temperature. After completion the reaction was quenched with aqueous solution of $\mathrm{NaHCO}_{3}$, extracted with ethyl acetate, and the organic layer passed over anhydrous $\mathrm{Na}_{2} \mathrm{SO}_{4}$. Pure compound was isolated by column chromatography on silica gel using petroleum ether/ethyl acetate as eluent afforded the respected products.

\section{1 (E)-((4-phenylbut-3-en-2-yl)sulfonyl)benzene (35)}

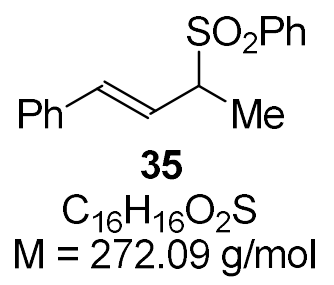

Prepared according to the procedure 1 , by using $3(24.0 \mathrm{mg}, 0.1 \mathrm{mmol}, 1.0$ equiv), $m$ CPBA (34 mg, $0.2 \mathrm{mmol}$, 2equiv) in $\mathrm{DCM}$ at $0{ }^{\circ} \mathrm{C}$ for 4 hours. Purification by column chromatography on silica gel using petroleum ether/ethyl acetate (10:1) as eluent. The product 35 (20.0 mg, 73\%) was obtained as white solid. M.P - 60-61 ${ }^{\circ} \mathrm{C}$ HRMS (ESI): for 
$\mathrm{C}_{16} \mathrm{H}_{16} \mathrm{O}_{2} \mathrm{~S}^{+}\left[(\mathrm{M}+\mathrm{Na})^{+}\right]$: calculated 295.0769, found 295.0756. ${ }^{1} \mathrm{H}$ NMR (400 $\left.\mathrm{MHz}, \mathrm{CDCl}_{3}\right) \delta$ 7.85-7.82 (m, 2H), 7.63-7.60 (m, 1H), 7.52-7.48 (m, 2H) 7.32-7.25 (m, 5H), $6.30(\mathrm{~d}, J=$ $15.7 \mathrm{~Hz}, 1 \mathrm{H}), 6.07$ (dd, J = 16.0, $8.2 \mathrm{~Hz}, 1 \mathrm{H}), 3.89-3.81(\mathrm{~m}, 1 \mathrm{H}), 1.55(\mathrm{~d}, J=7.1 \mathrm{~Hz}, 3 \mathrm{H}) .{ }^{13} \mathrm{C}$ NMR $\left(101 \mathrm{MHz}, \mathrm{CDCl}_{3}\right) \delta 137.0,136.6,135.9,133.8,129.4,129.0,128.8,128.5,126.7$, 122.2, 64.2, 13.6.

\section{2 (3-(phenylsulfonyl)but-1-ene-1,1-diyl)dibenzene (36)}

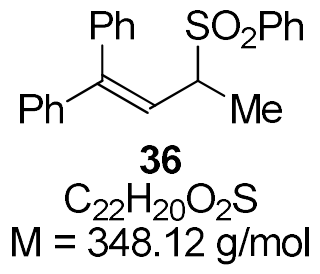

Prepared according to the procedure 1 , by using $22(32.0 \mathrm{mg}, 0.1 \mathrm{mmol}, 1.0$ equiv), $m$ CPBA (34.0 mg, $0.2 \mathrm{mmol}, 2$ equiv) in $\mathrm{DCM}$ at $0{ }^{\circ} \mathrm{C}$ for 4 hours. Purification by column chromatography on silica gel using petroleum ether/ethyl acetate $(10: 1)$ as eluent. The product 36 (23.0 mg, 72\%) was obtained as white solid. M. P. $-91-92{ }^{\circ} \mathrm{C}$. HRMS (ESI): for $\mathrm{C}_{22} \mathrm{H}_{20} \mathrm{O}_{2} \mathrm{~S}^{+}\left[(\mathrm{M}+\mathrm{Na})^{+}\right]$: calculated 371.1082, found 371.1076. ${ }^{1} \mathrm{H}$ NMR $\left(400 \mathrm{MHz}, \mathrm{CDCl}_{3}\right) \delta$ 7.75-7.73 (m, 2H), 7.63-7.59 (m, 1H), 7.49-7.45 (m, 2H), 7.27-7.19 (m, 6H), 7.14-7.12 (m, 2H), 6.57 (dd, $J=7.6,1.5 \mathrm{~Hz}, 2 \mathrm{H}), 6.01(\mathrm{~d}, J=10.9 \mathrm{~Hz}, 1 \mathrm{H}), 3.89-3.81(\mathrm{~m}, 1 \mathrm{H}), 1.58(\mathrm{~d}, J=$ $6.9 \mathrm{~Hz}, 3 \mathrm{H}) .{ }^{13} \mathrm{C}$ NMR $\left(101 \mathrm{MHz}, \mathrm{CDCl}_{3}\right) \delta 147.8,140.7,138.2,133.6,129.1,129.0$ (3C), 128.4(2C), 128.3, 127.8, 127.4, 121.6, 61.0, 13.7.

\section{3 (E)-(2-((4-phenylbut-3-en-2-yl)sulfonyl)propyl)benzene (38)}

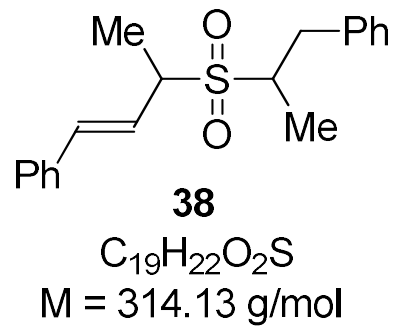

Prepared according to the procedure 1, by using $12(28.21 \mathrm{mg}, 0.1 \mathrm{mmol}, 1.0$ equiv), mCPBA (34.0 mg, $0.2 \mathrm{mmol}, 2$ equiv) in DCM at $0{ }^{\circ} \mathrm{C}$ for 4 hours. Purification by column chromatography on silica gel using petroleum ether/ethyl acetate $(10: 1)$ as eluent. The product 38 (21.0 mg, 68\%, $d r=1: 1)$ was obtained as gummy liquid (mixture of diastereomer). HRMS (ESI): for $\mathrm{C}_{19} \mathrm{H}_{22} \mathrm{O}_{2} \mathrm{~S}^{+}\left[(\mathrm{M}+\mathrm{Na})^{+}\right]$: calculated 337.1238, found 337.1244. ${ }^{1} \mathrm{H}$ NMR (400 $\left.\mathrm{MHz}, \mathrm{CDCl}_{3}\right)$ ס 7.42-7.38 (m, 4H), 7.37-7.34 (m, 3H), 7.33-7.26 (m, 6H), 7.25-7.21 (m, 1H), 7.17-7.12 (m, 3H), 6.57 (dd, $J=43.9,16.0 \mathrm{~Hz}, 2 \mathrm{H}), 6.22$ (dd, $J=25.9,14.9 \mathrm{~Hz}, 2 \mathrm{H}$ ), 3.99$3.92(\mathrm{~m}, 1 \mathrm{H}), 3.88-3.81(\mathrm{~m}, 1 \mathrm{H}), 3.51-3.30(\mathrm{~m}, 4 \mathrm{H}), 2.77-2.66(\mathrm{~m}, 2 \mathrm{H}), 1.62(\mathrm{~d}, J=6.9 \mathrm{~Hz}$, $3 \mathrm{H}), 1.59(\mathrm{~d}, J=6.9 \mathrm{~Hz}, 3 \mathrm{H}) 1.30(\mathrm{~d}, J=6.9 \mathrm{~Hz}, 3 \mathrm{H}), 1.26(\mathrm{~d}, J=6.8 \mathrm{~Hz}, 3 \mathrm{H}) .{ }^{13} \mathrm{C}$ NMR $(101$ $\left.\mathrm{MHz}, \mathrm{CDCl}_{3}\right) \delta 137.1,136.1,135.9,135.6,129.6,129.4,129.0(2 \mathrm{C}), 128.9(2 \mathrm{C}), 128.8(2 \mathrm{C})$, 
$127.3,127.1,126.8(2 \mathrm{C}), 123.4,123.3,59.9,59.3,56.3,56.0,36.5,33.5,13.2,13.0,12.9$, 11.6.

\subsection{Hydrodesulfurization of the synthesized sulfide}

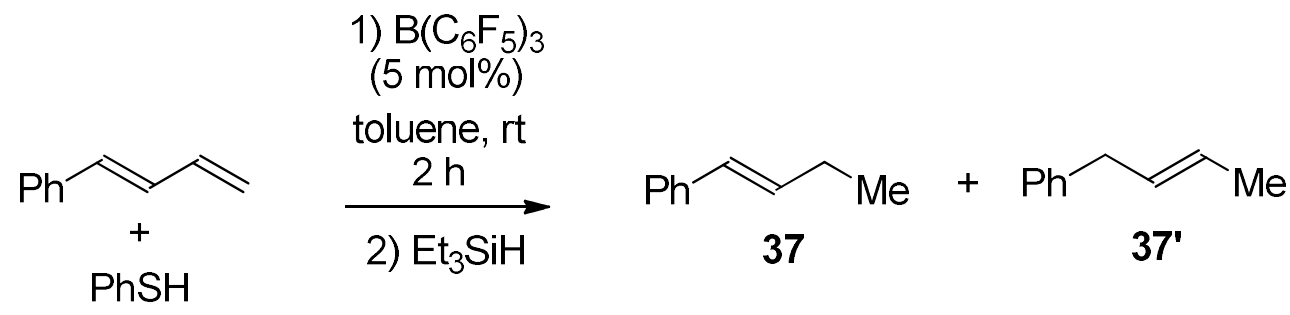

\section{1 (E)-but-1-en-1-ylbenzene and $(E)$-but-2-en-1-ylbenzene $\left(37+37^{\prime}\right)$}

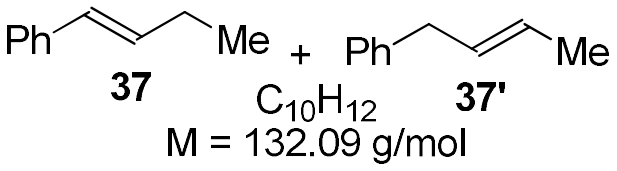

According to GP-A, using 1h (19.0 mg, $0.14 \mathrm{mmol}, 1.2$ equiv), 2a (13.0 mg, $0.12 \mathrm{mmol}, 1.0$ equiv), and tris(pentafluorophenyl)borane $\left(B\left(\mathrm{C}_{6} \mathrm{~F}_{5}\right)_{3}\right)(3.0 \mathrm{mg}, 6.0 \mu \mathrm{mol}, 5.0 \mathrm{~mol} \%)$. Then after two hours triethylsilane $(21.0 \mathrm{mg}, 0.18 \mathrm{mmol}, 1.5$ equiv) was added to the reaction mixture and stirred for additional 30 minutes. ${ }^{[\mathrm{S} 11]}$ Purification by column chromatography on silica gel using petroleum ether as eluent. The product $37+37^{\prime}(15.53 \mathrm{mg}, 98 \%)$ was obtained as colorless liquid. The ratio of $37: \mathbf{3 7}$ from crude is $1.6: 1$, and the isolated ratio is $1: 1.4$.

${ }^{1} \mathrm{H}$ NMR $\left(400 \mathrm{MHz}, \mathrm{CDCl}_{3}\right)$ ठ 7.35-7.32 (m, 1H), $7.28(\mathrm{dd}, J=8.5,6.9 \mathrm{~Hz}, 3 \mathrm{H}), 7.20-7.16(\mathrm{~m}$, $4 \mathrm{H}), 6.39-6.34(\mathrm{~m}, 1 \mathrm{H}), 6.29-6.22(\mathrm{~m}, 1 \mathrm{H}), 5.62-5.46(\mathrm{~m}, 2 \mathrm{H}), 3.31(\mathrm{~d}, J=6.2 \mathrm{~Hz}, 2 \mathrm{H})$, 2.26-2.19 (m, 2H), $1.68(\mathrm{dd}, J=6.0,1.5 \mathrm{~Hz}, 3 \mathrm{H}), 1.08(\mathrm{t}, J=7.4 \mathrm{~Hz}, 2 \mathrm{H}) .{ }^{13} \mathrm{C}$ NMR (101 $\left.\mathrm{MHz}, \mathrm{CDCl}_{3}\right) \delta 132.8,130.2,128.9,128.6,128.5,126.9,126.5,126.0(2 \mathrm{C}), 39.2,26.2,18.1$, 13.8. 


\subsection{Deuterium labelling}

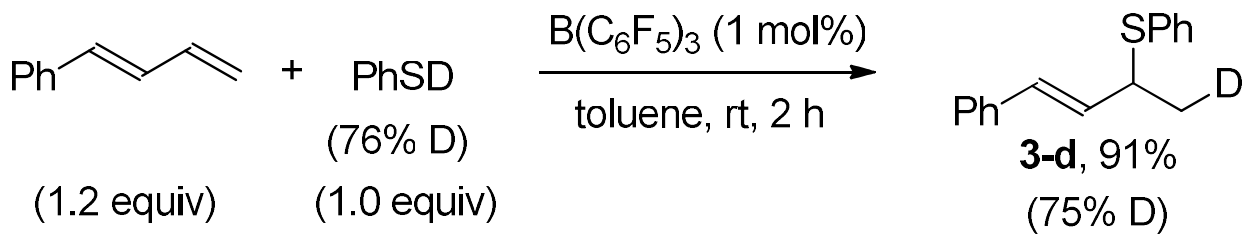

According to GP-A, using 1a (91.0 mg, $0.69 \mathrm{mmol}, 1.2$ equiv), 2a-d (64.0 mg, $0.58 \mathrm{mmol}, 1.0$ equiv), and tris(pentafluorophenyl)borane $\left(\mathrm{B}_{(}\left(\mathrm{C}_{6} \mathrm{~F}_{5}\right)_{3}\right)(3.0 \mathrm{mg}, 5.8 \mu \mathrm{mol}, 1.0 \mathrm{~mol} \%)$. Purification by column chromatography on silica gel using petroleum ether as eluent. The product 3-d (127.0 $\mathrm{mg}$, yield $91 \%$,) was obtained as colorless liquid. $75 \%$ deuterium labelling has been confirmed by the ${ }^{1} \mathrm{H}$ NMR.

\section{1 (E)-phenyl(4-phenylbut-3-en-2-yl)sulfane- $d_{1}(3-d)$}

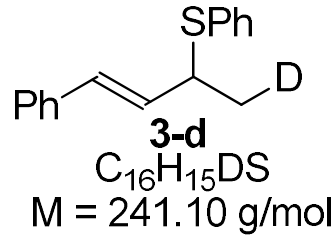

D-MASS (ESI): for $\mathrm{C}_{16} \mathrm{H}_{15} \mathrm{DS}^{+}\left[(\mathrm{M}+\mathrm{H})^{+}\right]$: calculated 242.11, found 242.10. ${ }^{1} \mathrm{H}$ NMR (400 MHz, $\left.\mathrm{CDCl}_{3}\right)$ ठ 7.44-7.42 (m, 2H), 7.29-7.27 (m, 5H), 7.25-7.19 (m, 3H), 6.24-6.13 (m, 2H), 3.93$3.87(\mathrm{~m}, 1 \mathrm{H}), 1.50-1.45(\mathrm{~m}, 2.25 \mathrm{H}) .{ }^{13} \mathrm{C}$ NMR $\left(101 \mathrm{MHz}, \mathrm{CDCl}_{3}\right) \delta 137.0,134.6,133.5$, 131.7, 130.0, 128.8, 128.6, 127.5 (2C), 126.4, 46.6, $20.7\left(20.7,20.5,20.3\right.$ for $\left.\mathrm{CH}_{2} \mathrm{D}\right) .{ }^{13} \mathrm{C}$ DEPT-135 NMR (101 MHz, $\left.\mathrm{CDCl}_{3}\right) \delta 137.0,134.6,133.5,131.7,130.0,128.8,128.6$, 127.5(2C), 126.4, 46.5, 20.7 (20.6, 20.4, 20.2 for $\left.\mathrm{CH}_{2} \mathbf{D}\right)$. 


\subsection{Time-Dependent ${ }^{1} \mathrm{H}-\mathrm{NMR}$ Measurement of Model Reaction}

According to GP-A, using 1a (91.0 mg, 0.69 mmol, 1.2 equiv), 2a (64.0 mg, $0.58 \mathrm{mmol}, 1.0$ equiv), and tris(pentafluorophenyl)borane $\left(B\left(\mathrm{C}_{6} \mathrm{~F}_{5}\right)_{3}\right)(3.0 \mathrm{mg}, 5.8 \mu \mathrm{mol}, 1.0 \mathrm{~mol} \%)$ in toluene$d_{8}$. Complete reaction performed inside the glove box and the NMR samples prepared inside the glove box by taking aliquots from reaction mixture at every 30 minutes interval time. First NMR recorded for the initial aliquot which has been taken just after reaction setup. In similar manner all the NMR has been recorded.
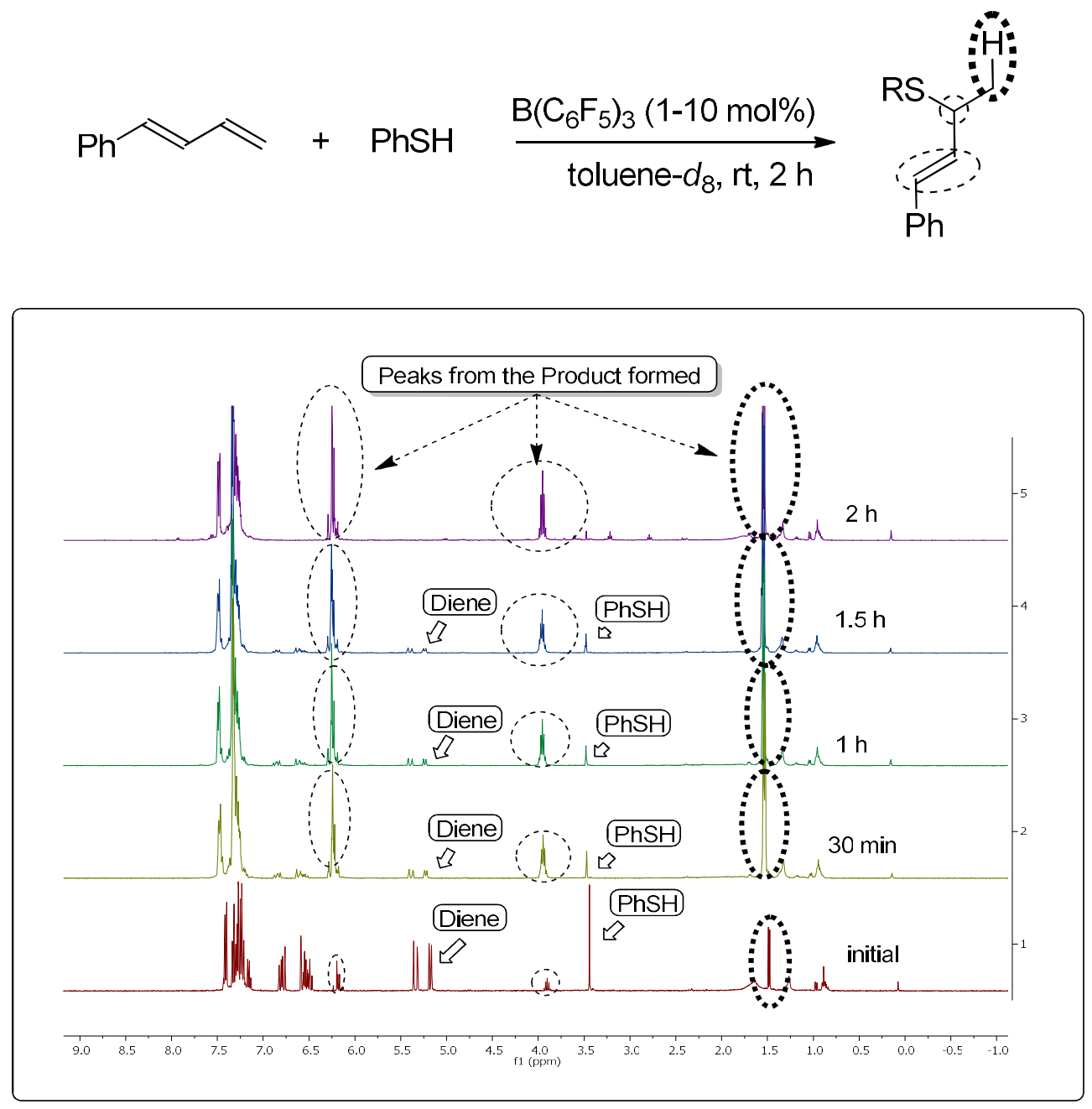

${ }^{1} \mathrm{H}$ NMR $\left(400 \mathrm{MHz}, \mathrm{CDCl}_{3}\right)$ 


\section{0 ${ }^{11} B$ NMR for the detection of $B\left(C_{6} F_{5}\right)_{3}$ and Thiophenol Adduct formation}

The Boron NMR has been recorded for the detection of the Lewis acid and thiophenol adduct formation upto $-30{ }^{\circ} \mathrm{C}$. As the DFT calculations have shown the stability of this adduct at lower temperature, proved by the ${ }^{11} \mathrm{~B} N M R$, the Chemical shift value for Boron shifted towards upfield region.

$\mathrm{B}\left(\mathrm{C}_{6} \mathrm{~F}_{5}\right)_{3}$ and Thiophenol at $-30{ }^{\circ} \mathrm{C},{ }^{11} \mathrm{~B}$ NMR $\left(128 \mathrm{MHz}\right.$, TOLUENE- $\left.D_{8}\right) \delta 1.51$.

$\mathrm{B}\left(\mathrm{C}_{6} \mathrm{~F}_{5}\right)_{3}$ and Thiophenol at $18{ }^{\circ} \mathrm{C},{ }^{11} \mathrm{~B}$ NMR $\left(128 \mathrm{MHz}\right.$, TOLUENE- $\left.D_{8}\right) \delta 21.73$.

$\mathrm{B}\left(\mathrm{C}_{6} \mathrm{~F}_{5}\right)_{3}$ at $18{ }^{\circ} \mathrm{C},{ }^{11} \mathrm{~B}$ NMR $\left(128 \mathrm{MHz}\right.$, TOLUENE- $\left.D_{8}\right) \delta 58.51$

군

$\mathrm{B}\left(\mathrm{C}_{6} \mathrm{~F}_{5}\right)_{3}+\mathrm{PhSH}$ in Toluene- $d_{8}$ at $-30^{\circ} \mathrm{C}$
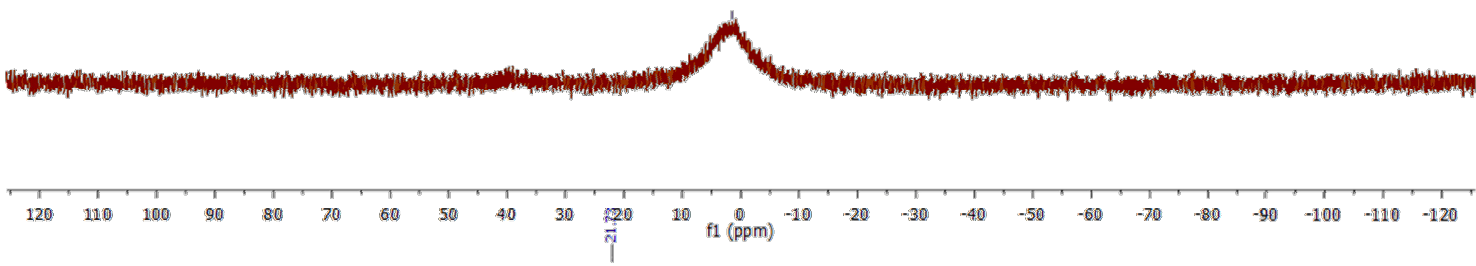

$\mathrm{B}\left(\mathrm{C}_{6} \mathrm{~F}_{5}\right)_{3}+\mathrm{PhSH}$ in Toluene- $d_{8}$ at $18^{\circ} \mathrm{C}$
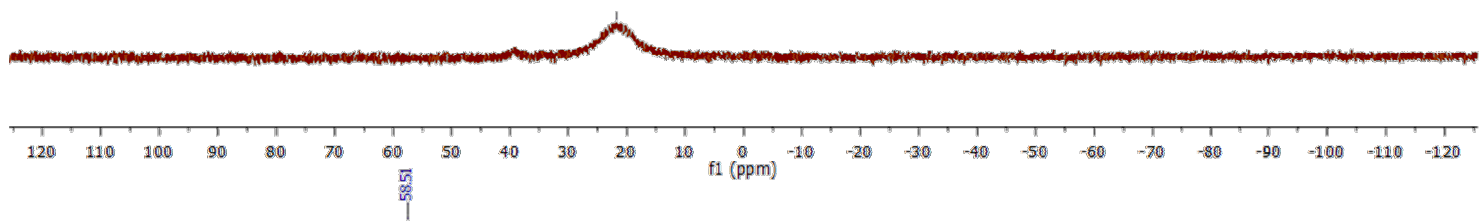

$\mathrm{B}\left(\mathrm{C}_{6} \mathrm{~F}_{5}\right)_{3}$ in Toluene- $d_{8}$ at $18^{\circ} \mathrm{C}$

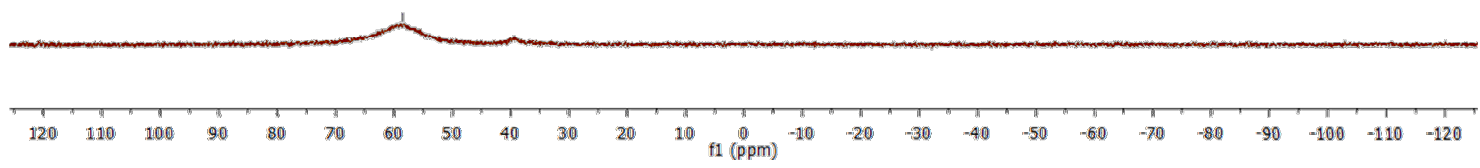

${ }^{11}$ B NMR (128 MHz TOLUENE- D $)$ 


\subsection{NMR Spectra}

(E)-phenyl(4-phenylbut-3-en-2-yl)sulfane (3)

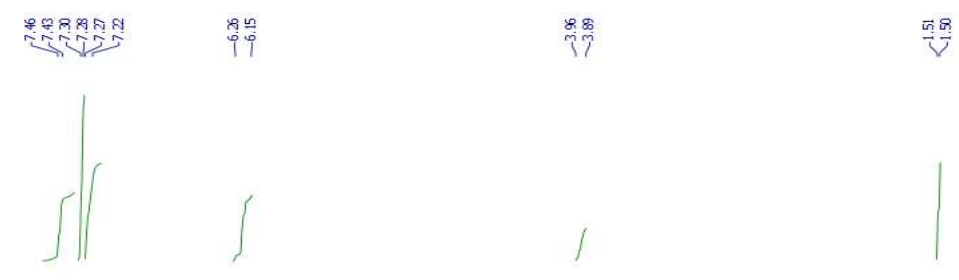

${ }^{1} \mathrm{H}$ NMR $\left(400 \mathrm{MHz}, \mathrm{CDCl}_{3}\right)$

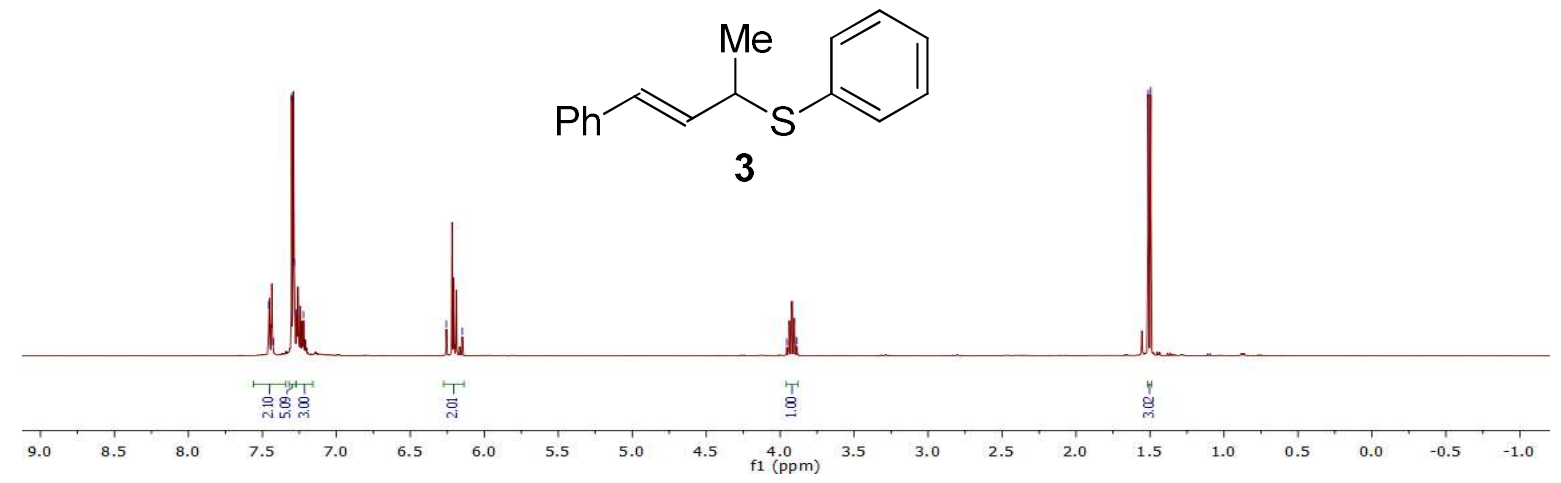

${ }^{13} \mathrm{C}$ NMR $\left(101 \mathrm{MHz}, \mathrm{CDCl}_{3}\right)$

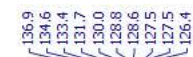

,
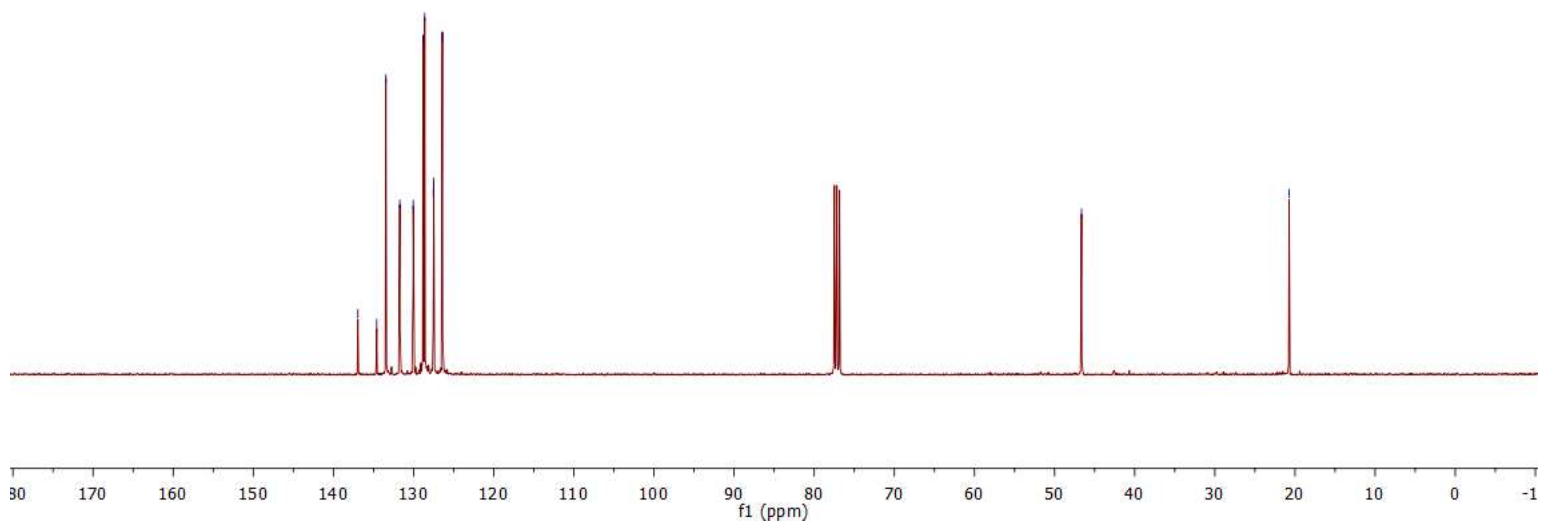
(E)-(4-chlorophenyl)(4-phenylbut-3-en-2-yl)sulfane (4)

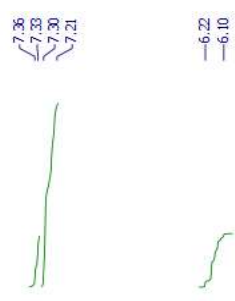

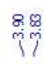

$\stackrel{2}{-i f}$

${ }^{1} \mathrm{H}$ NMR $\left(400 \mathrm{MHz}, \mathrm{CDCl}_{3}\right)$
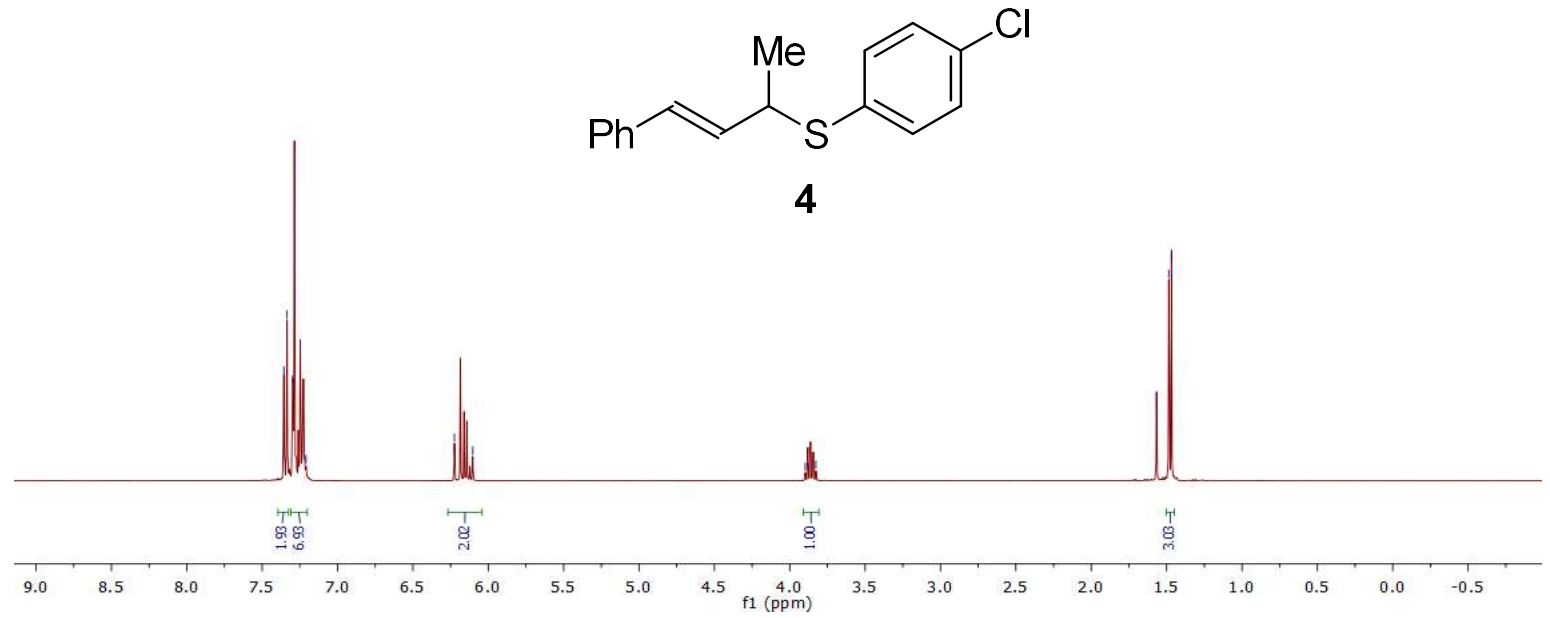

${ }^{13} \mathrm{C}$ NMR $\left(101 \mathrm{MHz}, \mathrm{CDCl}_{3}\right)$

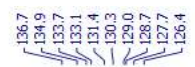

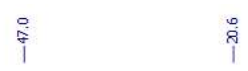

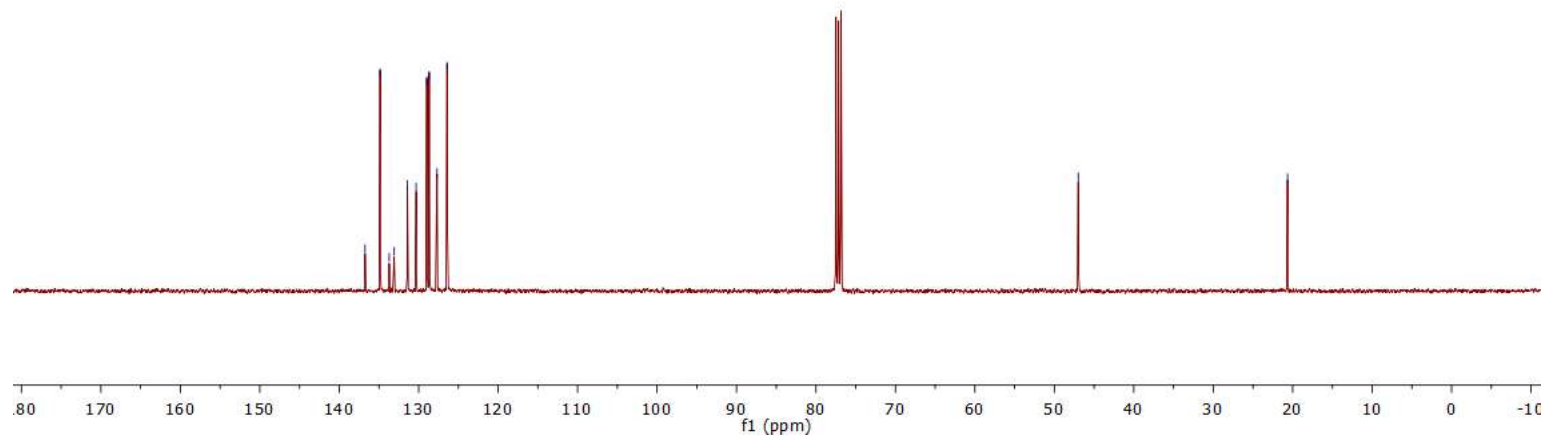

S36 
(E)-(4-methoxyphenyl)(4-phenylbut-3-en-2-yl)sulfane (5)
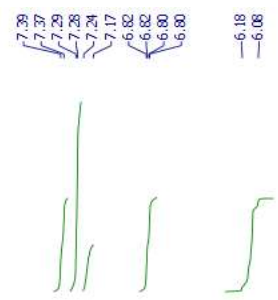

${ }^{1} \mathrm{H} \mathrm{NMR}\left(400 \mathrm{MHz}, \mathrm{CDCl}_{3}\right)$
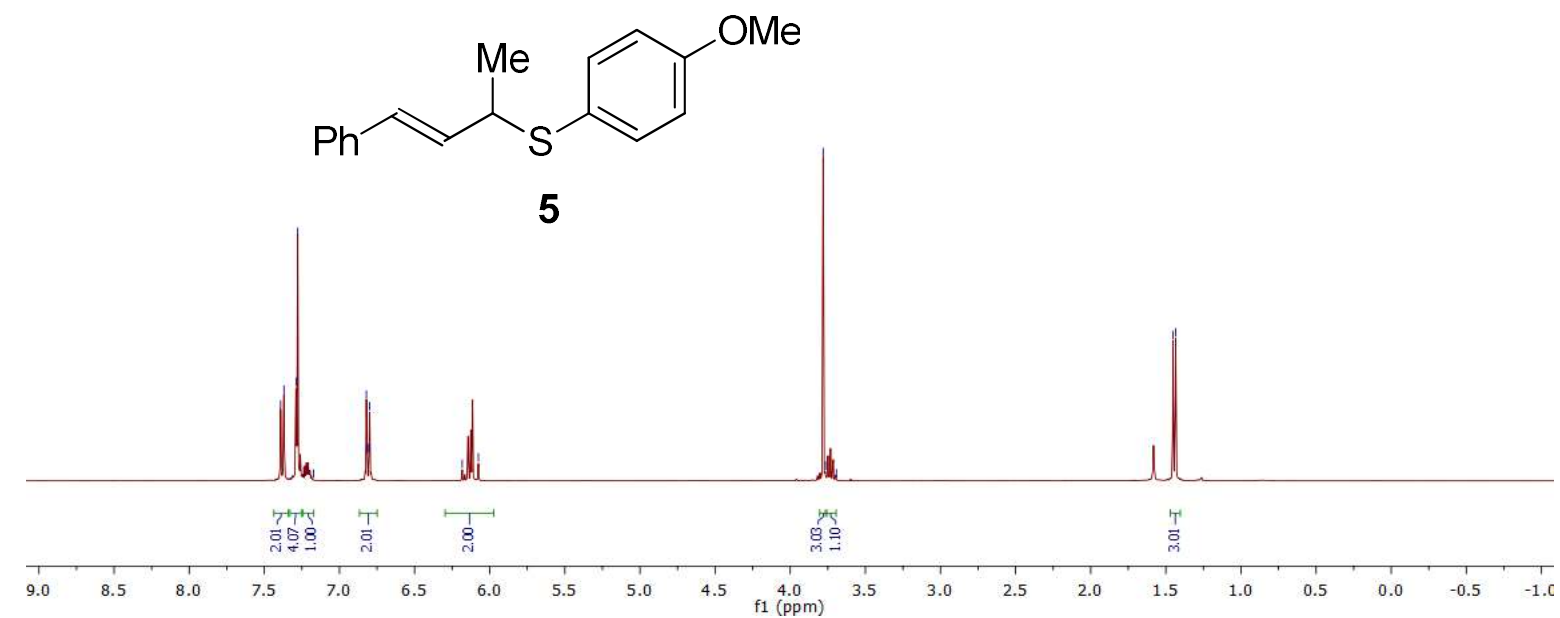

${ }^{13} \mathrm{C}$ NMR $\left(101 \mathrm{MHz}, \mathrm{CDCl}_{3}\right)$

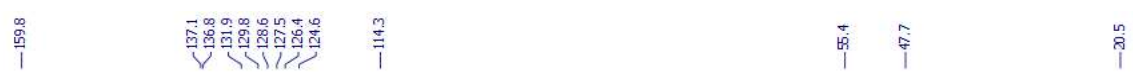
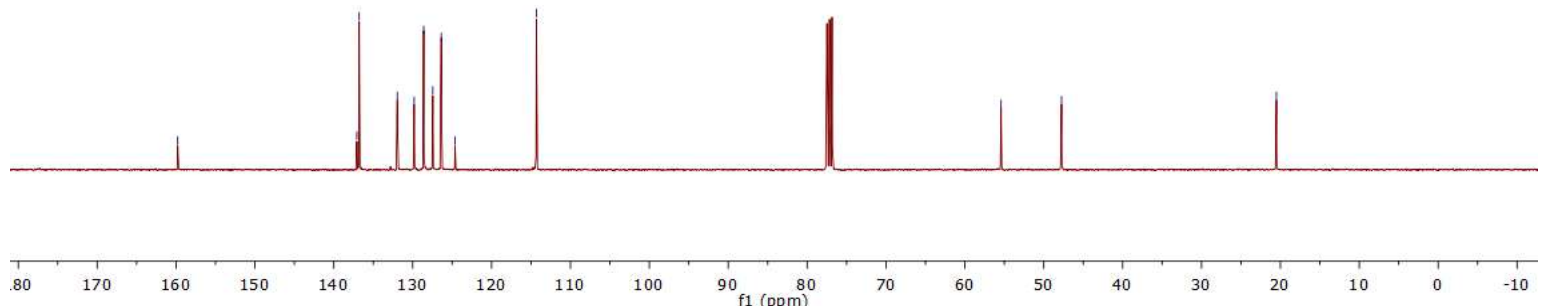
(E)-naphthalen-1-yl(4-phenylbut-3-en-2-yl)sulfane (6)
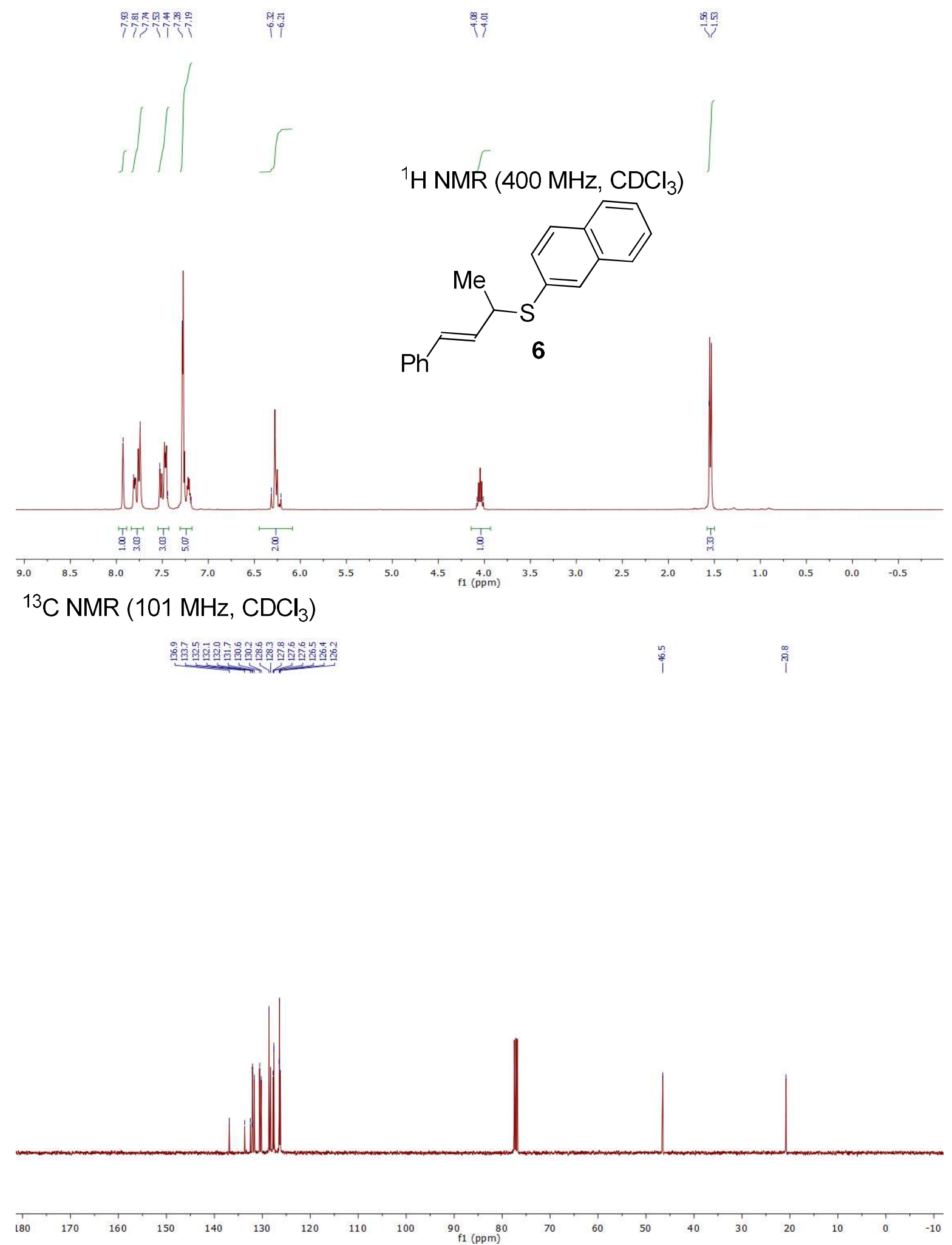
(E)-benzyl(4-phenylbut-3-en-2-yl)sulfane (7)
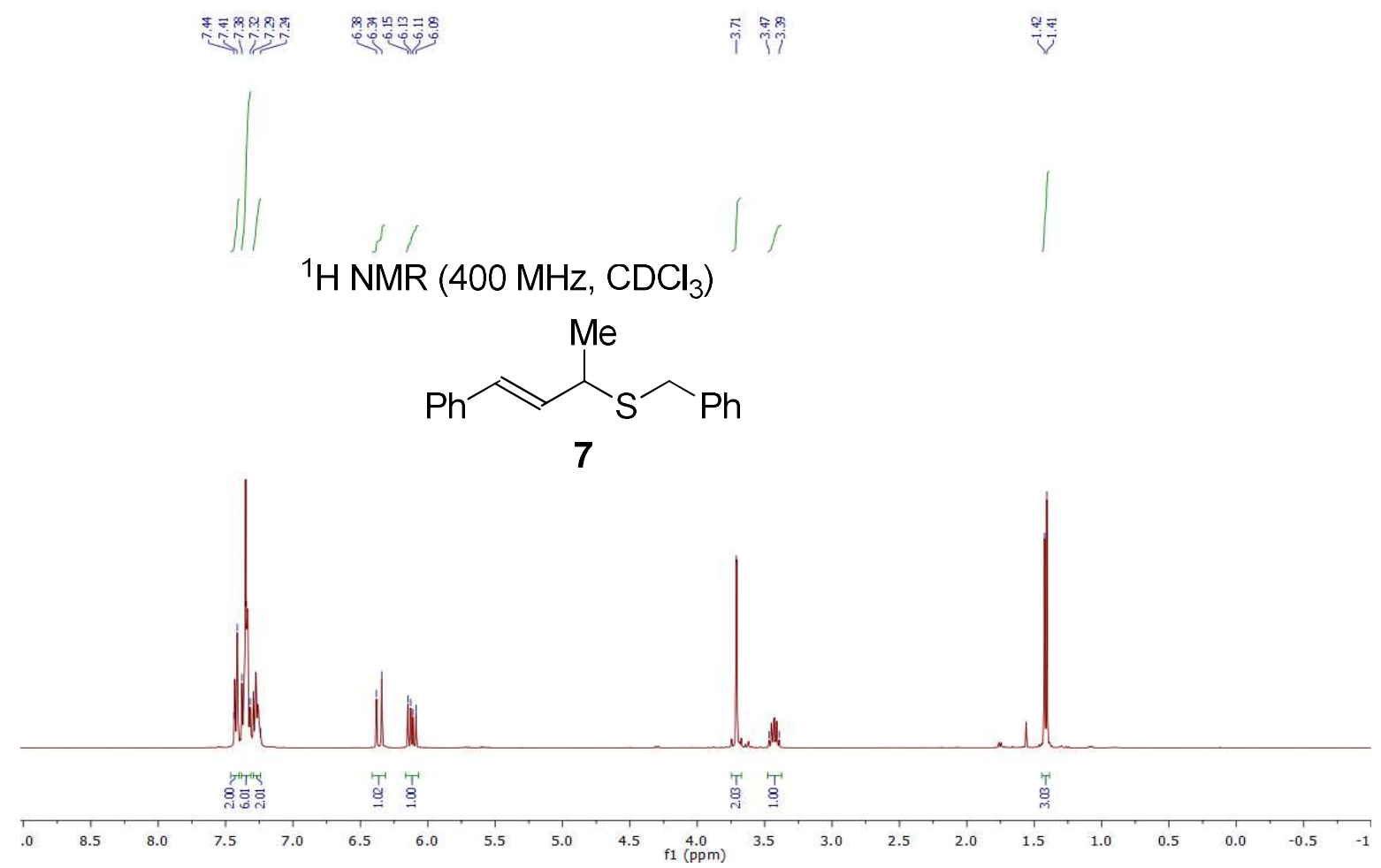

${ }^{13} \mathrm{C}$ NMR $\left(101 \mathrm{MHz}, \mathrm{CDCl}_{3}\right)$

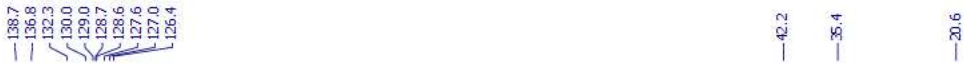
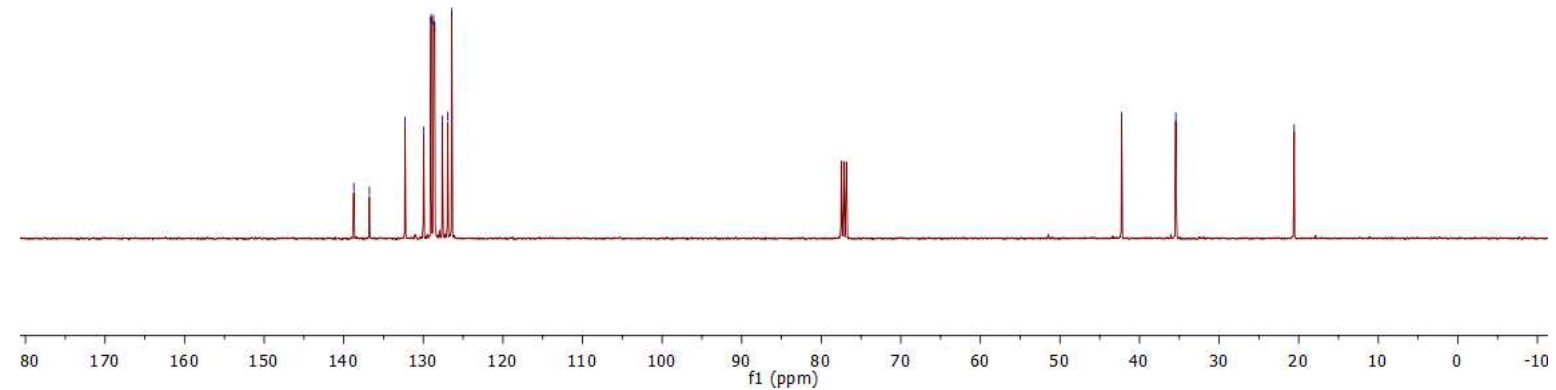
(E)-2-(((4-phenylbut-3-en-2-yl)thio)methyl)furan (8)
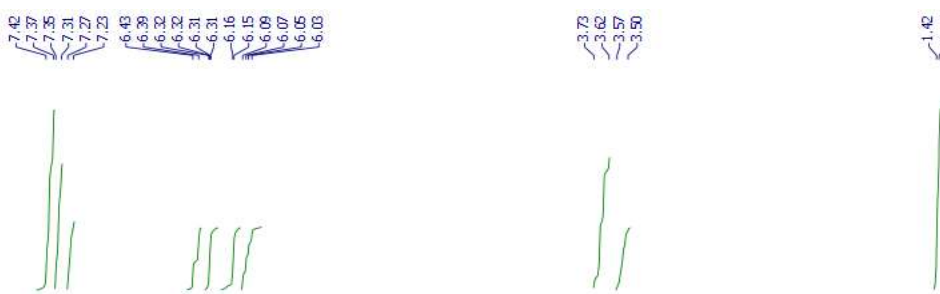

${ }^{1} \mathrm{H}$ NMR $\left(400 \mathrm{MHz}, \mathrm{CDCl}_{3}\right)$<smiles>CC(C=Cc1ccccc1)SCc1ccco1</smiles>

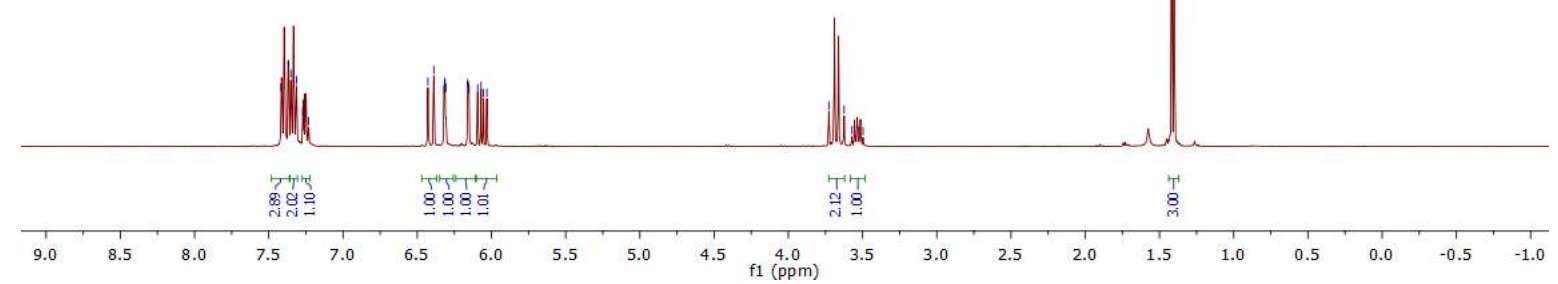

${ }^{13} \mathrm{C}$ NMR $\left(101 \mathrm{MHz}^{\mathrm{CDCl}} 3\right)$

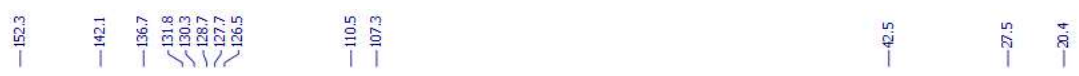
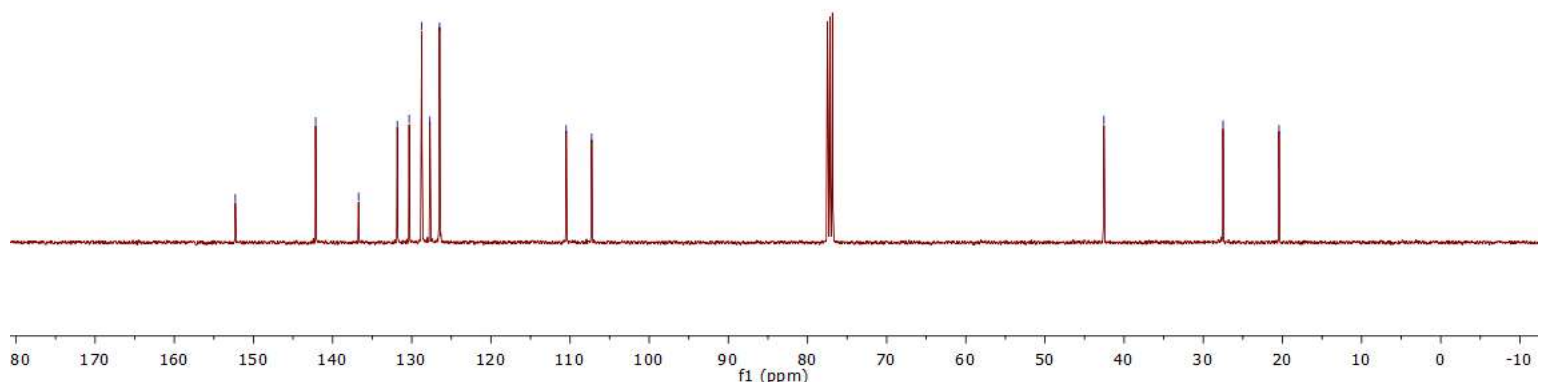
(E)-isopentyl(4-phenylbut-3-en-2-yl)sulfane (9)

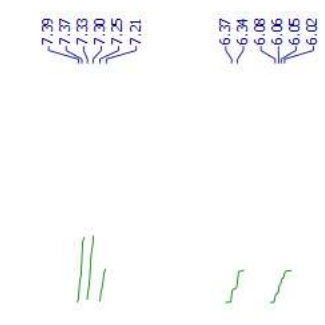

דim

${ }^{1} \mathrm{H}$ NMR $\left(400 \mathrm{MHz}, \mathrm{CDCl}_{3}\right)$
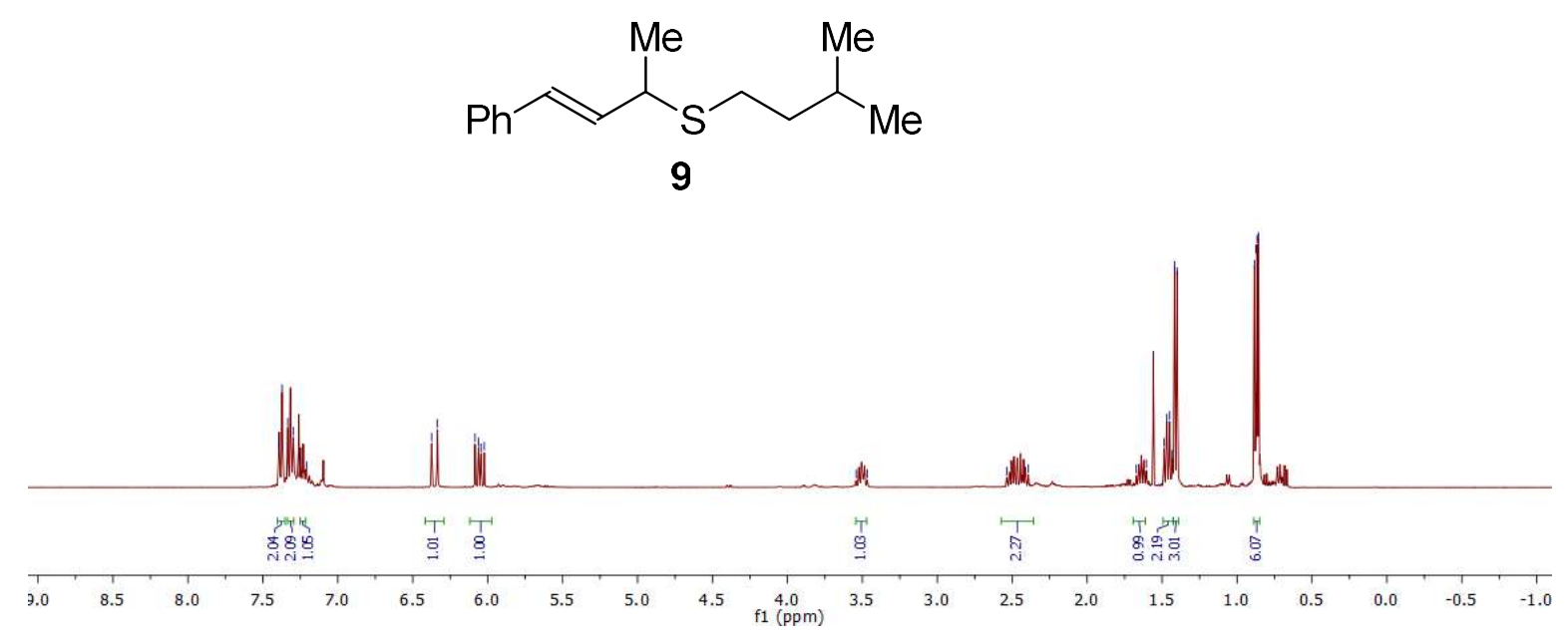

${ }^{13} \mathrm{C}$ NMR $\left(101 \mathrm{MHz}, \mathrm{CDCl}_{3}\right)$

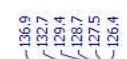

$1<411$

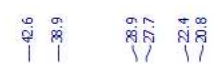
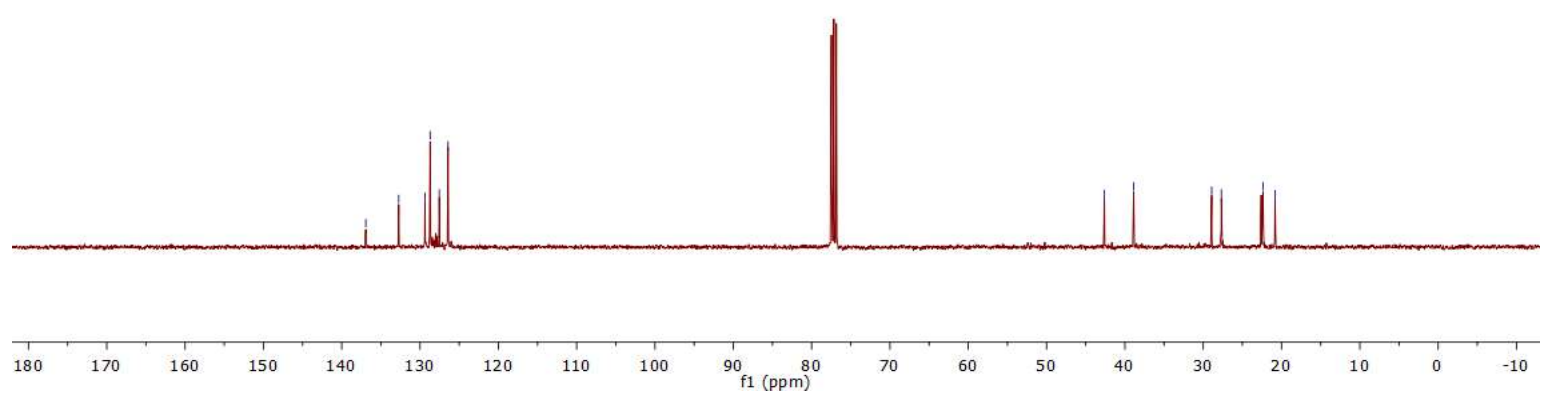
(E)-cyclohexyl(4-phenylbut-3-en-2-yl)sulfane (10)

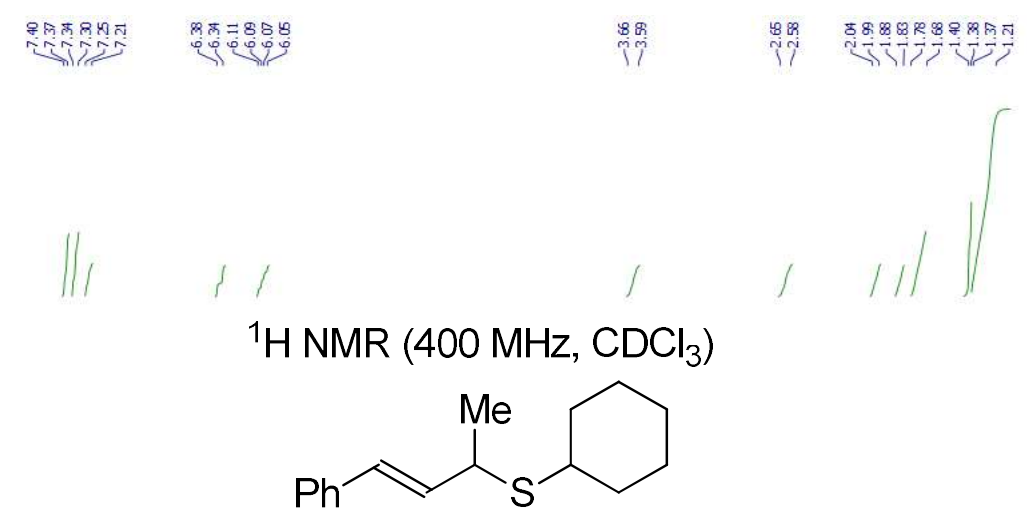

10

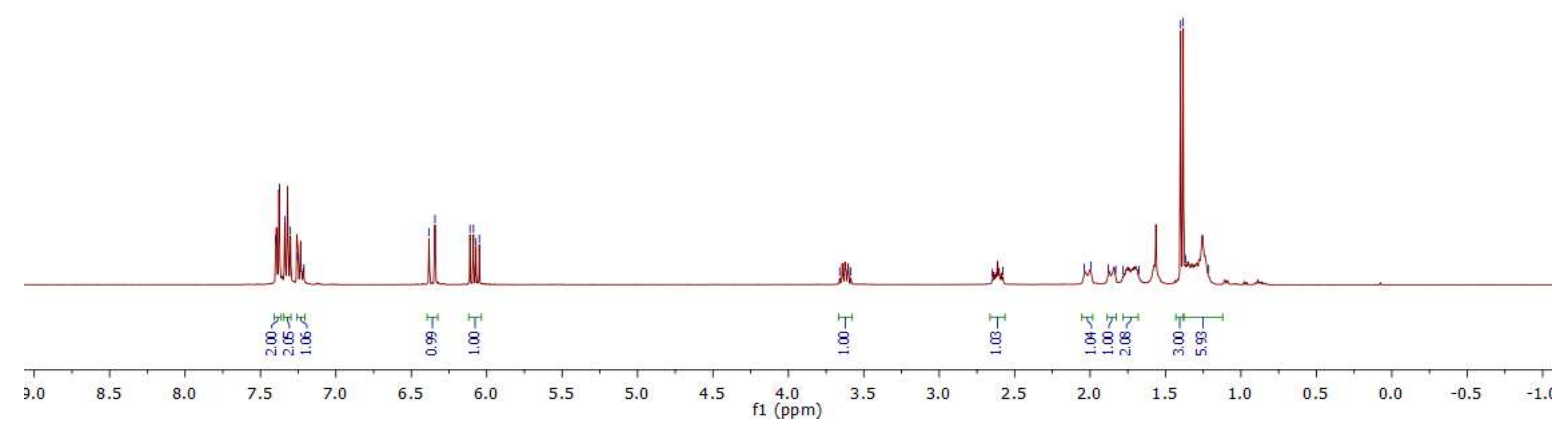

${ }^{13} \mathrm{C}$ NMR $\left(101 \mathrm{MHz}, \mathrm{CDCl}_{3}\right)$

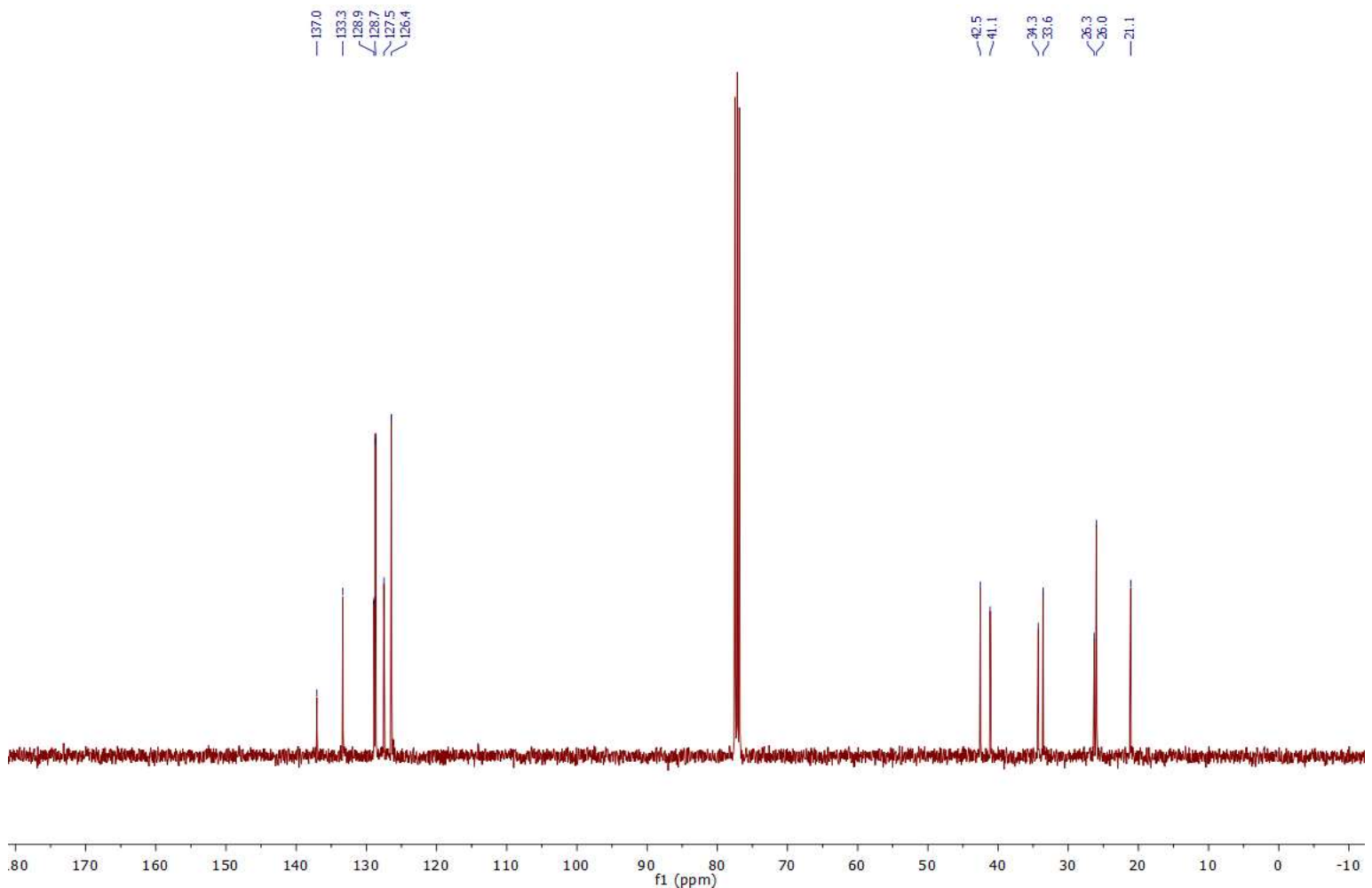


(E)-benzyl 3-((4-phenylbut-3-en-2-yl)thio)propanoate (11)
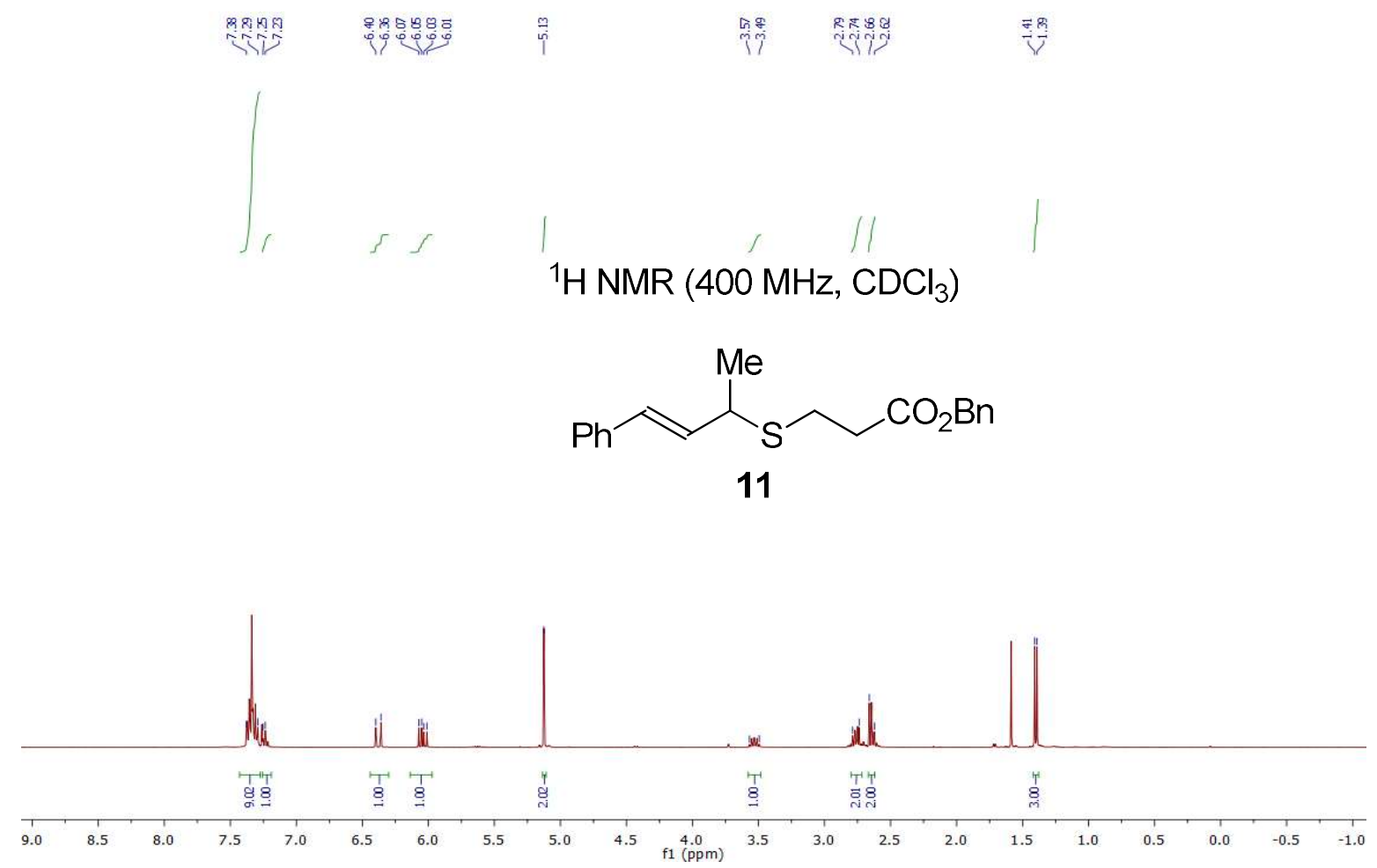

${ }^{13} \mathrm{C}$ NMR (101 MHz, $\left.\mathrm{CDCl}_{3}\right)$
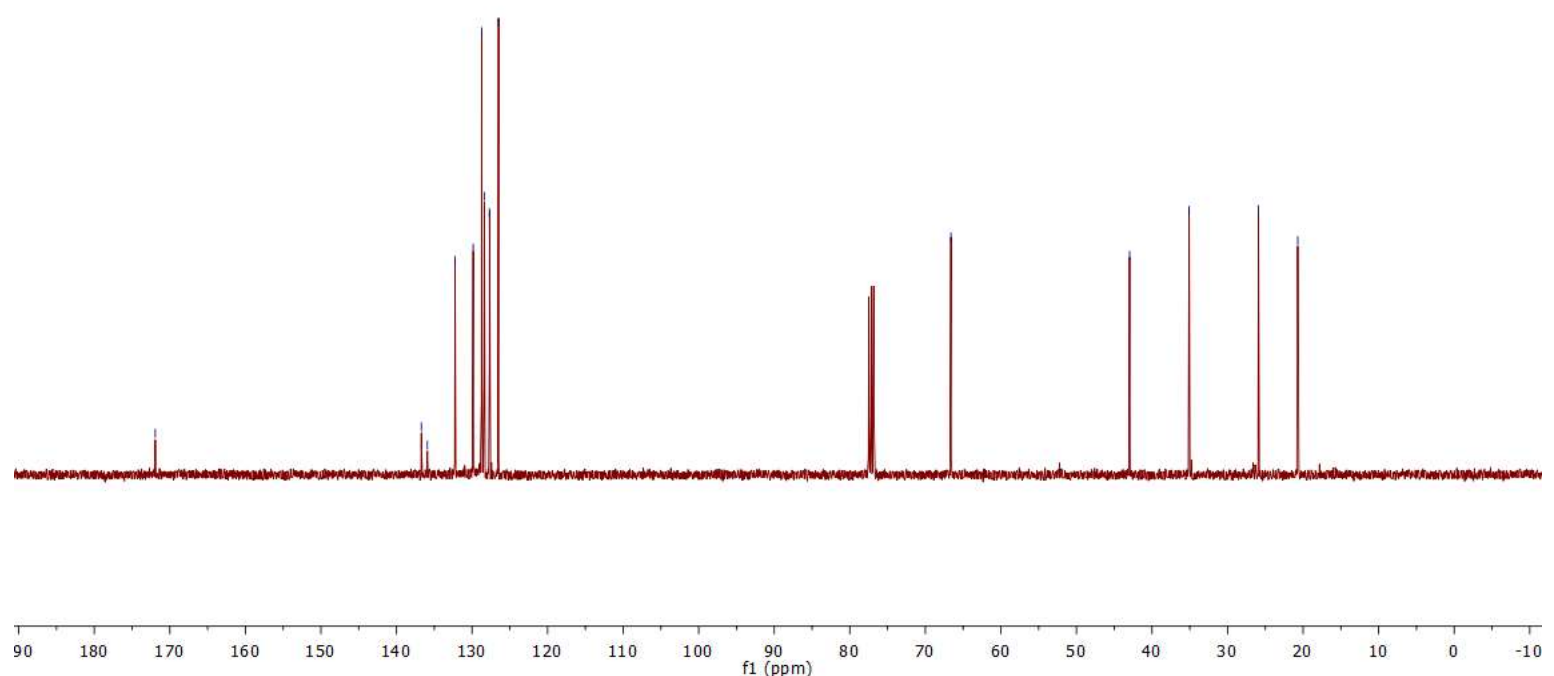
(E)-(4-phenylbut-3-en-2-yl)(1-phenylpropan-2-yl)sulfane (12)

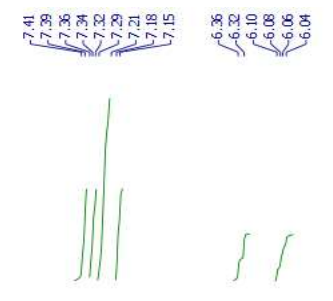

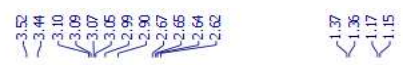

${ }^{1} \mathrm{H} \mathrm{NMR}\left(400 \mathrm{MHz}, \mathrm{CDCl}_{3}\right)$<smiles>CC(C=Cc1ccccc1)SC(C)Cc1ccccc1</smiles>

12

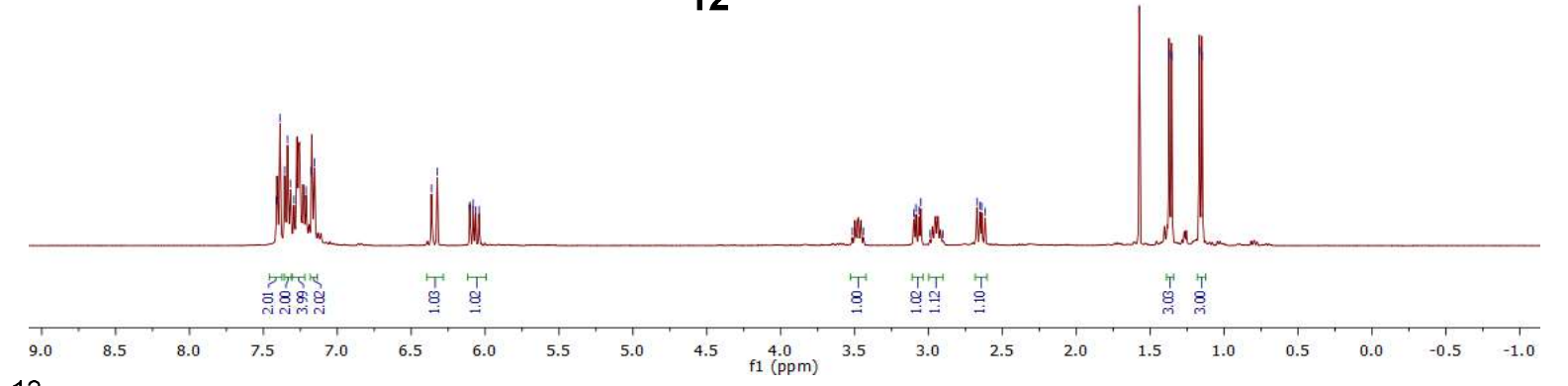

${ }^{13} \mathrm{C}$ NMR (101 $\left.\mathrm{MHz}, \mathrm{CDCl}_{3}\right)$

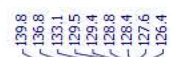

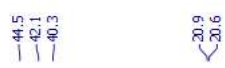
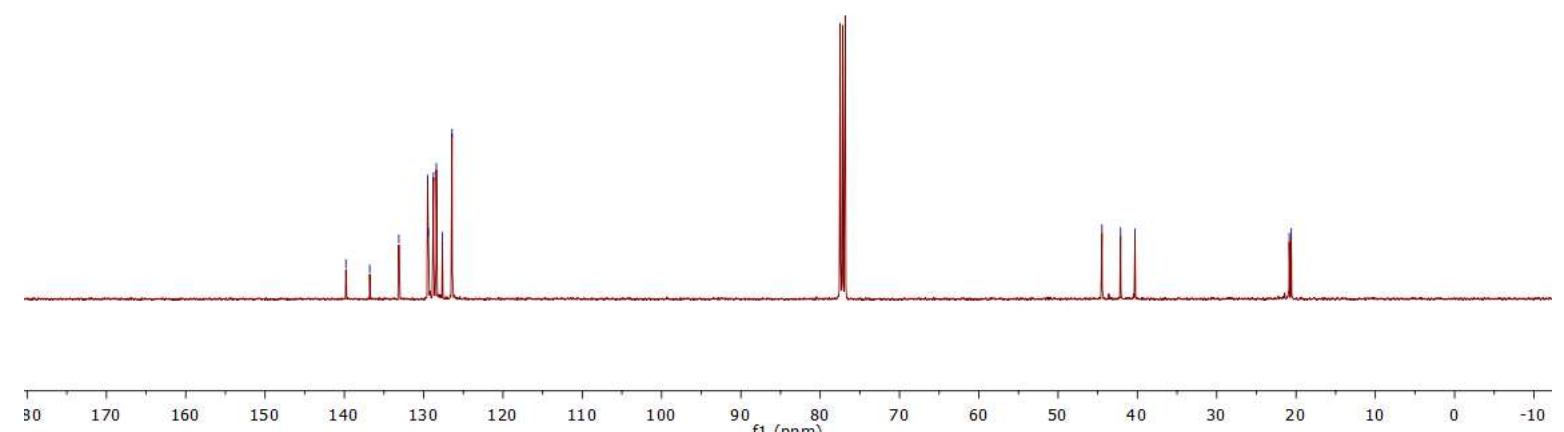

${ }_{\mathrm{f} 1(\mathrm{ppm})}^{80}$ 


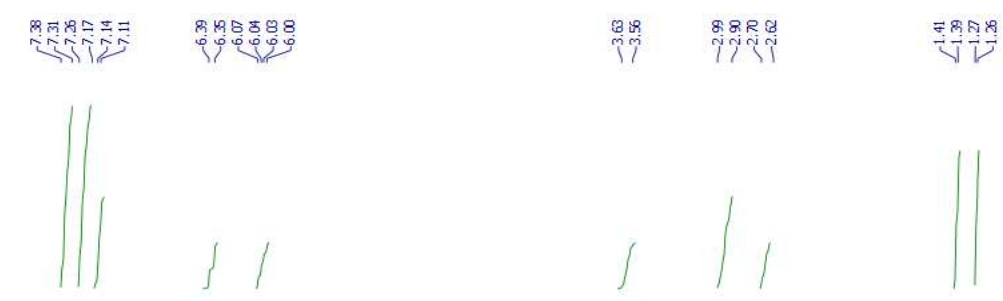

${ }^{1} \mathrm{H}$ NMR (400 MHz, $\mathrm{CDCl}_{3}$ )
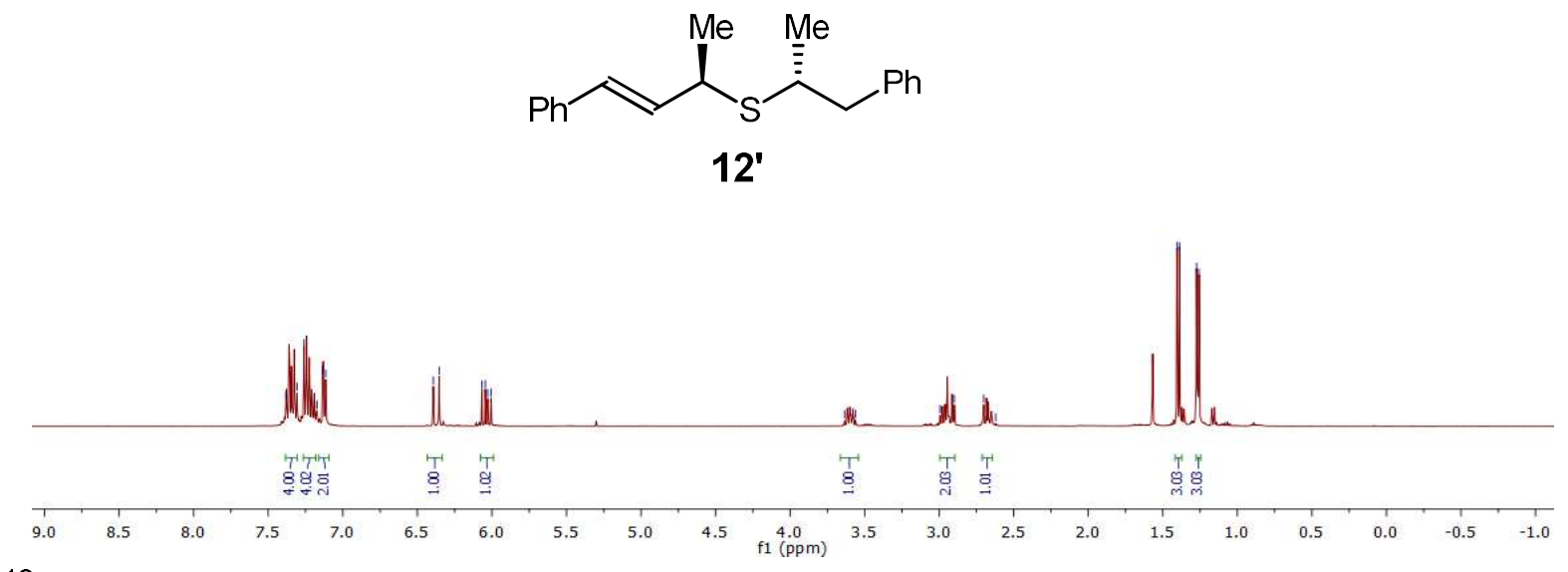

${ }^{13} \mathrm{C}$ NMR (101 MHz, $\mathrm{CDCl}_{3}$ )
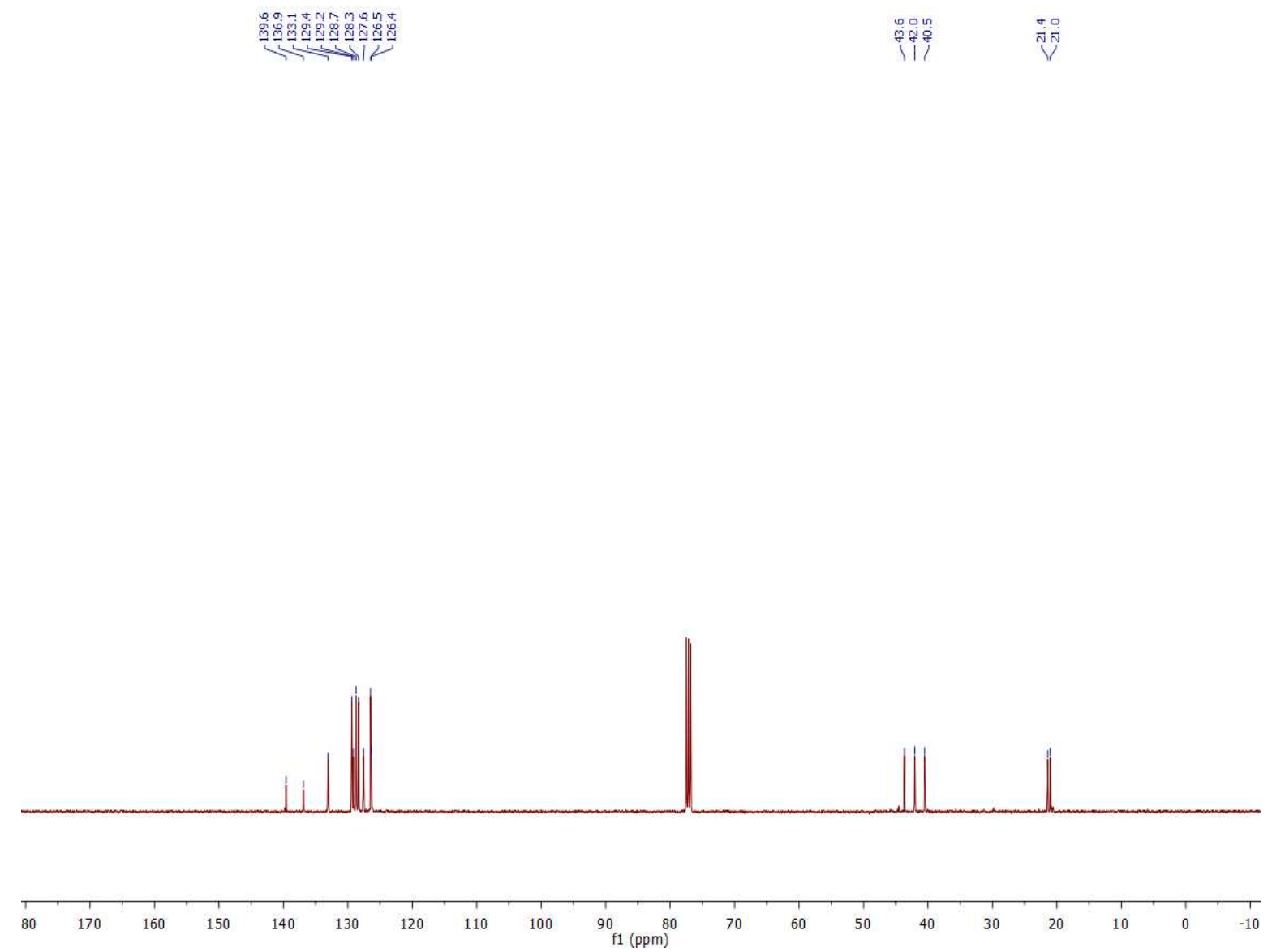
(E)-3-((4-phenylbut-3-en-2-yl)thio)propane-1-thiol (13)

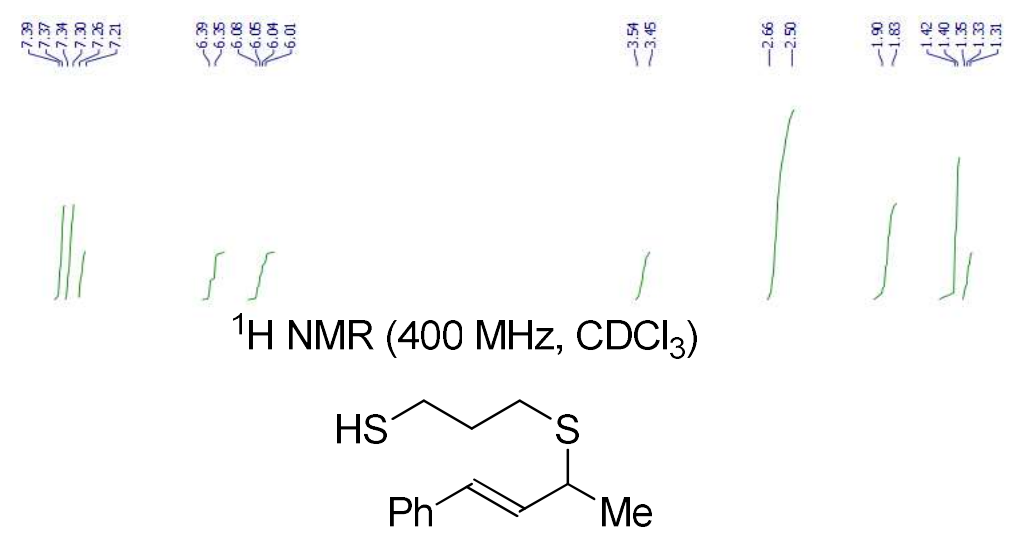

13

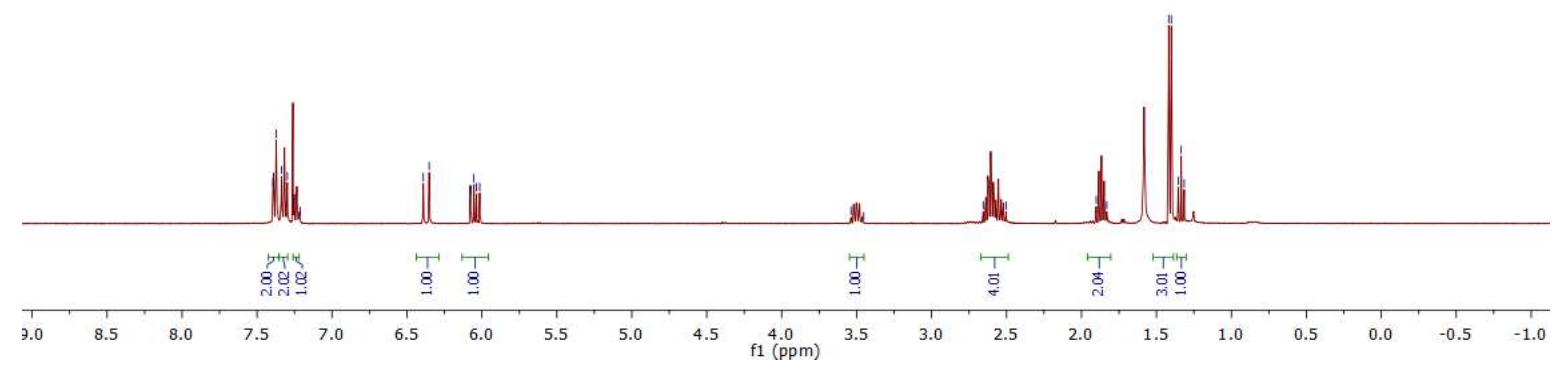

${ }^{13} \mathrm{C}$ NMR $\left(101 \mathrm{MHz}, \mathrm{CDCl}_{3}\right)$

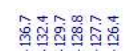

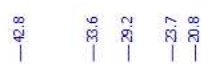
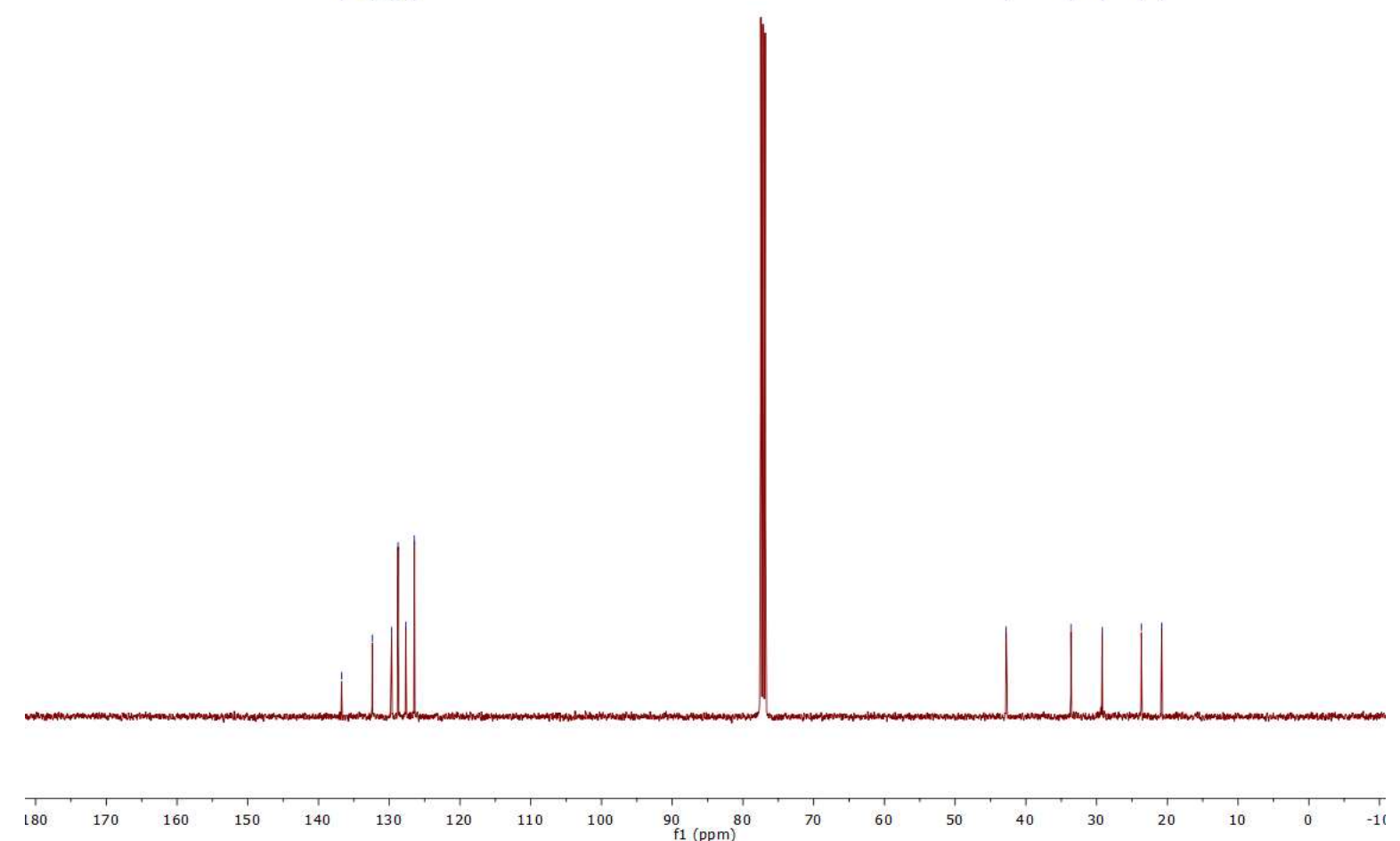
((E)-4-phenylbut-3-en-2-yl)(3-(((E)-5-phenylpent-4-en-2-yl)thio)propyl)sulfane (13')

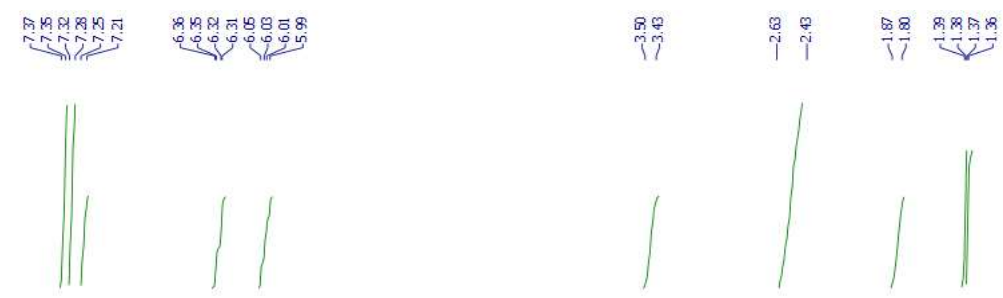

${ }^{1} \mathrm{H}$ NMR (400 MHz, $\left.\mathrm{CDCl}_{3}\right)$<smiles>CC(/C=C/c1ccccc1)SCC/C=C\c1ccccc1</smiles>

$13^{\prime}$

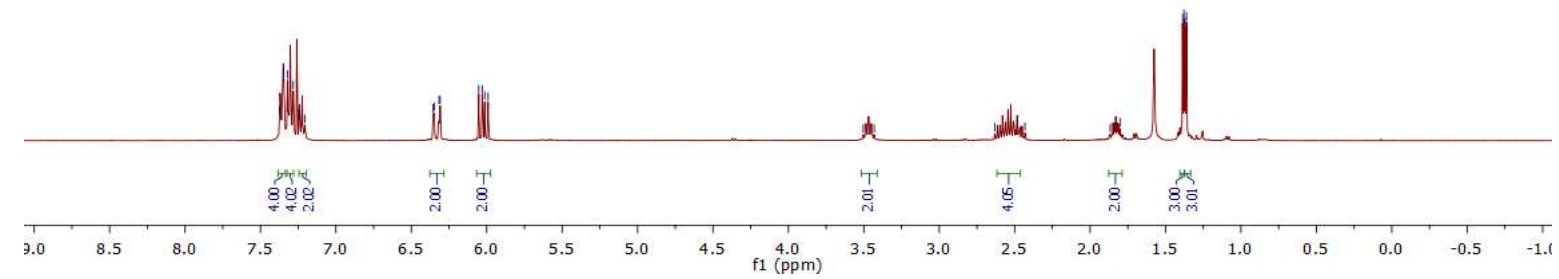

${ }^{13} \mathrm{C}$ NMR $\left(101 \mathrm{MHz}, \mathrm{CDCl}_{3}\right)$

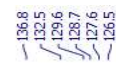

iิ

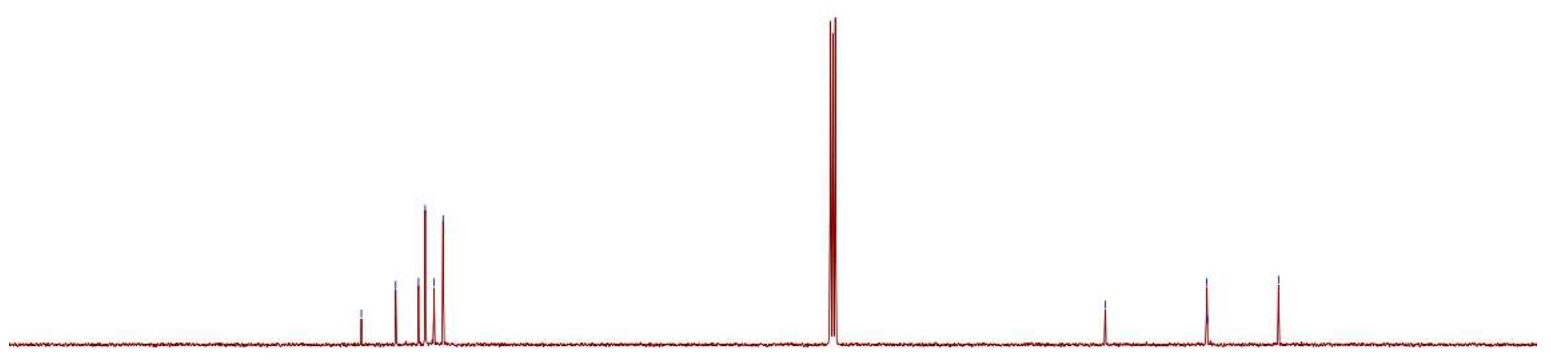

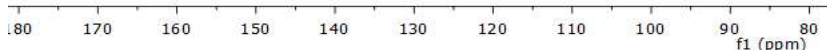


(E)-phenyl(4-(p-tolyl)but-3-en-2-yl)sulfane (14)
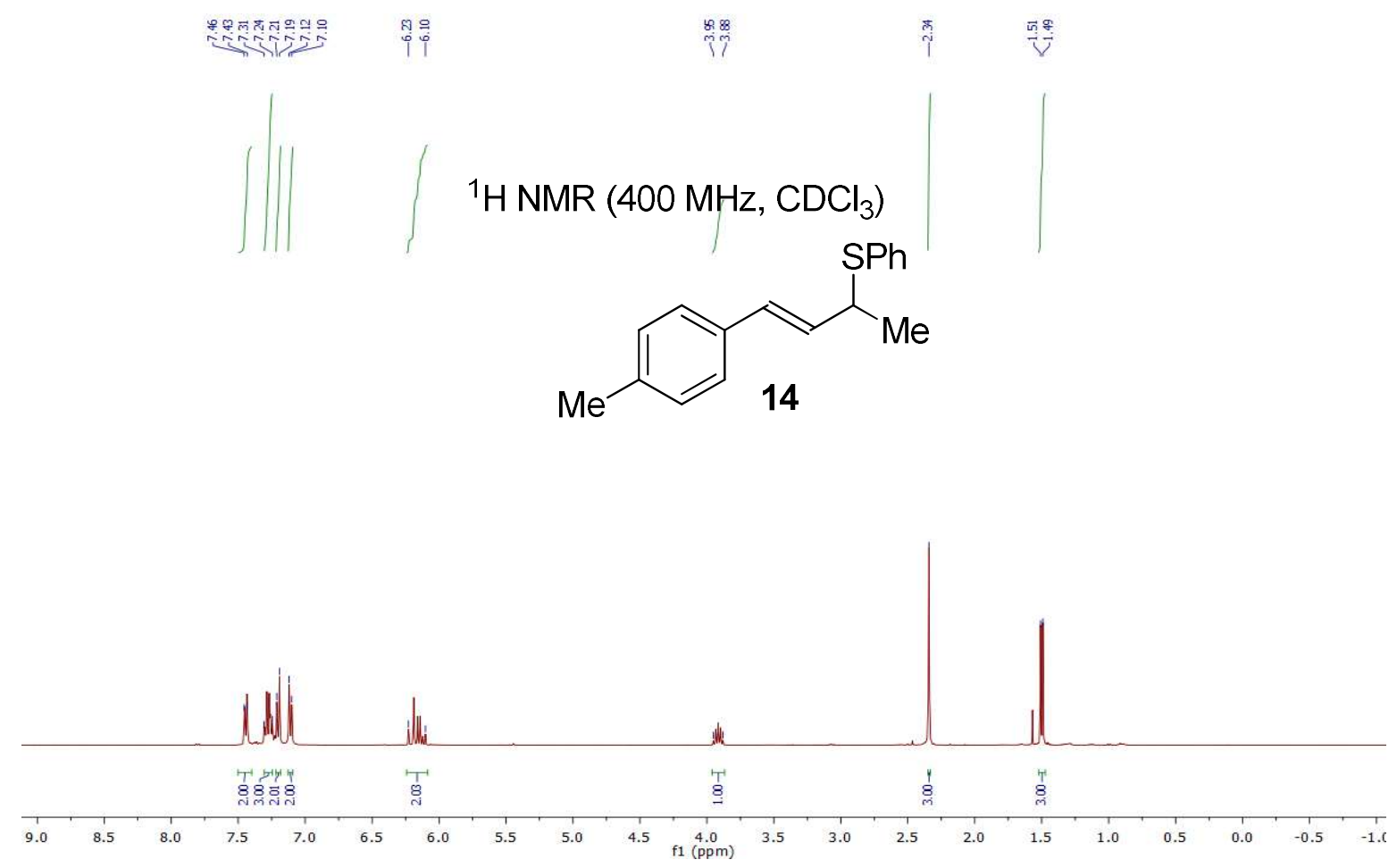

${ }^{13} \mathrm{C}$ NMR $\left(101 \mathrm{MHz}, \mathrm{CDCl}_{3}\right)$

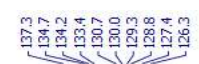

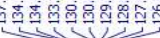

ind
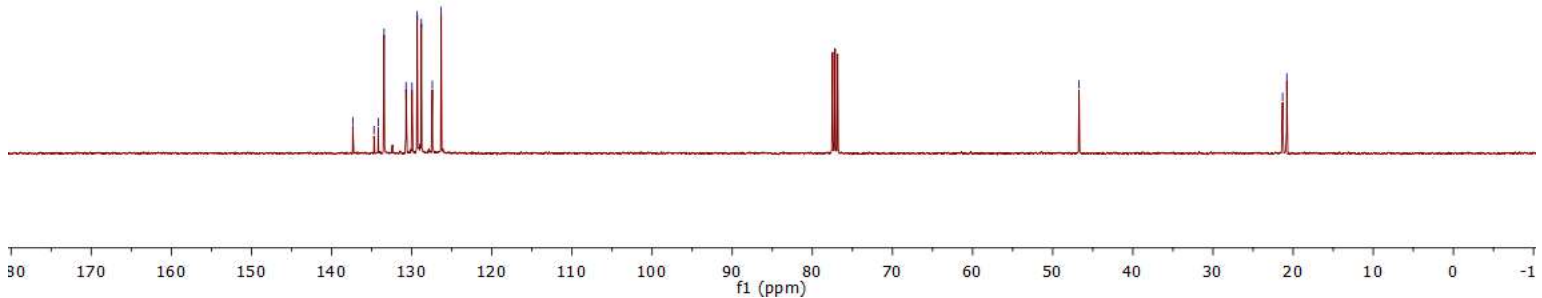
(E)-(4-(4-methoxyphenyl)but-3-en-2-yl)(phenyl)sulfane (15)

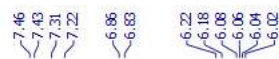

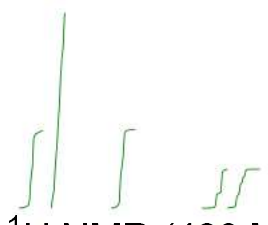

${ }^{1} \mathrm{H}$ NMR $\left(400 \mathrm{MHz}, \mathrm{CDCl}_{3}\right)$

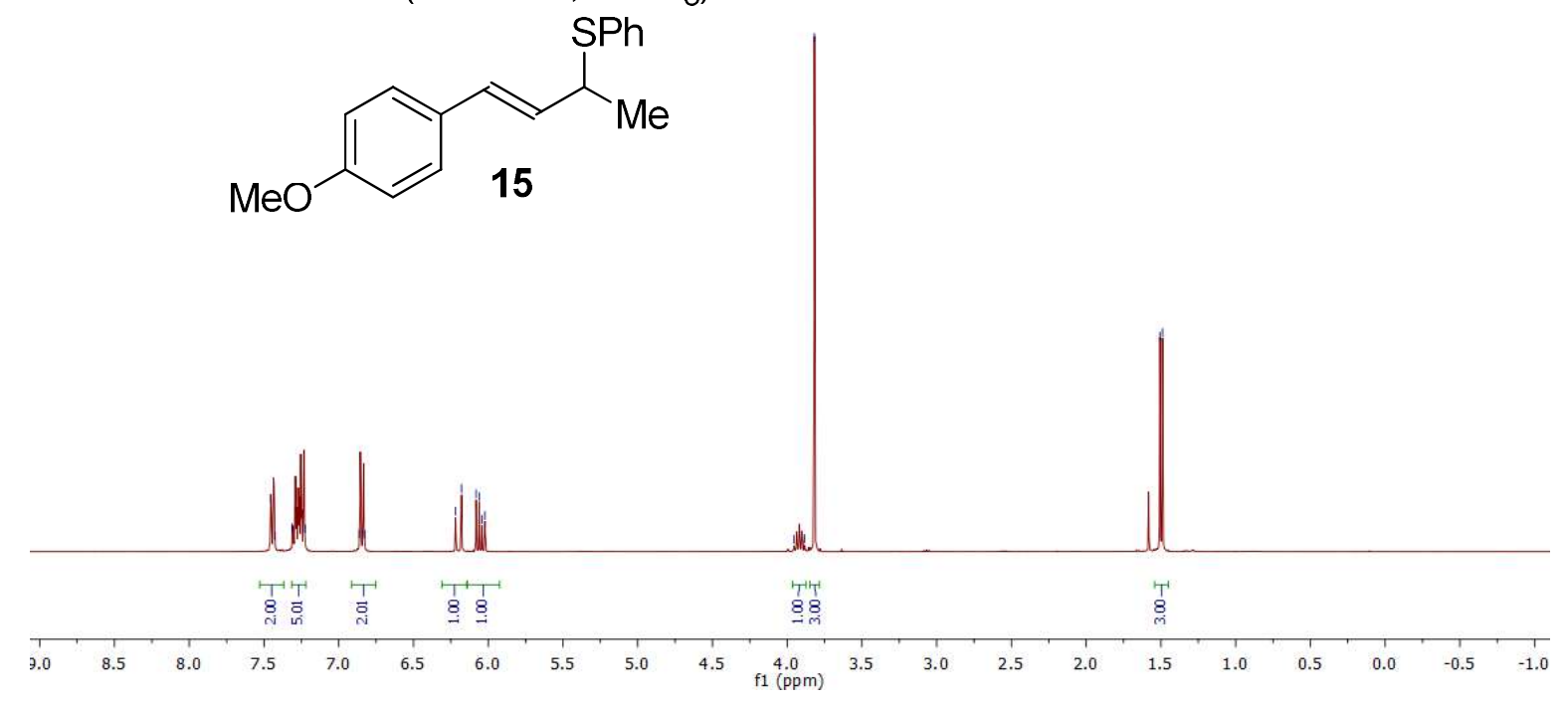

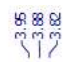
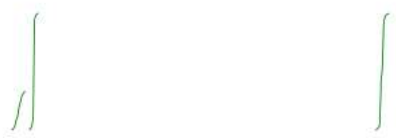

${ }^{13} \mathrm{C} \mathrm{NMR}\left(101 \mathrm{MHz}, \mathrm{CDCl}_{3}\right)$

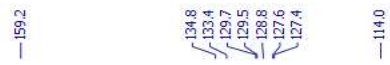

离
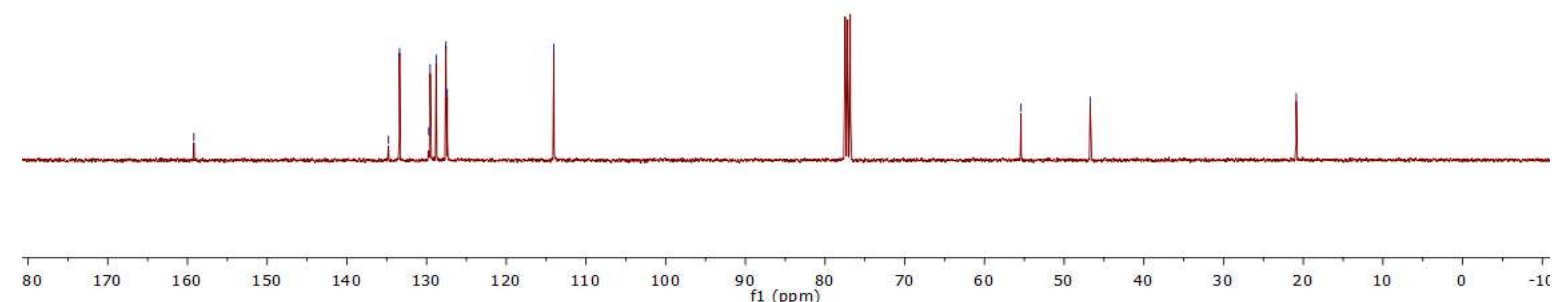
(E)-(4-(4-chlorophenyl)but-3-en-2-yl)(phenyl)sulfane (16)

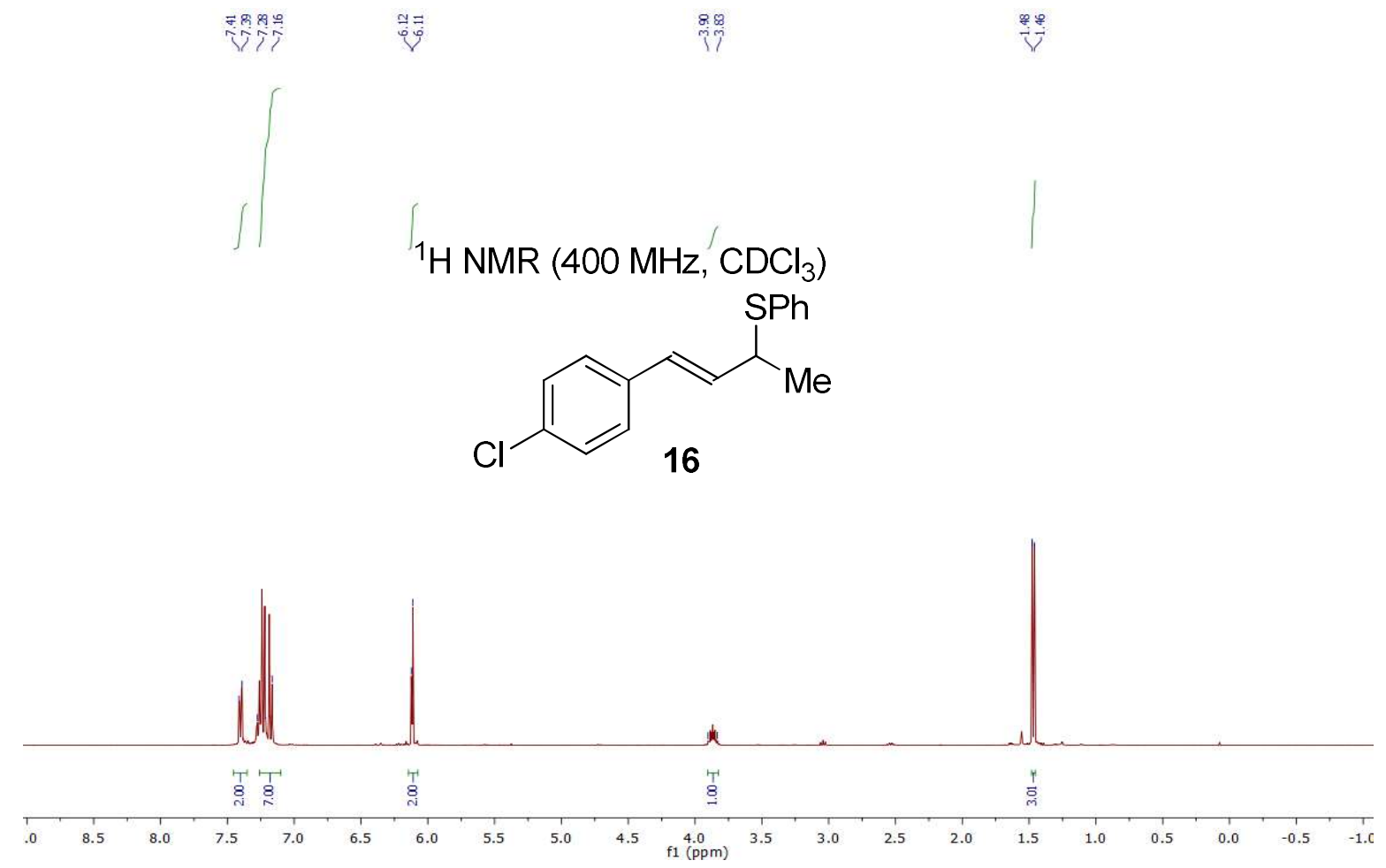

$\frac{7}{7}$

${ }^{13} \mathrm{C}$ NMR $\left(101 \mathrm{MHz}, \mathrm{CDCl}_{3}\right)$

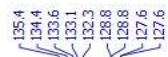

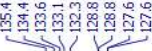

$\stackrel{\circ}{\stackrel{0}{1}} \stackrel{\circ}{1}$
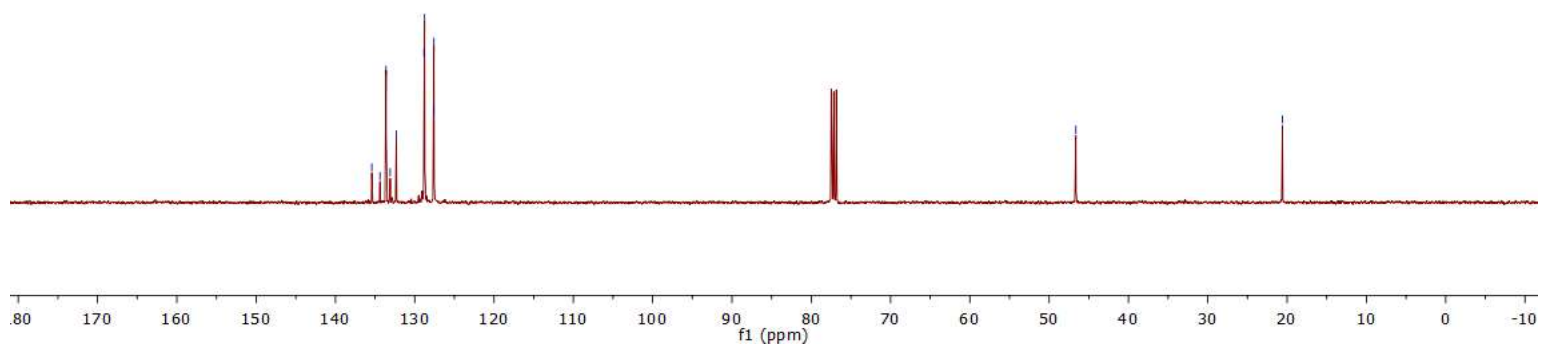
(E)-2-(3-(phenylthio)but-1-en-1-yl)furan (17)

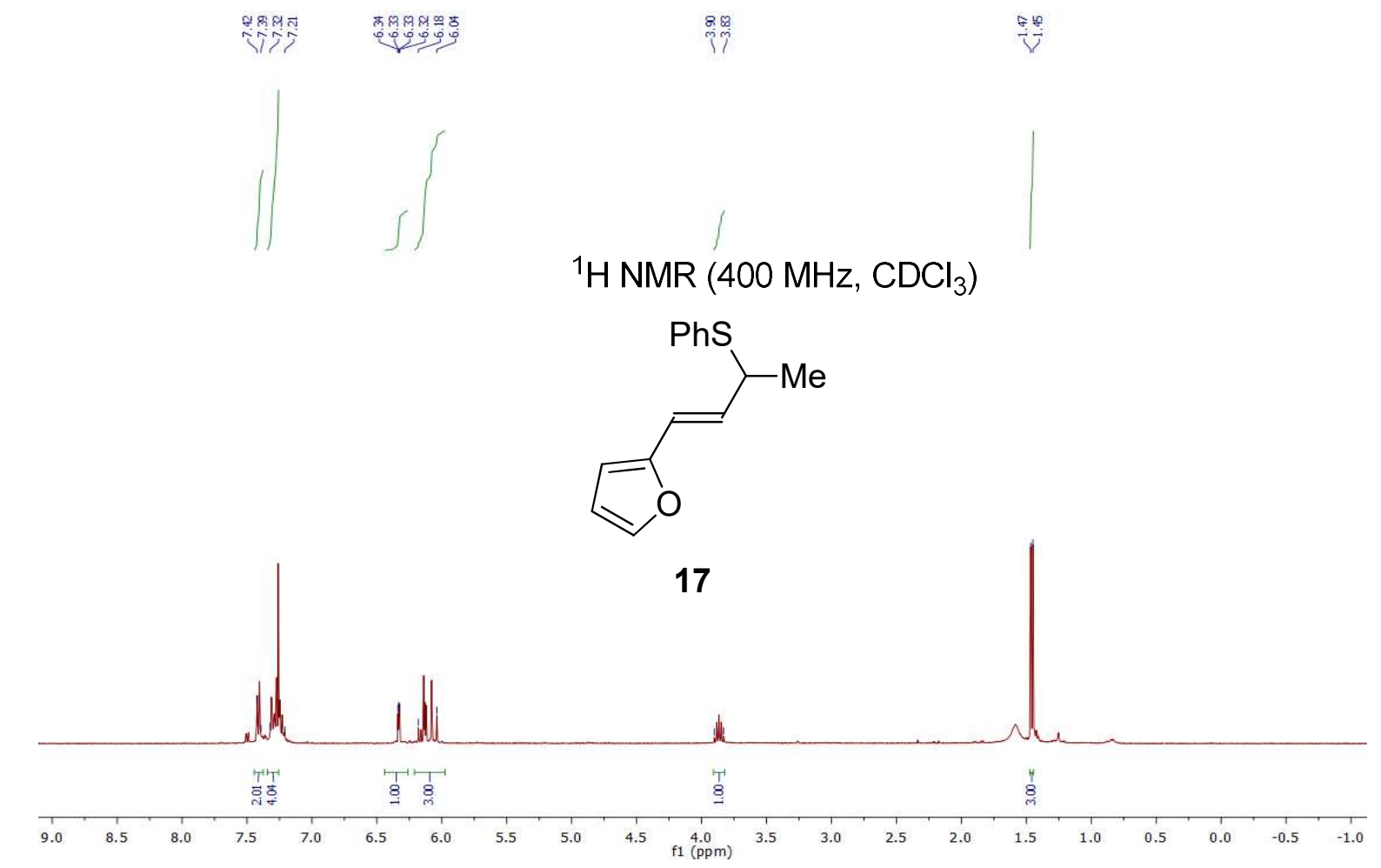

${ }^{13} \mathrm{C}$ NMR $\left(101 \mathrm{MHz}, \mathrm{CDCl}_{3}\right)$

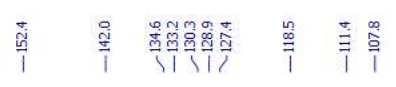

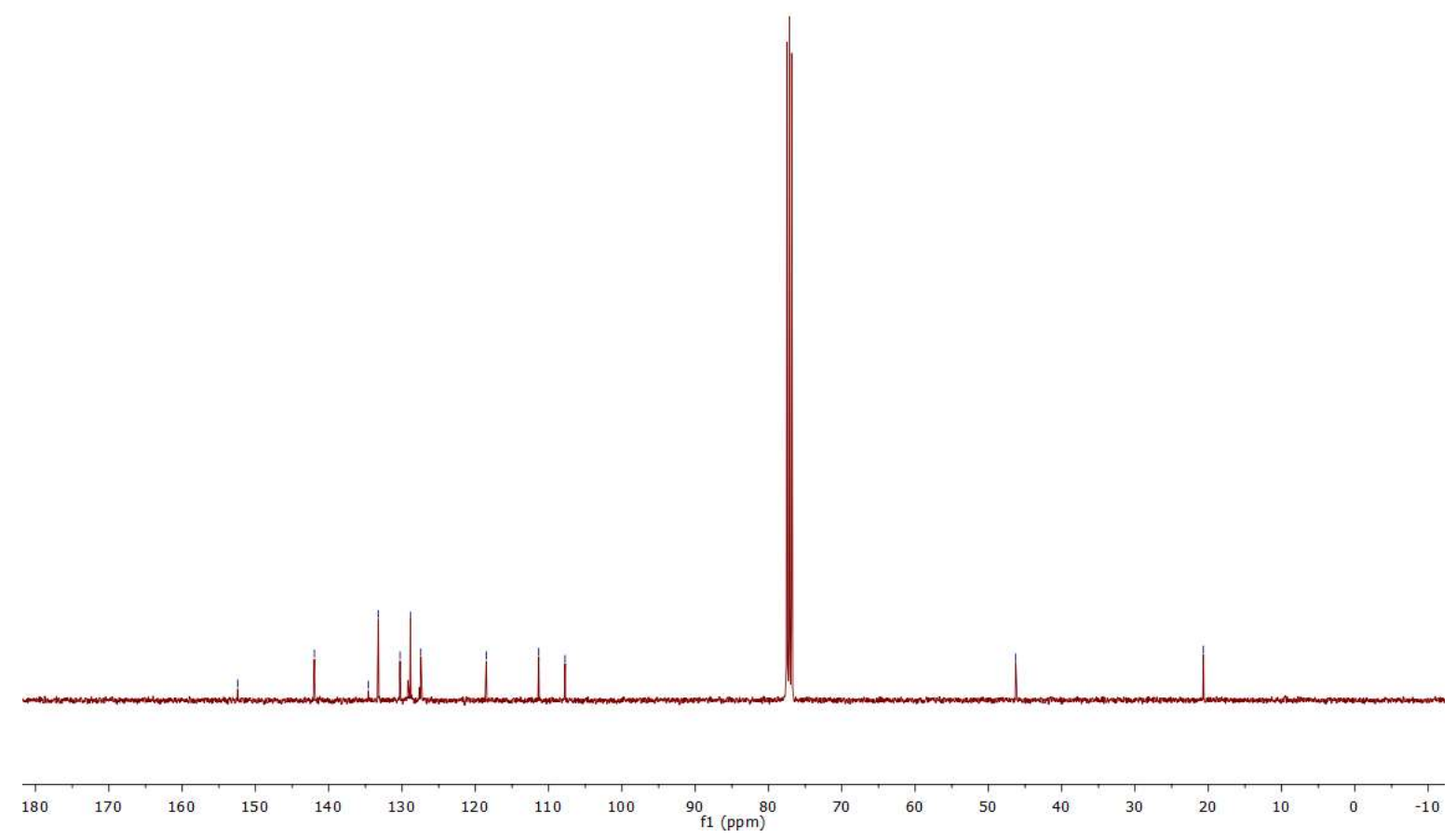


(E)-(4-(naphthalen-2-yl)but-3-en-2-yl)(phenyl)sulfane (18)

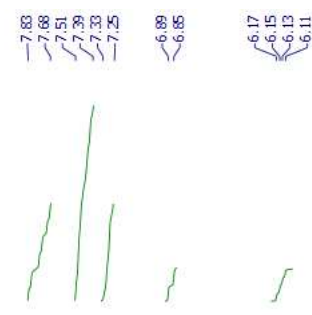

$\stackrel{88}{7}$

${ }^{1} \mathrm{H} \mathrm{NMR}\left(400 \mathrm{MHz}, \mathrm{CDCl}_{3}\right)$<smiles>CC(C=Cc1ccc2ccccc2c1)c1ccccc1</smiles>

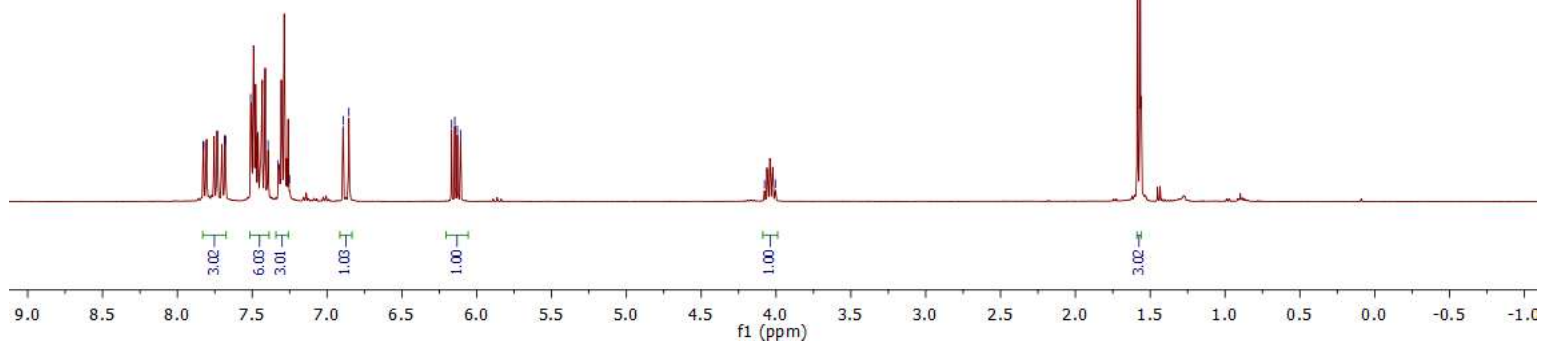

${ }^{13} \mathrm{C}$ NMR $\left(101 \mathrm{MHz}, \mathrm{CDCl}_{3}\right)$

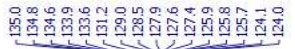
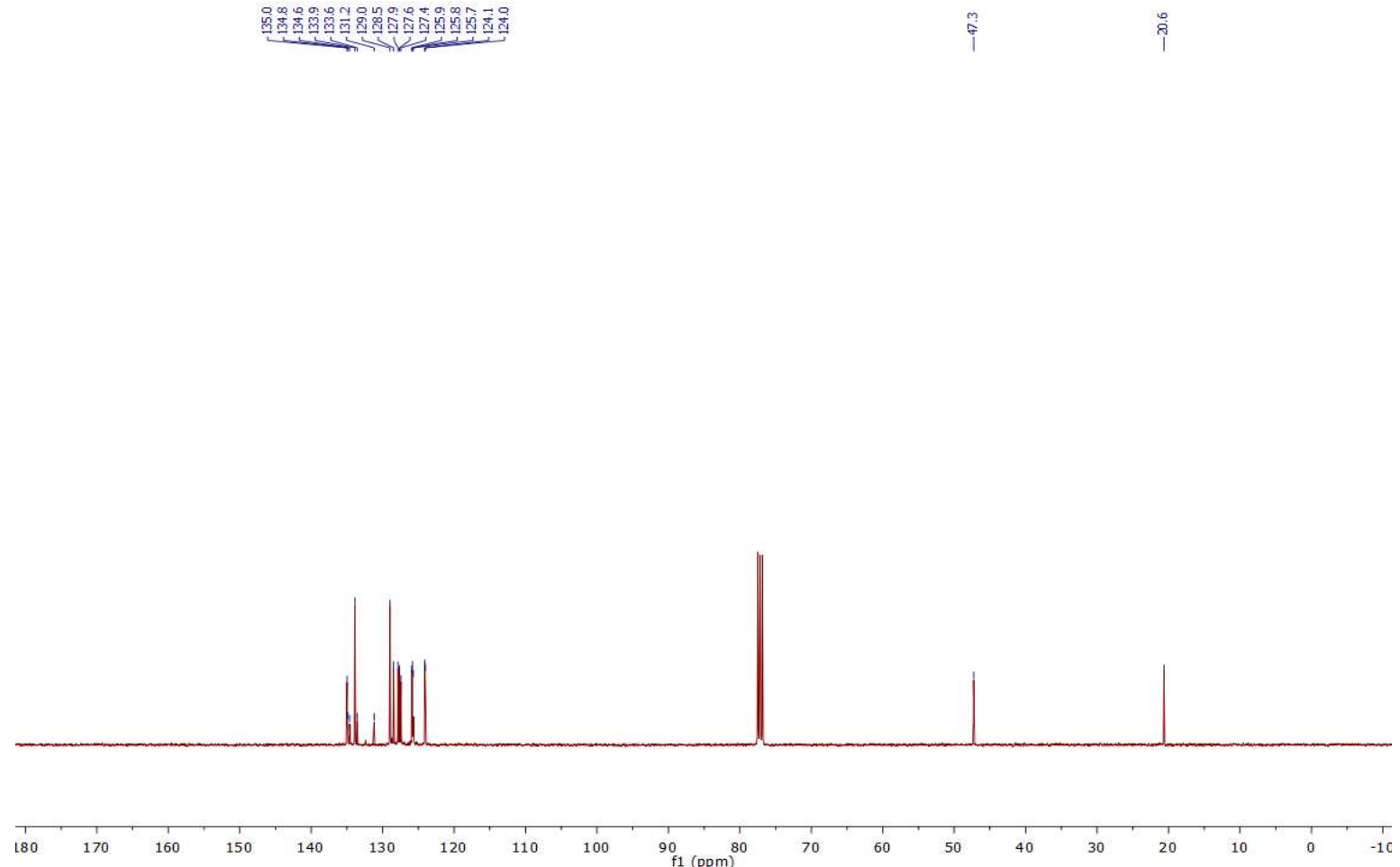
(E)-(4-(ferrocene-2-yl)but-3-en-2-yl)(phenyl)sulfane (19)

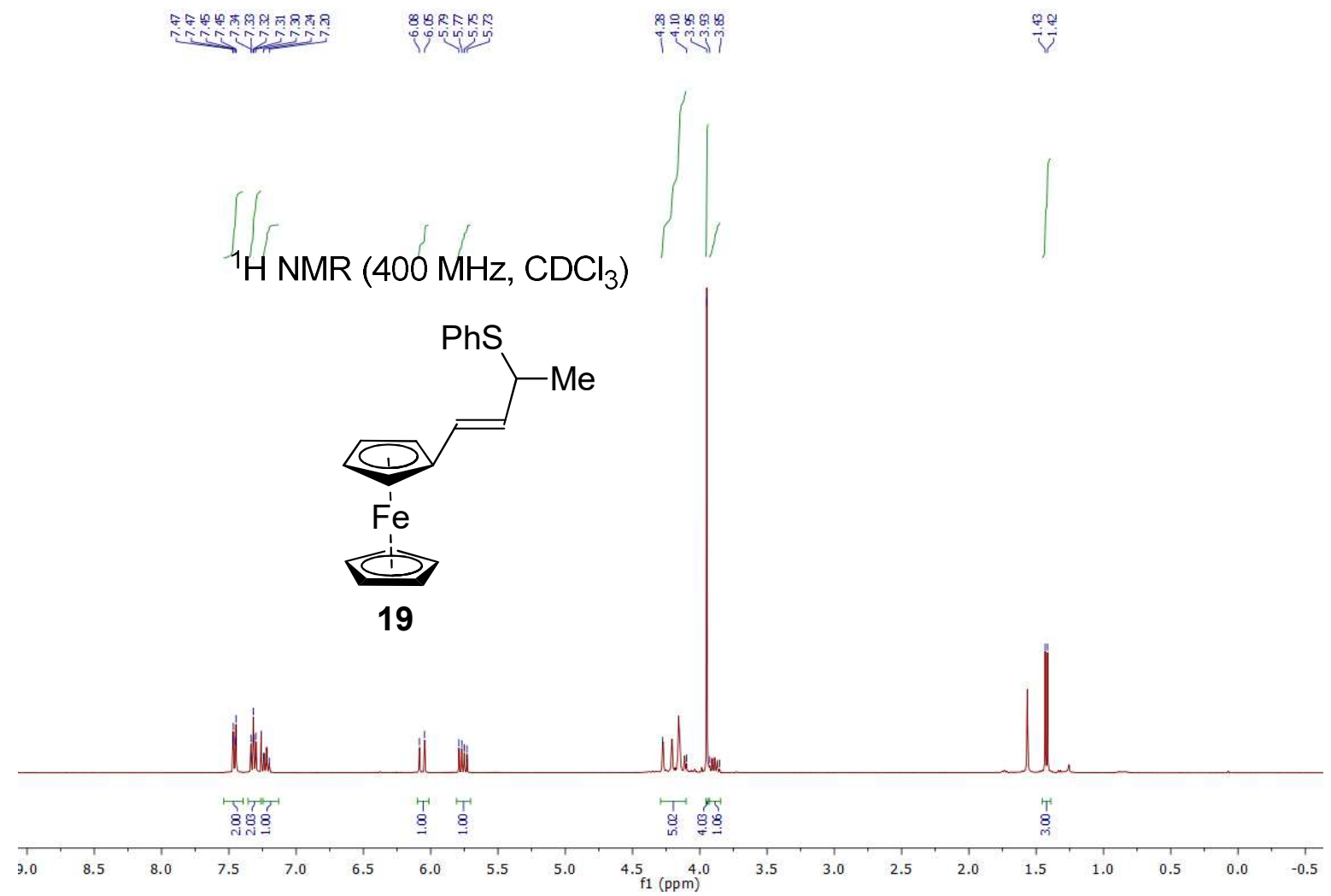

${ }^{13} \mathrm{C}$ NMR $\left(101 \mathrm{MHz}, \mathrm{CDCl}_{3}\right)$

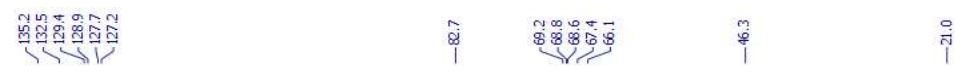

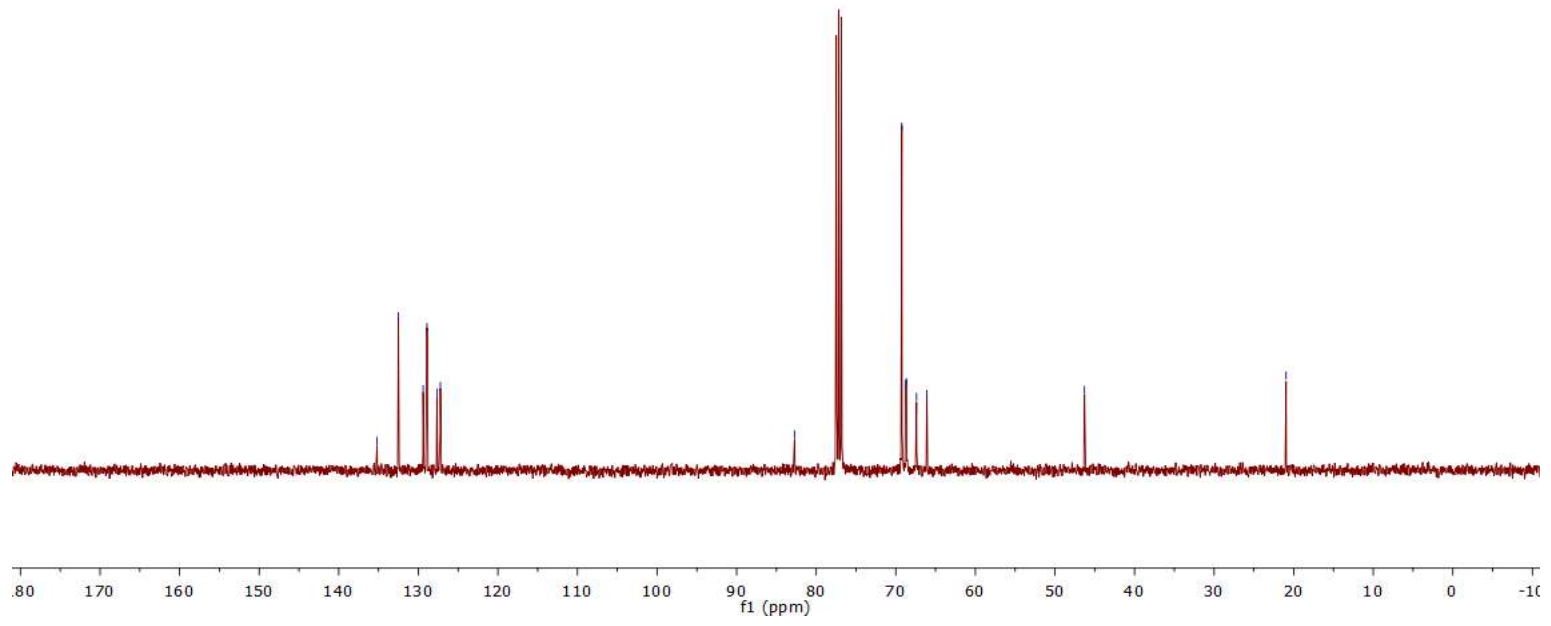


(1-(ferrocene-2-yl)butane-1,3-diyl)bis(phenylsulfane) (19')

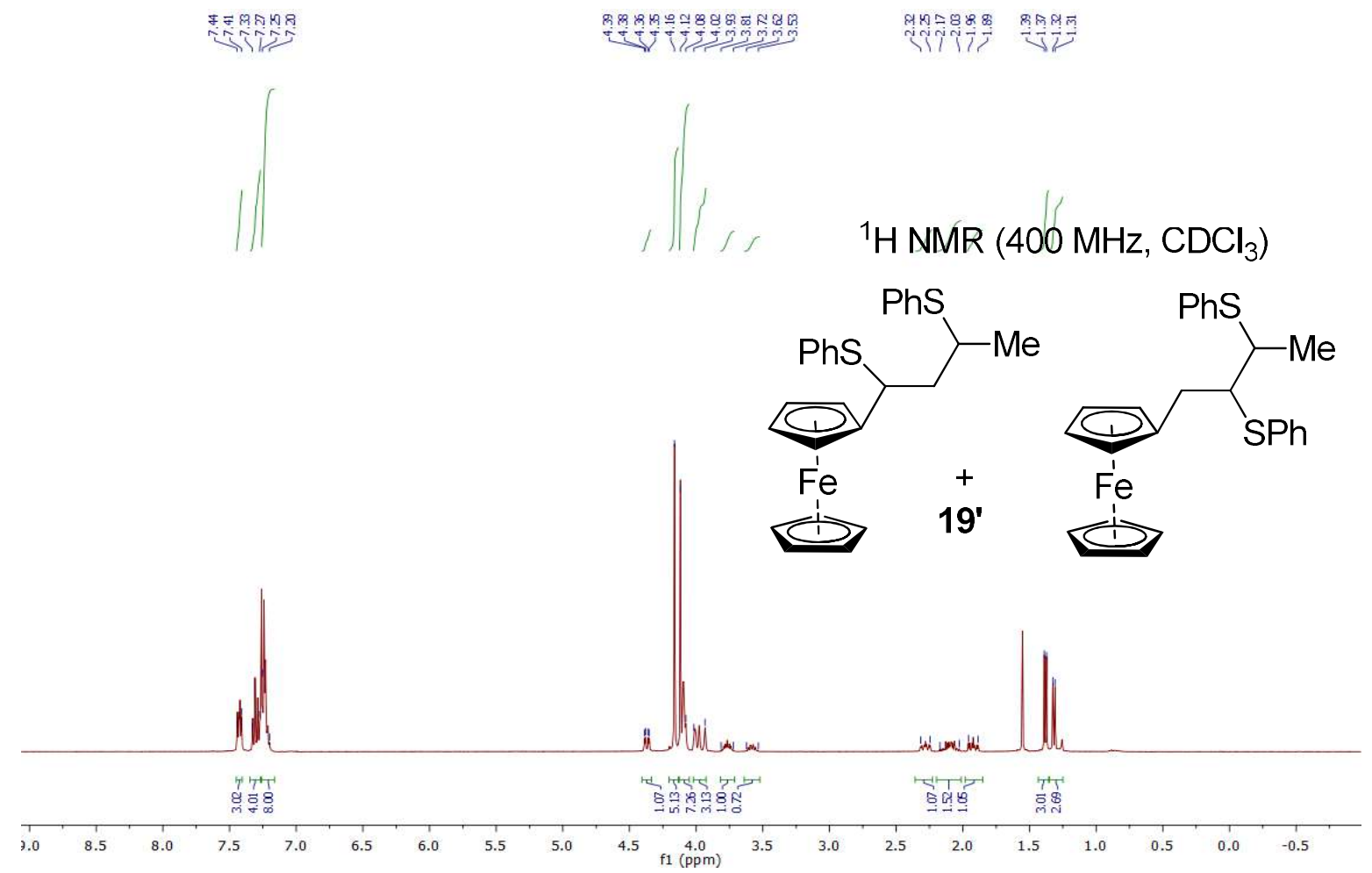

${ }^{13} \mathrm{C}$ NMR $\left(101 \mathrm{MHz}, \mathrm{CDCl}_{3}\right)$

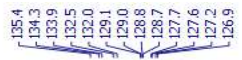

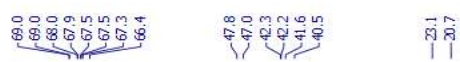

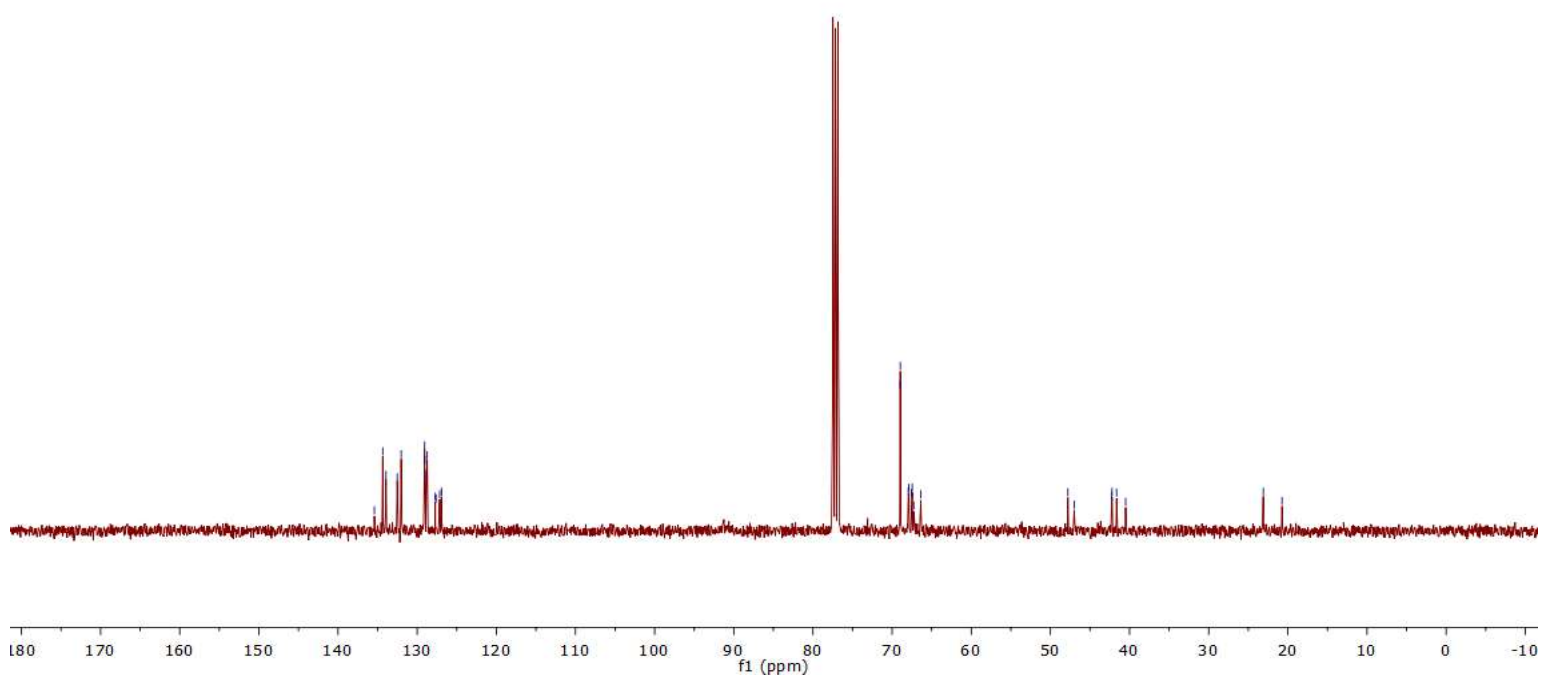


oct-3-en-2-yl(phenyl)sulfane, oct-2-en-1-yl(phenyl)sulfane \& oct-3-en-1yl(phenyl)sulfane $(20+20 '+20 ")$

${ }^{1} \mathrm{H}$ NMR (400 MHz, $\left.\mathrm{CDCl}_{3}\right)$

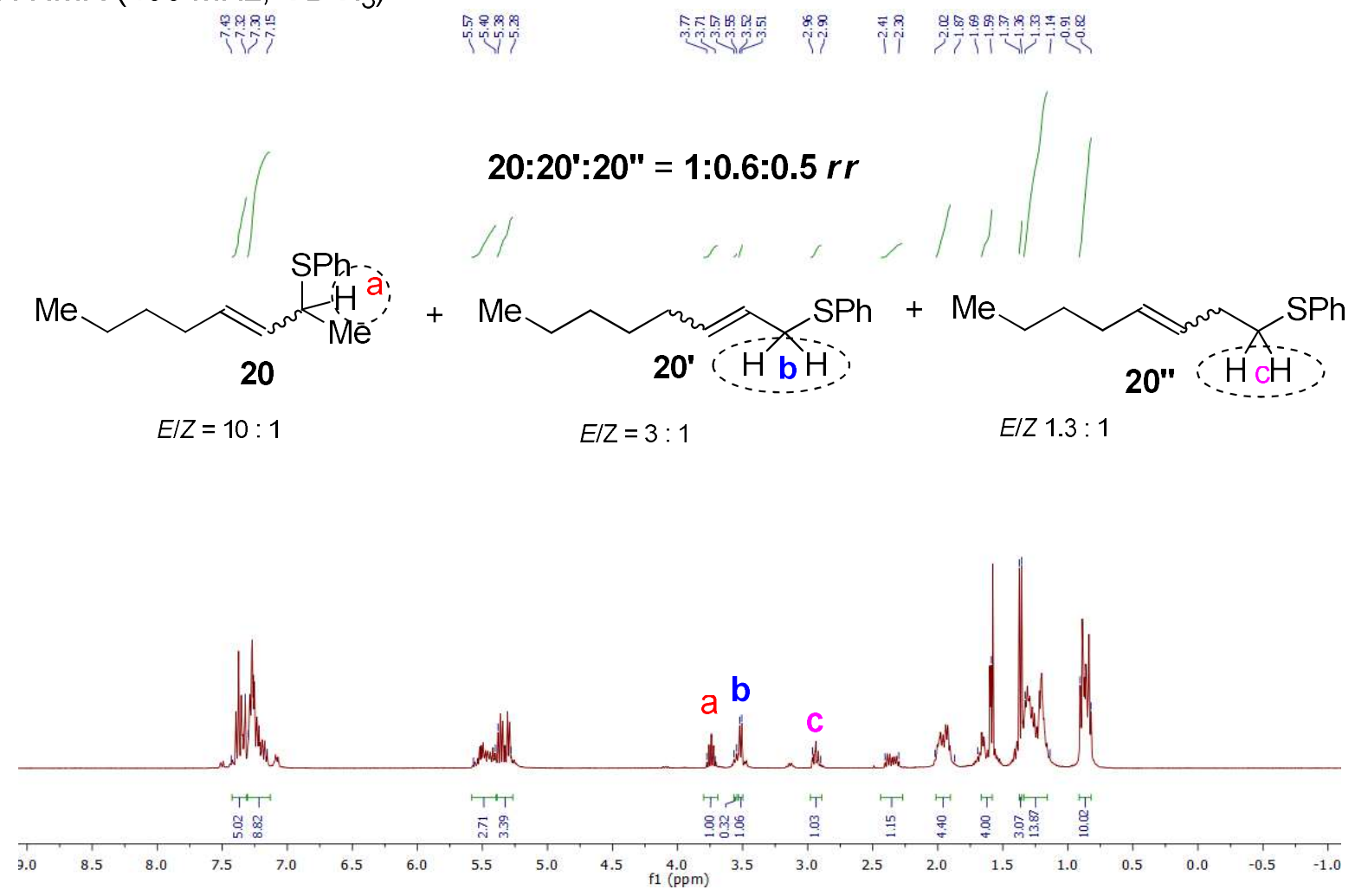

${ }^{13} \mathrm{C}$ NMR $\left(101 \mathrm{MHz}, \mathrm{CDCl}_{3}\right)$

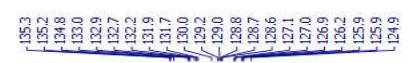

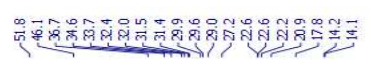

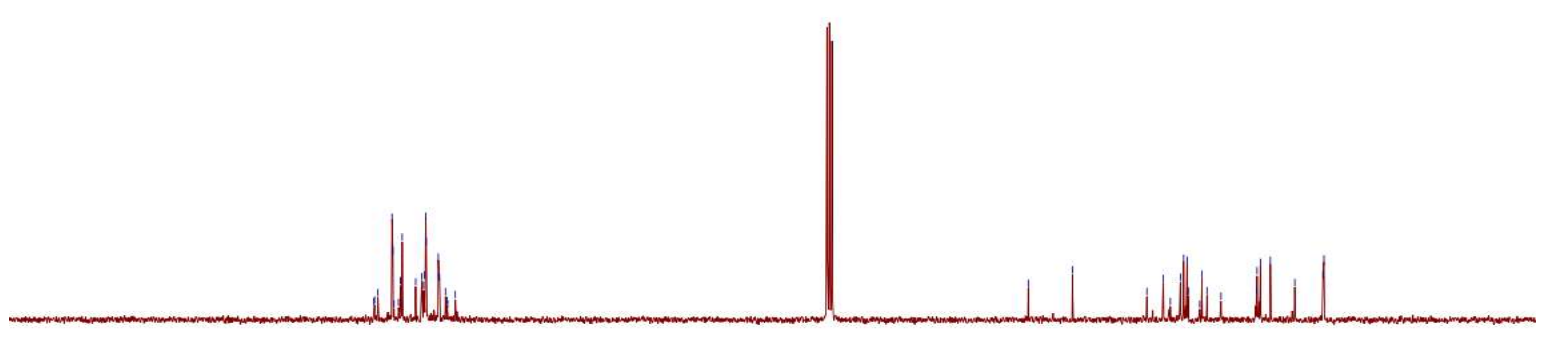

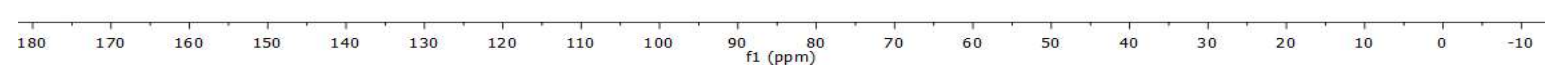




\section{(4-cyclohexylbut-3-en-2-yl)(phenyl)sulfane (4-cyclohexylbut-2-en-1-yl)(phenyl)sulfane}

\& (4-cyclohexylbut-3-en-1-yl)(phenyl)sulfane $\left(21+21^{\prime}+21 "\right)$

${ }^{1} \mathrm{H}$ NMR $\left(400 \mathrm{MHz}, \mathrm{CDCl}_{3}\right)$

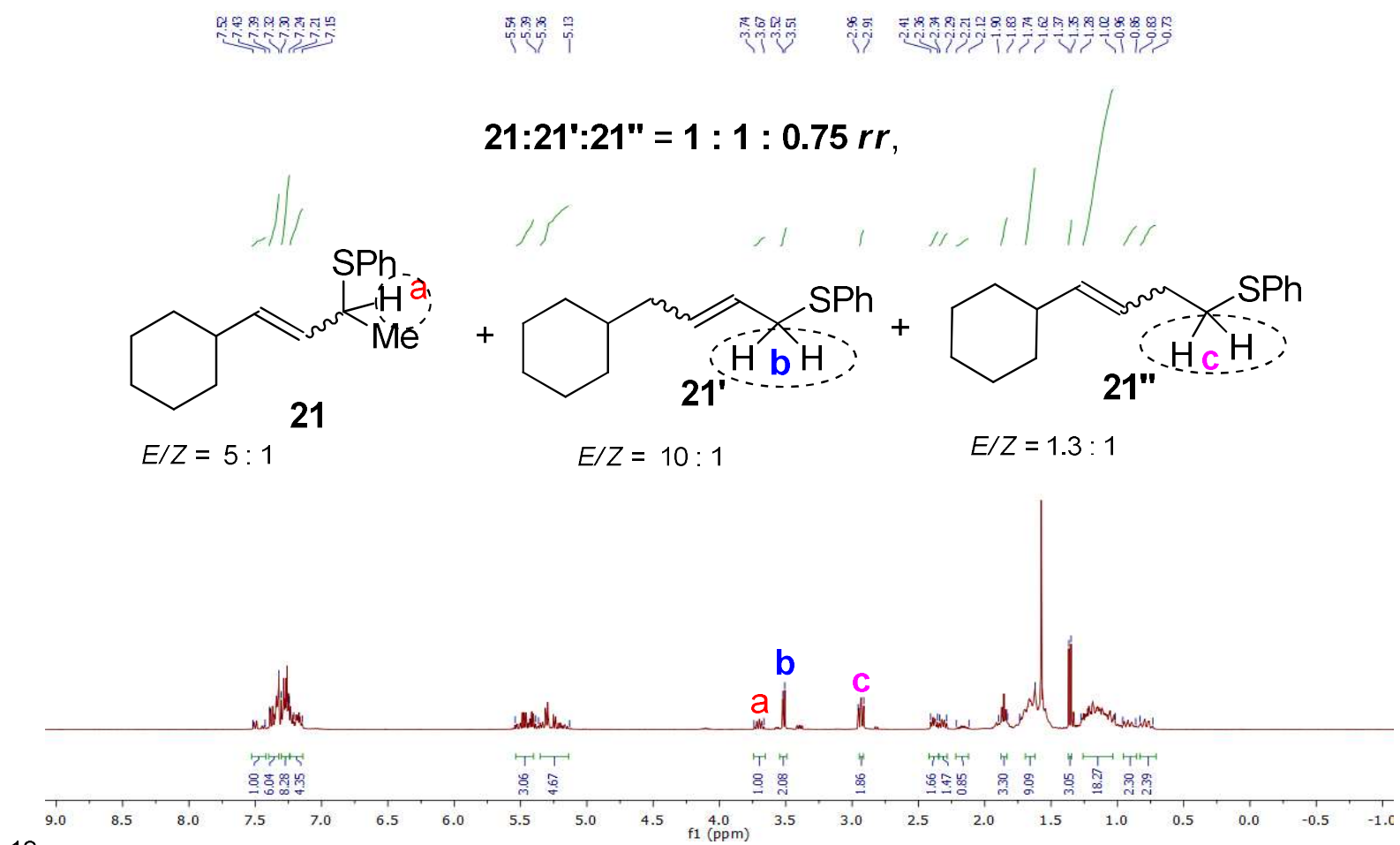

${ }^{13} \mathrm{C}$ NMR $\left(101 \mathrm{MHz} \mathrm{CDCl}_{3}\right)$

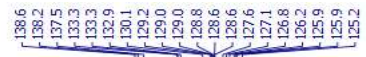

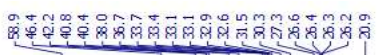

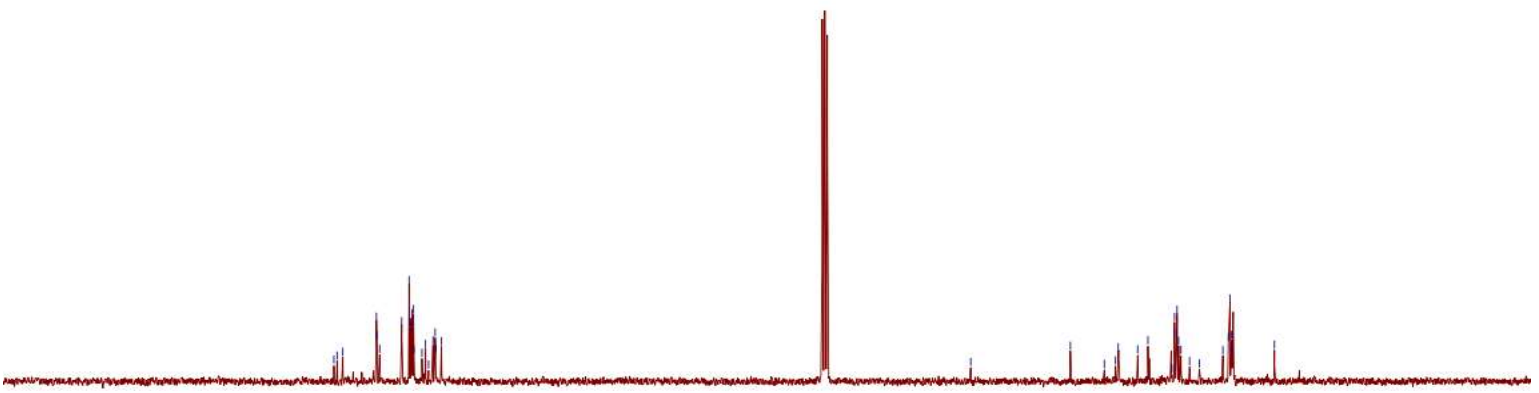

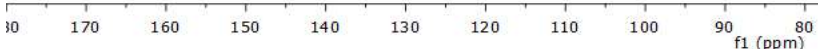


phenyl(6-phenylhex-2-en-1-yl)sulfane and phenyl(6-phenylhex-3-en-1-yl)sulfane (22 + 22')

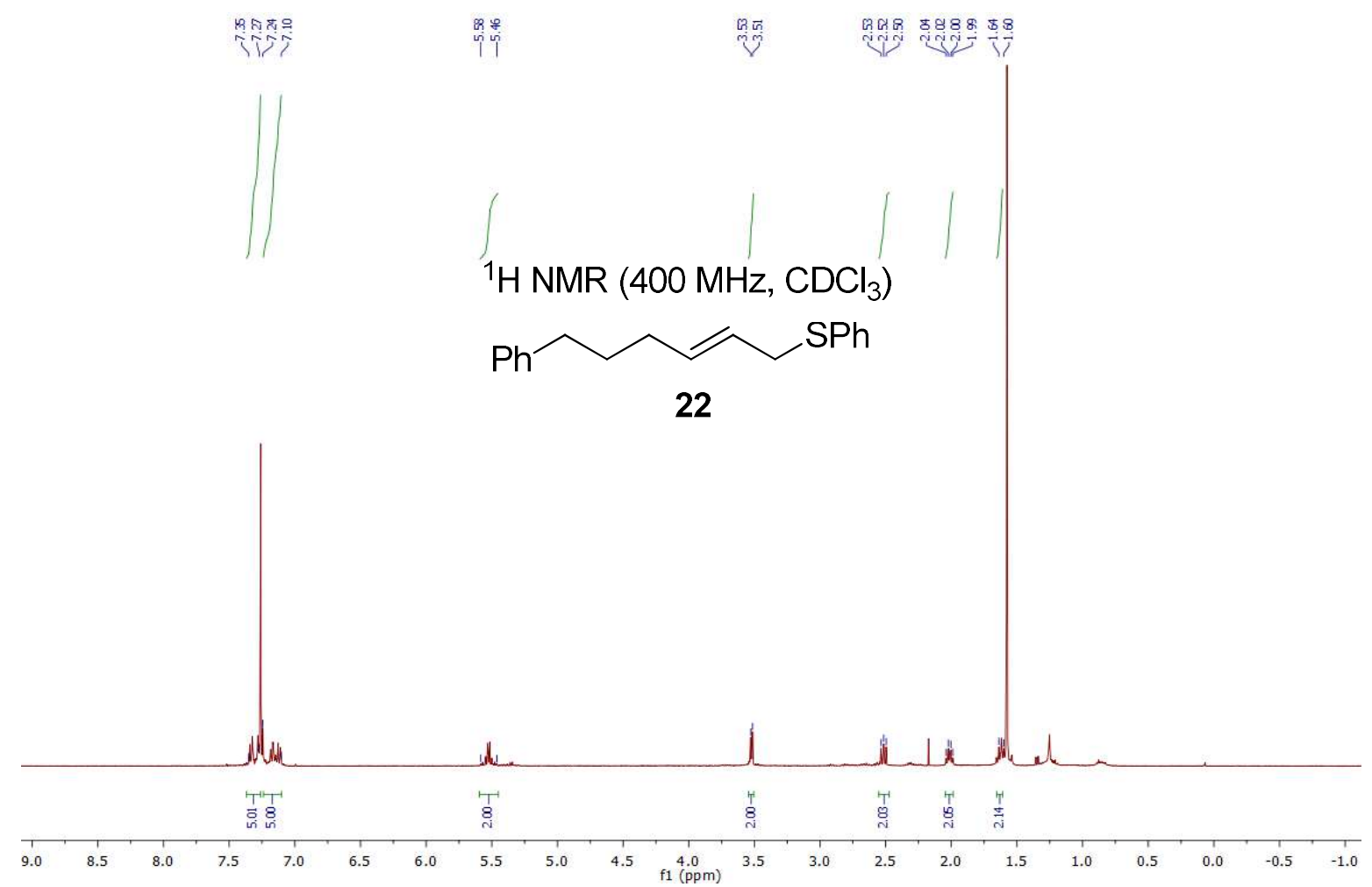

${ }^{13} \mathrm{C} \mathrm{NMR}\left(101 \mathrm{MHz}, \mathrm{CDCl}_{3}\right)$

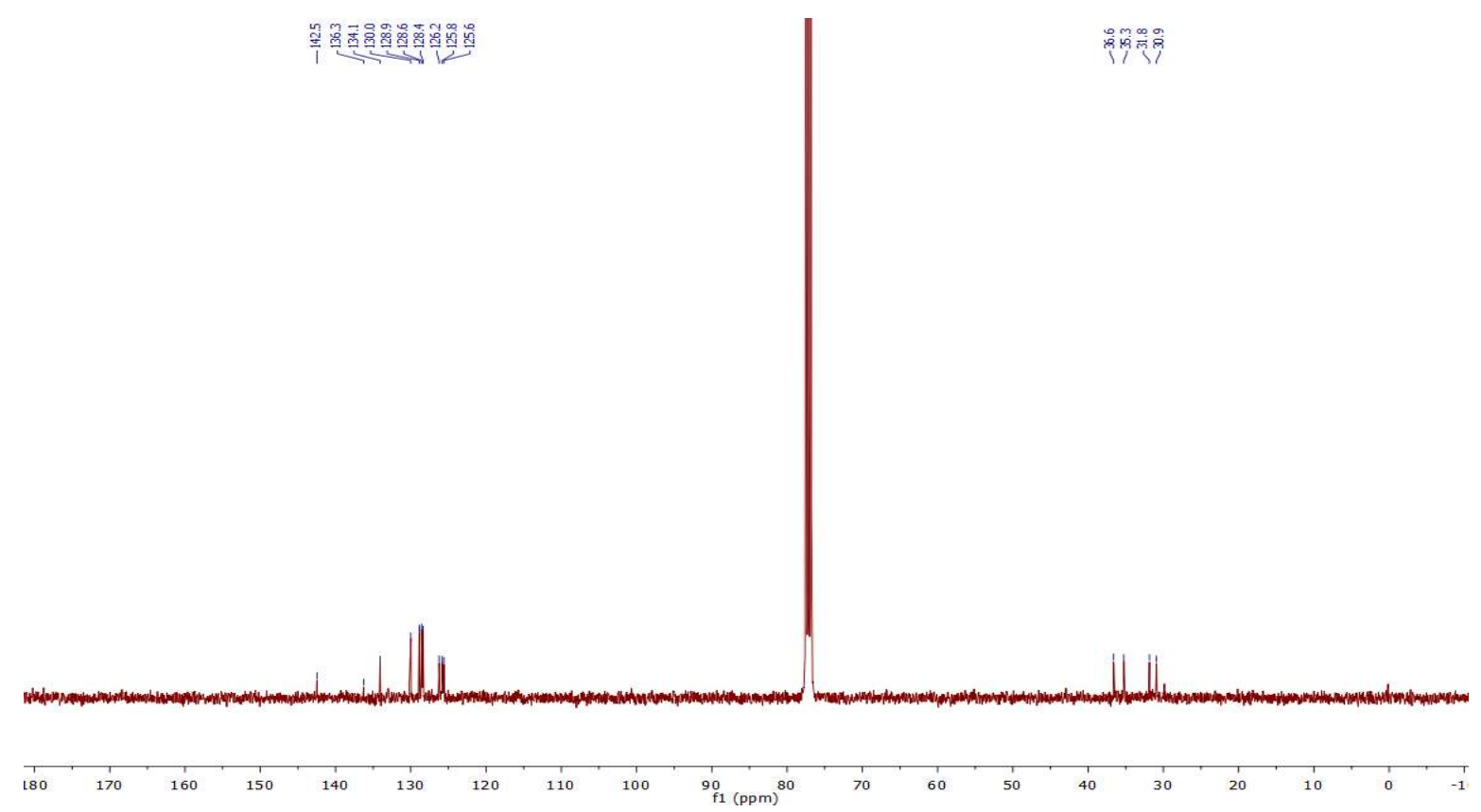


${ }^{1} \mathrm{H}$ NMR $\left(400 \mathrm{MHz}, \mathrm{CDCl}_{3}\right)$

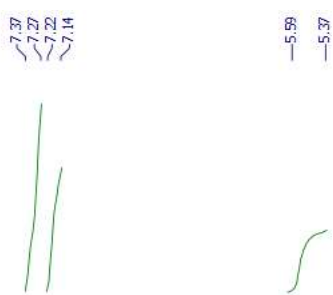

$22: 22^{\prime}=1: 1$

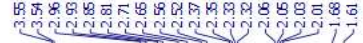

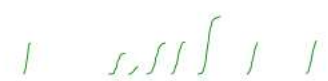

22', $E / Z=3: 1$

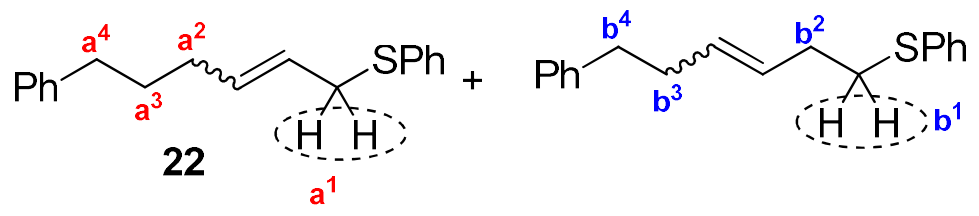

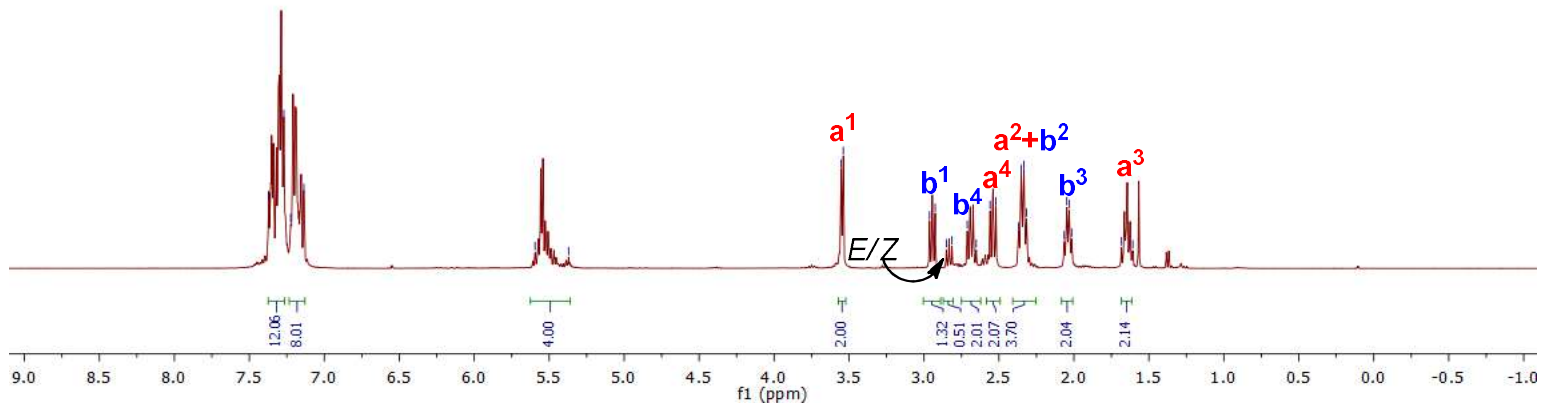

${ }^{13} \mathrm{C} \mathrm{NMR}\left(101 \mathrm{MHz}, \mathrm{CDCl}_{3}\right)$

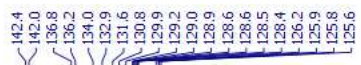
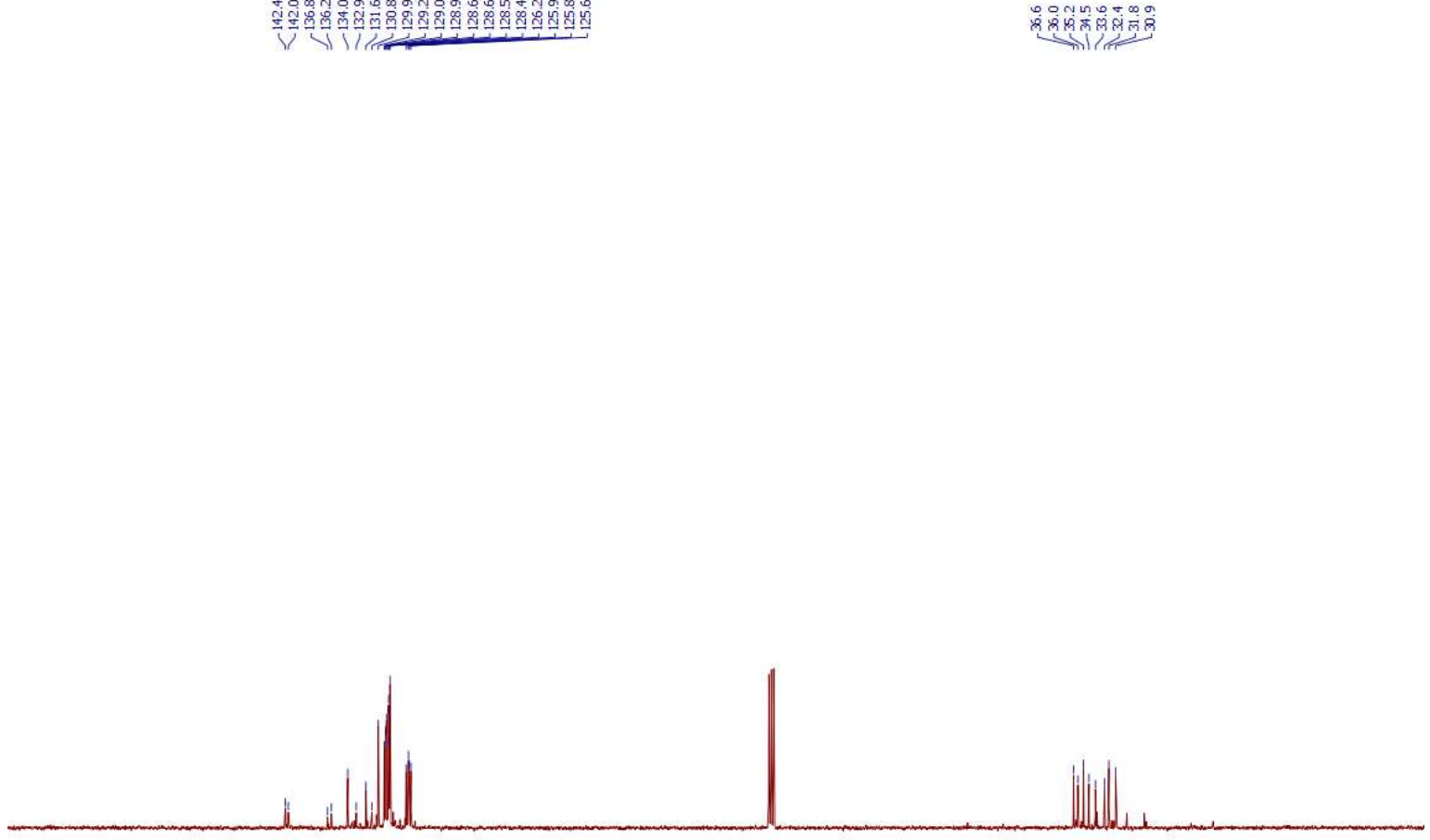

\begin{tabular}{|c|c|c|c|c|c|c|c|c|c|c|c|c|c|c|c|c|}
\hline 170 & 160 & 150 & 140 & 130 & 120 & 110 & 100 & ${ }^{90}{ }_{\mathrm{f} 1(\mathrm{ppm})}{ }^{80}$ & 70 & 60 & 50 & 40 & 30 & 20 & 10 & 0 \\
\hline
\end{tabular}


(E)-phenyl(4-phenylpent-3-en-2-yl)sulfane (23)
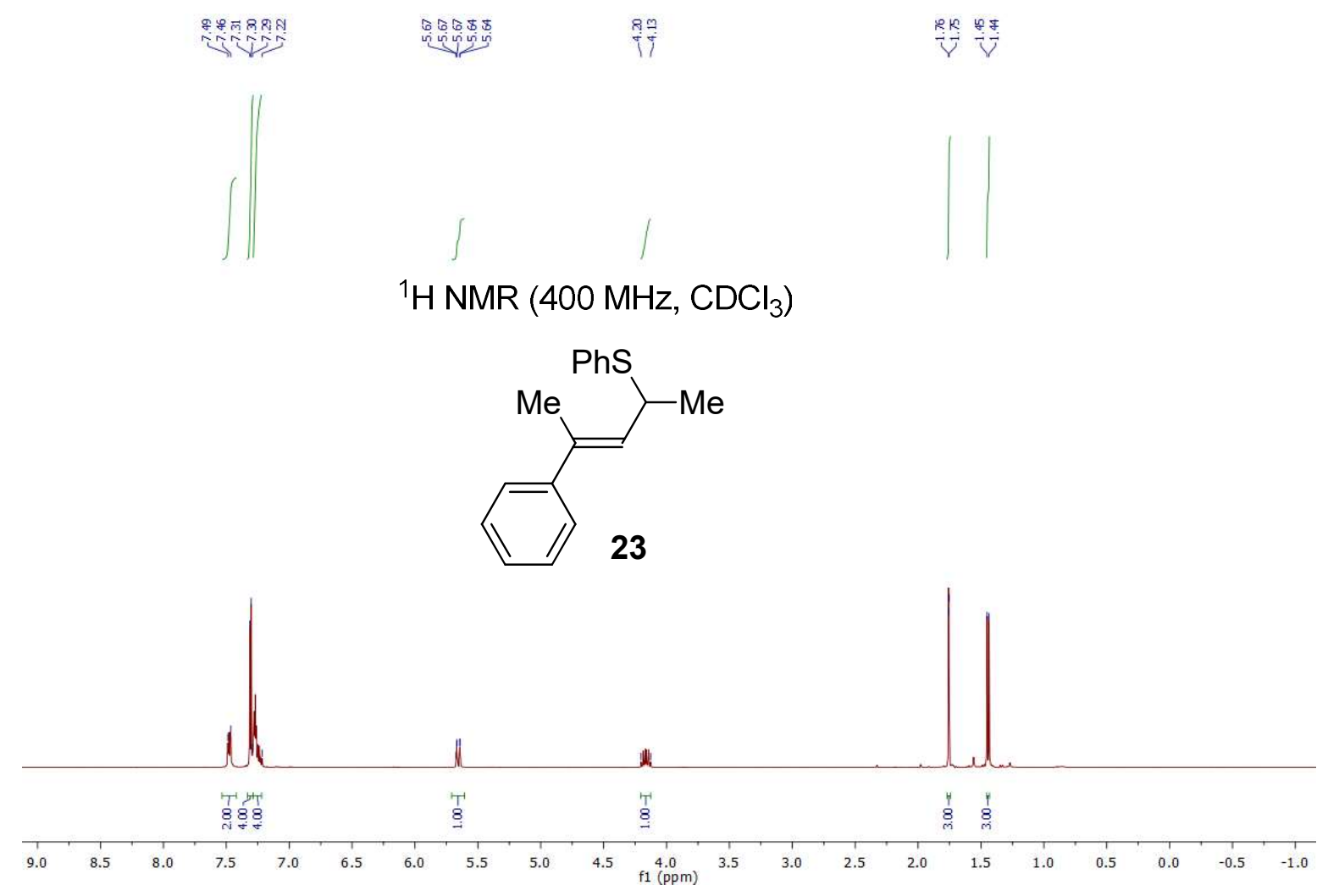

${ }^{13} \mathrm{C}$ NMR (101 MHz, $\left.\mathrm{CDCl}_{3}\right)$

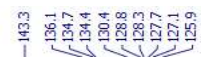

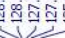

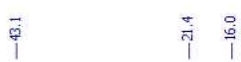
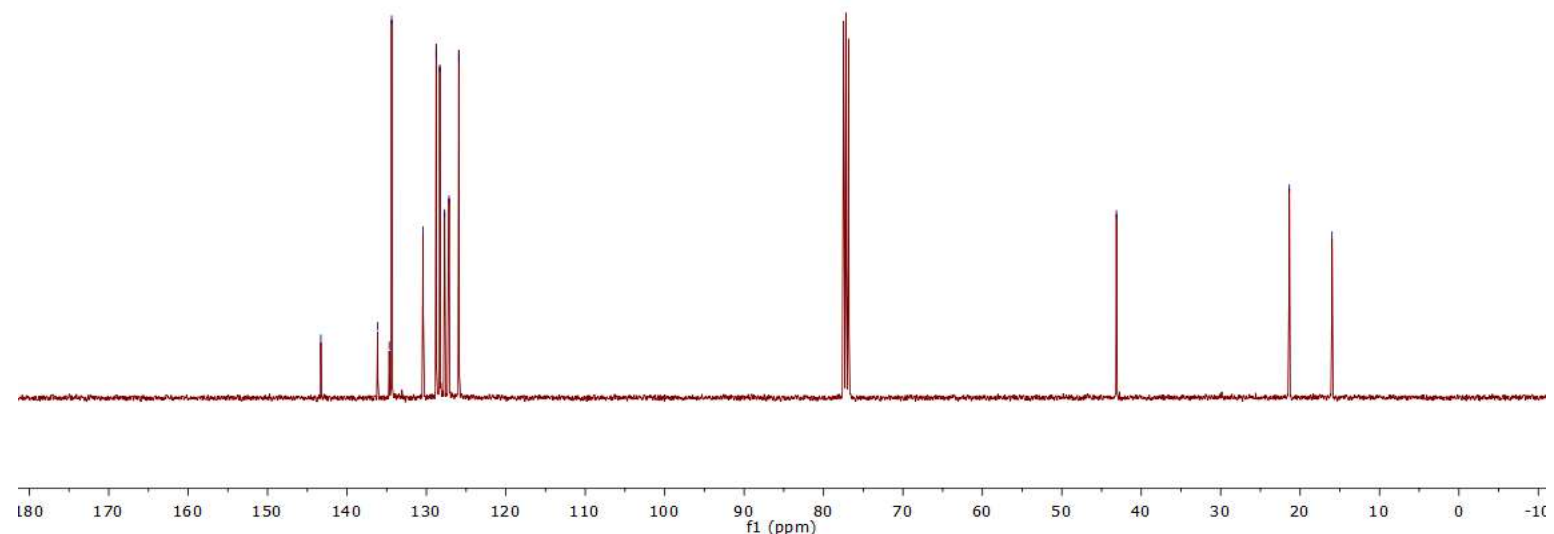


\section{(4,4-diphenylbut-3-en-2-yl)(phenyl)sulfane (24)}

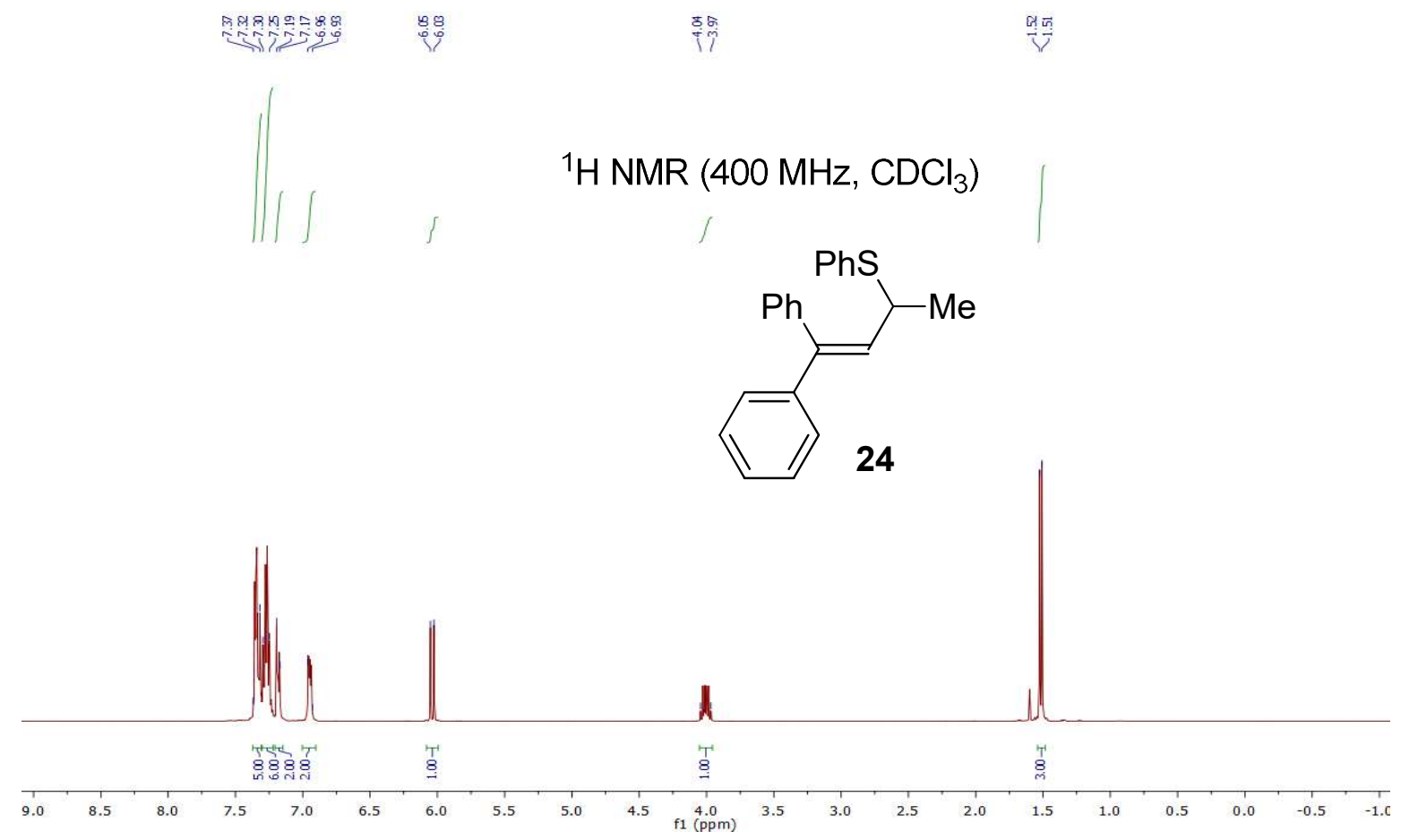

${ }^{13} \mathrm{C}$ NMR (101 MHz, $\mathrm{CDCl}_{3}$ )
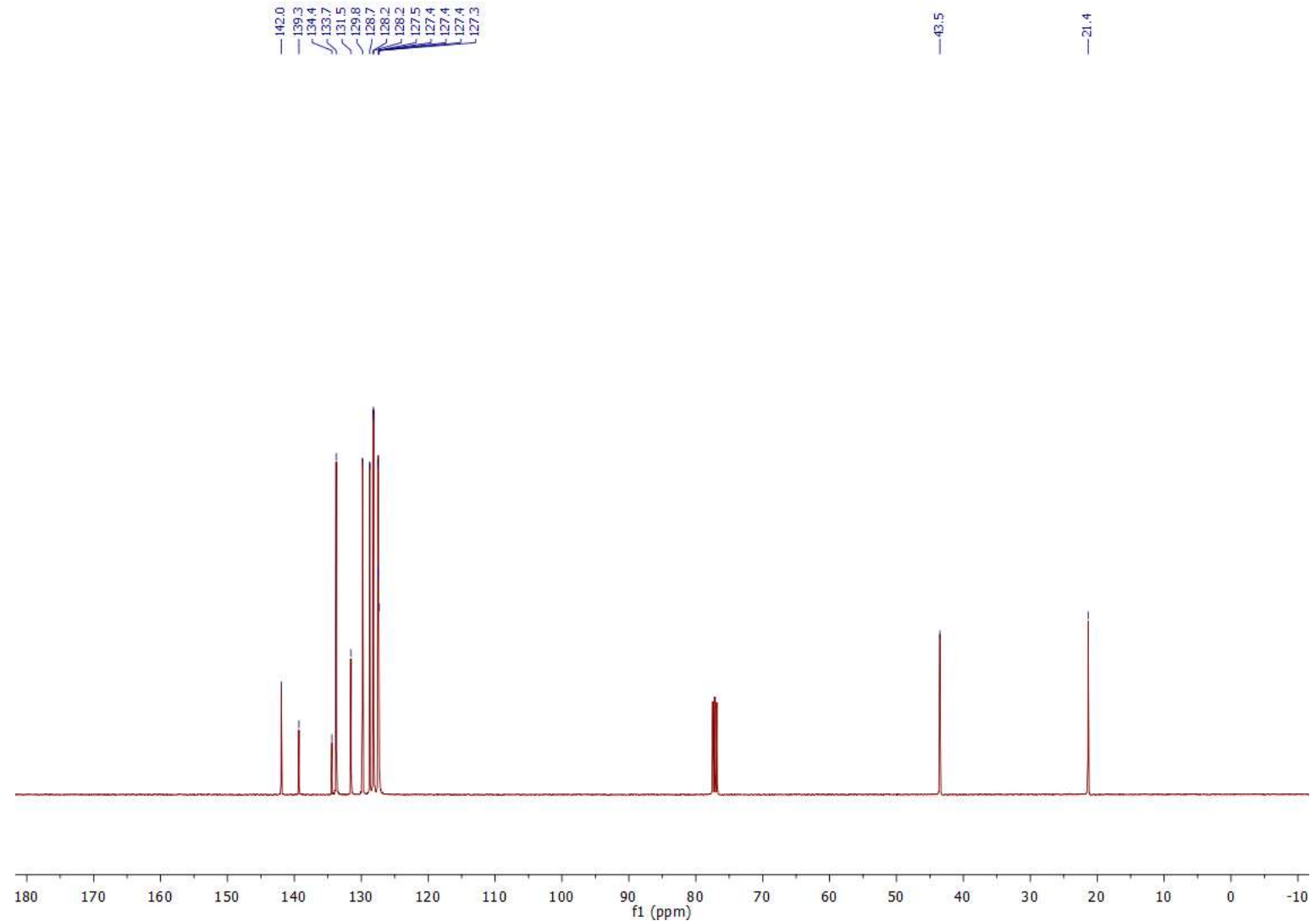
(1-cyclohexylidenepropan-2-yl)(phenyl)sulfane (25)
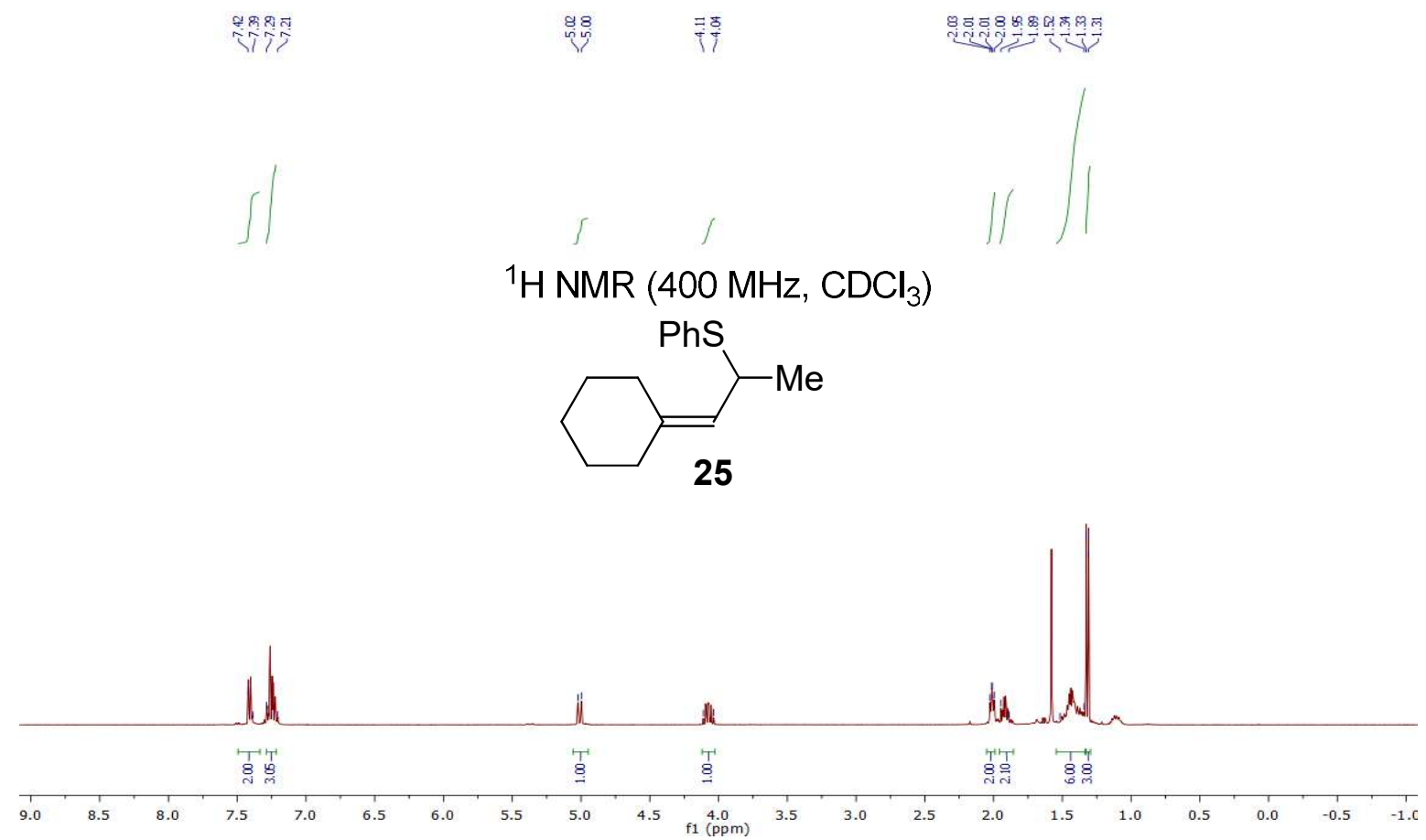

${ }^{13} \mathrm{C}$ NMR (101 MHz, $\left.\mathrm{CDCl}_{3}\right)$

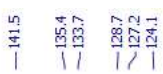

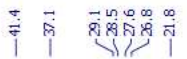
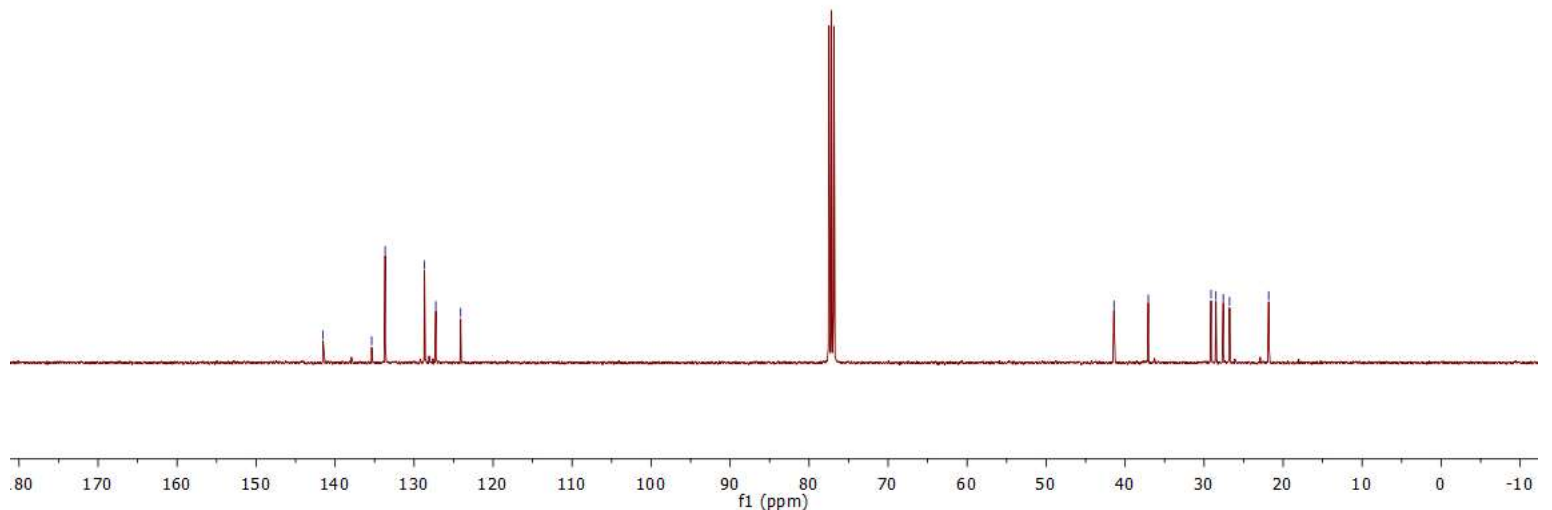
(E)-phenyl(1-phenylpent-1-en-3-yl)sulfane (26)

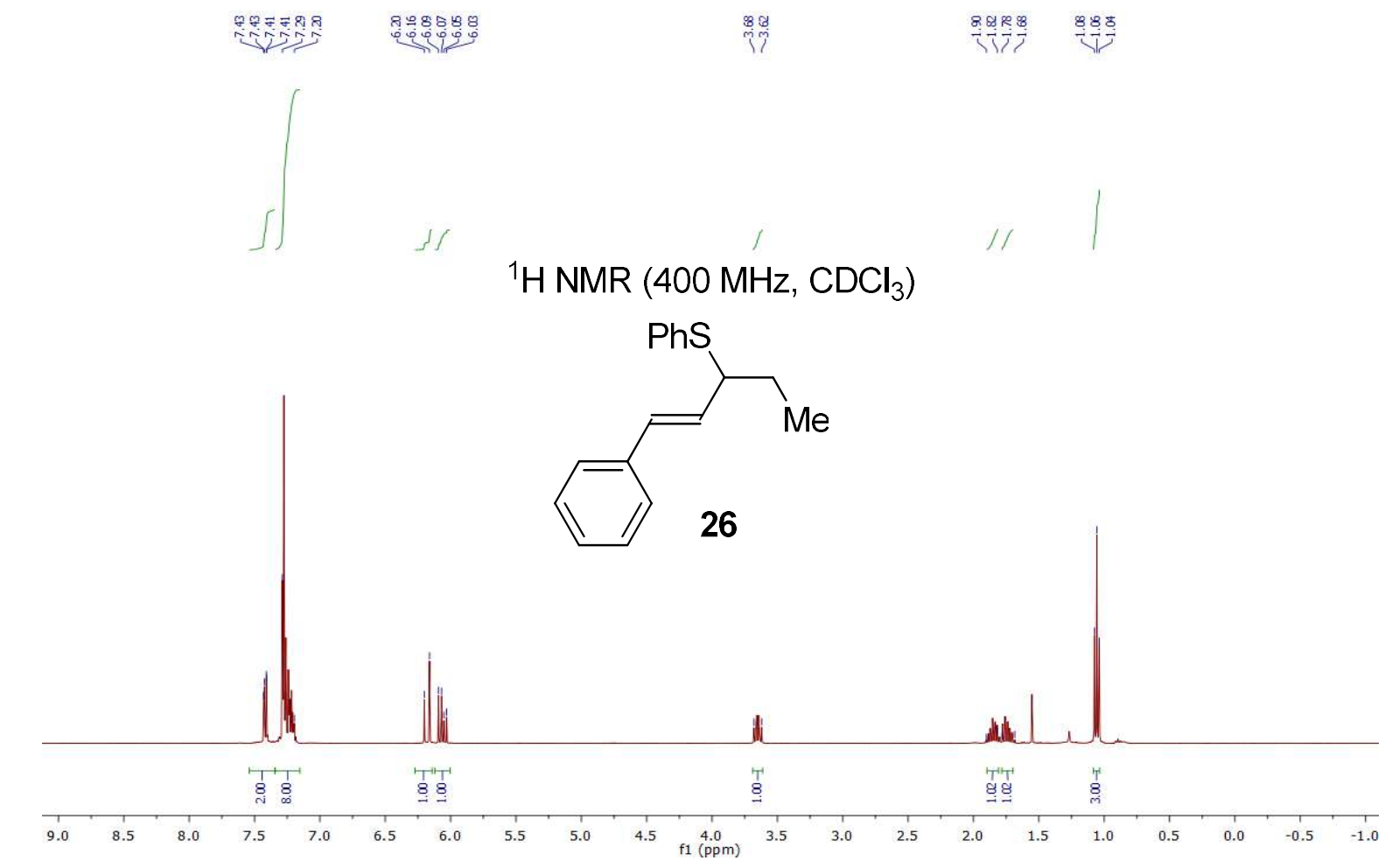

${ }^{13} \mathrm{C} \mathrm{NMR}\left(101 \mathrm{MHz}, \mathrm{CDCl}_{3}\right)$

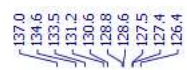
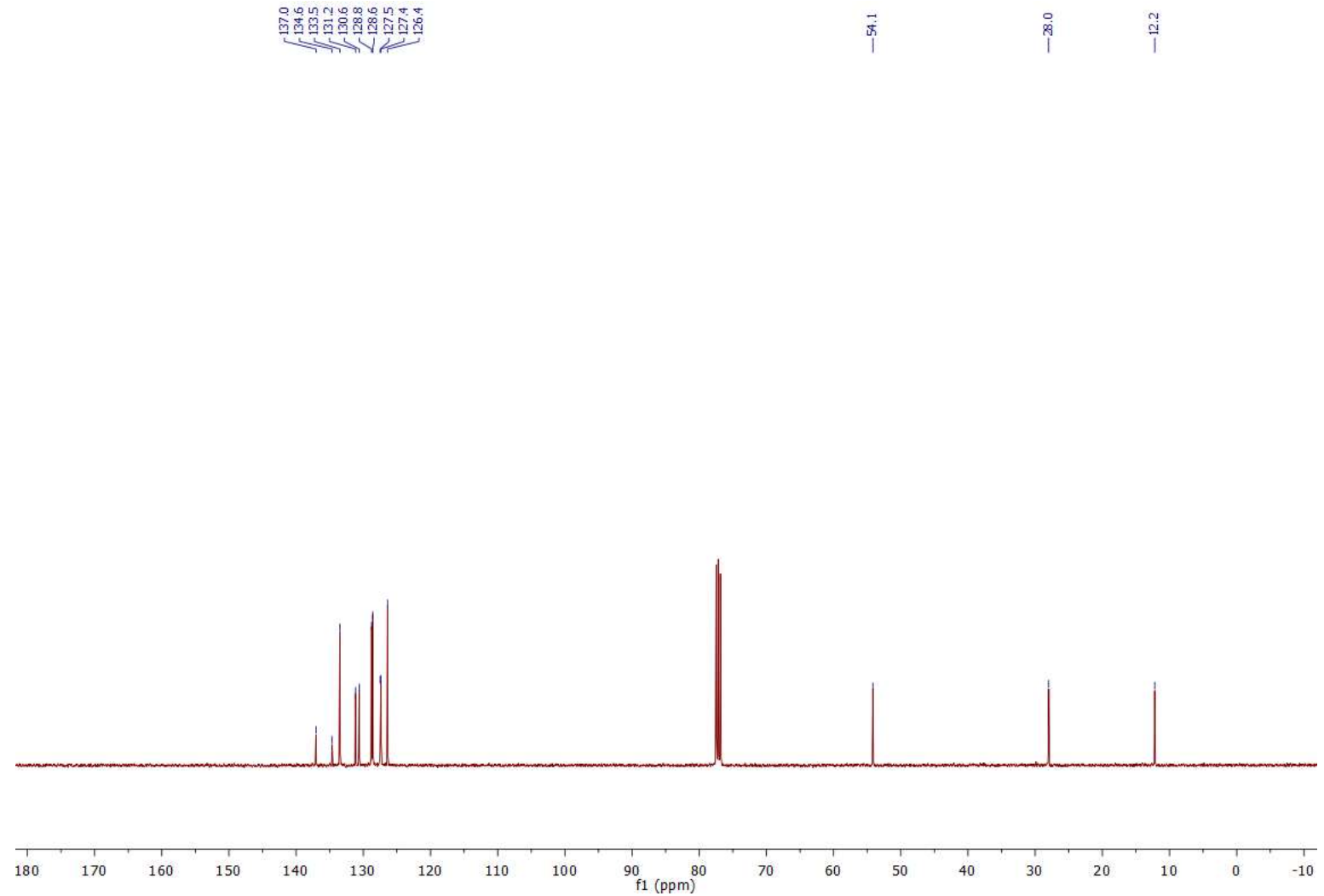
(E)-phenyl(1-phenyloct-1-en-3-yl)sulfane (27)
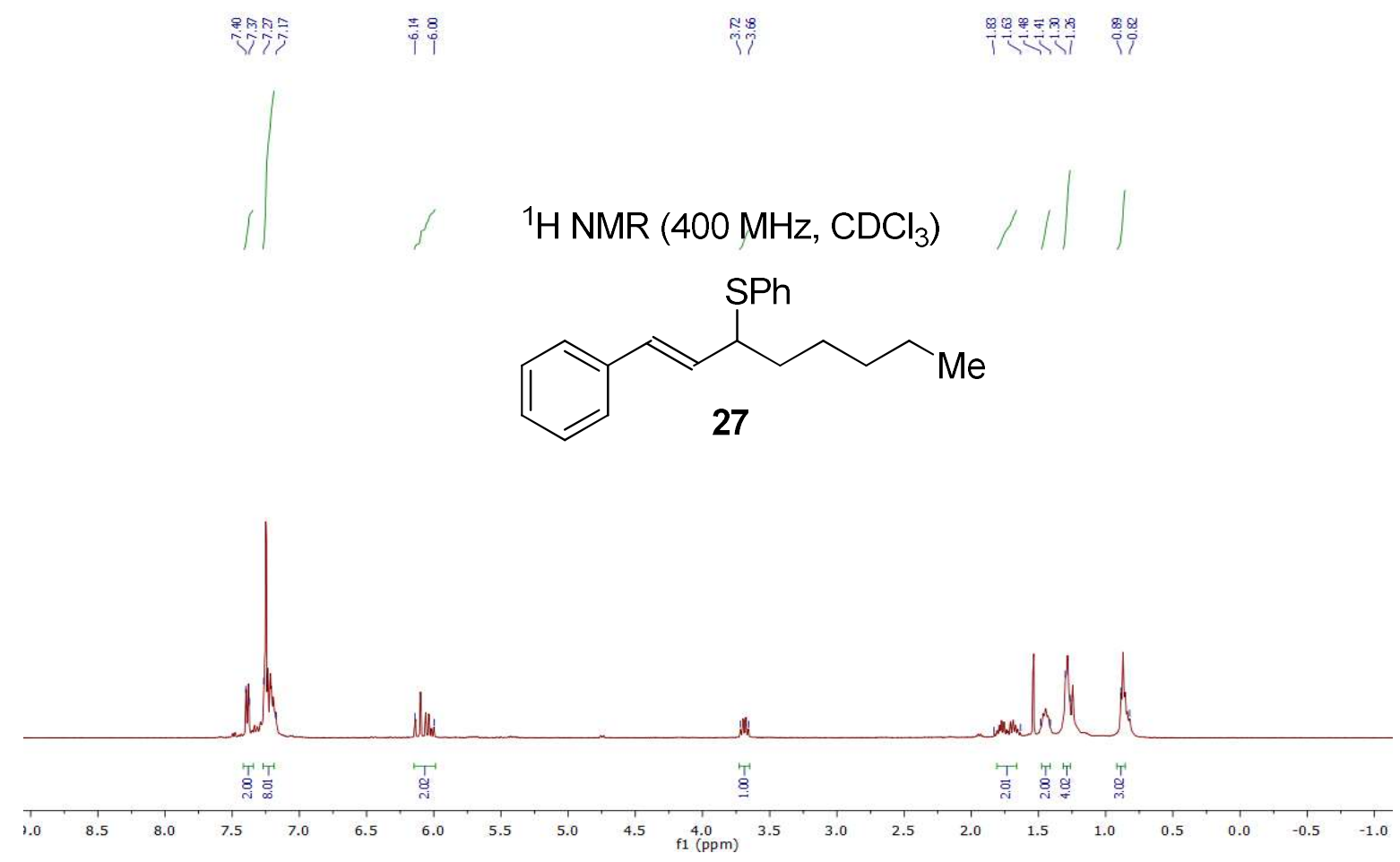

${ }^{13} \mathrm{C}$ NMR $\left(101 \mathrm{MHz}, \mathrm{CDCl}_{3}\right)$

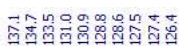

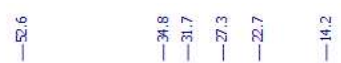

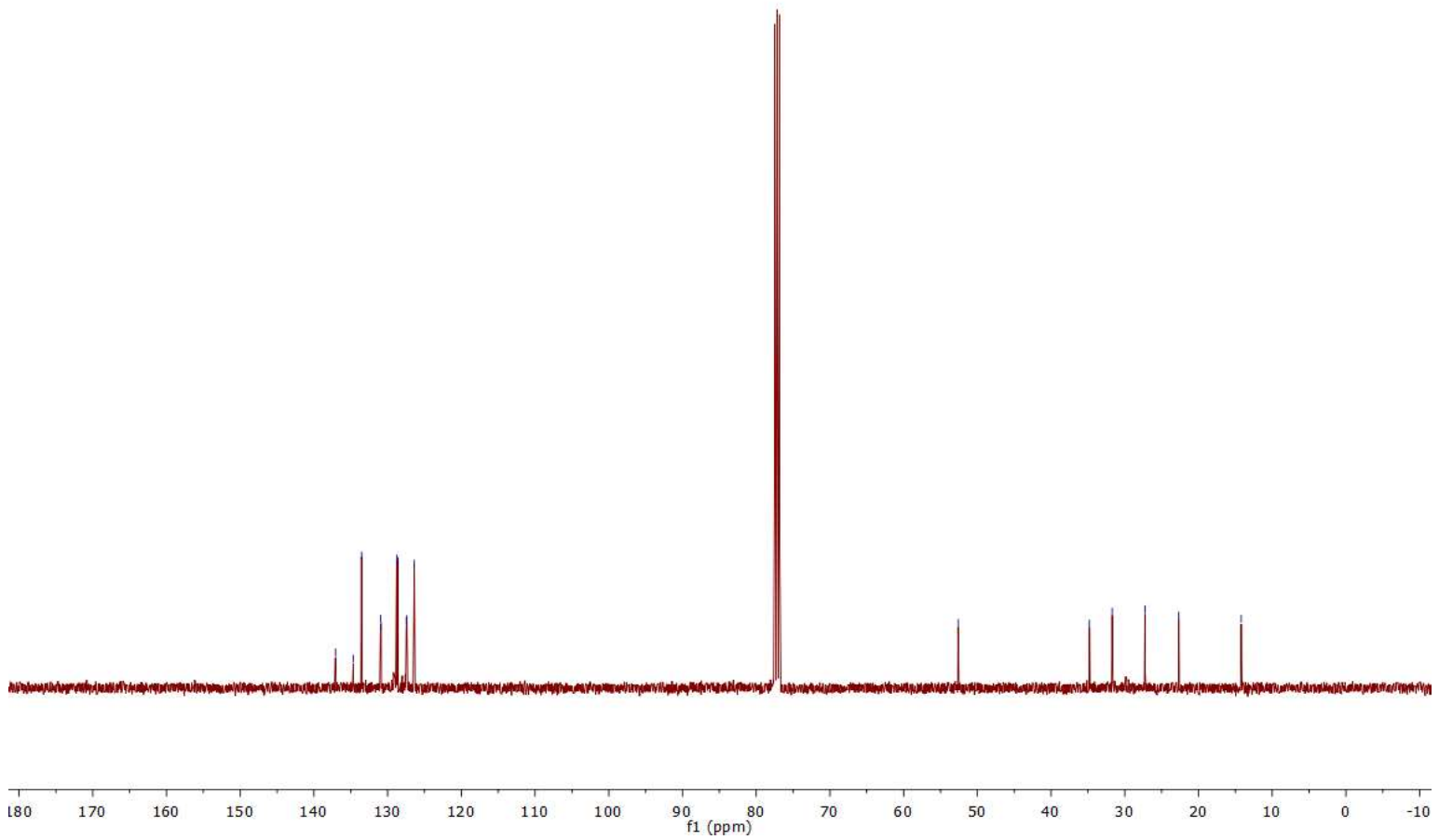


(E)-(2-methyl-4-phenylbut-3-en-2-yl)(phenyl)sulfane (28)

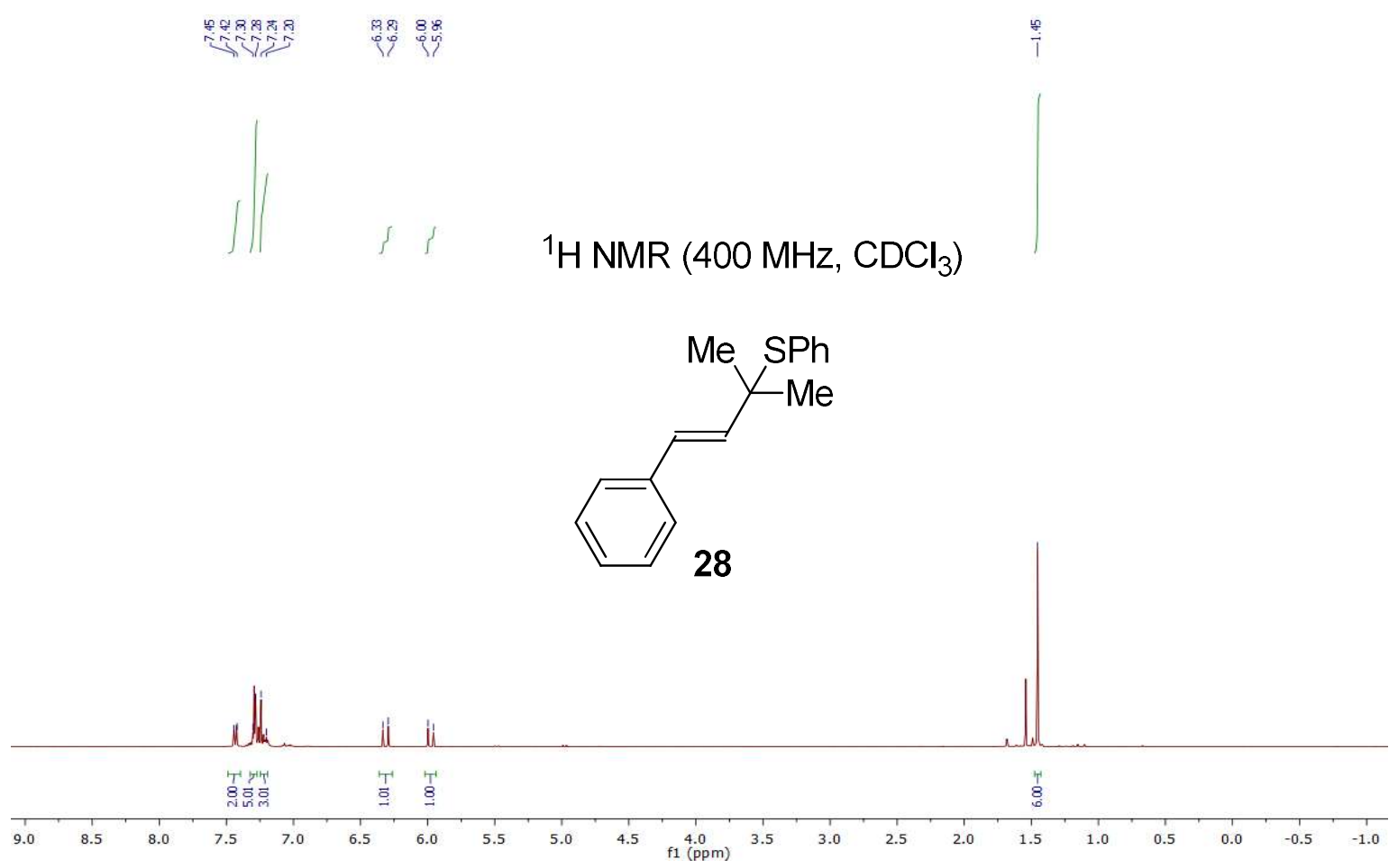

${ }^{13} \mathrm{C}$ NMR $\left(101 \mathrm{MHz}, \mathrm{CDCl}_{3}\right)$

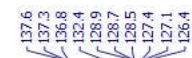

3
1
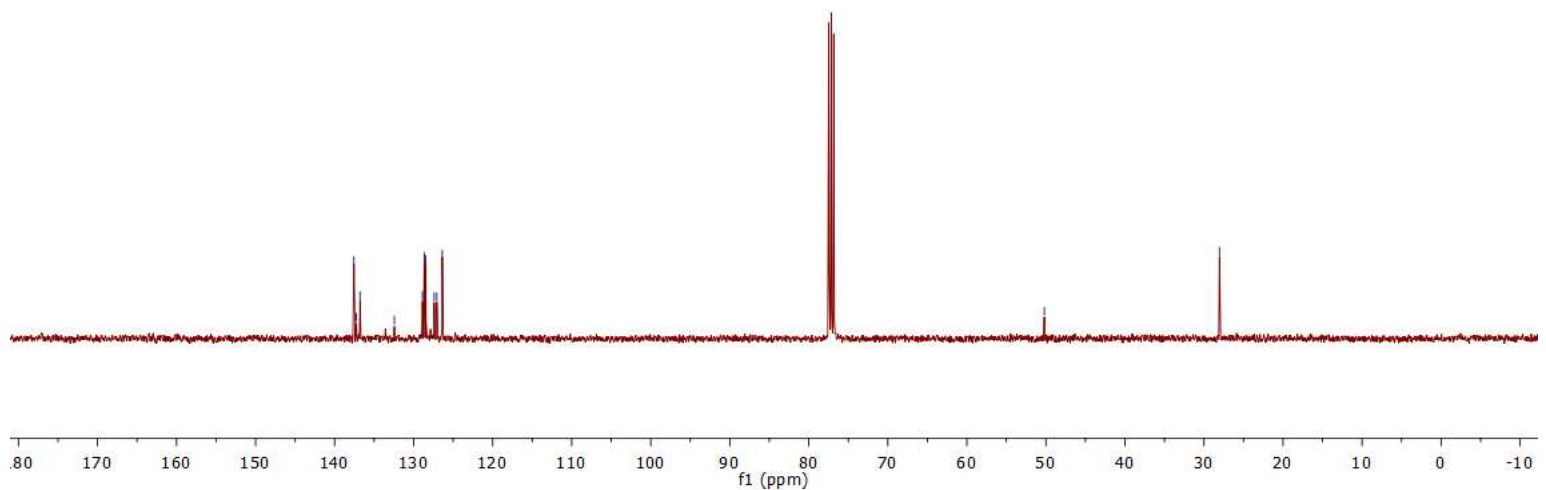
(E)-(3-methyl-1-phenylpent-1-en-3-yl)(phenyl)sulfane (29)
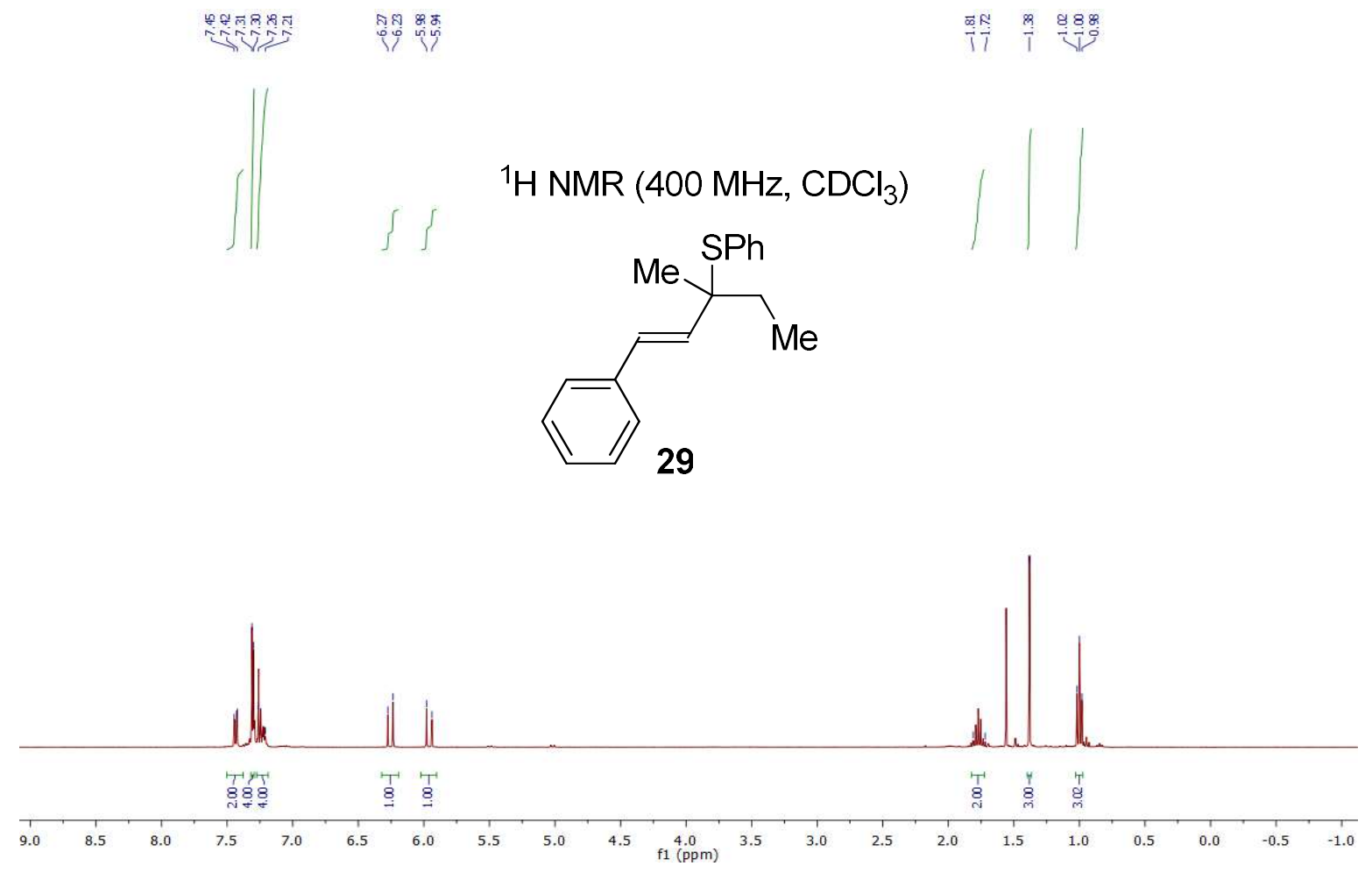

${ }^{13} \mathrm{C}$ NMR $\left(101 \mathrm{MHz}, \mathrm{CDCl}_{3}\right)$
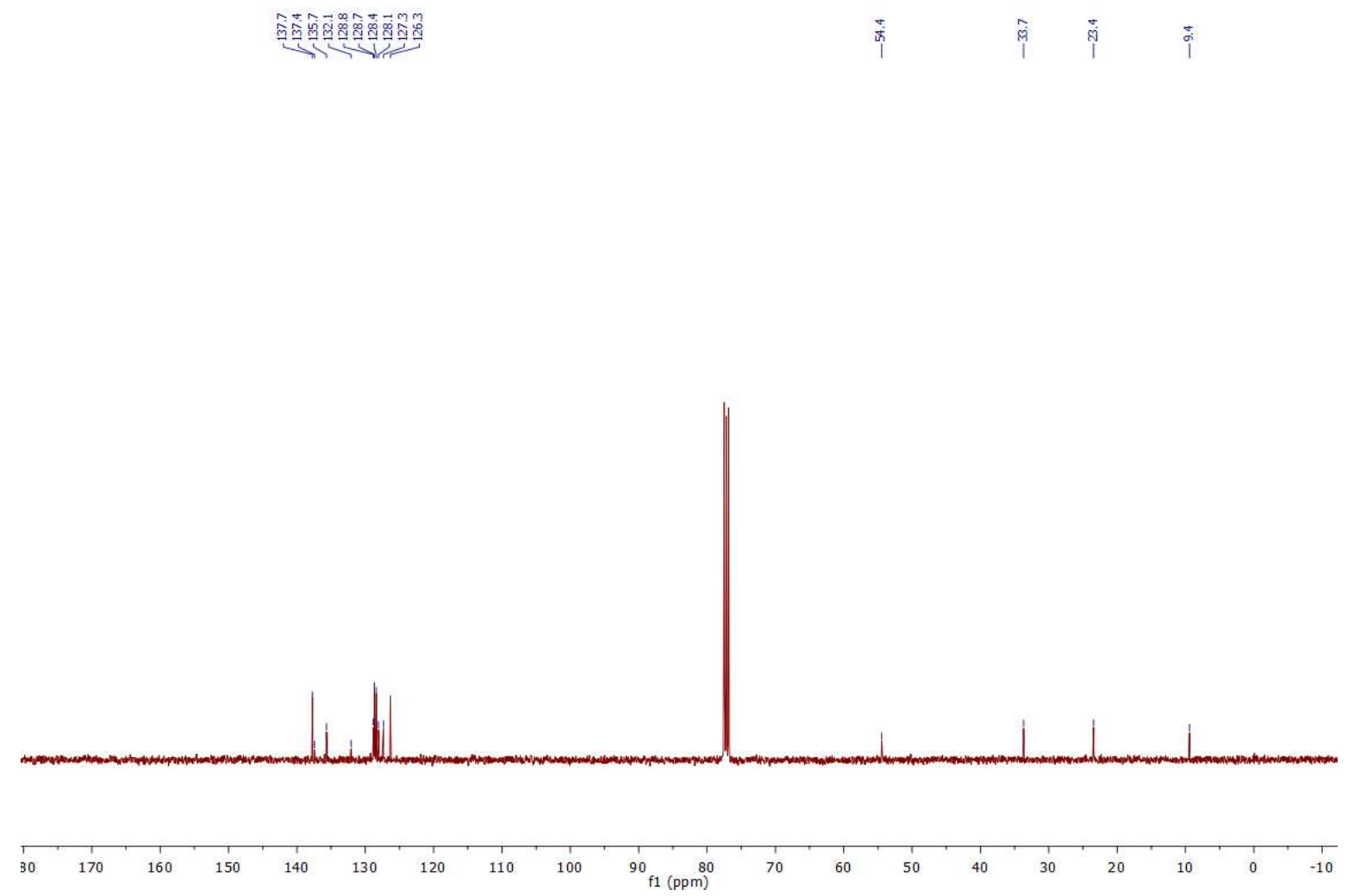
(E)-(3-methyl-1-phenyloct-1-en-3-yl)(phenyl)sulfane (30)
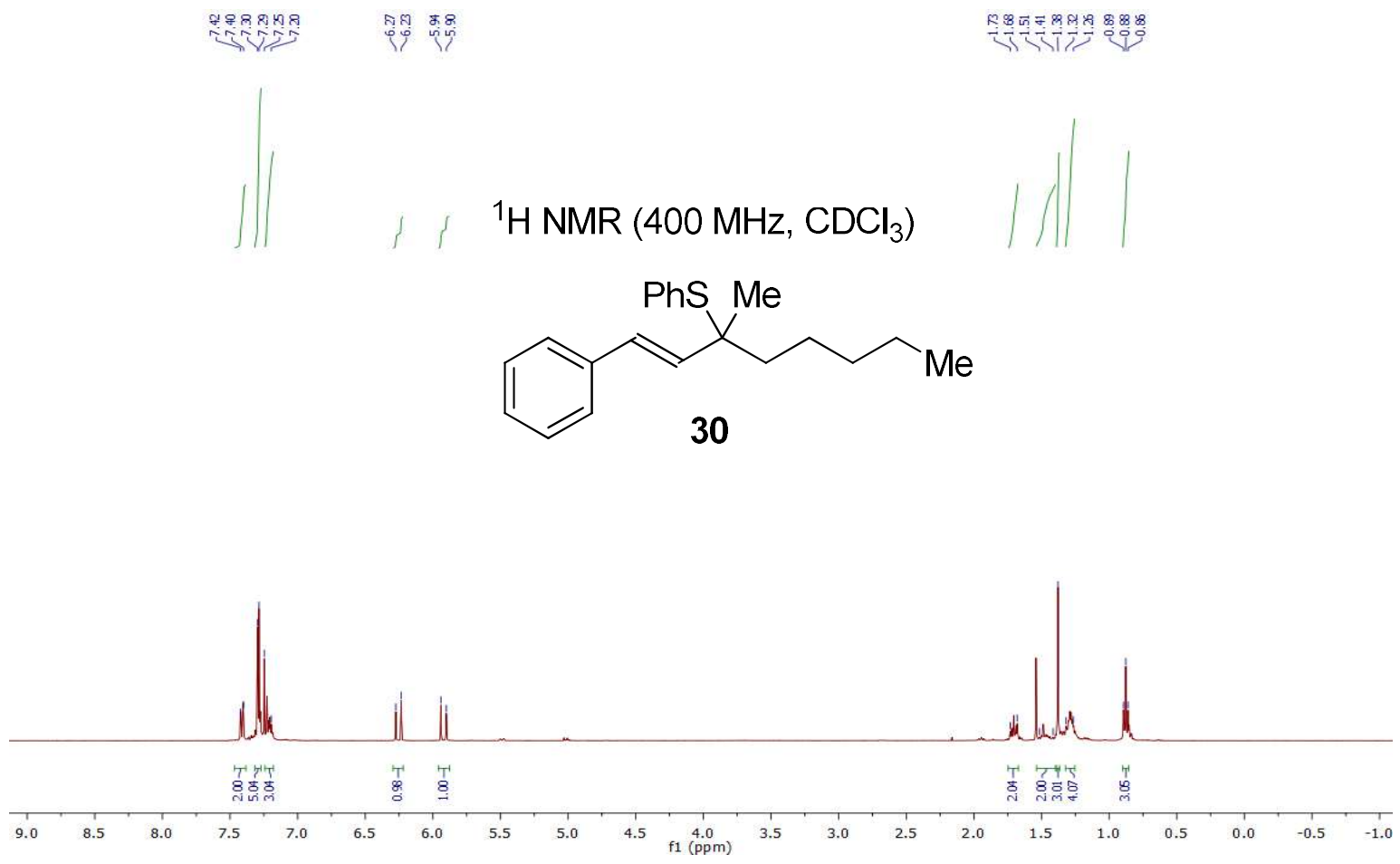

${ }^{13} \mathrm{C}$ NMR $\left(101 \mathrm{MHz} \mathrm{CDCl}_{3}\right)$

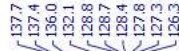

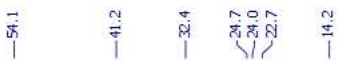

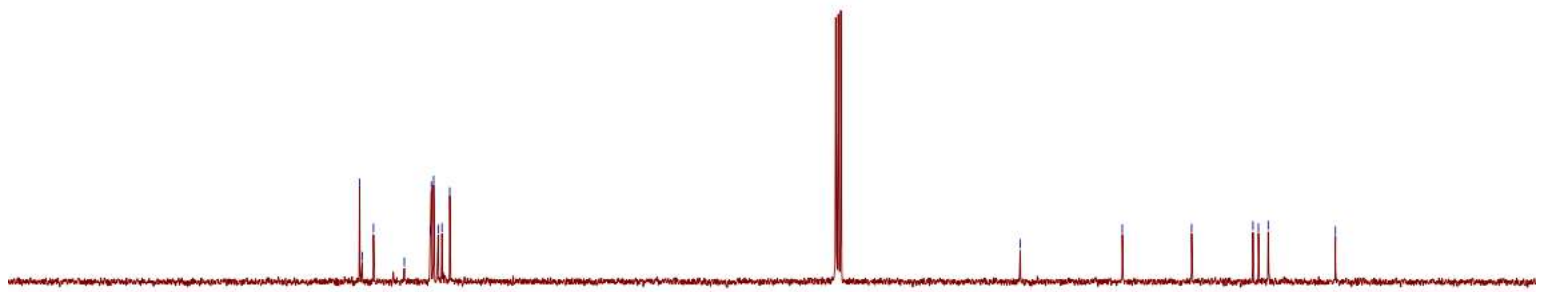

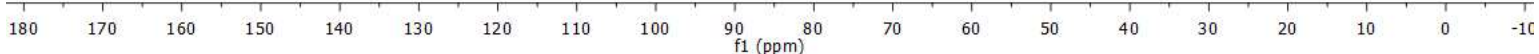


(E)-(2-methyl-1,4-diphenylbut-3-en-2-yl)(phenyl)sulfane (31)
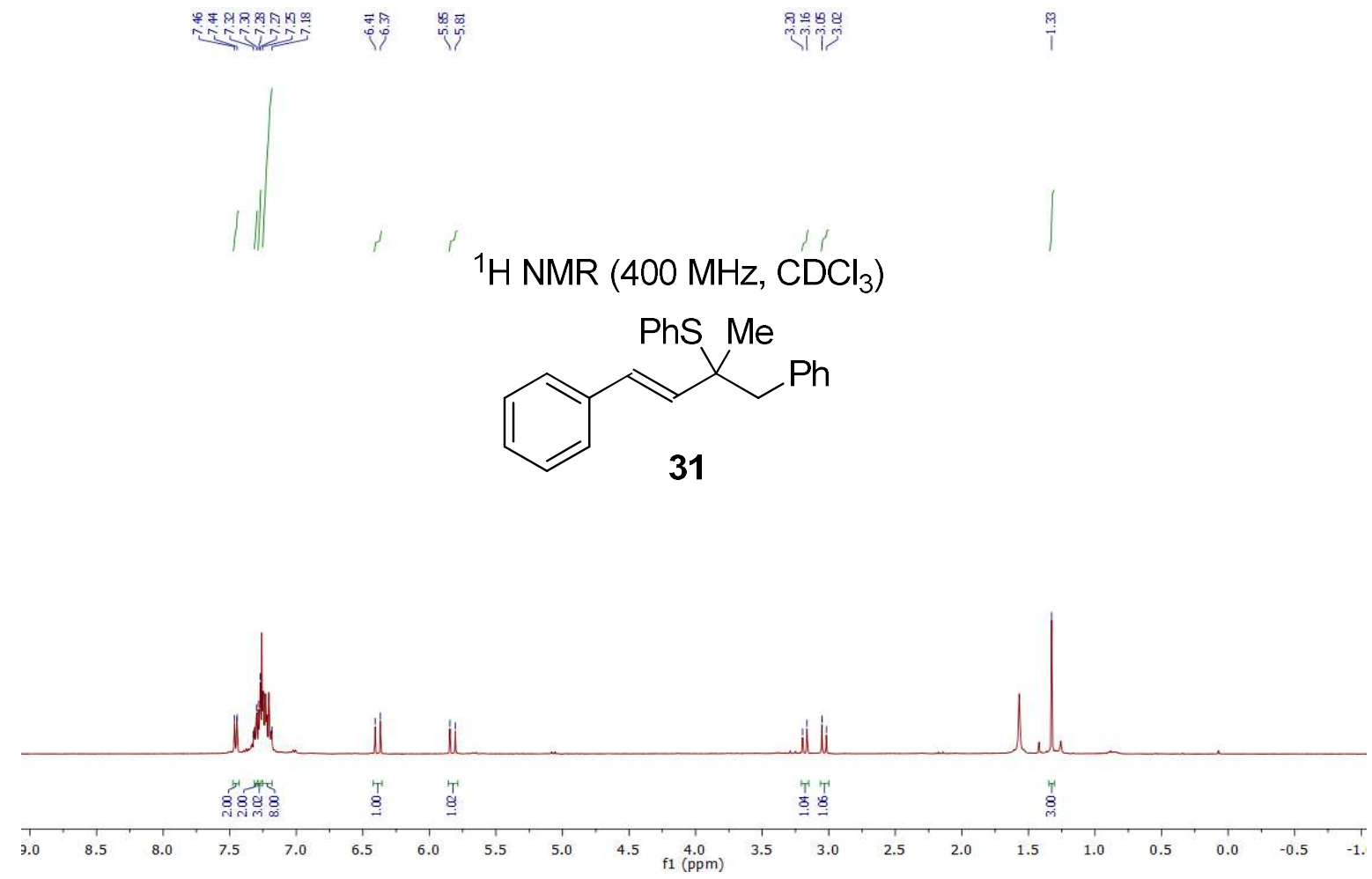

${ }^{13} \mathrm{C}$ NMR $\left(101 \mathrm{MHz}, \mathrm{CDCl}_{3}\right)$

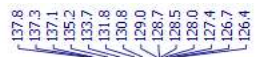

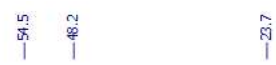
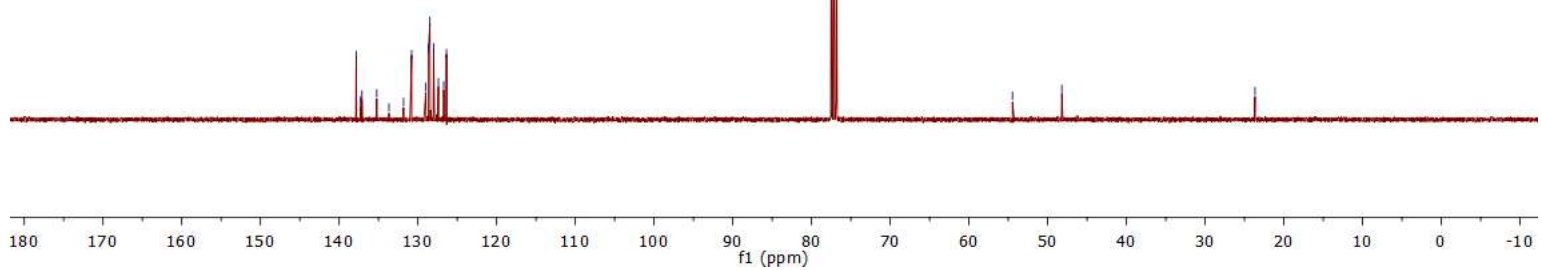
(2-methylbut-2-en-1-yl)(phenyl)sulfane (32)
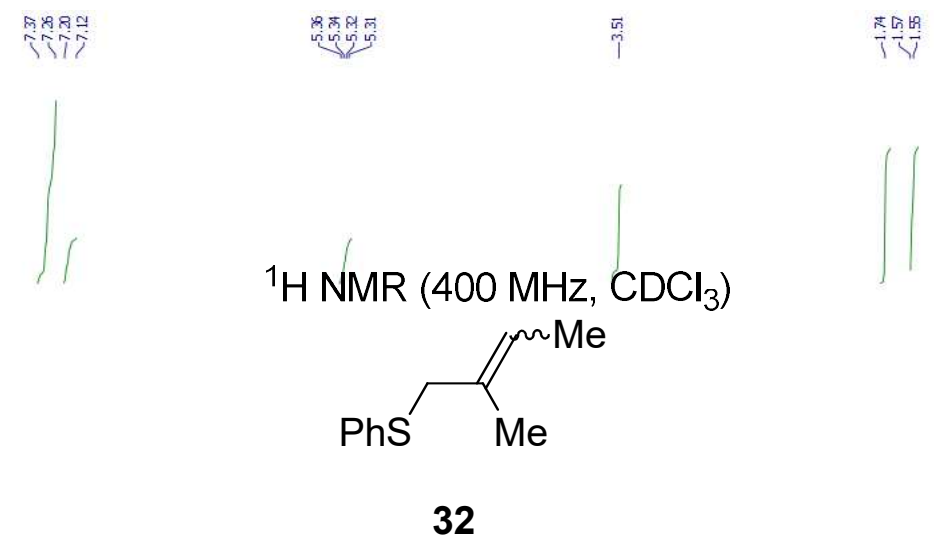

\section{$E / Z=3.6: 1$}

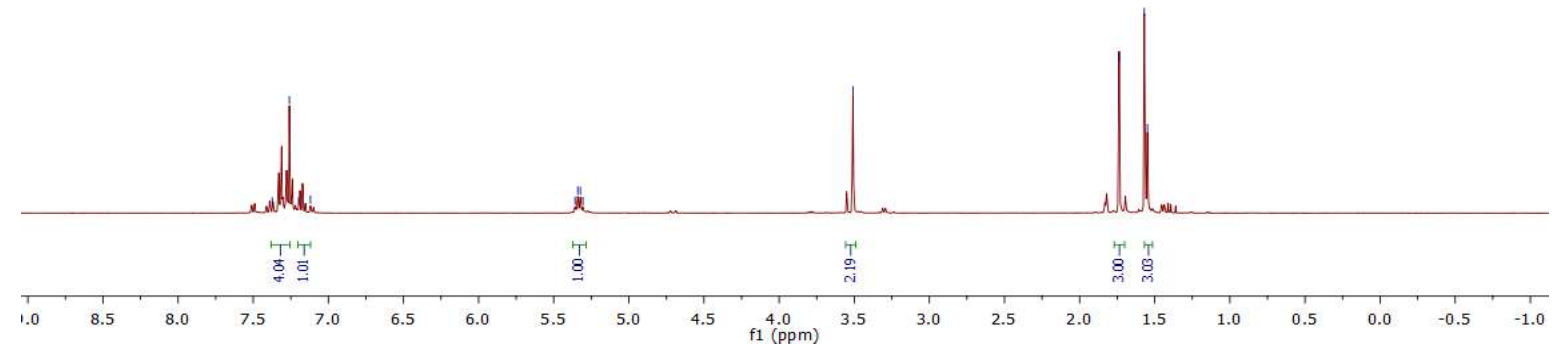

${ }^{13} \mathrm{C}$ NMR $\left(101 \mathrm{MHz}, \mathrm{CDCl}_{3}\right)$
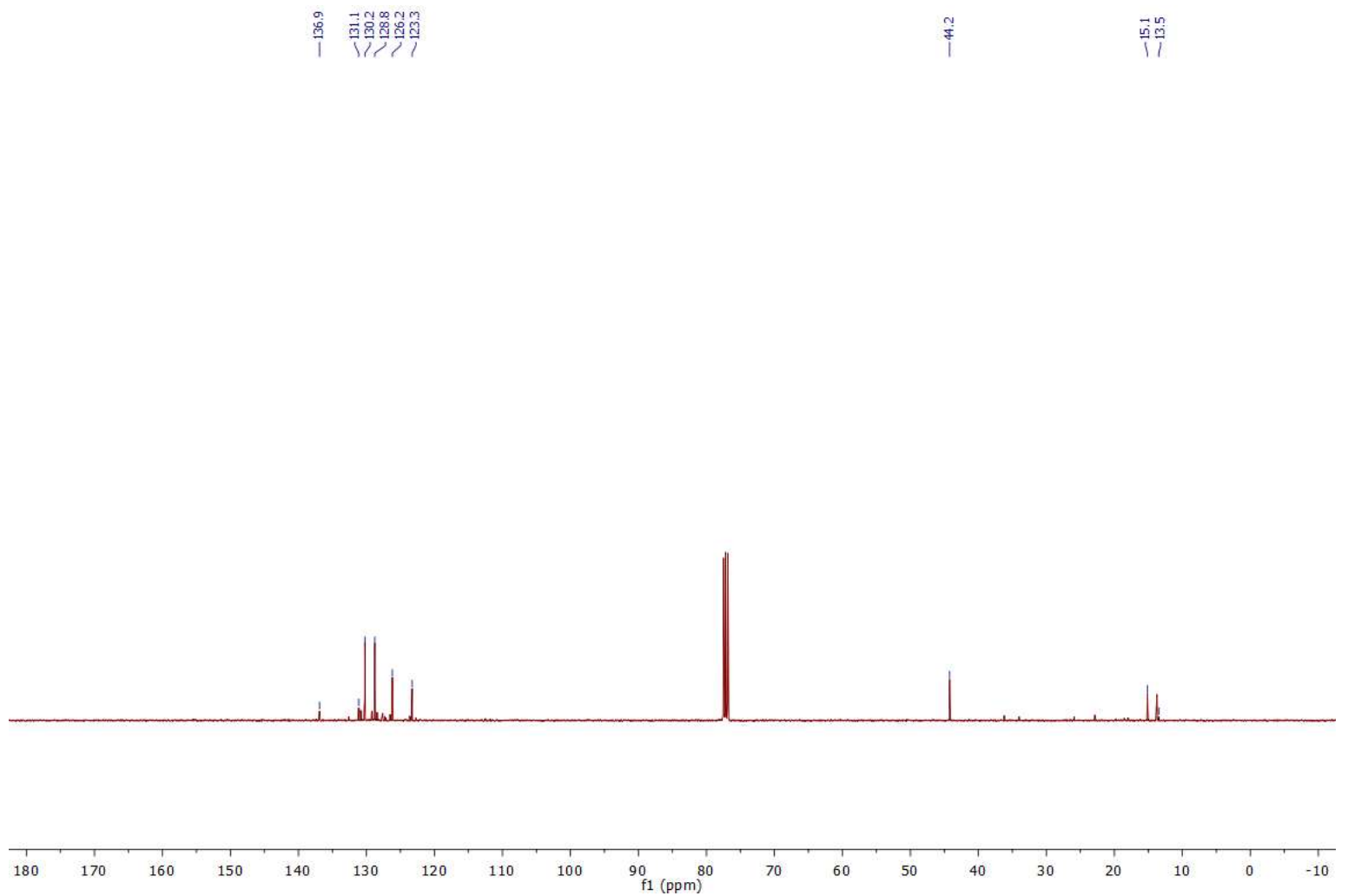


\section{(3-methylbut-2-en-1-yl)(phenyl)sulfane (32')}

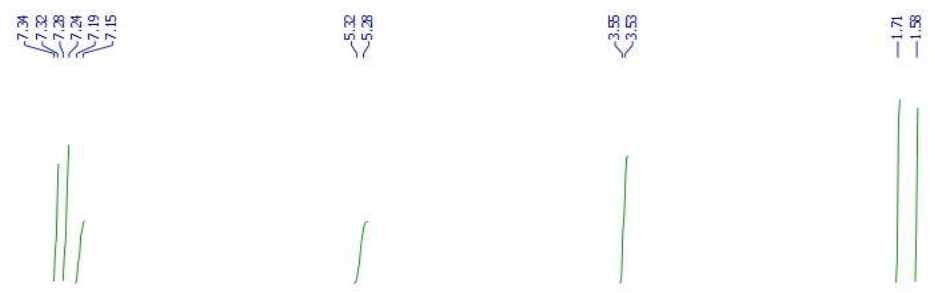

${ }^{1} \mathrm{H}$ NMR (400 MHz, $\mathrm{CDCl}_{3}$ )

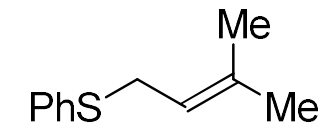

32

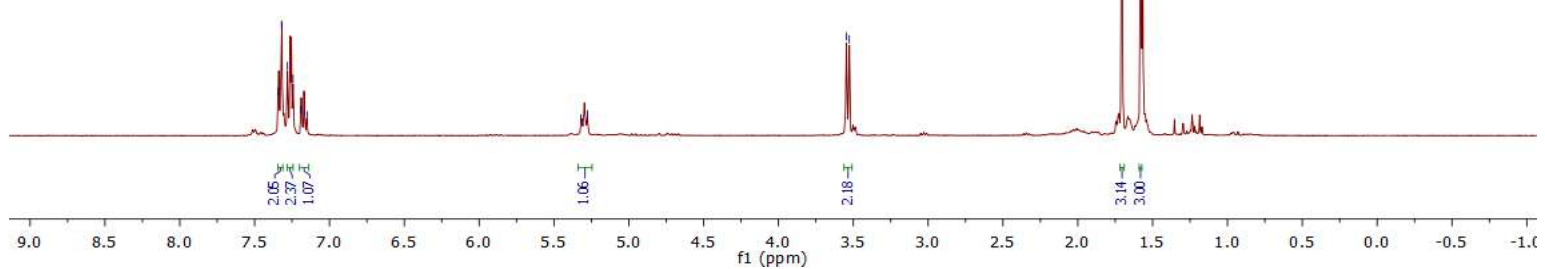

${ }^{13} \mathrm{C}$ NMR $\left(101 \mathrm{MHz}, \mathrm{CDCl}_{3}\right)$
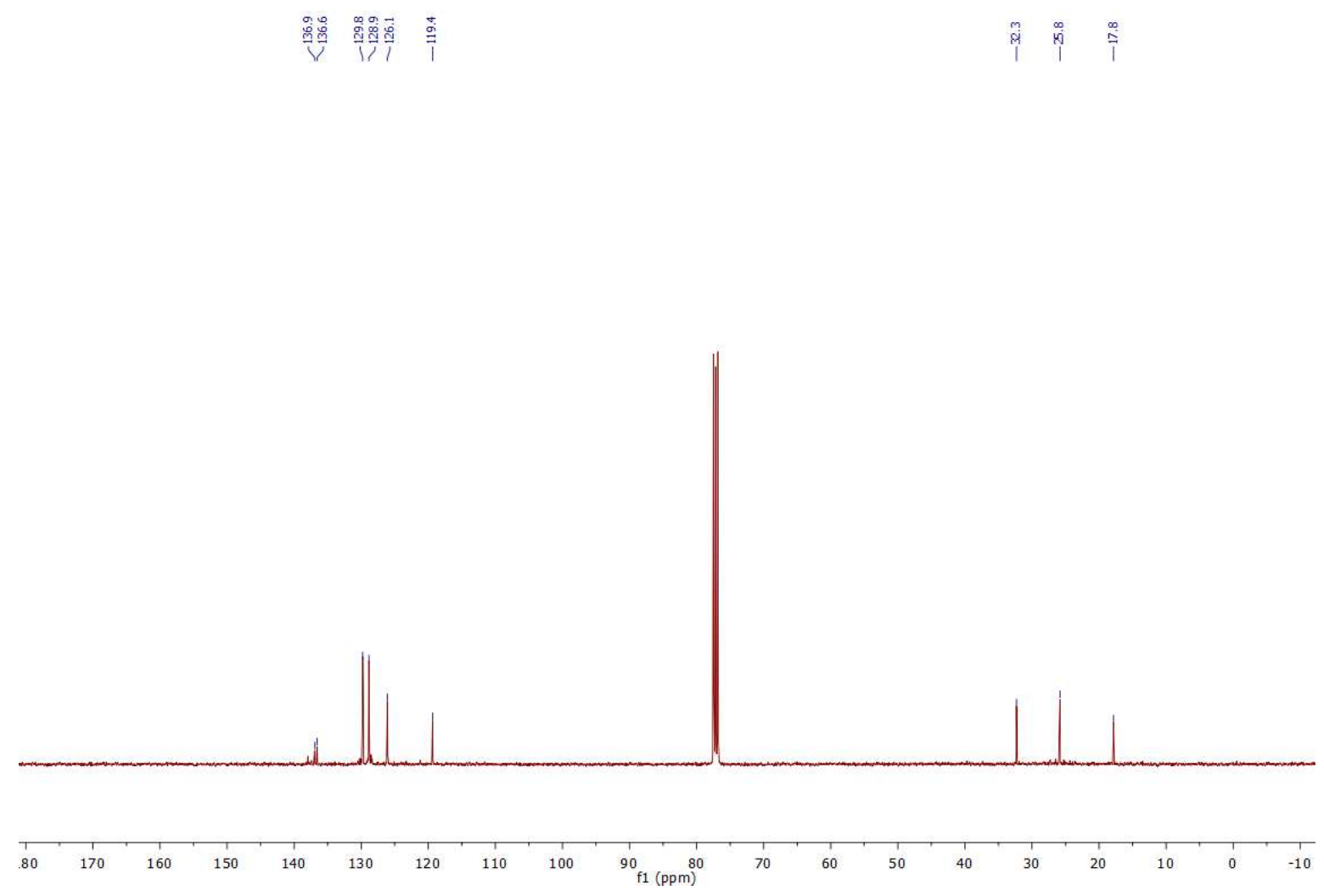
(2-ethylidene-6-methylhept-5-en-1-yl)(phenyl)sulfane (33)
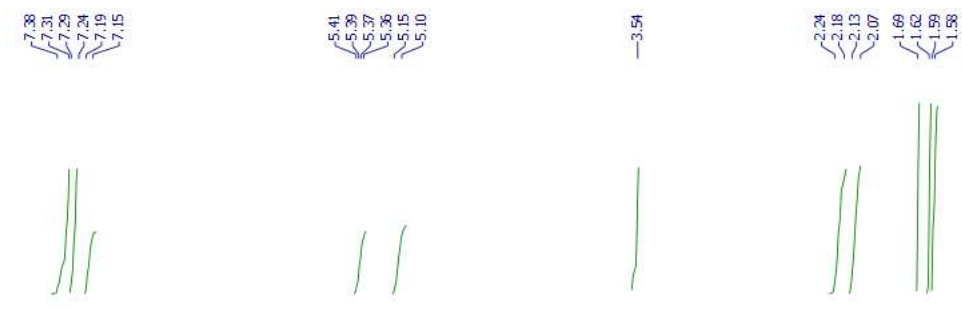

${ }^{1} \mathrm{H}$ NMR (400 $\left.\mathrm{MHz}, \mathrm{CDCl}_{3}\right)$
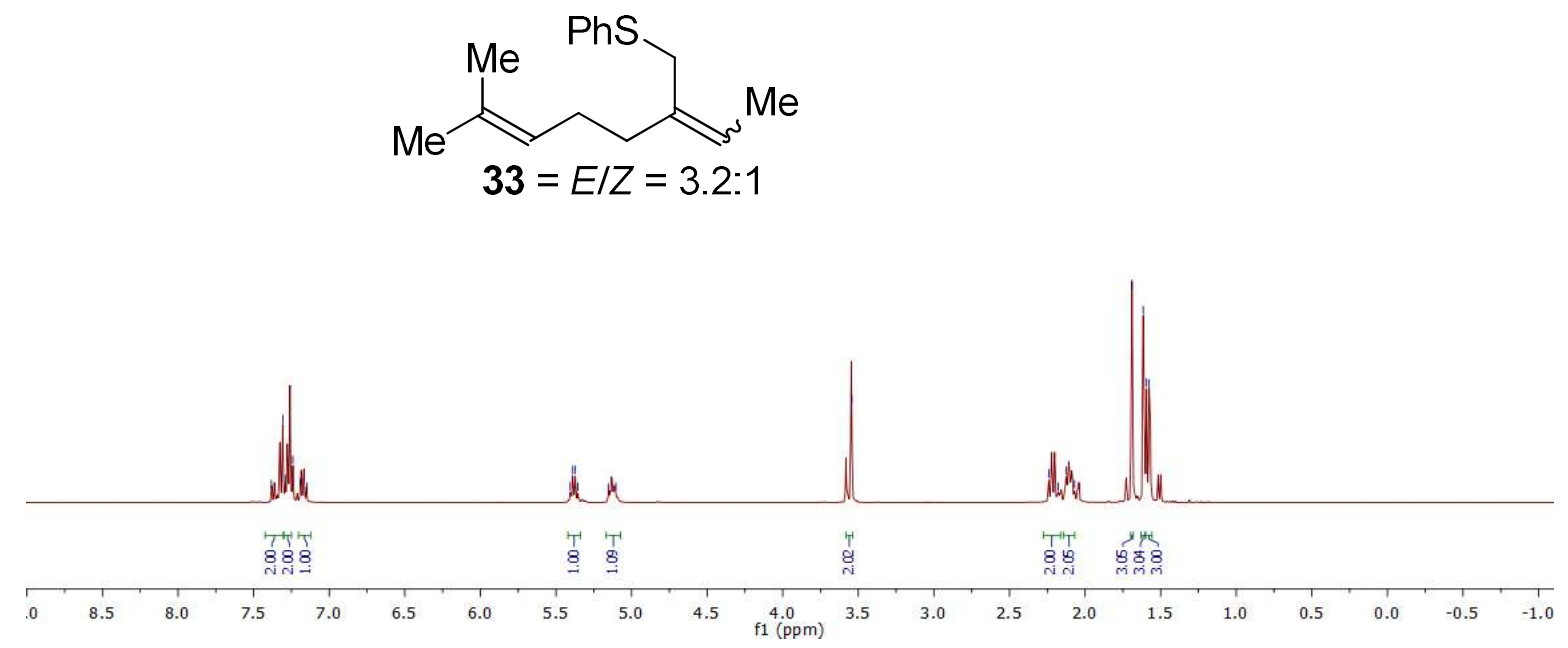

${ }^{13} \mathrm{C}$ NMR $\left(101 \mathrm{MHz}, \mathrm{CDCl}_{3}\right)$

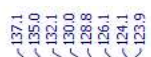

군궈

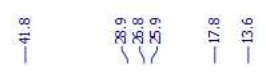
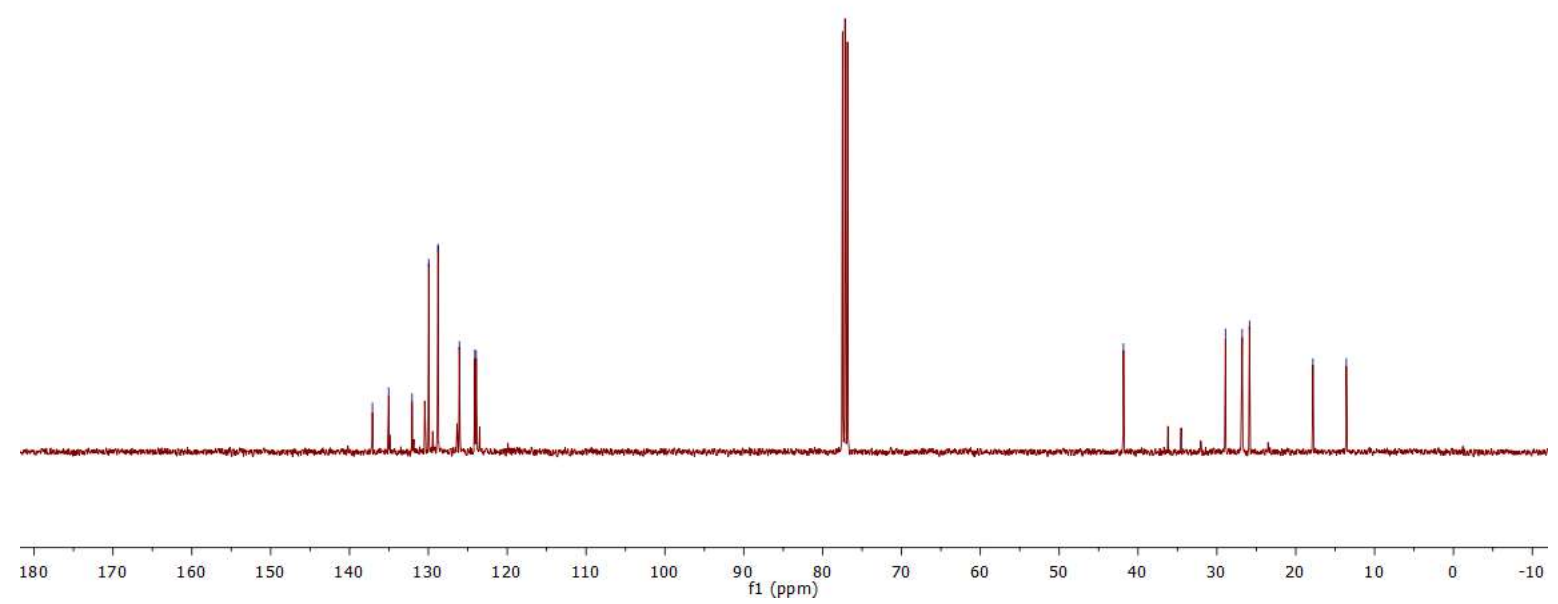


\section{Cyclohex-2-en-1-yl(phenyl)sulfane (34)}

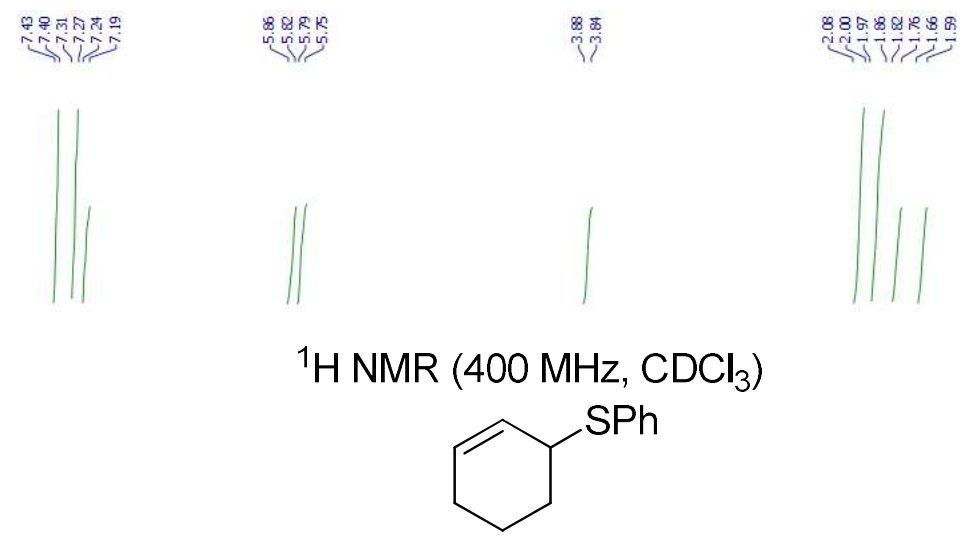

34

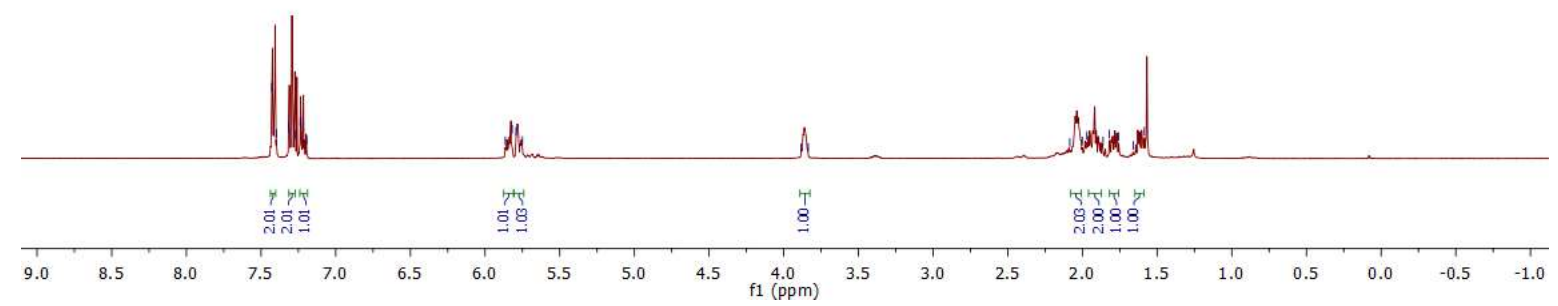

${ }^{13} \mathrm{C}$ NMR $\left(101 \mathrm{MHz}, \mathrm{CDCl}_{3}\right)$

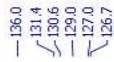

总
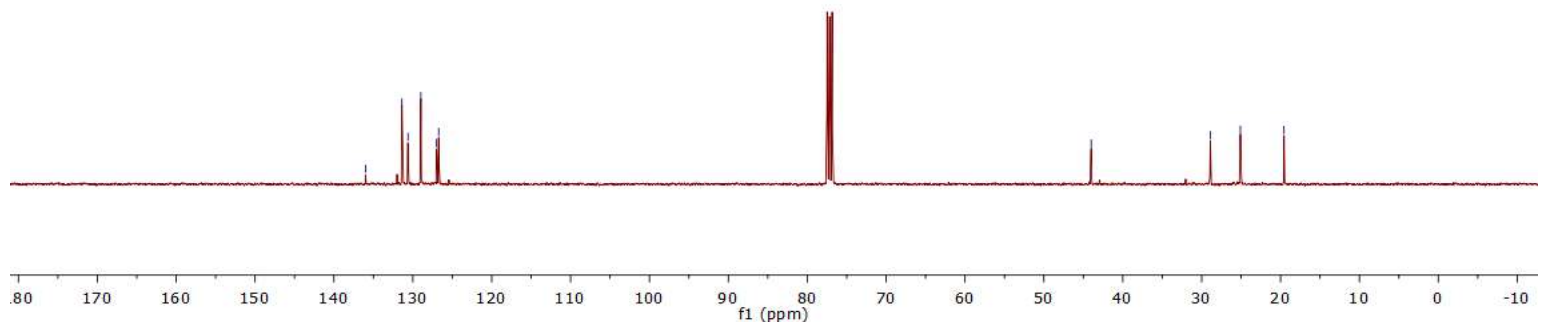
(E)-((4-phenylbut-3-en-2-yl)sulfonyl)benzene (35)

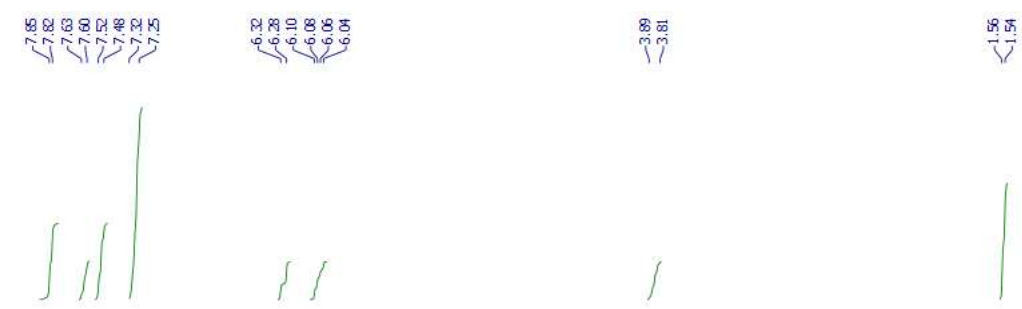

${ }^{1} \mathrm{H}$ NMR (400 MHz, $\left.\mathrm{CDCl}_{3}\right)$<smiles>CC(C=Cc1ccccc1)S(=O)(=O)c1ccccc1</smiles>

35

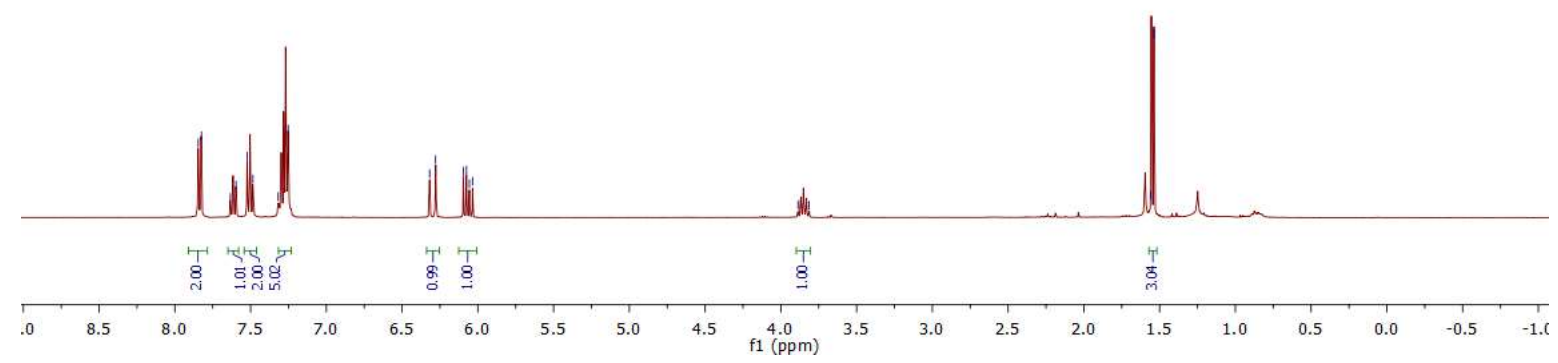

${ }^{13} \mathrm{C}$ NMR $\left(101 \mathrm{MHz}, \mathrm{CDCl}_{3}\right)$
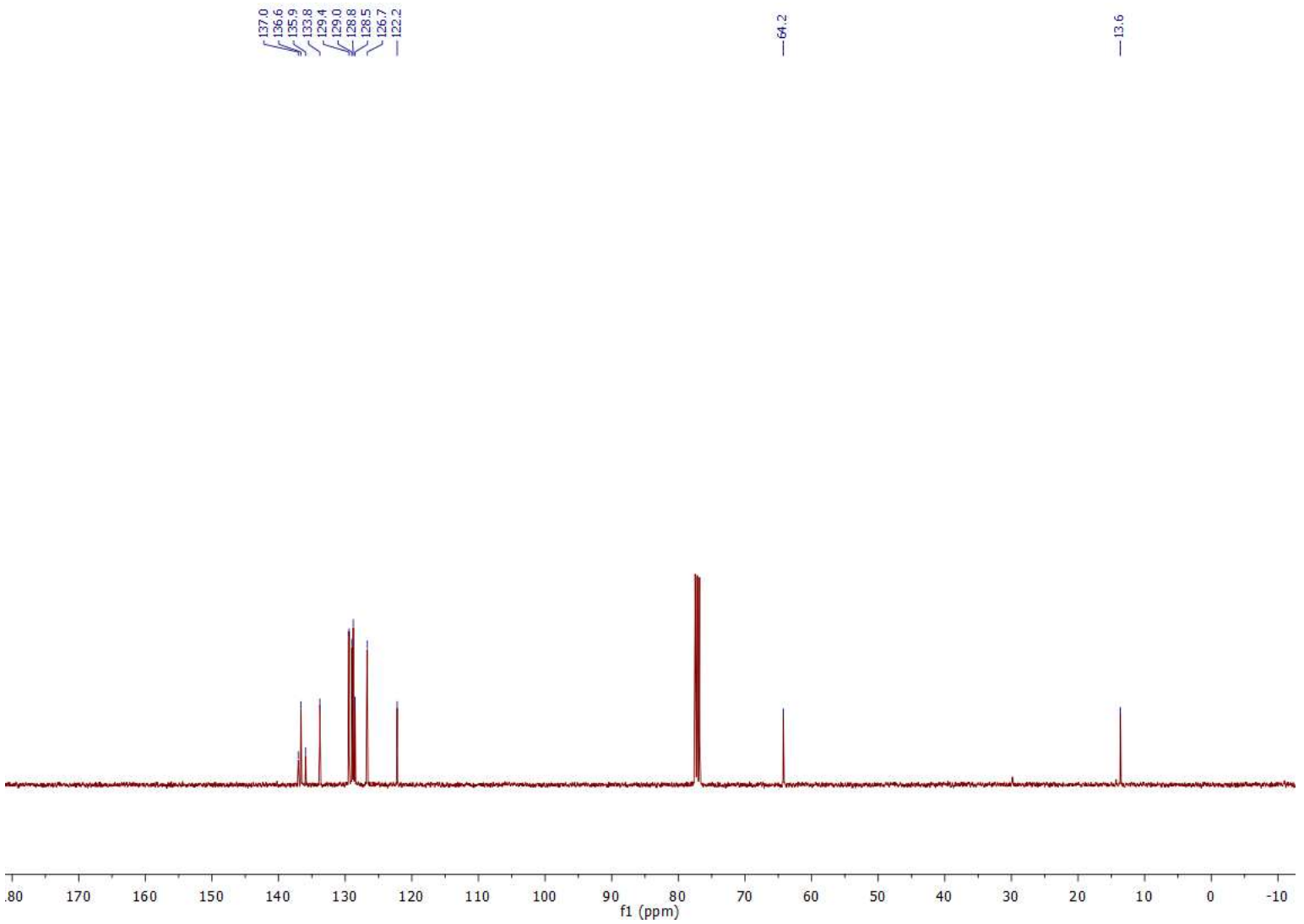
(3-(phenylsulfonyl)but-1-ene-1,1-diyl)dibenzene (36)

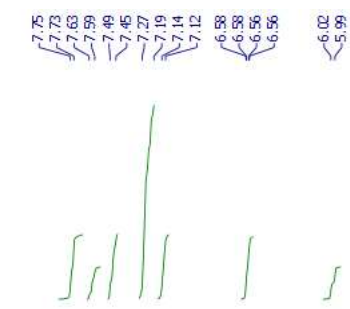

शึ.

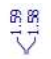

${ }^{1} \mathrm{H} \mathrm{NMR}\left(400 \mathrm{MHz}, \mathrm{CDCl}_{3}\right)$<smiles>CC(C=C(c1ccccc1)c1ccccc1)S(=O)(=O)c1ccccc1</smiles>

36

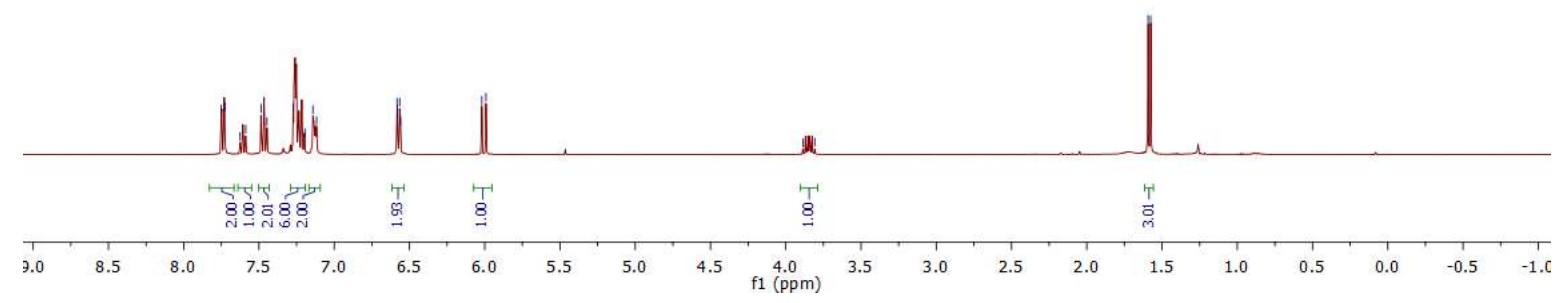

${ }^{13} \mathrm{C}$ NMR $\left(101 \mathrm{MHz}, \mathrm{CDCl}_{3}\right)$
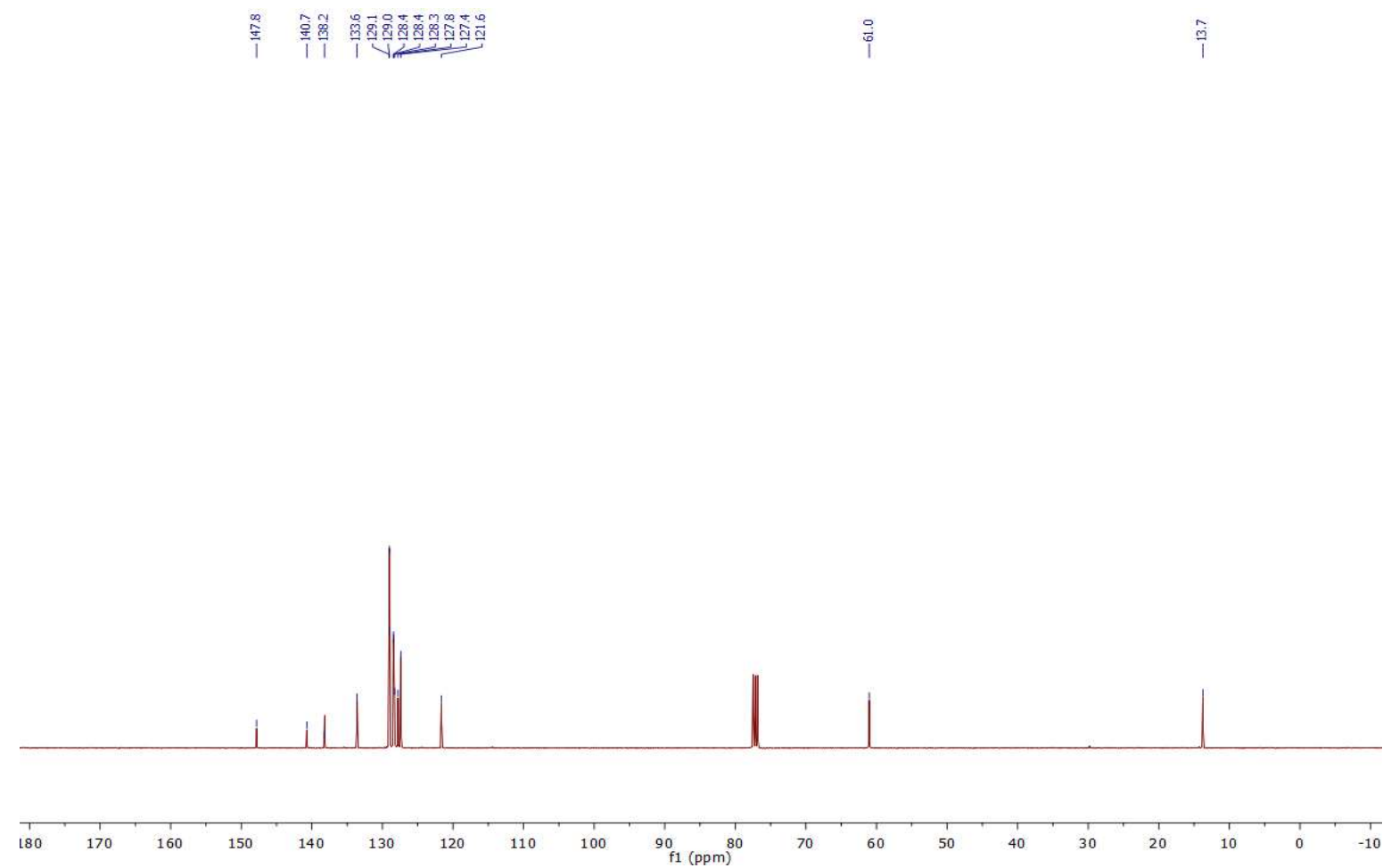
(E)-but-1-en-1-ylbenzene and (E)-but-2-en-1-ylbenzene $\left(37+37^{\prime}\right)$
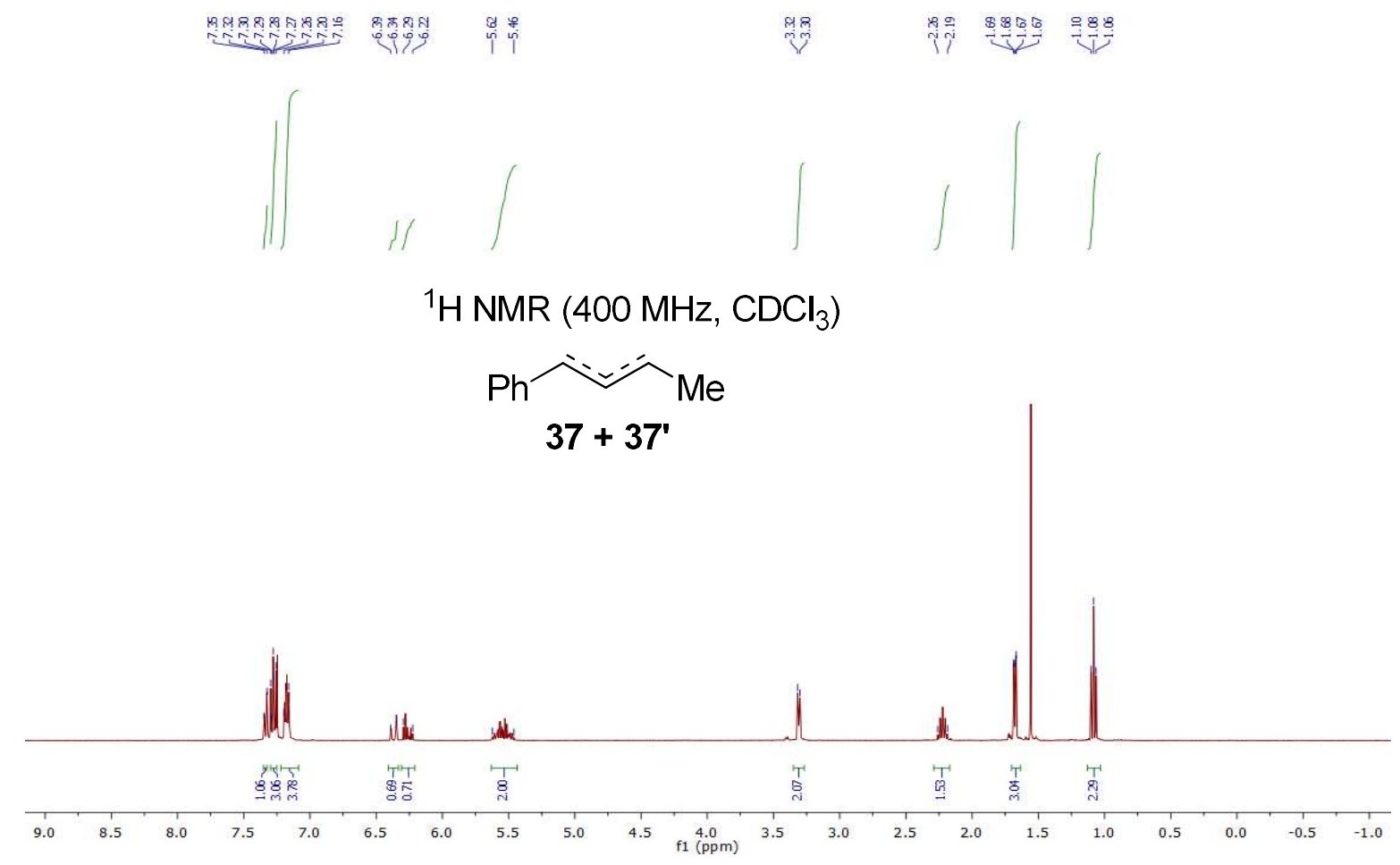

${ }^{13} \mathrm{C}$ NMR $\left(101 \mathrm{MHz}, \mathrm{CDCl}_{3}\right)$

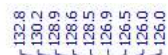

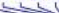
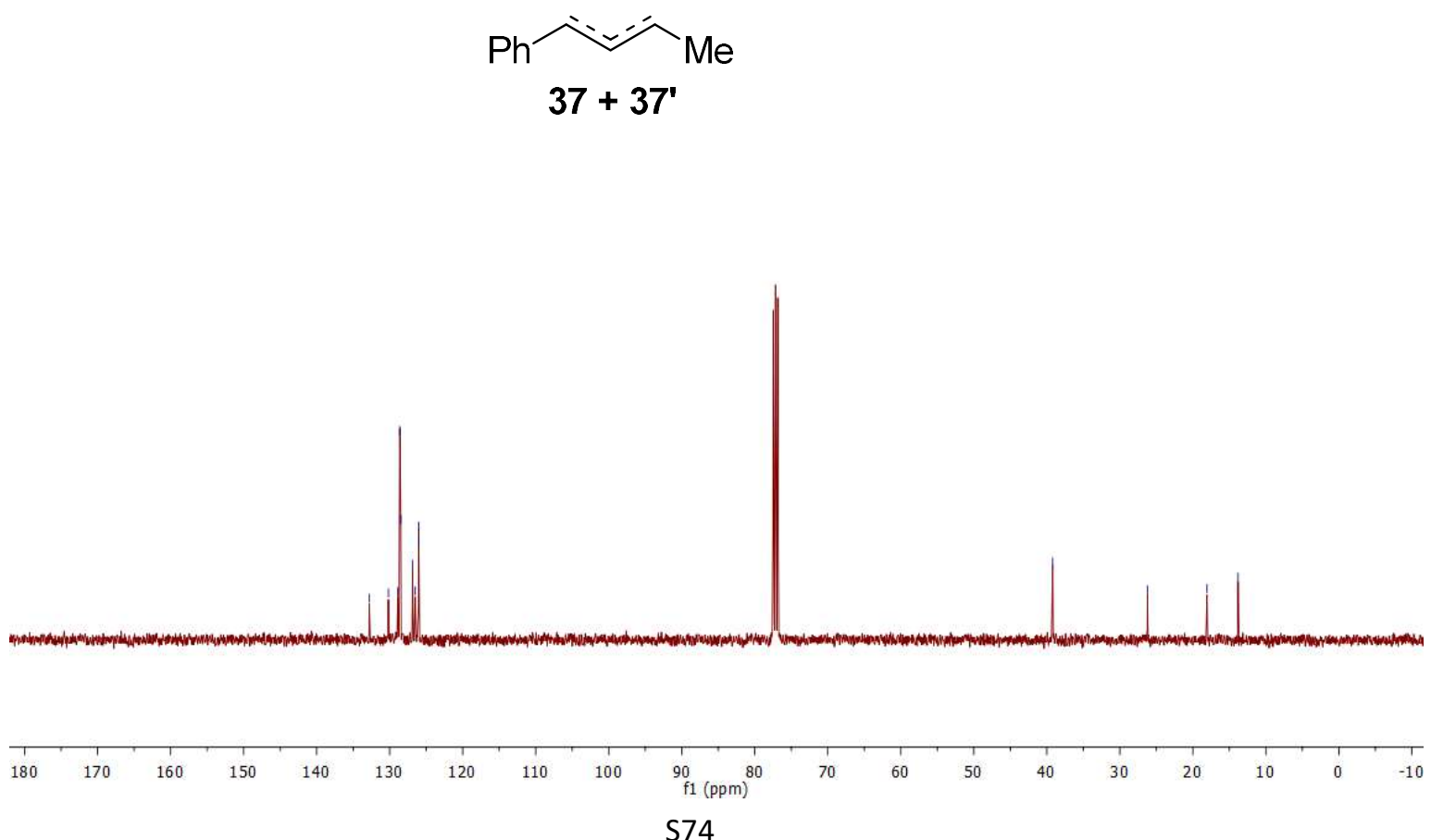


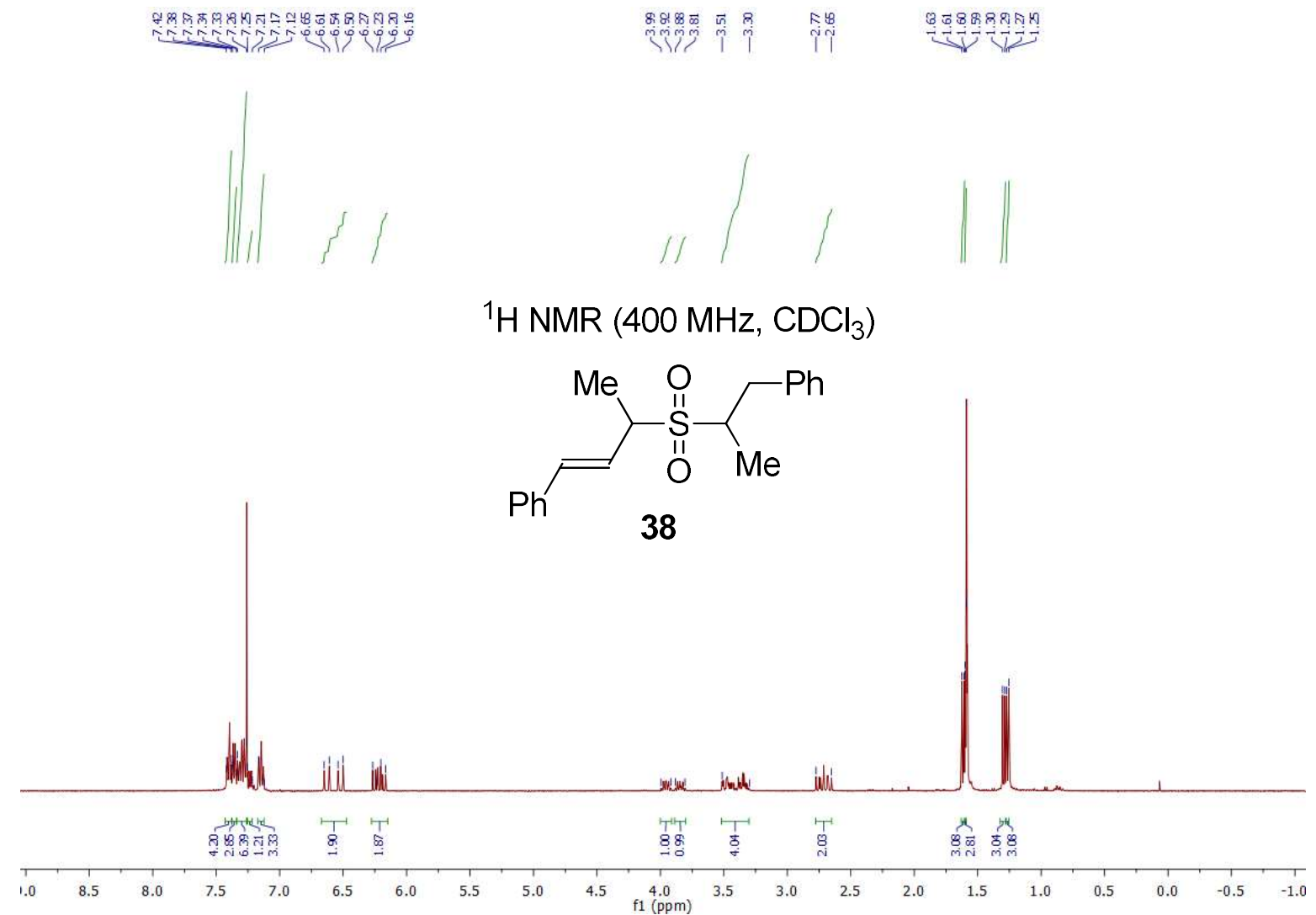

${ }^{13} \mathrm{C}$ NMR $\left(101 \mathrm{MHz}, \mathrm{CDCl}_{3}\right)$

\begin{tabular}{|c|c|}
\hline 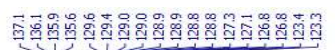 & 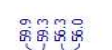 \\
\hline
\end{tabular}
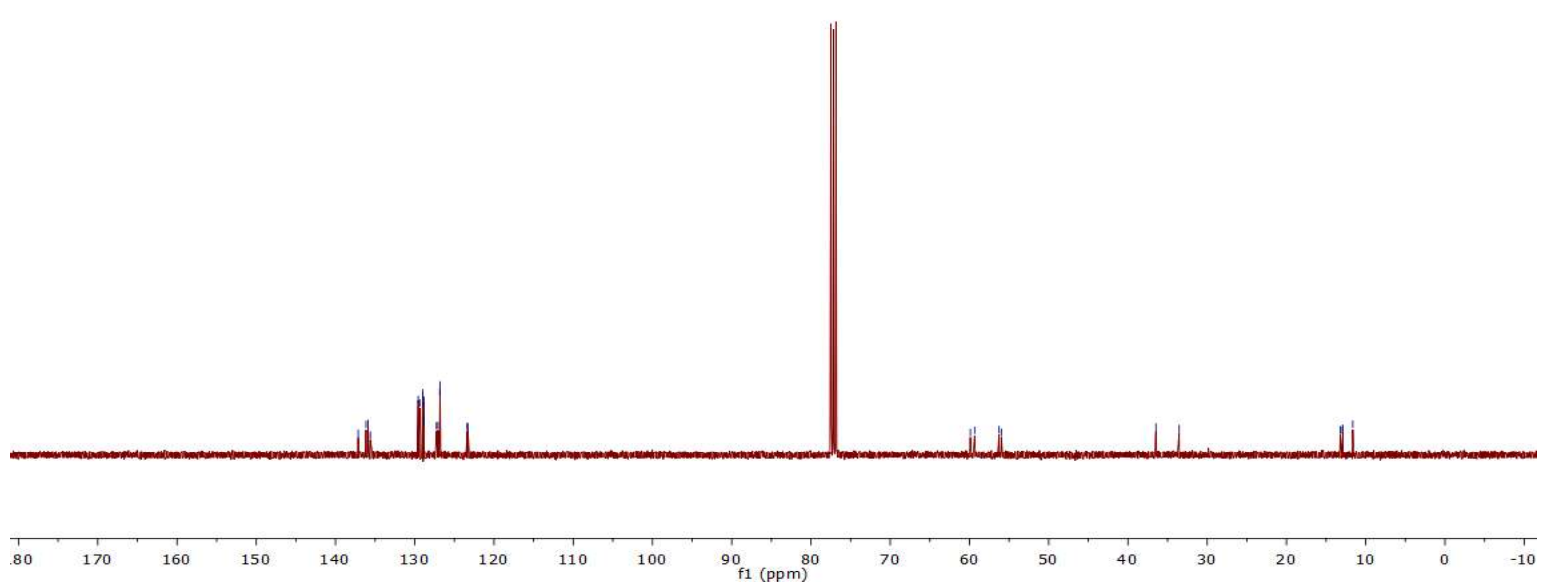
(E)-phenyl(4-phenylbut-3-en-2-yl)sulfane-d $d_{1}$ (3-d)
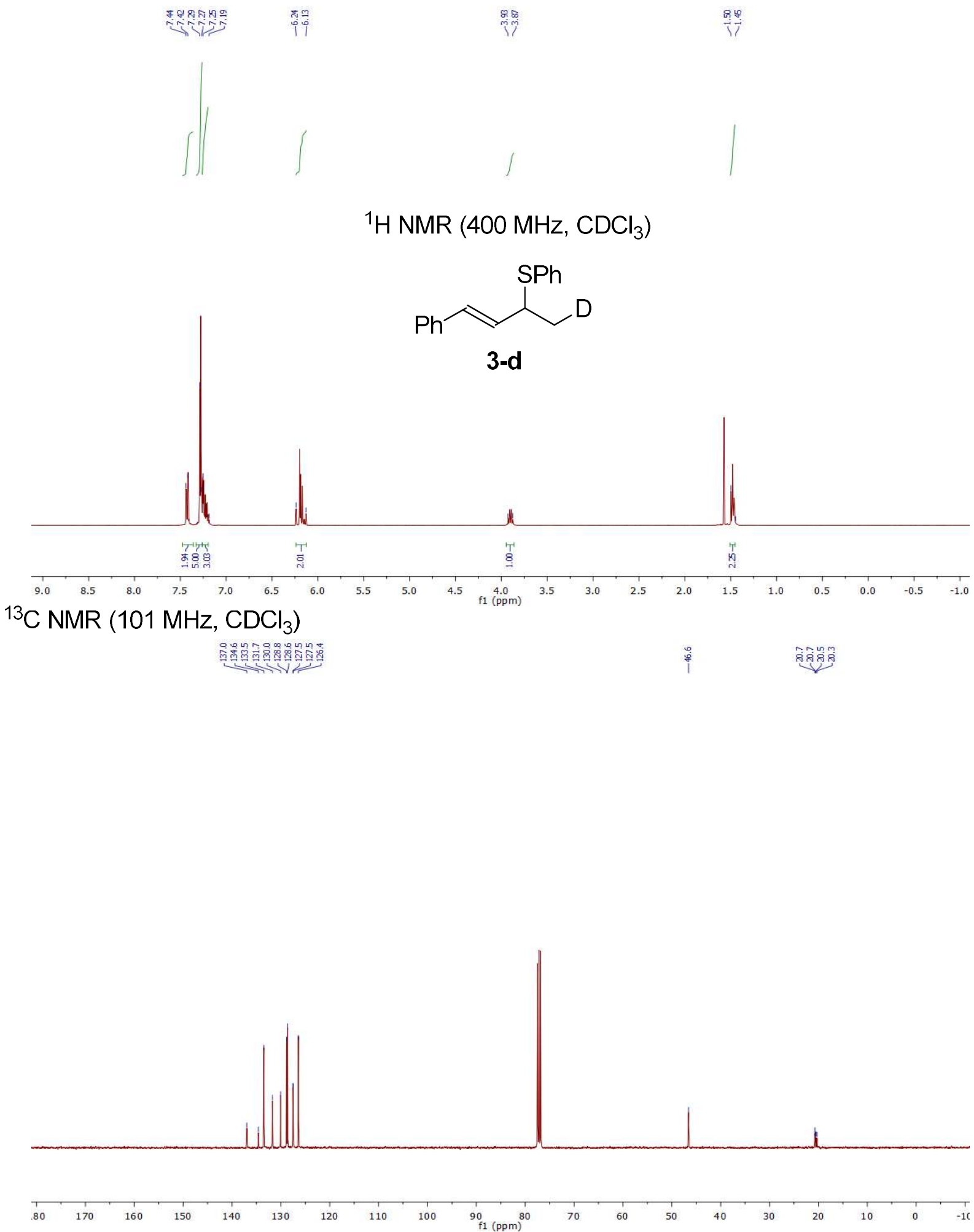
${ }^{13} \mathrm{C}$ DEPT-135 NMR (101 MHz, $\left.\mathrm{CDCl}_{3}\right)$

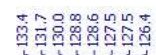

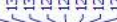

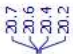

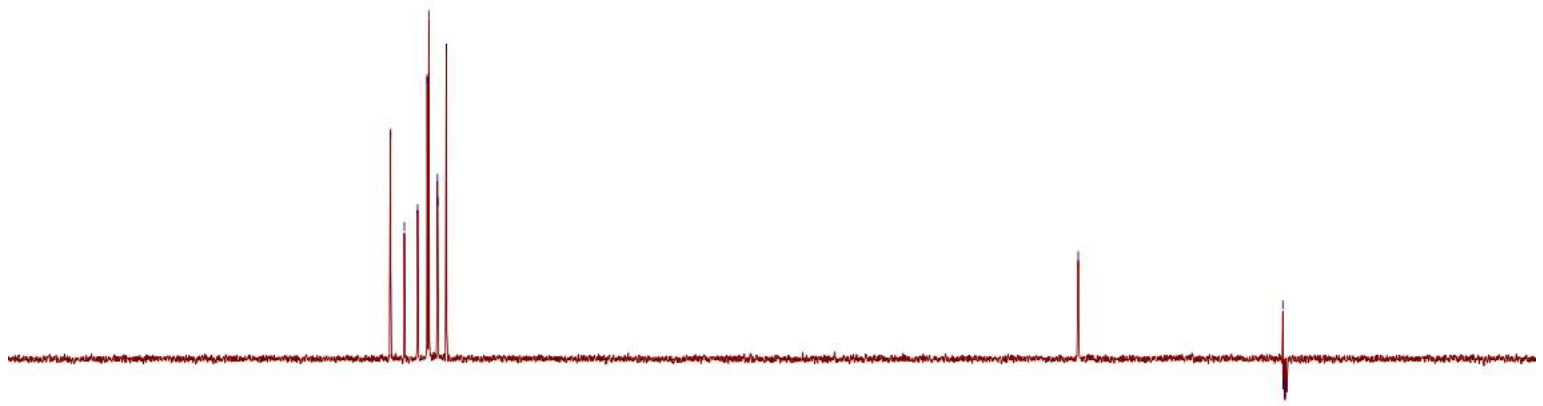

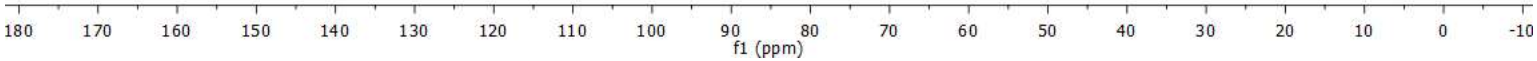




\subsection{DFT calculations}

The following materials are included as supporting information for the DFT calculations parts:

\section{Computational details}

2. Figure S1. The free energy reaction profiles in toluene (in $\mathrm{kcal} / \mathrm{mol}$, at $298 \mathrm{~K}$ and 1 $\mathrm{mol} / \mathrm{L}$ reference concentration) for the $\mathrm{B}\left(\mathrm{C}_{6} \mathrm{~F}_{5}\right)_{3}$-catalyzed hydrothiolation of (A) 1phenyl 1,3-diene 1a and (B) 1-methyl 1,3-diene $\mathbf{m} 1$ using thiophenol 2a, computed at the PW6B95-D3/def2-QZVP + COSMO-RS // TPSS-D3/def2-P + COSMO level of theory. (C) DFT-supported intramolecular Ph...allyl cation interaction model $\left(\mathrm{pBa}^{+}\right)$ that may enhance the obseverved site 1 protonation.

3. Table S2. TPSS-D3/def2-TZVP + COSMO computed lowest imaginary frequency $(\mathrm{ImF})$, zero-point energies (ZPE), gas-phase enthalpic $(\mathrm{Hc})$ and Gibbs free-energy (Gc) corrections; the COSMO-RS computed solvation enthalpic (Hsol) and Gibbs free-energy (Gsol) corrections in toluene solution; TPSS-D3/def2-QZVP and PW6B95-D3/def2-QZVP single-point energies (TPSS-D3 and PW6B95-D3); the relative electronic energies ( $\triangle \mathrm{ET}$ and $\triangle \mathrm{EP}$ ) and Gibbs free-energies ( $\triangle \mathrm{GT}$ and $\Delta \mathrm{GP}$ ) at the TPSS-D3 and PW6B95-D3 levels. Each structure is labeled either by its molecular formula or a specific name in bold. Transition structures (with only one imaginary frequency) are indicated by the "TS" prefix. See Figure S1 for structural labellings.

4. Table S3. The TPSS-D3/def2-TZVP + COSMO optimized atomic Cartesian coordinates (in $\AA$ ) in toluene. Each structure is labeled by the specific name (See also Figure $\mathbf{S 1}$ and Table S2), followed by the number of atoms, the total energy, and the detailed atomic coordinates.

14.1 Computational Details: The quantum chemical DFT calculations have been performed with the TURBOMOLE 7.3 suite of programs ${ }^{[12]}$ The structures are fully optimized at the TPSS-D3/def2-TZVP + COSMO(Toluene) level of theory, which combines the TPSS meta-GGA density functional ${ }^{\left[{ }^{[13]}\right.}$ with the BJ-damped DFT-D3 dispersion correction ${ }^{[\mathrm{S} 15]}$ and the def2-TZVP basis set, ${ }^{\left[{ }^{[16, ~ S 17]}\right.}$ using the Conductor- 
like Screening Model (COSMO) continuum solvation model ${ }^{[\mathrm{S} 18]}$ for toluene solvent (dielectric constant $\varepsilon=2.38$ and solvent diameter $\mathrm{R}_{\text {solv }}=3.48 \AA$ ). The density-fitting RI-J approach ${ }^{\left[\mathrm{S} 16, \mathrm{~S}_{19}, \mathrm{~S}^{20}\right]}$ is used to accelerate the geometry optimization and numerical harmonic frequency calculations ${ }^{[\mathrm{S} 21]}$ in solution. The optimized structures are characterized by frequency analysis to identify the nature of located stationary points (no imaginary frequency for true minima and only one imaginary frequency for transition state) and to provide thermal corrections (at $298.15 \mathrm{~K}$ and $1 \mathrm{~atm}$ ) according to the modified ideal gas-rigid rotor-harmonic oscillator model. ${ }^{[\mathrm{S} 22]}$

The final solvation free energies in toluene are computed with the COSMO-RS solvation model ${ }^{[\mathrm{S} 23]}$ (parameter file: BP_TZVP_C30_1601.ctd) using the COSMOtherm program package ${ }^{[S 24]}$ on the above TPSS-D3 optimized structures, and corrected by $+1.89 \mathrm{kcal} \cdot \mathrm{mol}^{-1}$ to account for higher reference solute concentration of $1 \mathrm{~mol} \cdot \mathrm{L}^{-1}$ usually used in solution. To check the effects of the chosen DFT functional on the reaction energies and barriers, single-point calculations at the TPSS-D ${ }^{2}$ and hybrid PW6B95-D3 ${ }^{[\mathrm{S} 25]}$ levels are performed using a larger def2-QZVP basis set. ${ }^{[\mathrm{S} 17 \text {, }}$ s26] The final reaction Gibbs free energies $(\Delta G)$ are determined from the electronic single-point energies plus TPSS-D3 thermal corrections and COSMO-RS solvation free energies. The computed reaction free energies from both DFT functionals are in good mutual agreement of $0.6 \pm 1.4 \mathrm{kcal} / \mathrm{mol}$ for reaction energies but the barriers at PW6B95-D3 level are usually $3.5 \pm 2.4 \mathrm{kcal} / \mathrm{mol}$ higher (average \pm standard deviations, see Table S1 below). In our discussion, the higher-level PW6B95-D3 Gibbs free energies (in $\mathrm{kcal} / \mathrm{mol}$, at $298.15 \mathrm{~K}$ and $1 \mathrm{~mol} / \mathrm{L}$ reference concentration) will be used in our discussion unless specified otherwise. 


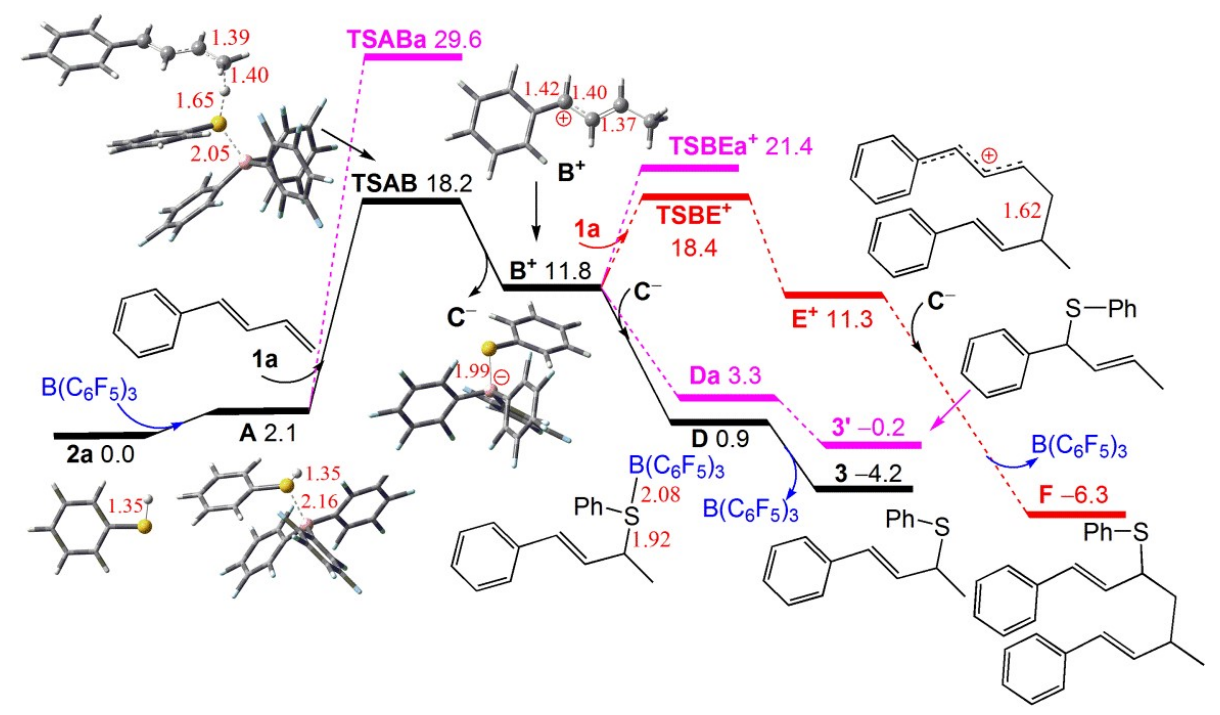

(A)

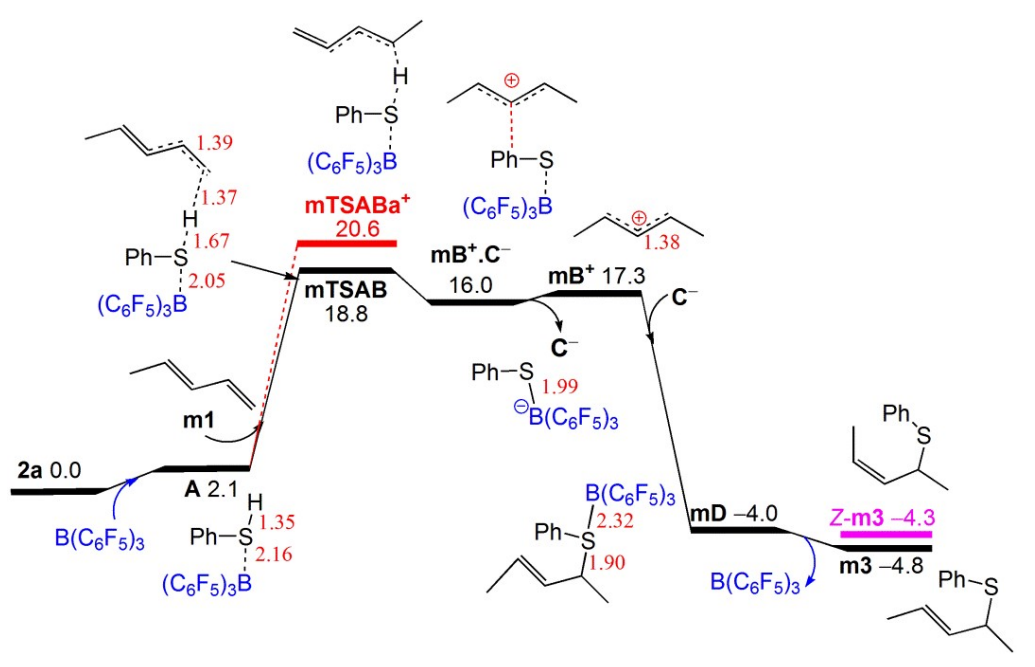

(B)
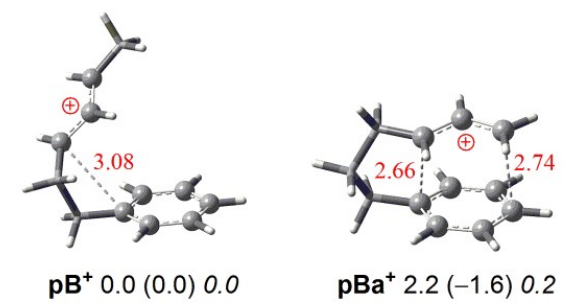

Computed $\mathrm{G}_{\mathrm{P}}\left(\mathrm{E}_{\mathrm{P}}\right) G_{T}$ values in $\mathrm{kcal} / \mathrm{mol}$

$\mathrm{G}$ : free energy $\quad \mathrm{E}$ : electronic energy suffix P: PW6B95-D3 T: TPSS-D3 level $\mathbf{p B a}^{+}$: intramolecular $\mathrm{Ph}$...allyl cation interactions

(C)

Figure S1. The free energy reaction profiles in toluene (in $\mathrm{kcal} / \mathrm{mol}$, at $298 \mathrm{~K}$ and $1 \mathrm{~mol} / \mathrm{L}$ reference concentration) for the $\mathrm{B}\left(\mathrm{C}_{6} \mathrm{~F}_{5}\right)_{3}$-catalyzed hydrothiolation of $(\mathrm{A})$ 1-phenyl 1,3-diene $\mathbf{1 a}$ and (B) 1-methyl 1,3-diene $\mathrm{m} \mathbf{1}$ using thiophenol 2a, computed at the PW6B95-D3/def2-QZVP + COSMO-RS // TPSS-D3/def2-P + COSMO level of theory. (C) DFT-supported intramolecular $\mathrm{Ph}$...allyl cation interaction model $\left(\mathrm{pBa}^{+}\right)$that may enhance the obseverved site 1 protonation. 
Table S2. TPSS-D3/def2-TZVP + COSMO computed lowest imaginary frequency (ImF), zero-point energies (ZPE), gas-phase enthalpic (Hc) and Gibbs free-energy (Gc) corrections; the COSMO-RS computed solvation enthalpic (Hsol) and Gibbs free-energy (Gsol) corrections in toluene solution; TPSS-D3/def2-QZVP and PW6B95-D3/def2-QZVP single-point energies (TPSS-D3 and PW6B95-D3); the relative electronic energies $(\triangle \mathrm{ET}$ and $\triangle \mathrm{EP})$ and Gibbs free-energies $(\triangle \mathrm{GT}$ and $\triangle \mathrm{GP}$ ) at the TPSS-D3 and PW6B95-D3 levels. Each structure is labeled either by its molecular formula or a specific name in bold. Transition structures (with only one imaginary frequency) are indicated by the "TS" prefix. See Figure S1 for structural labellings.

\begin{tabular}{|c|c|c|c|c|c|c|c|c|c|c|c|c|}
\hline Reactions & $\begin{array}{l}\mathrm{ImF} \\
\mathrm{cm}^{-1}\end{array}$ & $\begin{array}{l}\mathrm{ZPE} \\
\mathrm{kcal} / \mathrm{mol}\end{array}$ & $\begin{array}{l}\mathrm{Hc} \\
\mathrm{kca} / \mathrm{mol}\end{array}$ & $\begin{array}{l}\mathrm{Gc} \\
\mathrm{kcal} / \mathrm{mol}\end{array}$ & $\begin{array}{l}\mathrm{Hsol} \\
\mathrm{kcal} / \mathrm{mol}\end{array}$ & $\begin{array}{l}\text { Gsol } \\
\mathrm{kcal} / \mathrm{mol}\end{array}$ & $\begin{array}{l}\text { TPSS-D3 } \\
E_{h}\end{array}$ & $\begin{array}{l}\text { PW6B95-D3 } \\
E_{h}\end{array}$ & $\begin{array}{l}\Delta \mathrm{ET} \\
\mathrm{kcal} / \mathrm{mol}\end{array}$ & $\begin{array}{l}\Delta \mathrm{EP} \\
\mathrm{kcal} / \mathrm{mol}\end{array}$ & $\begin{array}{l}\Delta \mathrm{GP} \\
\mathrm{kcal} / \mathrm{mol}\end{array}$ & $\begin{array}{l}\Delta \mathrm{GT} \\
\mathrm{kcal} / \mathrm{mol}\end{array}$ \\
\hline \multicolumn{13}{|c|}{ Complexes of $B\left(C_{6} F_{5}\right)_{3}$ with thiophenol $2 a$ and 1-phenyl-1,3-diene 1a } \\
\hline $2 a+B\left(C_{6} F_{5}\right)_{3}$ & 0 & 156.04 & 179.27 & 103.46 & -27.69 & -19.27 & -2840.26808 & -2843.07386 & 0.0 & 0.0 & 0.0 & 0.0 \\
\hline A & 0 & 156.78 & 180.31 & 117.58 & -24.41 & -18.32 & -2840.28704 & -2843.09158 & -11.9 & -11.1 & 2.1 & 1.3 \\
\hline $1 a+B\left(C_{6} F_{5}\right)_{3}$ & 0 & 197.97 & 222.79 & 143.42 & -28.62 & -19.98 & -2596.93450 & -2599.60900 & 0.0 & 0.0 & 0.0 & 0.0 \\
\hline TSAa & -20 & 198.28 & 223.03 & 158.06 & -26.91 & -20.33 & -2596.93574 & -2599.60635 & -0.8 & 1.7 & 14.1 & 11.6 \\
\hline $\mathrm{Aa}$ & 0 & 198.89 & 223.85 & 158.66 & -29.09 & -22.06 & -2596.93382 & -2599.60588 & 0.4 & 2.0 & 13.2 & 11.7 \\
\hline \multicolumn{13}{|c|}{ Hydrothiolation via protonation of 1,3-diene 1a and sulfide anion transfer } \\
\hline $1 a+2 a+B\left(C_{6} F_{5}\right)_{3}$ & 0 & 259.75 & 288.97 & 186.29 & -39.38 & -26.90 & -3227.58037 & -3230.80853 & 0.0 & 0.0 & 0.0 & 0.0 \\
\hline $1 a+A$ & 0 & 260.49 & 290.02 & 200.40 & -36.10 & -25.95 & -3227.59933 & -3230.82624 & -11.9 & -11.1 & 2.1 & 1.3 \\
\hline TSAB (at site 4$)$ & -1131 & 258.14 & 288.19 & 212.38 & -34.73 & -26.58 & -3227.59606 & -3230.81554 & -9.9 & -4.4 & 18.2 & 12.8 \\
\hline $\mathrm{B}^{+}+\mathrm{C}^{-}$ & 0 & 261.26 & 290.93 & 200.89 & -101.75 & -89.60 & -3227.48393 & -3230.71014 & 60.5 & 61.8 & 11.8 & 10.5 \\
\hline D & 0 & 263.27 & 292.95 & 218.42 & -30.57 & -23.08 & -3227.63118 & -3230.85833 & -31.9 & -31.3 & 0.9 & 0.3 \\
\hline $3+\mathrm{B}\left(\mathrm{C}_{6} \mathrm{~F}_{5}\right)_{3}$ & 0 & 262.43 & 291.76 & 202.58 & -36.10 & -25.76 & -3227.61186 & -3230.83993 & -19.8 & -19.7 & -4.2 & -4.2 \\
\hline TSABa (at site 1) & -3 & 259.19 & 288.90 & 214.38 & -33.53 & -25.70 & -3227.58342 & -3230.80199 & -1.9 & 4.1 & 29.6 & 23.6 \\
\hline $\mathrm{Ba}^{+} \cdot \mathrm{C}^{-}$ & 0 & 262.13 & 292.33 & 216.85 & -30.95 & -23.86 & -3227.62028 & -3230.84152 & -25.1 & -20.7 & 9.1 & 4.8 \\
\hline $\mathrm{Da}$ & 0 & 262.66 & 292.55 & 217.46 & -30.55 & -23.11 & -3227.62569 & -3230.85296 & -28.4 & -27.9 & 3.3 & 2.8 \\
\hline $3^{\prime}+\mathrm{B}\left(\mathrm{C}_{6} \mathrm{~F}_{5}\right)_{3}$ & 0 & 262.02 & 291.49 & 201.98 & -36.08 & -25.75 & -3227.60432 & -3230.83273 & -15.0 & -15.2 & -0.2 & -0.1 \\
\hline G (via unstable $\mathbf{A a})$ & 0 & 261.09 & 291.21 & 215.73 & -33.59 & -25.53 & -3227.59587 & -3230.81961 & -9.7 & -7.0 & 20.1 & 17.3 \\
\hline TS3G & -1183 & 258.56 & 287.98 & 213.86 & -31.80 & -24.11 & -3227.53489 & -3230.75842 & 28.6 & 31.5 & 58.0 & 55.1 \\
\hline $3+\mathrm{B}\left(\mathrm{C}_{6} \mathrm{~F}_{5}\right)_{3}$ & 0 & 262.43 & 291.76 & 202.58 & -36.10 & -25.76 & -3227.61186 & -3230.83993 & -19.8 & -19.7 & -4.2 & -4.2 \\
\hline \multicolumn{13}{|c|}{ Competing 1,3-diene addition to intermediate $\boldsymbol{B}^{+}$and sulfide anion transfer from $\mathbf{C}^{-}$} \\
\hline $1 a+B^{+}$ & 0 & 214.93 & 227.25 & 172.80 & -62.37 & -52.69 & -774.98846 & -775.82730 & 0.0 & 0.0 & 11.8 & 0.0 \\
\hline TSBE$^{+}$(at site 3 ) & -193 & 215.47 & 227.66 & 187.96 & -51.48 & -45.01 & -775.01934 & -775.85015 & -19.4 & -14.3 & 18.4 & 1.6 \\
\hline
\end{tabular}




\begin{tabular}{|c|c|c|c|c|c|c|c|c|c|c|c|c|}
\hline $\mathrm{E}^{+}$ & 0 & 217.88 & 229.81 & 190.57 & -52.51 & -45.71 & -775.02683 & -775.86451 & -24.1 & -23.4 & 11.3 & -1.2 \\
\hline TSBEa $^{+}$(at site 1$)$ & -289 & 215.40 & 227.73 & 187.39 & -52.14 & -44.91 & -775.01314 & -775.84465 & -15.5 & -10.9 & 21.4 & 5.0 \\
\hline $\mathrm{Ea}^{+}$ & 0 & 217.45 & 229.57 & 189.72 & -53.91 & -46.39 & -775.01627 & -775.85408 & -17.5 & -16.8 & 16.3 & 3.9 \\
\hline $\mathrm{E}^{+}+\mathrm{C}^{-}$ & 0 & 367.93 & 403.20 & 301.49 & -103.58 & -90.25 & -3614.83460 & -3618.48201 & 0.0 & 0.0 & 0.0 & 0.0 \\
\hline $\mathbf{F}+\mathrm{B}\left(\mathrm{C}_{6} \mathrm{~F}_{5}\right)_{3}$ & 0 & 368.13 & 403.45 & 302.04 & -43.50 & -31.47 & -3614.95295 & -3618.60463 & -74.3 & -77.0 & -17.6 & -14.9 \\
\hline \multicolumn{13}{|c|}{ Potential E-to-Z conversion via $B^{+}$rotation (high overall barrier of $11.8+18.7=30.5 \mathrm{kcal} / \mathrm{mol}$ ) } \\
\hline $\mathbf{B}^{+} \quad(E$-allyl cation $)$ & 0 & 111.22 & 117.54 & 89.97 & -50.67 & -45.06 & -387.67617 & -388.09264 & 0.0 & 0.0 & 0.0 & 0.0 \\
\hline TSBz $^{+}$ & -383 & 109.98 & 116.13 & 88.92 & -51.61 & -45.98 & -387.64216 & -388.05972 & 21.3 & 20.7 & 18.7 & 19.4 \\
\hline $\mathrm{Bz}^{+}$(Z-allyl cation) & 0 & 111.34 & 117.52 & 90.31 & -50.92 & -45.40 & -387.66675 & -388.08287 & 5.9 & 6.1 & 6.1 & 5.9 \\
\hline \multicolumn{13}{|c|}{ Hydrothiolation via protonation of 1-methyl 1,3-diene $\mathbf{m} 1$ and sulfide anion transfer } \\
\hline $\mathbf{m} 1+\mathbf{2 a}+\mathrm{B}\left(\mathrm{C}_{6} \mathrm{~F}_{5}\right)_{3}$ & 0 & 226.24 & 253.80 & 155.78 & -33.95 & -22.70 & -3035.71112 & -3038.72469 & 0.0 & 0.0 & 0.0 & 0.0 \\
\hline mTSAB & -1000 & 225.01 & 253.21 & 181.19 & -30.69 & -23.54 & -3035.72288 & -3038.72795 & -7.4 & -2.1 & 18.8 & 13.4 \\
\hline mTSABa & -952 & 225.63 & 253.47 & 182.63 & -28.56 & -21.71 & -3035.72410 & -3038.73019 & -8.2 & -3.5 & 20.6 & 15.9 \\
\hline $\mathrm{mB}^{+} \cdot \mathrm{C}^{-}$ & 0 & 228.97 & 256.88 & 186.08 & -32.32 & -25.07 & -3035.73348 & -3038.73763 & -14.0 & -8.1 & 16.0 & 10.1 \\
\hline $\mathrm{mB}^{+}+\mathrm{C}^{-}$ & 0 & 227.40 & 255.30 & 170.12 & -104.52 & -93.01 & -3035.59175 & -3038.60491 & 74.9 & 75.2 & 17.3 & 17.1 \\
\hline $\mathrm{mD}$ & 0 & 229.99 & 257.95 & 186.85 & -26.42 & -19.89 & -3035.76545 & -3038.77898 & -34.1 & -34.1 & -4.0 & -4.0 \\
\hline $\mathrm{m} 3+\mathrm{B}\left(\mathrm{C}_{6} \mathrm{~F}_{5}\right)_{3}$ & 0 & 229.34 & 256.83 & 171.66 & -31.12 & -21.85 & -3035.74252 & -3038.75599 & -19.7 & -19.6 & -4.8 & -4.9 \\
\hline \multicolumn{13}{|c|}{ Protonated 1-phenethyl 1,3-diene p1: 1-phenethyl-directed protonation at site 1 \& unfavorable at site 3} \\
\hline $\mathrm{pB}^{+}$(at site 4 : missing) & 0 & 146.00 & 154.00 & 122.77 & -53.13 & -47.05 & -466.33783 & -466.83718 & 0.0 & 0.0 & 0.0 & 0.0 \\
\hline $\mathrm{pBa}^{+}$(at site 1$)$ & 0 & 146.84 & 154.24 & 124.76 & -50.35 & -45.24 & -466.34360 & -466.83975 & -3.6 & -1.6 & 2.2 & 0.2 \\
\hline $\mathrm{pBh}^{+}$(at site 3) & 0 & 147.74 & 154.72 & 126.04 & -52.17 & -46.85 & -466.30347 & -466.80171 & 21.6 & 22.3 & 25.7 & 25.0 \\
\hline
\end{tabular}


Table S3. The TPSS-D3/def2-TZVP + COSMO optimized atomic Cartesian coordinates (in $\AA$ ) in toluene. Each structure is labeled by the specific name (See also Figure S1 and Table S2), followed by the number of atoms, the total energy, and the detailed atomic coordinates.

\begin{tabular}{lrrr}
$1 \mathrm{a}$ & $\mathrm{PhCH}=\mathrm{CHCH}=\mathrm{CH}_{2}$ & \\
20 & \multicolumn{3}{l}{} \\
Energy $=-387.2948935894$ \\
$\mathrm{C}$ & -7.8843669 & -0.4276773 & 0.0617659 \\
$\mathrm{C}$ & -6.5420996 & -0.5383881 & 0.0613318 \\
$\mathrm{H}$ & -8.3747102 & 0.5408702 & 0.0616884 \\
$\mathrm{H}$ & -8.5247133 & -1.3072277 & 0.0620084 \\
$\mathrm{H}$ & -5.9315868 & 0.3654308 & 0.0610112 \\
$\mathrm{C}$ & -5.8312878 & -1.7970003 & 0.0612815 \\
$\mathrm{C}$ & -4.4802946 & -1.8885303 & 0.0609842 \\
$\mathrm{H}$ & -6.4420800 & -2.6995718 & 0.0614978 \\
$\mathrm{H}$ & -3.9065735 & -0.9606912 & 0.0607235 \\
$\mathrm{C}$ & -3.6821344 & -3.1106027 & 0.0609795 \\
$\mathrm{C}$ & -2.2775327 & -3.0028957 & 0.0609938 \\
$\mathrm{C}$ & -4.2461457 & -4.4031878 & 0.0610740 \\
$\mathrm{C}$ & -1.4659870 & -4.1359021 & 0.0611142 \\
$\mathrm{H}$ & -1.8263798 & -2.0133134 & 0.0608928 \\
$\mathrm{C}$ & -3.4357703 & -5.5333747 & 0.0612260 \\
$\mathrm{H}$ & -5.3256650 & -4.5239722 & 0.0610329 \\
$\mathrm{C}$ & -2.0415543 & -5.4078451 & 0.0612756 \\
$\mathrm{H}$ & -0.3849701 & -4.0260352 & 0.0611220 \\
$\mathrm{H}$ & -3.8900594 & -6.5203427 & 0.0613070 \\
$\mathrm{H}$ & -1.4133125 & -6.2939245 & 0.0614190
\end{tabular}

$2 \mathrm{a} \quad \mathrm{PhSH}$

13

Energy $=-630.6254585196$

$\begin{array}{llll}\mathrm{S} & -4.6158371 & -1.6524170 & 0.0602695 \\ \mathrm{H} & -3.6283100 & -0.7388677 & 0.0611306 \\ \mathrm{C} & -3.5759938 & -3.0917279 & 0.0606331 \\ \mathrm{C} & -2.1770811 & -3.0078258 & 0.0604751 \\ \mathrm{C} & -4.1977888 & -4.3487146 & 0.0608990 \\ \mathrm{C} & -1.4124140 & -4.1746561 & 0.0606909 \\ \mathrm{H} & -1.6877788 & -2.0379614 & 0.0602183 \\ \mathrm{C} & -3.4223702 & -5.5074179 & 0.0610726 \\ \mathrm{H} & -5.2821238 & -4.4198058 & 0.0610048 \\ \mathrm{C} & -2.0278136 & -5.4282431 & 0.0608984 \\ \mathrm{H} & -0.3285876 & -4.0981524 & 0.0604787 \\ \mathrm{H} & -3.9147166 & -6.4758292 & 0.0612943 \\ \mathrm{H} & -1.4281371 & -6.3333898 & 0.0611083\end{array}$

3' $\mathrm{PhCH}=\mathrm{CHCH}_{2} \mathrm{CH}_{2} \mathrm{SPh}$ (3, 4-hydrothiolation)

33

Energy $=-1017.941235813$

$\begin{array}{llrr}\text { S } & 0.6665961 & 0.3959312 & -0.7903999 \\ \text { C } & 0.9787413 & -1.1014329 & -1.6972344 \\ \text { C } & 1.9969407 & -1.9741628 & -1.2866879\end{array}$




$\begin{array}{lrrr}\mathrm{C} & 0.2049023 & -1.4174642 & -2.8224979 \\ \mathrm{C} & 2.2439378 & -3.1453504 & -2.0031016 \\ \mathrm{H} & 2.5865421 & -1.7283145 & -0.4088515 \\ \mathrm{C} & 0.4527860 & -2.5925161 & -3.5327688 \\ \mathrm{H} & -0.5798435 & -0.7373970 & -3.1375045 \\ \mathrm{C} & 1.4730188 & -3.4552003 & -3.1268269 \\ \mathrm{H} & 3.0357413 & -3.8169496 & -1.6831918 \\ \mathrm{H} & -0.1493184 & -2.8318492 & -4.4048063 \\ \mathrm{H} & 1.6654039 & -4.3696711 & -3.6809945 \\ \mathrm{C} & 1.8495602 & 1.6064808 & -1.6516063 \\ \mathrm{H} & 1.6308622 & 2.5239725 & -1.0968394 \\ \mathrm{C} & 3.2683260 & 1.2019304 & -1.4245231 \\ \mathrm{C} & 4.1145256 & 1.8802482 & -0.6419835 \\ \mathrm{H} & 3.6101460 & 0.2961750 & -1.9236980 \\ \mathrm{H} & 3.7597704 & 2.7841617 & -0.1429918 \\ \mathrm{C} & 1.4362196 & 1.7769380 & -3.0906329 \\ \mathrm{C} & 0.4986305 & 2.7649534 & -3.4240510 \\ \mathrm{C} & 1.9389034 & 0.9563918 & -4.1088737 \\ \mathrm{C} & 0.0715026 & 2.9295639 & -4.7399803 \\ \mathrm{H} & 0.1016932 & 3.4067072 & -2.6406906 \\ \mathrm{C} & 1.5083016 & 1.1160695 & -5.4271470 \\ \mathrm{H} & 2.6637977 & 0.1832504 & -3.8763041 \\ \mathrm{C} & 0.5742243 & 2.1014067 & -5.7480688 \\ \mathrm{H} & -0.6518948 & 3.7039241 & -4.9804484 \\ \mathrm{H} & 1.9039557 & 0.4668689 & -6.2034627 \\ \mathrm{H} & 0.2414068 & 2.2263213 & -6.7746685 \\ \mathrm{C} & 5.5373327 & 1.4871517 & -0.3824321 \\ \mathrm{H} & 5.8069232 & 0.5802228 & -0.9327473 \\ \mathrm{H} & 6.2272094 & 2.2896169 & -0.6740803 \\ \mathrm{H} & 5.7019270 & 1.3057742 & 0.6881014\end{array}$

$3 \mathrm{PhCH}=\mathrm{CHCH}(\mathrm{SPh}) \mathrm{CH}_{3}$ (4,3-hydrothiolation) 33

Energy $=-1017.948696586$

$\begin{array}{lrrr}\mathrm{C} & -0.7268294 & 2.7190632 & -2.6793240 \\ \mathrm{C} & -0.8121445 & 1.7609962 & -1.4925774 \\ \mathrm{H} & -0.3904375 & 2.1958688 & -3.5807522 \\ \mathrm{H} & -1.6983431 & 3.1795419 & -2.8819753 \\ \mathrm{H} & -1.2005412 & 2.2701572 & -0.6065721 \\ \mathrm{C} & 0.4585178 & 1.0536630 & -1.1901255 \\ \mathrm{C} & 0.8518411 & 0.7424902 & 0.0585407 \\ \mathrm{H} & 1.0280543 & 0.7153425 & -2.0552526 \\ \mathrm{H} & 0.2206297 & 1.0585289 & 0.8895132 \\ \mathrm{C} & 2.0362951 & -0.0305086 & 0.4355430 \\ \mathrm{C} & 2.1630373 & -0.4775522 & 1.7633062 \\ \mathrm{C} & 3.0601022 & -0.3588516 & -0.4739450 \\ \mathrm{C} & 3.2640793 & -1.2309687 & 2.1687330 \\ \mathrm{H} & 1.3795466 & -0.2355357 & 2.4777240 \\ \mathrm{C} & 4.1605362 & -1.1082298 & -0.0696248 \\ \mathrm{H} & 2.9994496 & -0.0148715 & -1.5026088 \\ \mathrm{C} & 4.2694947 & -1.5498939 & 1.2539743 \\ \mathrm{H} & 3.3382744 & -1.5673382 & 3.1994171\end{array}$




$\begin{array}{lrrr}\mathrm{H} & 4.9414504 & -1.3460949 & -0.7869711 \\ \mathrm{H} & 5.1322504 & -2.1309049 & 1.5668590 \\ \mathrm{~S} & -2.1131770 & 0.4591933 & -1.9170895 \\ \mathrm{H} & -0.0044458 & 3.5108403 & -2.4540816 \\ \mathrm{C} & -2.1731234 & -0.3845817 & -0.3517747 \\ \mathrm{C} & -2.9116723 & 0.1588875 & 0.7077376 \\ \mathrm{C} & -1.4398557 & -1.5615478 & -0.1559055 \\ \mathrm{C} & -2.9139356 & -0.4703333 & 1.9521794 \\ \mathrm{H} & -3.4746417 & 1.0749273 & 0.5534949 \\ \mathrm{C} & -1.4522024 & -2.1926117 & 1.0888448 \\ \mathrm{H} & -0.8539880 & -1.9674847 & -0.9744487 \\ \mathrm{C} & -2.1853660 & -1.6475075 & 2.1442324 \\ \mathrm{H} & -3.4838702 & -0.0414683 & 2.7715311 \\ \mathrm{H} & -0.8762244 & -3.1015564 & 1.2358915 \\ \mathrm{H} & -2.1886720 & -2.1363093 & 3.1141678\end{array}$

A. 1a B $\left(\mathrm{C}_{6} \mathrm{~F}_{5}\right)_{3}$. $\mathrm{PhSH}$. $\mathrm{PhCH}=\mathrm{CHCH}=\mathrm{CH}_{2}$ complex

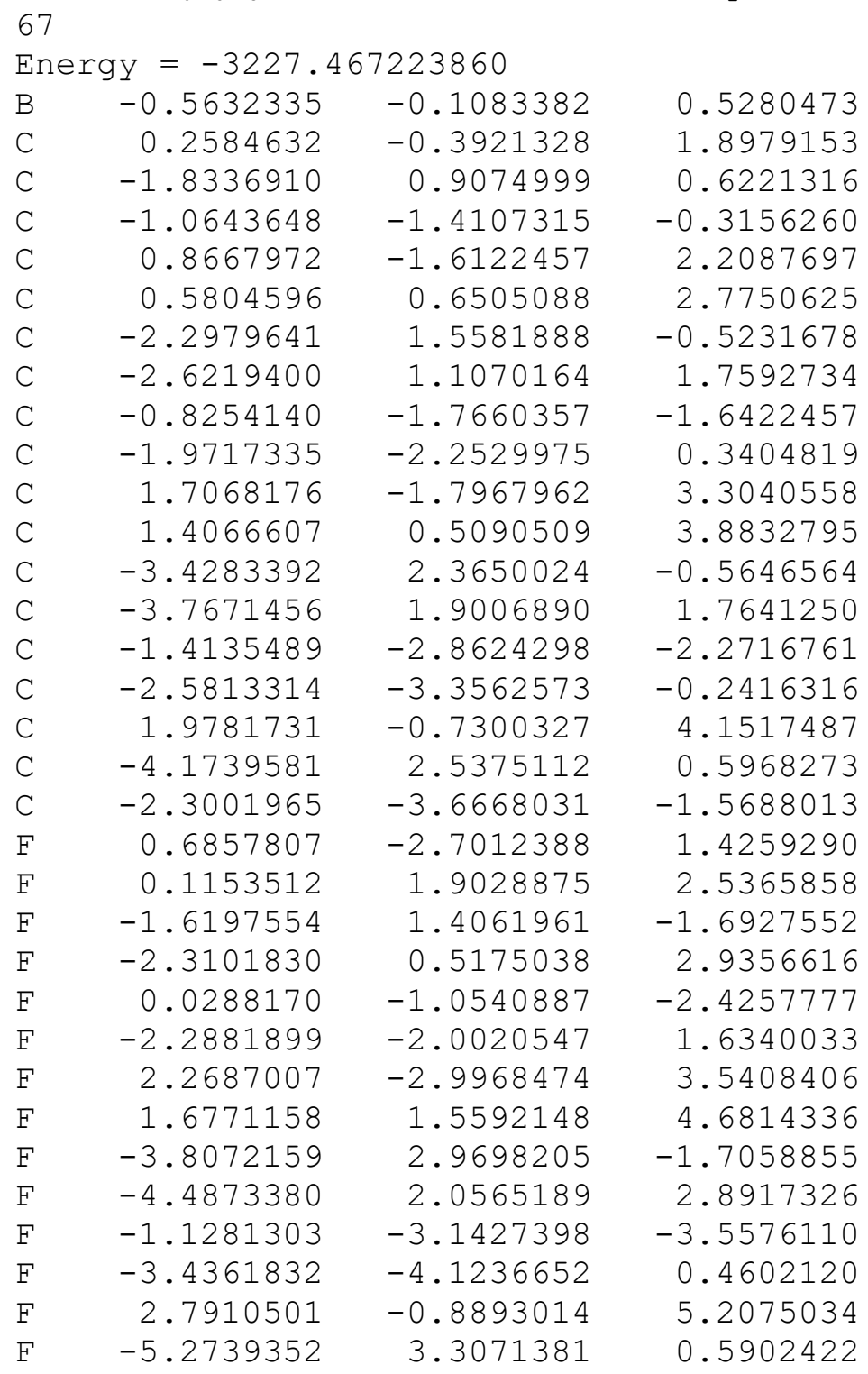




$\begin{array}{lrrr}\mathrm{F} & -2.8779383 & -4.7253123 & -2.1586423 \\ \mathrm{~S} & 0.9211976 & 1.0164926 & -0.3901055 \\ \mathrm{C} & 2.4405256 & 0.1326554 & -0.0772020 \\ \mathrm{C} & 3.3407047 & 0.7540430 & 0.7893324 \\ \mathrm{C} & 2.6928704 & -1.1397504 & -0.5952358 \\ \mathrm{C} & 4.5069528 & 0.0831966 & 1.1544300 \\ \mathrm{H} & 3.1326010 & 1.7487144 & 1.1688818 \\ \mathrm{C} & 3.8667605 & -1.7937047 & -0.2312067 \\ \mathrm{H} & 1.9879296 & -1.6135671 & -1.2663305 \\ \mathrm{C} & 4.7690409 & -1.1887059 & 0.6469676 \\ \mathrm{H} & 5.2108573 & 0.5614612 & 1.8280950 \\ \mathrm{H} & 4.0728909 & -2.7817414 & -0.6307955 \\ \mathrm{H} & 5.6787274 & -1.7088321 & 0.9305321 \\ \mathrm{C} & -1.0891219 & 0.5025224 & -5.4467456 \\ \mathrm{C} & -0.3939714 & 1.4598332 & -4.8063575 \\ \mathrm{H} & -0.6623246 & -0.4821064 & -5.6231648 \\ \mathrm{H} & -2.1007416 & 0.6747068 & -5.8004640 \\ \mathrm{H} & -0.8537924 & 2.4328190 & -4.6318472 \\ \mathrm{C} & 0.9472493 & 1.2787534 & -4.2991455 \\ \mathrm{C} & 1.6168640 & 2.2339113 & -3.6079161 \\ \mathrm{H} & 1.4106649 & 0.3103643 & -4.4853828 \\ \mathrm{H} & 1.1102564 & 3.1848340 & -3.4375881 \\ \mathrm{C} & 2.9481637 & 2.1279798 & -3.0188195 \\ \mathrm{C} & 3.4240513 & 3.1916351 & -2.2274536 \\ \mathrm{C} & 3.7732703 & 0.9960956 & -3.1740617 \\ \mathrm{C} & 4.6719690 & 3.1285233 & -1.6116531 \\ \mathrm{H} & 2.7979148 & 4.0708713 & -2.0962994 \\ \mathrm{C} & 5.0174111 & 0.9337917 & -2.5579581 \\ \mathrm{H} & 3.4346432 & 0.1573614 & -3.7753412 \\ \mathrm{C} & 5.4733395 & 1.9973942 & -1.7725989 \\ \mathrm{H} & 5.0170786 & 3.9593384 & -1.0026447 \\ \mathrm{H} & 5.6340389 & 0.0485797 & -2.6815754 \\ \mathrm{H} & 6.4425018 & 1.9398471 & -1.2862752 \\ \mathrm{H} & 0.8008417 & 0.8293916 & -1.7219650\end{array}$

Aa $\mathrm{B}\left(\mathrm{C}_{6} \mathrm{~F}_{5}\right)_{3} \cdot \mathrm{CH}_{2}=\mathrm{CHCH}=\mathrm{CHPh}$

54

Energy $=-2596.815955899$

$\begin{array}{lrr}\text { B } & 0.8295841 & 0.0756573 \\ \text { C } & 1.2637525 & 1.6131657 \\ \text { C } & -0.3132764 & -0.5935601 \\ \text { C } & 2.0871685 & -0.9854494 \\ \text { C } & 2.5729334 & 2.0982043 \\ \text { C } & 0.3259917 & 2.6054162 \\ C & -0.9675627 & -1.7710307 \\ \text { C } & -0.6814785 & -0.1421335 \\ C & 2.6238336 & -1.7334411 \\ \text { C } & 2.6985299 & -1.2323084 \\ C & 2.9318796 & 3.4261777 \\ C & 0.6339166 & 3.9402604 \\ C & -1.9578601 & -2.4033868 \\ C & -1.6604346 & -0.7467656\end{array}$

-0.5090584
-0.1754663
0.4641835
-0.5098591
-0.2801303
0.1344456
0.0833963
1.7369047
-1.5588600
0.7259187
-0.0555132
0.3644078
0.8278303
2.5239406 


$\begin{array}{lrrr} & & & \\ \mathrm{C} & 3.6572020 & -2.6572931 & -1.4031733 \\ \mathrm{C} & 3.7345456 & -2.1351693 & 0.9284950 \\ \mathrm{C} & 1.9570784 & 4.3584026 & 0.2748264 \\ \mathrm{C} & -2.3177397 & -1.8808691 & 2.0638390 \\ \mathrm{C} & 4.2199292 & -2.8643007 & -0.1514877 \\ \mathrm{~F} & 3.5971462 & 1.2813552 & -0.6319030 \\ \mathrm{~F} & -0.9946721 & 2.2893779 & 0.2095058 \\ \mathrm{~F} & -0.6501944 & -2.3825406 & -1.0918182 \\ \mathrm{~F} & -0.0733204 & 0.9248170 & 2.3054091 \\ \mathrm{~F} & 2.1763947 & -1.5963897 & -2.8352516 \\ \mathrm{~F} & 2.2952273 & -0.5364767 & 1.8194784 \\ \mathrm{~F} & 4.2172782 & 3.8166129 & -0.1680565 \\ \mathrm{~F} & -0.3321167 & 4.8305291 & 0.6666095 \\ \mathrm{~F} & -2.5651265 & -3.5154386 & 0.3636742 \\ \mathrm{~F} & -1.9691942 & -0.2451642 & 3.7369336 \\ \mathrm{~F} & 4.1183398 & -3.3507101 & -2.4647147 \\ \mathrm{~F} & 4.2754852 & -2.3054986 & 2.1520057 \\ \mathrm{~F} & 2.2831998 & 5.6437326 & 0.4905126 \\ \mathrm{~F} & -3.2683494 & -2.4728719 & 2.8066696 \\ \mathrm{~F} & 5.2197707 & -3.7481821 & 0.0128443 \\ \mathrm{C} & 0.1861355 & 0.1934391 & -2.2087264 \\ \mathrm{C} & -1.0439657 & 0.8897285 & -2.1790686 \\ \mathrm{H} & 0.9976671 & 0.7239897 & -2.7048267 \\ \mathrm{H} & 0.0916739 & -0.8448045 & -2.5103374 \\ \mathrm{H} & -1.0351579 & 1.9775457 & -2.2106683 \\ \mathrm{C} & -2.2913956 & 0.2747883 & -2.0089895 \\ \mathrm{C} & -3.4114489 & 1.0260286 & -1.7404357 \\ \mathrm{H} & -2.3425569 & -0.8090156 & -2.0385168 \\ \mathrm{H} & -3.2986505 & 2.1099819 & -1.7484198 \\ \mathrm{C} & -4.7159325 & 0.5325274 & -1.4012111 \\ \mathrm{C} & -5.7402117 & 1.4699559 & -1.1361144 \\ \mathrm{C} & -5.0069029 & -0.8479376 & -1.2902011 \\ \mathrm{C} & -7.0121324 & 1.0451472 & -0.7754291 \\ \mathrm{H} & -5.5177840 & 2.5302801 & -1.2181565 \\ \mathrm{C} & -6.2774077 & -1.2661621 & -0.9289139 \\ \mathrm{H} & -4.2350235 & -1.5851877 & -1.4850350 \\ \mathrm{C} & -7.2820439 & -0.3226459 & -0.6713521 \\ \mathrm{H} & -7.7921244 & 1.7720302 & -0.5728028 \\ \mathrm{H} & -6.4960681 & -2.3259192 & -0.8442024 \\ \mathrm{H} & -8.2750874 & -0.6577626 & -0.3875289\end{array}$

A $\mathrm{B}\left(\mathrm{C}_{6} \mathrm{~F}_{5}\right)_{3}$. PhSH complex 47

Energy $=-2840.161921022$

$\begin{array}{lrrr}\text { B } & -0.2642178 & 0.0216776 & 0.1455389 \\ \text { C } & 1.1383117 & -0.1539895 & -0.6191080 \\ \text { C } & -1.2662930 & -1.2549143 & 0.1945664 \\ \text { C } & -1.1096348 & 1.3715294 & -0.1742246 \\ \text { C } & 1.7202969 & 0.8131448 & -1.4440810 \\ \text { C } & 1.9509410 & -1.2682919 & -0.3769328 \\ \text { C } & -2.3038588 & -1.3155419 & 1.1287401 \\ \text { C } & -1.2611672 & -2.3026027 & -0.7313774\end{array}$




\begin{tabular}{|c|c|c|c|}
\hline C & -1.0942092 & 2.5785881 & 0.5229944 \\
\hline & -1.9699144 & 1.3601464 & -1.2783347 \\
\hline t & 3.0004052 & 0.6939120 & -1.9820686 \\
\hline & 3.2339946 & -1.4233160 & -0.8800981 \\
\hline & -3.2464137 & -2.3353600 & 1.1870072 \\
\hline & -2.1859499 & -3.3429818 & -0.7143579 \\
\hline C & -1.8846608 & 3.6764360 & 0.1944776 \\
\hline$C$ & -2.7749769 & 2.4312798 & -1.6485344 \\
\hline$C$ & 3.7672895 & -0.4276582 & -1.6938143 \\
\hline$C$ & -3.1843576 & -3.3646490 & 0.2537445 \\
\hline$\gamma$ & -2.7382756 & 3.6027428 & -0.8994824 \\
\hline$\vec{F}$ & 1.0645543 & 1.9563384 & -1.7530092 \\
\hline$F$ & 1.4980428 & -2.2614855 & 0.4299938 \\
\hline$F$ & -2.4246675 & -0.3258650 & 2.0551941 \\
\hline$F$ & -0.3600840 & -2.3313466 & -1.7388288 \\
\hline$F$ & -0.2567810 & 2.7586852 & 1.5810632 \\
\hline F & -2.0217768 & 0.2677893 & -2.0799901 \\
\hline $\mathrm{F}$ & 3.5055029 & 1.6644948 & -2.7662645 \\
\hline$F$ & 3.9679483 & -2.5102187 & -0.5814558 \\
\hline$F$ & -4.2098908 & -2.3345861 & 2.1260654 \\
\hline$t^{\prime}$ & -2.1295047 & -4.3241893 & -1.6340822 \\
\hline$F$ & -1.8223302 & 4.8077392 & 0.9210833 \\
\hline$F$ & -3.5786338 & 2.3490593 & -2.7248956 \\
\hline F & 5.0072771 & -0.5503364 & -2.1898740 \\
\hline$F$ & -4.0810312 & -4.3618867 & 0.2824256 \\
\hline F & -3.5053980 & 4.6507938 & -1.2367746 \\
\hline S & 0.3601141 & 0.1457902 & 2.2103940 \\
\hline $\mathrm{H}$ & 0.3505124 & -1.1821963 & 2.4316094 \\
\hline C & 2.1386793 & 0.3127119 & 2.2010281 \\
\hline C & 2.9351697 & -0.7218046 & 2.6991824 \\
\hline C & 2.7066491 & 1.4818661 & 1.6880404 \\
\hline C & 4.3220160 & -0.5850781 & 2.6663566 \\
\hline $\mathrm{H}$ & 2.4833714 & -1.6266489 & 3.0924633 \\
\hline $\mathrm{C}$ & 4.0951345 & 1.5996886 & 1.6562023 \\
\hline $\mathrm{H}$ & 2.0821841 & 2.2829813 & 1.3101882 \\
\hline C & 4.9034689 & 0.5691928 & 2.1396895 \\
\hline $\mathrm{H}$ & 4.9446546 & -1.3902073 & 3.0434716 \\
\hline 1 & 4.5420566 & 2.5016502 & 1.2501647 \\
\hline $\mathrm{H}$ & 5.9840321 & 0.6673488 & 2.1095250 \\
\hline
\end{tabular}

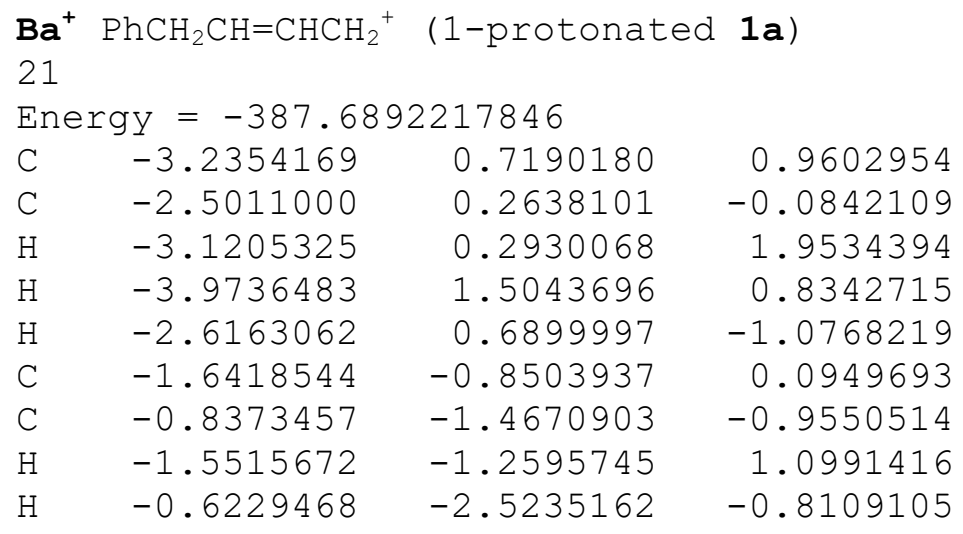




$\begin{array}{lrrr}\mathrm{C} & 0.3312982 & -0.5754943 & -0.4802902 \\ \mathrm{C} & 1.1553427 & -1.0041990 & 0.5801615 \\ \mathrm{C} & 0.5631211 & 0.6689025 & -1.1015790 \\ \mathrm{C} & 2.2409283 & -0.2271464 & 0.9636417 \\ \mathrm{H} & 0.9526974 & -1.9509422 & 1.0720425 \\ \mathrm{C} & 1.6536573 & 1.4336317 & -0.7153119 \\ \mathrm{H} & -0.0922408 & 1.0072702 & -1.8981153 \\ \mathrm{C} & 2.4897144 & 0.9888067 & 0.3178283 \\ \mathrm{H} & 2.8924388 & -0.5638697 & 1.7631498 \\ \mathrm{H} & 1.8559982 & 2.3769543 & -1.2117425 \\ \mathrm{H} & 3.3347671 & 1.5974676 & 0.6241923 \\ \mathrm{H} & -1.1419157 & -1.2304290 & -1.9723159\end{array}$

$\mathbf{B}^{+} \quad \mathrm{E}-\mathrm{PhCH}=\mathrm{CHCHCH}_{3}{ }^{+} \quad$ (4-protonated 1a)

21

Energy $=-387.7195204942$

$\begin{array}{lrrr}\mathrm{C} & -4.4324162 & 0.3244797 & 0.0074940 \\ \mathrm{C} & -3.1658724 & -0.4197076 & 0.0817744 \\ \mathrm{H} & -5.0473286 & -0.0844466 & -0.8075771 \\ \mathrm{H} & -4.2899264 & 1.3971636 & -0.1312331 \\ \mathrm{H} & -3.2305404 & -1.5011482 & 0.2033221 \\ \mathrm{C} & -1.9159178 & 0.1439271 & 0.0116548 \\ \mathrm{C} & -0.7843921 & -0.6776666 & 0.0938199 \\ \mathrm{H} & -1.8178416 & 1.2190641 & -0.1076920 \\ \mathrm{H} & -0.9649849 & -1.7457966 & 0.2096703 \\ \mathrm{C} & 0.5705534 & -0.2727407 & 0.0397252 \\ \mathrm{C} & 1.5717373 & -1.2784708 & 0.1304008 \\ \mathrm{C} & 0.9617747 & 1.0875597 & -0.1009307 \\ \mathrm{C} & 2.9119996 & -0.9378108 & 0.0800780 \\ \mathrm{H} & 1.2663814 & -2.3151218 & 0.2377110 \\ \mathrm{C} & 2.3026709 & 1.4166455 & -0.1458065 \\ \mathrm{H} & 0.2110717 & 1.8669738 & -0.1729380 \\ \mathrm{C} & 3.2762636 & 0.4080069 & -0.0566451 \\ \mathrm{H} & 3.6765503 & -1.7043174 & 0.1463634 \\ \mathrm{H} & 2.6070743 & 2.4524129 & -0.2509125 \\ \mathrm{H} & 4.3273366 & 0.6774995 & -0.0948505 \\ \mathrm{H} & -5.0147818 & 0.1382538 & 0.9225349\end{array}$

$\mathbf{B z}^{+} Z-\mathrm{PhCH}=\mathrm{CHCHCH}_{3}{ }^{+}$

21

Energy $=-387.7103449718$

$\begin{array}{lrrr}\mathrm{C} & -3.1187825 & 2.3265034 & 0.5630154 \\ \mathrm{C} & -1.9268701 & 1.4647104 & 0.4886324 \\ \mathrm{H} & -3.3148239 & 2.6090241 & 1.6064236 \\ \mathrm{H} & -4.0040609 & 1.8688414 & 0.1193704 \\ \mathrm{H} & -1.0248973 & 1.8334835 & 0.9732168 \\ \mathrm{C} & -1.9109782 & 0.2126018 & -0.0729511 \\ \mathrm{C} & -0.7772381 & -0.6189156 & -0.1278408 \\ \mathrm{H} & -2.8549910 & -0.2197720 & -0.3974717 \\ \mathrm{H} & -0.9713294 & -1.6876480 & -0.2128268 \\ \mathrm{C} & 0.5867789 & -0.2430453 & -0.0863924 \\ \mathrm{C} & 1.5384234 & -1.2495778 & 0.2413447\end{array}$




\begin{tabular}{|c|c|c|c|}
\hline C & 1.0414905 & 1.0786481 & -0.3537044 \\
\hline C & 2.8746337 & -0.9233841 & 0.3871418 \\
\hline $\mathrm{H}$ & 1.1928302 & -2.2648959 & 0.4117074 \\
\hline $\mathrm{C}$ & 2.3816957 & 1.3892715 & -0.2187274 \\
\hline $\mathrm{H}$ & 0.3469590 & 1.8213766 & -0.7299677 \\
\hline $\mathrm{C}$ & 3.2950645 & 0.3951604 & 0.1643300 \\
\hline $\mathrm{H}$ & 3.5970893 & -1.6843528 & 0.6616741 \\
\hline $\mathrm{H}$ & 2.7319711 & 2.3925400 & -0.4367653 \\
\hline & 4.3477726 & 0.6438644 & 0.2574992 \\
\hline & -2.8980599 & 3.2736476 & 0.0461489 \\
\hline & \multicolumn{3}{|c|}{$\left(\mathrm{C}_{6} \mathrm{~F}_{5}\right){ }_{3} \mathrm{BSPh}^{-}$anion } \\
\hline \multicolumn{4}{|c|}{ Energy $=-2839.719473233$} \\
\hline B & -0.1523078 & -0.0817498 & 0.3527194 \\
\hline C & 1.1526646 & -0.3325463 & -0.5983205 \\
\hline C & -1.3003594 & -1.2658255 & 0.2983905 \\
\hline C & -0.9679762 & 1.3130528 & 0.0156916 \\
\hline $\mathrm{C}$ & 1.7380402 & 0.6262410 & -1.4275627 \\
\hline $\mathrm{C}$ & 1.8964915 & -1.5157983 & -0.4981771 \\
\hline $\mathrm{C}$ & -2.3380196 & -1.2693081 & 1.2367585 \\
\hline C & -1.4059023 & -2.2735472 & -0.6 \\
\hline C & -1.1025528 & 2.45121 & 0.8 \\
\hline C & -1.6788727 & 1.3813 & -1 \\
\hline $\mathrm{C}$ & 2.9456733 & 0.4466817 & -2.1000965 \\
\hline $\mathrm{C}$ & 3.1054334 & -1.7365668 & -1.1442769 \\
\hline $\mathrm{C}$ & -3.3682386 & -2.2032796 & 1.2651387 \\
\hline $\mathrm{C}$ & -2.4164368 & -3.2329011 & -0.6719888 \\
\hline$C$ & -1.8868559 & 3.5512691 & 0.4604809 \\
\hline $\mathrm{C}$ & -2.4690095 & 2.4534506 & -1.5815556 \\
\hline C & 3.6413485 & -0.7437272 & -1.9577087 \\
\hline $\mathrm{C}$ & -3.4055258 & -3.20475 & 23206 \\
\hline C & -2.5786235 & 3.55 & -0.7423438 \\
\hline $\mathrm{F}$ & 1.1695336 & 1.8494581 & -1.6052463 \\
\hline $\mathrm{F}$ & 1.4542894 & -2.5357190 & 0.2759839 \\
\hline $\mathrm{F}$ & -2.3927915 & -0.3019310 & 2.1901475 \\
\hline $\mathrm{F}$ & -0.5258806 & -2.3596938 & -1.6913437 \\
\hline $\mathrm{F}$ & -0.4543708 & 2.5761310 & 1.9956877 \\
\hline $\mathrm{F}$ & -1.5877160 & 0.3560782 & -2.0767954 \\
\hline $\mathrm{F}$ & 3.4553723 & 1.4323435 & -2.8733669 \\
\hline $\mathrm{F}$ & 3.7678543 & -2.90 & -0.9944832 \\
\hline $\mathrm{F}$ & -4.3328882 & -2.1455 & 2.2098695 \\
\hline $\mathrm{F}$ & -2.4521669 & -4.1850584 & -1.6311264 \\
\hline $\mathrm{F}$ & -1.9761804 & 4.6204899 & 1.2837195 \\
\hline $\mathrm{F}$ & -3.1180235 & 2.4449075 & -2.7674317 \\
\hline $\mathrm{F}$ & 4.8145612 & -0.9365611 & -2.5929712 \\
\hline $\mathrm{F}$ & -4.3885200 & -4.1263718 & 0.3095737 \\
\hline $\mathrm{F}$ & -3.3365147 & 4.6138839 & -1.0951922 \\
\hline S & 0.5412117 & -0.2459026 & 2.2080286 \\
\hline $\mathrm{C}$ & 2.2220599 & 0.2991823 & 2.1611364 \\
\hline $\mathrm{C}$ & 3.1860828 & -0.4503899 & 2.8571634 \\
\hline $\mathrm{C}$ & 2.6338167 & 1.4592505 & 1.483535 \\
\hline
\end{tabular}




$\begin{array}{lrrr}\mathrm{C} & 4.5216995 & -0.0497986 & 2.8757878 \\ \mathrm{H} & 2.8806660 & -1.3578334 & 3.3713708 \\ \mathrm{C} & 3.9745732 & 1.8416392 & 1.4846993 \\ \mathrm{H} & 1.9006136 & 2.0621027 & 0.9588949 \\ \mathrm{C} & 4.9264888 & 1.0936782 & 2.1824460 \\ \mathrm{H} & 5.2512375 & -0.6452893 & 3.4191269 \\ \mathrm{H} & 4.2755693 & 2.7350533 & 0.9430463 \\ \mathrm{H} & 5.9698112 & 1.3966602 & 2.1836576\end{array}$

Da $\mathrm{CH}_{3} \mathrm{CH}=\mathrm{CHCHPhSPh} . \mathrm{B}\left(\mathrm{C}_{6} \mathrm{~F}_{5}\right)_{3}$ complex

67

Energy $=-3227.479507112$

\begin{tabular}{|c|c|c|c|}
\hline 3 & -0.9836267 & 0.0934695 & -0.0992046 \\
\hline & 0.0943876 & -0.3016043 & 1.0448774 \\
\hline & -1.5287048 & 1.6355749 & -0.1176686 \\
\hline & -2.3205608 & -0.8557208 & -0.1567369 \\
\hline & 0.2000810 & -1.5973976 & 1.5651032 \\
\hline & 1.0881171 & 0.5721284 & 1.5016427 \\
\hline & -2.1066362 & 2.1915411 & -1.2653650 \\
\hline & -1.6250715 & 2.4520296 & 1.0175738 \\
\hline & -2.8938880 & -1.5194273 & -1.2427912 \\
\hline & -3.0740465 & -0.9470415 & 1.0207779 \\
\hline & 1.1762361 & -1.9921035 & 2.4771057 \\
\hline & 2.0874911 & 0.2196358 & 2.4011065 \\
\hline & -2.6610440 & 3.4656191 & -1.3274941 \\
\hline & -2.1784076 & 3.7303339 & 1.0017683 \\
\hline C & -4.0956544 & -2.2241185 & -1.1738732 \\
\hline $\mathrm{C}$ & -4.2728885 & -1.6385243 & 1.1373178 \\
\hline $\mathrm{C}$ & 2.1323603 & -1.0786030 & 2.9004799 \\
\hline $\mathrm{C}$ & -2.6914566 & 4.2500714 & -0.1805619 \\
\hline & -4.7945351 & -2.2874390 & 0.0235374 \\
\hline & -0.6437430 & -2.5766256 & 1.1662574 \\
\hline$F$ & 1.1424879 & 1.8531465 & 1.0530114 \\
\hline $\mathrm{F}$ & -2.1640601 & 1.4669683 & -2.4101160 \\
\hline $\mathrm{F}$ & -1.1999975 & 2.0267295 & 2.2281822 \\
\hline F & -2.3011997 & -1.5289439 & -2.4630008 \\
\hline $\mathrm{F}$ & -2.6264604 & -0.3393347 & 2.1471029 \\
\hline F & 1.2232673 & -3.2616211 & 2.9209122 \\
\hline $\mathrm{F}$ & 3.0125378 & 1.1186674 & 2.7873449 \\
\hline $\mathrm{F}$ & -3.1754995 & 3.9370637 & -2.4785177 \\
\hline 1 & -2.2284591 & 4.4654439 & 2.1291681 \\
\hline $\mathrm{F}$ & -4.5844466 & -2.8427228 & -2.2670894 \\
\hline F & -4.9301042 & -1.6856600 & 2.3121132 \\
\hline $\mathrm{F}$ & 3.0926879 & -1.4437786 & 3.7635136 \\
\hline F & -3.2244264 & 5.4812767 & -0.2102299 \\
\hline $\mathrm{F}$ & -5.9540081 & -2.9604226 & 0.1057544 \\
\hline S & 0.0758829 & -0.1760126 & -1.8679739 \\
\hline C & 1.0349399 & -1.6687119 & -1.7030437 \\
\hline $\mathrm{C}$ & 2.0768457 & -1.8469758 & -0.7855722 \\
\hline $\mathrm{C}$ & 0.6815635 & -2.6964834 & -2.5872679 \\
\hline $\mathrm{C}$ & 2.7531150 & -3.0647621 & -0.7499555 \\
\hline $\mathrm{H}$ & 2.3692147 & -1.0434580 & -0.124572 \\
\hline
\end{tabular}




$\begin{array}{rrrr}\mathrm{C} & 1.3579745 & -3.9134878 & -2.5335702 \\ \mathrm{H} & -0.1180238 & -2.5416931 & -3.3031364 \\ \mathrm{C} & 2.3928942 & -4.0990514 & -1.6153481 \\ \mathrm{H} & 3.5631835 & -3.2042563 & -0.0402318 \\ \mathrm{H} & 1.0798794 & -4.7113107 & -3.2148426 \\ \mathrm{H} & 2.9214077 & -5.0466162 & -1.5771959 \\ \mathrm{C} & 1.3017730 & 1.2975930 & -2.0112179 \\ \mathrm{H} & 1.0443582 & 1.9348703 & -1.1668517 \\ \mathrm{C} & 2.7272029 & 0.8667660 & -1.9437360 \\ \mathrm{C} & 3.5835537 & 1.3124747 & -1.0172202 \\ \mathrm{H} & 3.0756271 & 0.2022692 & -2.7326649 \\ \mathrm{H} & 3.2206318 & 1.9887989 & -0.2446150 \\ \mathrm{C} & 0.9527136 & 1.9819272 & -3.3111946 \\ \mathrm{C} & 0.4943489 & 3.3020763 & -3.2880395 \\ \mathrm{C} & 1.1115571 & 1.3296642 & -4.5404378 \\ \mathrm{C} & 0.1975195 & 3.9660392 & -4.4791906 \\ \mathrm{H} & 0.3722631 & 3.8112545 & -2.3354455 \\ \mathrm{C} & 0.8194969 & 1.9926860 & -5.7291961 \\ \mathrm{H} & 1.4559128 & 0.2988282 & -4.5690648 \\ \mathrm{C} & 0.3613094 & 3.3135230 & -5.7015189 \\ \mathrm{H} & -0.1619058 & 4.9903969 & -4.4509945 \\ \mathrm{H} & 0.9477825 & 1.4804486 & -6.6782245 \\ \mathrm{H} & 0.1305876 & 3.8281998 & -6.6296972 \\ \mathrm{C} & 5.0321927 & 0.9448234 & -0.9577641 \\ \mathrm{H} & 5.3105737 & 0.2632574 & -1.7666094 \\ \mathrm{H} & 5.6629581 & 1.8403406 & -1.0179045 \\ \mathrm{H} & 5.2652482 & 0.4644693 & 0.0017485\end{array}$

D $\mathrm{PhCH}=\mathrm{CHCHMeSPh} . \mathrm{B}\left(\mathrm{C}_{6} \mathrm{~F}_{5}\right)_{3}$ complex

67

Energy $=-3227.484977325$

\begin{tabular}{|c|c|c|c|}
\hline B & -1.0025066 & 0.1150844 & -0.0757829 \\
\hline$\sim$ & 0.0873825 & -0.2856189 & 1.0537421 \\
\hline $\mathrm{C}$ & -1.5875572 & 1.6431313 & -0.0459570 \\
\hline C & -2.3194867 & -0.8619451 & -0.1513455 \\
\hline$C$ & 0.2214030 & -1.5880606 & 1.5498888 \\
\hline & 1.0665786 & 0.5999728 & 1.5182473 \\
\hline & -2.1934692 & 2.2167152 & -1.1698870 \\
\hline & -1.7022071 & 2.4214155 & 1.1144092 \\
\hline & -2.8803587 & -1.5191654 & -1.2477384 \\
\hline & -3.0707412 & -0.9890289 & 1.0247598 \\
\hline & 1.2161988 & -1.9800711 & 2.4430861 \\
\hline & 2.0802778 & 0.2521667 & 2.4033471 \\
\hline & -2.7962979 & 3.4694440 & -1.1881882 \\
\hline & -2.3063532 & 3.6764751 & 1.1442394 \\
\hline & -4.0655339 & -2.2524317 & -1.1893218 \\
\hline & -4.2549603 & -1.7068329 & 1.1300988 \\
\hline & 2.1588500 & -1.0555649 & 2.8731956 \\
\hline & -2.8508454 & 4.2135923 & -0.0157957 \\
\hline & -4.7627585 & -2.3501361 & 0.0065098 \\
\hline & -0.6088800 & -2.5755832 & 1.1428262 \\
\hline & 1.0859492 & 1.8891283 & 1.09212 \\
\hline
\end{tabular}




$\begin{array}{lrrr}\text { F } & -2.2319561 & 1.5294388 & -2.3415131 \\ \mathrm{~F} & -1.2443568 & 1.9795851 & 2.3067948 \\ \mathrm{~F} & -2.2920406 & -1.4918035 & -2.4698493 \\ \mathrm{~F} & -2.6362782 & -0.3898161 & 2.1606472 \\ \mathrm{~F} & 1.2934767 & -3.2566371 & 2.8623460 \\ \mathrm{~F} & 2.9832572 & 1.1645110 & 2.8079987 \\ \mathrm{~F} & -3.3359830 & 3.9565610 & -2.3213723 \\ \mathrm{~F} & -2.3723493 & 4.3729347 & 2.2949144 \\ \mathrm{~F} & -4.5404269 & -2.8644430 & -2.2921975 \\ \mathrm{~F} & -4.9111995 & -1.7854413 & 2.3039323 \\ \mathrm{~F} & 3.1358297 & -1.4174834 & 3.7183225 \\ \mathrm{~F} & -3.4319664 & 5.4228008 & -0.0012704 \\ \mathrm{~F} & -5.9069295 & -3.0498223 & 0.0774186 \\ \mathrm{~S} & 0.0537332 & -0.0899469 & -1.8589615 \\ \mathrm{C} & 1.0240729 & -1.5823687 & -1.7362352 \\ \mathrm{C} & 2.0651631 & -1.7758830 & -0.8200767 \\ \mathrm{C} & 0.6860009 & -2.5881982 & -2.6500449 \\ \mathrm{C} & 2.7605223 & -2.9832816 & -0.8216963 \\ \mathrm{H} & 2.3471839 & -0.9914607 & -0.1329537 \\ \mathrm{C} & 1.3788856 & -3.7978617 & -2.6306259 \\ \mathrm{H} & -0.1117102 & -2.4222550 & -3.3652300 \\ \mathrm{C} & 2.4162978 & -3.9962088 & -1.7182579 \\ \mathrm{H} & 3.5738120 & -3.1291280 & -0.1173397 \\ \mathrm{H} & 1.1120071 & -4.5788273 & -3.3358648 \\ \mathrm{H} & 2.9589806 & -4.9366369 & -1.7088652 \\ \mathrm{C} & 0.9849587 & 2.0002443 & -3.3724971 \\ \mathrm{C} & 1.2845031 & 1.3757555 & -2.0073932 \\ \mathrm{H} & -0.0695780 & 2.2689775 & -3.4689247 \\ \mathrm{H} & 1.2464558 & 1.3107858 & -4.1805333 \\ \mathrm{H} & 0.9957210 & 2.0387466 & -1.1917800 \\ \mathrm{C} & 2.6900622 & 0.9202283 & -1.8702730 \\ \mathrm{C} & 3.5140011 & 1.3313424 & -0.8901301 \\ \mathrm{H} & 3.0401837 & 0.2293828 & -2.6342800 \\ \mathrm{H} & 3.1383681 & 2.0516497 & -0.1658928 \\ \mathrm{C} & 4.8872855 & 0.8817897 & -0.6666779 \\ \mathrm{C} & 5.6351383 & 1.4826450 & 0.3620290 \\ \mathrm{C} & 5.4827378 & -0.1580544 & -1.4066851 \\ \mathrm{C} & 6.9375532 & 1.0706129 & 0.6370812 \\ \mathrm{H} & 5.1815999 & 2.2776437 & 0.9486785 \\ \mathrm{C} & 6.7821940 & -0.5695625 & -1.1311874 \\ \mathrm{H} & 4.9227806 & -0.6546942 & -2.1935262 \\ \mathrm{H} & 7.5167354 & 0.0433872 & -0.1098209 \\ \mathrm{H} & 7.4987950 & 1.5482952 & 1.4350780 \\ & 7.2258860 & -1.3750115 & -1.7097125 \\ & 1.5910395 & 2.9059097 & -3.4784198\end{array}$

$\mathbf{E a}^{+}$(site 4-to-1 adduct of $\mathbf{1 a}$ and $\mathbf{B}^{+}$) 41 Energy $=-775.0315457612$

$\begin{array}{llll}\mathrm{H} & 0.4171335 & 1.1903832 & -0.4481290 \\ \mathrm{C} & 0.5305664 & 0.1867034 & -0.8765686\end{array}$




$\begin{array}{lrrr}\mathrm{C} & 1.6700993 & -0.5473694 & -0.2381606 \\ \mathrm{H} & -0.4158457 & -0.3391471 & -0.6929979 \\ \mathrm{H} & 0.6714888 & 0.2811669 & -1.9572675 \\ \mathrm{H} & 1.6055284 & -0.7041289 & 0.8406655 \\ \mathrm{C} & 2.7352736 & -1.0195199 & -0.8945714 \\ \mathrm{C} & 3.8422430 & -1.7938206 & -0.2454660 \\ \mathrm{H} & 2.8066834 & -0.8722856 & -1.9727218 \\ \mathrm{H} & 3.7307084 & -1.7533498 & 0.8421459 \\ \mathrm{C} & 5.2258337 & -1.3027526 & -0.6164371 \\ \mathrm{C} & 6.1780242 & -1.0818100 & 0.3860454 \\ \mathrm{C} & 5.5825957 & -1.0748612 & -1.9529082 \\ \mathrm{C} & 7.4573966 & -0.6269443 & 0.0628471 \\ \mathrm{H} & 5.9123354 & -1.2607620 & 1.4250037 \\ \mathrm{C} & 6.8594125 & -0.6198317 & -2.2773155 \\ \mathrm{H} & 4.8624259 & -1.2490242 & -2.7478513 \\ \mathrm{C} & 7.8011969 & -0.3940707 & -1.2697611 \\ \mathrm{H} & 8.1843545 & -0.4539928 & 0.8512576 \\ \mathrm{H} & 7.1194734 & -0.4403997 & -3.3165074 \\ \mathrm{H} & 8.7963792 & -0.0401023 & -1.5225789 \\ \mathrm{C} & 3.7680386 & -3.3612232 & -0.6645222 \\ \mathrm{C} & 2.5100994 & -3.9047698 & -0.1587715 \\ \mathrm{H} & 4.6382195 & -3.8478505 & -0.2223456 \\ \mathrm{H} & 3.8222382 & -3.3971648 & -1.7556763 \\ \mathrm{H} & 2.4815620 & -4.2908987 & 0.8592324 \\ \mathrm{C} & 1.3261755 & -3.7948039 & -0.8533846 \\ \mathrm{C} & 0.1100449 & -4.0948701 & -0.2395072 \\ \mathrm{H} & 1.3486866 & -3.4100798 & -1.8681749 \\ \mathrm{H} & 0.1483371 & -4.4934593 & 0.7735708 \\ \mathrm{C} & -1.1818221 & -3.8885089 & -0.7905222 \\ \mathrm{C} & -2.3123334 & -4.1949983 & 0.0128349 \\ \mathrm{C} & -1.3819984 & -3.3658028 & -2.0971806 \\ \mathrm{C} & -3.5932563 & -3.9843842 & -0.4693825 \\ \mathrm{H} & -2.1553281 & -4.5925656 & 1.0109097 \\ \mathrm{C} & -2.6642160 & -3.1631129 & -2.5714960 \\ \mathrm{H} & -0.5308037 & -3.1285046 & -2.7254973 \\ \mathrm{C} & -3.7689473 & -3.4701532 & -1.7603002 \\ \mathrm{H} & -4.4550298 & -4.2150863 & 0.1481460 \\ \mathrm{H} & -2.8208192 & -2.7674695 & -3.5693242 \\ \mathrm{H} & -4.7723345 & -3.3046370 & -2.1407404\end{array}$

\begin{tabular}{|c|c|c|c|}
\hline \\
\hline \multirow{2}{*}{\multicolumn{3}{|c|}{$\begin{array}{llll}\mathrm{H} & 4.8017481 & 0.9785107 & -2.2933098\end{array}$}} & \\
\hline & & & \\
\hline 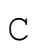 & 3.1544279 & -0.361 & -1.8 \\
\hline $\mathrm{H}$ & 5.0756512 & -0.6867365 & -2.8511942 \\
\hline \multirow{2}{*}{$\begin{array}{l}\mathrm{H} \\
\mathrm{H}\end{array}$} & 5.1970237 & -0.2957908 & -1.1211820 \\
\hline & 2.6289115 & -0.1593358 & -2.8145766 \\
\hline \multirow[t]{2}{*}{$\mathrm{C}$} & 2.5364387 & 0.4225555 & -0.7691415 \\
\hline & 1.3312199 & 1.0154346 & -0.8604013 \\
\hline $\mathrm{H}$ & 3.0852924 & 0.427 & 0.1732035 \\
\hline
\end{tabular}




\begin{tabular}{|c|c|c|c|}
\hline $\mathrm{H}$ & 0.8184586 & 0.9807145 & -1.8226901 \\
\hline C & 0.5819126 & 1.6790709 & 0.2067494 \\
\hline$C$ & -0.6753983 & 2.2325620 & -0.0982866 \\
\hline$\gamma$ & 1.0352442 & 1.7513952 & 1.5391805 \\
\hline 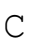 & -1.4534246 & 2.8381214 & 0.8859120 \\
\hline 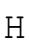 & -1.0400370 & 2.1788110 & -1.1212838 \\
\hline$C$ & 0.2579076 & 2.3553955 & 2.5215028 \\
\hline $\mathrm{H}$ & 1.9989399 & 1.3285095 & 1.8083878 \\
\hline 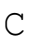 & -0.9903037 & 2.9016763 & 2.2007766 \\
\hline$H$ & -2.4216965 & 3.2568694 & 0.6274911 \\
\hline $\mathrm{H}$ & 0.6235244 & 2.4010620 & 3.5435137 \\
\hline $\mathrm{H}$ & -1.5947903 & 3.3697361 & 2.9721286 \\
\hline C & 2.9962957 & -1.9439093 & -1.5749199 \\
\hline C & 1.5794255 & -2.2926959 & -1.6712649 \\
\hline $\mathrm{H}$ & 3.3959451 & -2.1141242 & -0.5708469 \\
\hline $\mathrm{H}$ & 3.5993591 & -2.4692446 & -2.3178284 \\
\hline $\mathrm{H}$ & 1.1843125 & -2.6006007 & -2.6383143 \\
\hline C & 0.6906044 & -2.0537060 & -0.6470188 \\
\hline$C$ & -0.6839035 & -2.0575417 & -0.8901666 \\
\hline $\mathrm{H}$ & 1.0724835 & -1.7456062 & 0.3206414 \\
\hline $\mathrm{H}$ & -1.0150018 & -2.4225838 & -1.8618334 \\
\hline C & -1.6888577 & -1.5593453 & -0.0227717 \\
\hline $\mathrm{C}$ & -3.0346373 & -1.5716147 & -0.4782923 \\
\hline C & -1.3934790 & -1.0059385 & 1.2526093 \\
\hline C & -4.0447298 & -1.0493231 & 0.3108072 \\
\hline $\mathrm{H}$ & -3.2546653 & -1.9935703 & -1.4546155 \\
\hline C & -2.4088196 & -0.4843902 & 2.0312166 \\
\hline $\mathrm{H}$ & -0.3726742 & -0.9836634 & 1.6150548 \\
\hline C & -3.7309721 & -0.5035750 & 1.5627425 \\
\hline $\mathrm{H}$ & -5.0725523 & -1.0590604 & -0.0358955 \\
\hline $\mathrm{H}$ & -2.1838446 & -0.0530792 & 3.0001385 \\
\hline $\mathrm{H}$ & -4.5232773 & -0.0897074 & 2.1791291 \\
\hline
\end{tabular}

$\left(\mathrm{C}_{6} \mathrm{~F}_{5}\right)_{3} \mathrm{BH}^{-}$borate anion 35

\begin{tabular}{lrrr}
\multicolumn{4}{l}{ Energy $=-2210.269512126$} \\
B & 0.0039517 & -0.0012074 & 0.8299942 \\
C & 0.0323310 & 1.5729667 & 0.3769834 \\
C & 1.3518479 & -0.8138841 & 0.3750220 \\
C & -1.3740181 & -0.7611967 & 0.3761258 \\
C & -0.7718453 & 2.4967224 & 1.0480314 \\
C & 0.8091067 & 2.1274895 & -0.6384579 \\
C & 2.5542498 & -0.5799041 & 1.0453490 \\
C & 1.4416923 & -1.7655477 & -0.6394940 \\
C & -1.7748694 & -1.9179326 & 1.0473262 \\
C & -2.2416993 & -0.3633701 & -0.6394694 \\
C & -0.8111480 & 3.8566203 & 0.7571428 \\
C & 0.8132818 & 3.4821159 & -0.9624445 \\
C & 3.7504716 & -1.2284722 & 0.7558107 \\
C & 2.6119559 & -2.4488777 & -0.9622039 \\
C & -2.9353282 & -2.6284704 & 0.7575489 \\
C & -3.4195618 & -1.0331020 & -0.9618690
\end{tabular}




\begin{tabular}{|c|c|c|c|}
\hline $\mathrm{C}$ & -0.0042242 & 4.3574723 & -0.2587247 \\
\hline $\mathrm{C}$ & 3.7790281 & -2.1792153 & -0.2588996 \\
\hline & -3.7720923 & -2.1772762 & -0.2575912 \\
\hline & -1.5978539 & 2.0745369 & 2.0436516 \\
\hline & 1.6099347 & 1.3418713 & -1.4090238 \\
\hline & 2.6008715 & 0.3478969 & 2.0395066 \\
\hline & 0.3596667 & -2.0664962 & -1.4085126 \\
\hline & -0.9973215 & -2.4235809 & 2.0431338 \\
\hline & -1.9593878 & 0.7220591 & -1.4104031 \\
\hline & -1.6193761 & 4.6974638 & 1.4442150 \\
\hline & 1.5938876 & 3.9551432 & -1.9619885 \\
\hline & 4.8837831 & -0.9514561 & 1.442141 \\
\hline & 2.6301914 & -3.3632686 & -1.96006 \\
\hline & -3.2623993 & -3.7479004 & 1.44488 \\
\hline & -4.2190207 & -0.5915035 & -1.9608010 \\
\hline & -0.0196461 & 5.6728175 & -0.56012 \\
\hline & 4.9245628 & -2.8263391 & -0.55957 \\
\hline & -4.9057720 & -2.8448108 & -0.55823 \\
\hline & 0.0047455 & -0.0024110 & 2 \\
\hline
\end{tabular}

$\left(\mathrm{C}_{6} \mathrm{~F}_{5}\right)_{3} \mathrm{~B}$ borane Lewis acid catalyst 34

Energy $=-2209.516817574$

$\begin{array}{lrrr}\text { B } & 0.0000063 & 0.0013582 & -0.0000364 \\ \text { C } & -0.0000080 & 1.5641699 & 0.0000084 \\ \text { C } & 1.3524692 & -0.7816899 & -0.0032858 \\ \text { C } & -1.3524661 & -0.7816992 & 0.0032394 \\ \text { C } & -0.9452615 & 2.3155953 & 0.7154288 \\ \text { C } & 0.9452759 & 2.3156120 & -0.7154525 \\ \text { C } & 2.4758647 & -0.3418505 & 0.7132955 \\ \text { C } & 1.5275707 & -1.9754051 & -0.7207647 \\ \text { C } & -1.5275554 & -1.9754073 & 0.7207174 \\ \text { C } & -2.4758614 & -0.3418566 & -0.7133362 \\ \text { C } & -0.9562446 & 3.7039037 & 0.7342548 \\ \text { C } & 0.9562510 & 3.7039211 & -0.7342273 \\ \text { C } & 3.6819192 & -1.0290931 & 0.7317163 \\ \text { C } & 2.7228622 & -2.6815690 & -0.7395612 \\ \text { C } & -2.7228583 & -2.6815666 & 0.7395622 \\ \text { C } & -3.6819281 & -1.0290909 & -0.7317160 \\ \text { C } & 0.0000206 & 4.4026124 & 0.0000136 \\ \text { C } & 3.8063244 & -2.2056925 & -0.0038381 \\ \text { C } & -3.8063324 & -2.2056614 & 0.0038698 \\ \text { F } & -1.8839380 & 1.6924057 & 1.4580106 \\ \text { F } & 1.8839388 & 1.6924283 & -1.4580410 \\ \text { F } & 2.4074305 & 0.7825997 & 1.4563558 \\ \text { F } & 0.5175645 & -2.4725503 & -1.4653357 \\ \text { F } & -0.5175297 & -2.4725474 & 1.4652710 \\ \text { F } & -2.4074366 & 0.7825973 & -1.4563808 \\ \text { F } & -1.8730037 & 4.3777899 & 1.4470797 \\ \text { F } & 1.8730240 & 4.3778120 & -1.4470408 \\ \text { F } & 4.7247843 & -0.5749585 & 1.4445503 \\ \text { F } & 2.8477128 & -3.8114417 & -1.4540374 \\ & & & \end{array}$




\begin{tabular}{|c|c|c|c|}
\hline F & -2.8476957 & -3.8114119 & 1.4540684 \\
\hline $\mathrm{F}$ & -4.7248183 & -0.5749393 & -1.4445004 \\
\hline $\mathrm{F}$ & 0.0000033 & 5.7388820 & 0.0000348 \\
\hline $\mathrm{F}$ & 4.9624211 & -2.8755343 & -0.0045252 \\
\hline $\mathrm{F}$ & -4.9624367 & -2.8755118 & 0.0045761 \\
\hline & \multirow{2}{*}{\multicolumn{3}{|c|}{$\mathrm{PhCH}=\mathrm{CHCHMe}-\mathrm{CH}_{2} \mathrm{CH}(\mathrm{CH}=\mathrm{CHPh}) \mathrm{SPh}$}} \\
\hline 53 & & & \\
\hline \multicolumn{4}{|c|}{ Energy $=-1405.269064077$} \\
\hline $\mathrm{C}$ & -0.6895942 & 2.7160669 & -2.5902705 \\
\hline C & -0.7799476 & 1.7428176 & -1.4098825 \\
\hline [ & -0.4550764 & 2.1601779 & -3.5076610 \\
\hline $\mathrm{H}$ & -1.6730923 & 3.1787257 & -2.7349560 \\
\hline $\mathrm{H}$ & -1.0905961 & 2.2714098 & -0.5036296 \\
\hline $\mathrm{C}$ & 0.4335263 & 0.9288832 & -1.1580068 \\
\hline $\mathrm{C}$ & 0.8594504 & 0.6104729 & 0.0766247 \\
\hline $\mathrm{H}$ & 0.9407774 & 0.5420181 & -2.0397031 \\
\hline $\mathrm{H}$ & 0.2914203 & 0.9899011 & 0.9262570 \\
\hline C & 2.0251874 & -0.2010683 & 0.4196568 \\
\hline $\mathrm{C}$ & 2.2216524 & -0.5745110 & 1.7613110 \\
\hline $\mathrm{C}$ & 2.9805469 & -0.6122479 & -0.5288685 \\
\hline $\mathrm{C}$ & 3.3245906 & -1.3360824 & 2.1440798 \\
\hline $\mathrm{H}$ & 1.4920474 & -0.2629919 & 2.5055060 \\
\hline C & 4.0834617 & -1.3672863 & -0.1473390 \\
\hline $\mathrm{H}$ & 2.8755096 & -0.3104869 & -1.5664127 \\
\hline $\mathrm{C}$ & 4.2619850 & -1.7363683 & 1.1903137 \\
\hline $\mathrm{H}$ & 3.4545640 & -1.6132550 & 3.1870527 \\
\hline $\mathrm{H}$ & 4.8190810 & -1.6564619 & -0.8927922 \\
\hline $\mathrm{H}$ & 5.1277663 & -2.3227813 & 1.4853723 \\
\hline S & -2.2111819 & 0.5722211 & -1.8127102 \\
\hline C & -2.2873730 & -0.3339275 & -0.2838249 \\
\hline $\mathrm{C}$ & -3.0367061 & 0.1636731 & 0.7906449 \\
\hline $\mathrm{C}$ & -1.5668683 & -1.5259692 & -0.1345265 \\
\hline C & -3.0625657 & -0.5259836 & 2.0029828 \\
\hline $\mathrm{H}$ & -3.5917074 & 1.0898066 & 0.6721697 \\
\hline C & -1.5979738 & -2.2142749 & 1.0789306 \\
\hline $\mathrm{H}$ & -0.9784256 & -1.8999061 & -0.9658718 \\
\hline C & -2.3429028 & -1.7151196 & 2.1485758 \\
\hline $\mathrm{H}$ & -3.6447950 & -0.1349191 & 2.8328096 \\
\hline $\mathrm{H}$ & -1.0326829 & -3.1350680 & 1.1900826 \\
\hline $\mathrm{H}$ & -2.3637358 & -2.2502525 & 3.0938223 \\
\hline $\mathrm{C}$ & 0.3620147 & 3.8384190 & -2.3914667 \\
\hline C & 0.1124150 & 4.9602898 & -3.4179816 \\
\hline $\mathrm{H}$ & 0.2360182 & 4.2518035 & -1.3820312 \\
\hline $\mathrm{H}$ & 0.8328553 & 5.7736415 & -3.2857019 \\
\hline $\mathrm{H}$ & 0.2142550 & 4.5763793 & -4.4399127 \\
\hline $\mathrm{H}$ & -0.8982351 & 5.3680242 & -3.3053700 \\
\hline $\mathrm{C}$ & 1.7594060 & 3.3036184 & -2.5201345 \\
\hline $\mathrm{C}$ & 2.6592266 & 3.2714534 & -1.5255387 \\
\hline $\mathrm{H}$ & 2.0105326 & 2.8876547 & -3.4977640 \\
\hline $\mathrm{H}$ & 2.3597291 & 3.6598561 & -0.5513212 \\
\hline , & 3.9992882 & 2.6805012 & -1.5670937 \\
\hline
\end{tabular}




$\begin{array}{lllr}\mathrm{C} & 4.7056800 & 2.5201839 & -0.3620380 \\ \mathrm{C} & 4.6036492 & 2.2215955 & -2.7528637 \\ \mathrm{C} & 5.9577841 & 1.9099342 & -0.3342064 \\ \mathrm{H} & 4.2475841 & 2.8569615 & 0.5648671 \\ \mathrm{C} & 5.8551392 & 1.6141975 & -2.7269016 \\ \mathrm{H} & 4.0894053 & 2.3415211 & -3.7025174 \\ \mathrm{C} & 6.5388061 & 1.4514814 & -1.5169332 \\ \mathrm{H} & 6.4758167 & 1.7834638 & 0.6124196 \\ \mathrm{H} & 6.3029334 & 1.2664501 & -3.6543160 \\ \mathrm{H} & 7.5144771 & 0.9740360 & -1.5001867\end{array}$

G $\left(\mathrm{C}_{6} \mathrm{~F}_{5}\right){ }_{3} \mathrm{~B} \cdot \mathrm{CH}_{2}=\mathrm{CHCH}=\mathrm{CHPh}$. PhSH complex 67 Energy $=-3227.453223505$

\begin{tabular}{|c|c|c|c|}
\hline B & 0.5485046 & -0.3325075 & -0.1000840 \\
\hline & 1.2851712 & 0.6639156 & 0.9676359 \\
\hline & -0.8419445 & -1.0458467 & 0.4142338 \\
\hline & 1.5193884 & -1.5485116 & -0.6373557 \\
\hline & 2.6717342 & 0.7291354 & 1.1492553 \\
\hline & 0.5831998 & 1.6161955 & 1.7160049 \\
\hline & -1.6957149 & -1.6699591 & -0.5017082 \\
\hline & -1.2795855 & -1.1487786 & 1.7387696 \\
\hline & 2.1063952 & -1.7199013 & -1.8925822 \\
\hline & 1.7971567 & -2.5884166 & 0.2581850 \\
\hline & 3.3027684 & 1.6164827 & 2.0180438 \\
\hline & 1.1680541 & 2.5303445 & 2.5831282 \\
\hline & -2.9150858 & -2.2547538 & -0.1752967 \\
\hline & -2.4904471 & -1.7262763 & 2.1159681 \\
\hline & 2.8713452 & -2.8306464 & -2.2462936 \\
\hline C & 2.5559840 & -3.7119459 & -0.0467772 \\
\hline C & 2.5483520 & 2.5275407 & 2.7436516 \\
\hline & -3.3275468 & -2.2726208 & 1.1511124 \\
\hline & 3.0967908 & -3.8405716 & -1.3209381 \\
\hline & 3.5107375 & -0.0789055 & 0.4553281 \\
\hline & -0.7720586 & 1.6962223 & 1.6157087 \\
\hline $\mathrm{F}$ & -1.3569829 & -1.7353651 & -1.8199880 \\
\hline $\mathrm{F}^{\prime}$ & -0.5155017 & -0.7045056 & 2.7645580 \\
\hline $\mathrm{F}$ & 1.9895783 & -0.7809016 & -2.8694120 \\
\hline $\mathrm{F}$ & 1.3412345 & -2.5125222 & 1.5350078 \\
\hline $\mathrm{F}$ & 4.6463132 & 1.6210351 & 2.1353640 \\
\hline $\mathrm{F}$ & 0.4152988 & 3.4221397 & 3.2589764 \\
\hline 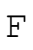 & -3.6960332 & -2.8020177 & -1.1306533 \\
\hline F & -2.8521405 & -1.7727972 & 3.4146399 \\
\hline $\mathrm{F}$ & 3.4033564 & -2.9281284 & -3.4827492 \\
\hline $\mathrm{F}$ & 2.7805437 & -4.6680373 & 0.8779785 \\
\hline $\mathrm{F}$ & 3.1436228 & 3.4127095 & 3.5619961 \\
\hline $\mathrm{F}$ & -4.5032516 & -2.8261703 & 1.4952824 \\
\hline $\mathrm{F}$ & 3.8336563 & -4.9172897 & -1.6473417 \\
\hline C & 0.1794967 & 0.7336013 & -1.4979720 \\
\hline C & -0.8759993 & 1.6161020 & -1.1553840 \\
\hline 11 & 1.1404041 & 1.2279086 & -1.6374729 \\
\hline $\mathrm{H}$ & -0.0770549 & 0.0531323 & -2.3047015 \\
\hline
\end{tabular}




$\begin{array}{lrrr}\mathrm{H} & -0.6457574 & 2.5055697 & -0.5764995 \\ \mathrm{C} & -2.2348000 & 1.3247504 & -1.3634232 \\ \mathrm{C} & -3.2153917 & 2.0363882 & -0.7202704 \\ \mathrm{H} & -2.4861590 & 0.4712861 & -1.9851334 \\ \mathrm{H} & -2.9013130 & 2.8933229 & -0.1240372 \\ \mathrm{C} & -4.6217767 & 1.7399015 & -0.6981822 \\ \mathrm{C} & -5.4752310 & 2.5797686 & 0.0516697 \\ \mathrm{C} & -5.1780874 & 0.6250907 & -1.3679653 \\ \mathrm{C} & -6.8377135 & 2.3198043 & 0.1274550 \\ \mathrm{H} & -5.0504968 & 3.4339334 & 0.5721782 \\ \mathrm{C} & -6.5372646 & 0.3672249 & -1.2850554 \\ \mathrm{H} & -4.5422661 & -0.0364324 & -1.9468766 \\ \mathrm{C} & -7.3702294 & 1.2124298 & -0.5388732 \\ \mathrm{H} & -7.4850849 & 2.9724456 & 0.7044655 \\ \mathrm{H} & -6.9578171 & -0.4918967 & -1.7980892 \\ \mathrm{H} & -8.4339788 & 1.0033613 & -0.4771457 \\ \mathrm{~S} & -0.0348958 & 3.9825126 & -3.0416911 \\ \mathrm{H} & 0.2952193 & 3.1233904 & -4.0227388 \\ \mathrm{C} & 1.4809790 & 3.8673418 & -2.1333154 \\ \mathrm{C} & 2.5673987 & 3.1012796 & -2.5791276 \\ \mathrm{C} & 1.5639403 & 4.5546255 & -0.9138719 \\ \mathrm{C} & 3.7270578 & 3.0315745 & -1.8075117 \\ \mathrm{H} & 2.5073890 & 2.5644807 & -3.5212108 \\ \mathrm{C} & 2.7315981 & 4.4822971 & -0.1569223 \\ \mathrm{H} & 0.7244055 & 5.1495019 & -0.5642606 \\ \mathrm{C} & 3.8168002 & 3.7226781 & -0.5985581 \\ \mathrm{H} & 4.5623368 & 2.4324839 & -2.1577470 \\ \mathrm{H} & 2.7897241 & 5.0231317 & 0.7830399 \\ \mathrm{H} & 4.7221588 & 3.6644623 & -0.0028349 \\ & & & \end{array}$

TSAa (TS for $\mathbf{1 a}$ addition to $\mathrm{B}\left(\mathrm{C}_{6} \mathrm{~F}_{5}\right)_{3}$ into unstable $\mathbf{A a}$ ) 54

$\begin{array}{lrrr}\text { Energy }=-2596.815584045 & \\ \text { B } & 0.8920580 & 0.0199105 & -0.2809017 \\ \text { C } & 1.2924314 & 1.5516643 & 0.0531900 \\ \text { C } & -0.3289118 & -0.6464419 & 0.5616667 \\ \text { C } & 2.1378984 & -1.0363826 & -0.3534962 \\ \text { C } & 2.6108392 & 2.0127829 & 0.1752450 \\ \text { C } & 0.3320482 & 2.5641391 & 0.1912688 \\ \text { C } & -0.8963974 & -1.8576941 & 0.1510556 \\ \text { C } & -0.8772705 & -0.1445737 & 1.7462897 \\ \text { C } & 2.9608065 & -1.3119738 & -1.4502977 \\ \text { C } & 2.4711294 & -1.7688255 & 0.7941237 \\ \text { C } & 2.9496730 & 3.3434450 & 0.4139633 \\ \text { C } & 0.6229452 & 3.9019012 & 0.4226660 \\ \text { C } & -1.9633584 & -2.4831520 & 0.7870010 \\ \text { C } & -1.9498237 & -0.7309342 & 2.4143187 \\ \text { C } & 3.9762304 & -2.2640337 & -1.4430870 \\ \text { C } & 3.4806364 & -2.7248977 & 0.8494459 \\ \text { C } & 1.9506364 & 4.3000777 & 0.5369894 \\ \text { C } & -2.5105431 & -1.9052474 & 1.9258977 \\ \text { C } & 4.2371897 & -2.9866850 & -0.2856646\end{array}$




$\begin{array}{lrrr}\text { F } & 3.6647053 & 1.1680999 & 0.0729458 \\ \text { F } & -0.9884393 & 2.2617309 & 0.1042437 \\ \text { F } & -0.4109502 & -2.5024760 & -0.9450865 \\ \text { F } & -0.3440115 & 0.9421874 & 2.3531426 \\ \text { F } & 2.8349408 & -0.6212682 & -2.6122622 \\ \text { F } & 1.8277005 & -1.5391641 & 1.9673957 \\ \text { F } & 4.2411479 & 3.7102293 & 0.5253677 \\ \text { F } & -0.3630690 & 4.8115791 & 0.5442243 \\ \mathrm{~F} & -2.4718698 & -3.6359641 & 0.3060541 \\ \mathrm{~F} & -2.4353325 & -0.1817846 & 3.5453075 \\ \mathrm{~F} & 4.7208622 & -2.4787388 & -2.5462523 \\ \mathrm{~F} & 3.7378433 & -3.3878020 & 1.9944088 \\ \mathrm{~F} & 2.2596054 & 5.5862270 & 0.7638358 \\ \mathrm{~F} & -3.5479109 & -2.4822640 & 2.5539341 \\ \mathrm{~F} & 5.2160846 & -3.9061459 & -0.2593944 \\ \mathrm{C} & 0.2550847 & 0.1492283 & -2.1397067 \\ \mathrm{C} & -1.0439590 & 0.6688396 & -2.1315336 \\ \mathrm{H} & 1.0254237 & 0.8032584 & -2.5328846 \\ \mathrm{H} & 0.3512209 & -0.8987227 & -2.4104932 \\ \mathrm{H} & -1.1714254 & 1.7481862 & -2.1568639 \\ \mathrm{C} & -2.2199326 & -0.0949346 & -1.9922641 \\ \mathrm{C} & -3.4132429 & 0.5127563 & -1.6983933 \\ \mathrm{H} & -2.1474665 & -1.1757253 & -2.0577836 \\ \mathrm{H} & -3.4228460 & 1.6020639 & -1.6636801 \\ \mathrm{C} & -4.6556212 & -0.1355897 & -1.3676313 \\ \mathrm{C} & -5.7642765 & 0.6700408 & -1.0261388 \\ \mathrm{C} & -4.7994228 & -1.5414191 & -1.3313102 \\ \mathrm{C} & -6.9734100 & 0.0933405 & -0.6566986 \\ \mathrm{H} & -5.6585853 & 1.7513162 & -1.0524671 \\ \mathrm{C} & -6.0069758 & -2.1124531 & -0.9597895 \\ \mathrm{H} & -3.9640594 & -2.1817749 & -1.5937014 \\ \mathrm{C} & -7.0960317 & -1.2984459 & -0.6203531 \\ \mathrm{H} & -7.8182812 & 0.7223232 & -0.3943777 \\ \mathrm{H} & -6.1086227 & -3.1928290 & -0.9310483 \\ \mathrm{H} & -8.0381590 & -1.7520757 & -0.3274936\end{array}$

TSABa (TS for 1-protonation of 1a)

67

$\begin{array}{lrrr}\text { Energy }=-3227.441130435 \\ \text { B } & -0.5440680 & 0.0477167 & 0.4580340 \\ \text { C } & 0.0732449 & -0.2300726 & 1.9396017 \\ \text { C } & -1.7425738 & 1.1649401 & 0.3627582 \\ \text { C } & -1.1326714 & -1.2700948 & -0.3196330 \\ \text { C } & 0.4807879 & -1.4842038 & 2.4017754 \\ \text { C } & 0.4304495 & 0.8306534 & 2.7831680 \\ \text { C } & -2.0832441 & 1.7225791 & -0.8730943 \\ \text { C } & -2.5758294 & 1.5585870 & 1.4141010 \\ \text { C } & -0.7890049 & -1.7665649 & -1.5744966 \\ \text { C } & -2.2084175 & -1.9390680 & 0.2769692 \\ \text { C } & 1.1708154 & -1.6844470 & 3.5951593 \\ \text { C } & 1.1131276 & 0.6762301 & 3.9827360 \\ \text { C } & -3.1208626 & 2.6265296 & -1.0693491\end{array}$




\begin{tabular}{|c|c|c|c|}
\hline C & -3.6288374 & 2.4593578 & 1.2648628 \\
\hline & -1.4437946 & -2.8279416 & -2.1988381 \\
\hline & -2.8876124 & -3.0046137 & -0.2990298 \\
\hline & 1.4911623 & -0.5968897 & 4.3960172 \\
\hline & -3.9032499 & 3.0040274 & 0.0161185 \\
\hline & -2.5032563 & -3.4555367 & -1.5586062 \\
\hline & 0.2363563 & -2.6101932 & 1.6847117 \\
\hline $\mathrm{E}^{\prime}$ & 0.1397033 & 2.1075111 & 2.4328814 \\
\hline 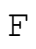 & -1.3859964 & 1.3687203 & -1.9870824 \\
\hline$z^{\prime}$ & -2.4111328 & 1.0622454 & 2.6623356 \\
\hline $\mathrm{H}^{\prime}$ & 0.2521323 & -1.2494986 & -2.2773633 \\
\hline & -2.6367248 & -1.5458898 & 1.5021165 \\
\hline 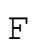 & 1.5408091 & -2.9253298 & 3.9726059 \\
\hline$F$ & 1.4257101 & 1.7462051 & 4.7430485 \\
\hline $\mathrm{F}^{\prime}$ & -3.3793208 & 3.1297051 & -2.2936412 \\
\hline$F$ & -4.3905251 & 2.8049266 & 2.3229841 \\
\hline $\mathrm{F}$ & -1.0500884 & -3.2512656 & -3.4184348 \\
\hline$F$ & -3.9114774 & -3.6029699 & 0.3428555 \\
\hline F & 2.1601251 & -0.7694978 & 5.5496806 \\
\hline 7 & -4.9150629 & 3.8750245 & -0.1419312 \\
\hline 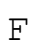 & -3.1462051 & -4.4818538 & -2.1426641 \\
\hline S & 1.0335257 & 0.9971690 & -0.3609803 \\
\hline C & 2.4688890 & 0.0630198 & 0.0938014 \\
\hline $\mathrm{C}$ & 3.5429112 & 0.7677758 & 0.6537747 \\
\hline $\mathrm{C}$ & 2.5584082 & -1.3253182 & -0.0794445 \\
\hline$C$ & 4.6952721 & 0.0869491 & 1.0366171 \\
\hline $\mathrm{H}$ & 3.4676342 & 1.8431171 & 0.7846772 \\
\hline$C$ & 3.7095159 & -1.9999343 & 0.3171474 \\
\hline$H$ & 1.7309587 & -1.8741211 & -0.5097440 \\
\hline$C$ & 4.7805383 & -1.2976839 & 0.8741562 \\
\hline $\mathrm{H}$ & 5.5248243 & 0.6401701 & 1.4671882 \\
\hline $\mathrm{H}$ & 3.7689863 & -3.0780661 & 0.1903071 \\
\hline $\mathrm{H}$ & 5.6767378 & -1.8284837 & 1.1796170 \\
\hline C & -0.6008551 & -0.3033079 & -5.6697166 \\
\hline$C$ & -0.0648322 & 0.6499663 & -4.8676370 \\
\hline $\mathrm{H}$ & -0.0731274 & -1.2338526 & -5.8611936 \\
\hline+ & -1.5644264 & -0.1680946 & -6.1499124 \\
\hline 1 & -0.6053919 & 1.5743444 & -4.6774936 \\
\hline $\mathcal{C}$ & 1.2019948 & 0.4734868 & -4.2579152 \\
\hline C & 1.7571406 & 1.3833678 & -3.3287042 \\
\hline H & 1.7207632 & -0.4618088 & -4.4607382 \\
\hline $\mathrm{H}$ & 1.3320017 & 2.3895376 & -3.3866594 \\
\hline $\mathrm{C}$ & 3.2074446 & 1.3724548 & -2.9540445 \\
\hline $\mathrm{C}$ & 3.7862643 & 2.5666312 & -2.5066592 \\
\hline $\mathrm{C}$ & 3.9771778 & 0.2018203 & -2.9610976 \\
\hline $\mathrm{C}$ & 5.1204934 & 2.5989624 & -2.1015684 \\
\hline $\mathrm{H}$ & 3.1872514 & 3.4738173 & -2.4781049 \\
\hline C & 5.3076942 & 0.2331748 & -2.5526562 \\
\hline $\mathrm{H}$ & 3.5380683 & -0.7433571 & -3.2674448 \\
\hline $\mathrm{C}$ & 5.8839080 & 1.4315111 & -2.1229833 \\
\hline$H$ & 5.5573240 & 3.5331555 & -1.7603209 \\
\hline & 5.8913857 & -0.6826563 & -2.554305 \\
\hline
\end{tabular}




$\begin{array}{llll}\mathrm{H} & 6.9189906 & 1.4504641 & -1.7951968 \\ \mathrm{H} & 1.2247873 & 1.0123544 & -2.2824108\end{array}$

TSAB (TS for 4-protonation of 1a)

67 Energy $=-3227.453824217$

\begin{tabular}{|c|c|c|c|}
\hline B & -1.2578677 & 0.1937563 & 0.5416003 \\
\hline & -0.6952050 & -1.1118143 & 1.3297214 \\
\hline & -1.8018163 & 1.4329267 & 1.4583542 \\
\hline & -2.4270651 & -0.0901730 & -0.5638101 \\
\hline & -0.8181535 & -2.4212944 & 0.8549834 \\
\hline & 0.1402085 & -0.9865549 & 2.4475130 \\
\hline & -1.8991161 & 2.7163434 & 0.9131911 \\
\hline & -2.3111063 & 1.3198890 & 2.755113 \\
\hline & -2.4585159 & 0.2429888 & -1.916223 \\
\hline & -3.6198572 & -0.6514361 & -0.09129 \\
\hline & -0.1906031 & -3.5190266 & 1.439081 \\
\hline & 0.7792321 & -2.0542121 & 3.065035 \\
\hline & -2.4165524 & 3.8170177 & 1.5860830 \\
\hline & -2.8432610 & 2.3936837 & 3.465919 \\
\hline & -3.5608280 & 0.0272576 & -2.7432667 \\
\hline & -4.7427860 & -0.8848634 & -0.8740525 \\
\hline & 0.6139952 & -3.3378781 & 2.5559087 \\
\hline & -2.8935787 & 3.6539599 & 2.8820198 \\
\hline & -4.7147716 & -0.5408359 & -2.221904 \\
\hline & -1.5546757 & -2.6983639 & -0.24885 \\
\hline & 0.4049930 & 0.2377918 & 2.9668 \\
\hline & -1.4764922 & 2.9366969 & -0.3609353 \\
\hline & -2.3250107 & 0.1304029 & 3.4001184 \\
\hline & -1.3872863 & 0.8068961 & -2.5289826 \\
\hline & -3.7120053 & -1.0120890 & 1.2128623 \\
\hline & -0.3470157 & -4.7536372 & 0.9209965 \\
\hline & 1.5744525 & -1.8572830 & 4.136183 \\
\hline & -2.4665555 & 5.0287053 & 0.99774 \\
\hline & -3.3135269 & 2.2210963 & 4.717737 \\
\hline & -3.5136357 & 0.3708980 & -4.0465937 \\
\hline & -5.8522126 & -1.4394954 & -0.3461584 \\
\hline & 1.2334120 & -4.3837563 & 3.1292224 \\
\hline & -3.4015101 & 4.6987325 & 3.5578051 \\
\hline & -5.7872645 & -0.7530077 & -3.0038773 \\
\hline & 0.5466272 & 0.8688770 & -0.1488222 \\
\hline & 1.4368719 & -0.5864404 & -0.6638407 \\
\hline & 2.5866775 & -0.9413453 & 0.0507141 \\
\hline 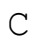 & 1.0120168 & -1.3613583 & -1.7483346 \\
\hline 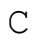 & 3.3016705 & -2.0816271 & -0.3121294 \\
\hline $\mathrm{H}$ & 2.9109248 & -0.3279175 & 0.8852390 \\
\hline C & 1.7344998 & -2.4994370 & -2.1054002 \\
\hline & 0.1232441 & -1.0825631 & -2.3018765 \\
\hline & 2.8747816 & -2.8636732 & -1.3867988 \\
\hline & 4.1922814 & -2.3567466 & 0.2444983 \\
\hline & 1.4020912 & -3.1034965 & -2.944356 \\
\hline & 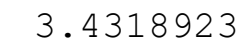 & - & 7 \\
\hline
\end{tabular}




$\begin{array}{lrrr}\mathrm{C} & 1.2228625 & 2.1526870 & -2.8135581 \\ \mathrm{C} & 2.5768223 & 2.3678585 & -2.5789996 \\ \mathrm{H} & 0.9359994 & 1.2829197 & -3.4023347 \\ \mathrm{H} & 0.5591361 & 3.0116066 & -2.8825633 \\ \mathrm{H} & 2.8940376 & 3.3359273 & -2.1914449 \\ \mathrm{C} & 3.5669569 & 1.3617660 & -2.6790335 \\ \mathrm{C} & 4.8594428 & 1.5856694 & -2.2938725 \\ \mathrm{H} & 3.2593764 & 0.3861208 & -3.0486354 \\ \mathrm{H} & 5.1106724 & 2.5823568 & -1.9309848 \\ \mathrm{C} & 5.9407470 & 0.6268305 & -2.2913725 \\ \mathrm{C} & 7.2130335 & 1.0448859 & -1.8459379 \\ \mathrm{C} & 5.7758781 & -0.7162592 & -2.6954844 \\ \mathrm{C} & 8.2822423 & 0.1560779 & -1.8049753 \\ \mathrm{H} & 7.3478566 & 2.0766758 & -1.5315877 \\ \mathrm{C} & 6.8443038 & -1.6005851 & -2.6520303 \\ \mathrm{H} & 4.8067813 & -1.0666152 & -3.0348374 \\ \mathrm{C} & 8.1008050 & -1.1696310 & -2.2073334 \\ \mathrm{H} & 9.2549794 & 0.4928263 & -1.4593193 \\ \mathrm{H} & 6.7047287 & -2.6318164 & -2.9627230 \\ \mathrm{H} & 8.9327532 & -1.8669696 & -2.1745832 \\ \mathrm{H} & 0.7919132 & 1.6496375 & -1.5774681\end{array}$

TSBEa $^{+}$(TS for 4-to-1 addition of $1 \mathbf{a}$ and $\mathbf{B}^{+}$) 41 Energy $=-775.0251638862$

$\begin{array}{lrrr}\mathrm{H} & -1.1624445 & 2.6280971 & 2.2376048 \\ \mathrm{C} & -1.0826228 & 2.1873020 & 1.2358188 \\ \mathrm{C} & -0.1204642 & 1.0497472 & 1.2417778 \\ \mathrm{H} & -2.0898918 & 1.8309981 & 0.9780173 \\ \mathrm{H} & -0.7909265 & 2.9650791 & 0.5254373 \\ \mathrm{H} & -0.3310843 & 0.2341208 & 1.9333071 \\ \mathrm{C} & 1.0001922 & 0.9821678 & 0.4884843 \\ \mathrm{C} & 1.9051936 & -0.1445984 & 0.5466000 \\ \mathrm{H} & 1.2291757 & 1.7953468 & -0.1969581 \\ \mathrm{H} & 1.7125107 & -0.8390259 & 1.3613439 \\ \mathrm{C} & 3.3344960 & 0.0056368 & 0.2281865 \\ \mathrm{C} & 4.2609398 & -0.8386800 & 0.8681322 \\ \mathrm{C} & 3.8058593 & 0.9641491 & -0.6878087 \\ \mathrm{C} & 5.6237852 & -0.7130136 & 0.6186964 \\ \mathrm{H} & 3.9016313 & -1.5854785 & 1.5715102 \\ \mathrm{C} & 5.1682843 & 1.0835286 & -0.9397914 \\ \mathrm{H} & 3.1084595 & 1.6146193 & -1.2065771 \\ \mathrm{C} & 6.0811045 & 0.2478518 & -0.2874751 \\ \mathrm{H} & 6.3292046 & -1.3622857 & 1.1282824 \\ \mathrm{H} & 5.5222395 & 1.8286254 & -1.6458057 \\ \mathrm{H} & 7.1440955 & 0.3445972 & -0.4871732 \\ \mathrm{C} & 1.3728956 & -1.3755934 & -0.9880049 \\ \mathrm{C} & 0.1134122 & -1.8012909 & -0.5572420 \\ \mathrm{H} & 2.2062937 & -2.0663172 & -0.9267907 \\ \mathrm{H} & 1.4319940 & -0.6352203 & -1.7810067 \\ \mathrm{H} & 0.0479004 & -2.6777071 & 0.0855820 \\ \mathrm{C} & -1.0525671 & -1.0482353 & -0.7588657 \\ & & & \\ & & & \end{array}$




$\begin{array}{lrrr}\mathrm{C} & -2.2268836 & -1.3610220 & -0.1070137 \\ \mathrm{H} & -1.0006615 & -0.1867668 & -1.4184931 \\ \mathrm{H} & -2.2193737 & -2.2401316 & 0.5369059 \\ \mathrm{C} & -3.4614323 & -0.6339731 & -0.1604125 \\ \mathrm{C} & -4.5395591 & -1.0829355 & 0.6384844 \\ \mathrm{C} & -3.6400011 & 0.5195737 & -0.9629617 \\ \mathrm{C} & -5.7501466 & -0.4038582 & 0.6390773 \\ \mathrm{H} & -4.4053680 & -1.9684390 & 1.2535321 \\ \mathrm{C} & -4.8524134 & 1.1910050 & -0.9601435 \\ \mathrm{H} & -2.8303944 & 0.8769669 & -1.5906395 \\ \mathrm{C} & -5.9082830 & 0.7334461 & -0.1596691 \\ \mathrm{H} & -6.5711168 & -0.7546250 & 1.2560916 \\ \mathrm{H} & -4.9853233 & 2.0722155 & -1.5796085 \\ \mathrm{H} & -6.8549021 & 1.2653421 & -0.1616303\end{array}$

TSBE$^{+}$(TS for 4-to-3 addition of $\mathbf{1 a}$ and $\mathbf{B}^{+}$)

41

Energy $=-775.0325863372$

$\begin{array}{lrrr}\mathrm{H} & 5.4906758 & 1.2359429 & -0.4894667 \\ \mathrm{C} & 5.1440479 & 0.3480937 & 0.0590830 \\ \mathrm{C} & 3.7745258 & 0.0065197 & -0.4527964 \\ \mathrm{H} & 5.8667931 & -0.4498323 & -0.1214565 \\ \mathrm{H} & 5.1295721 & 0.5983356 & 1.1232474 \\ \mathrm{H} & 3.7295958 & -0.4328253 & -1.4462686 \\ \mathrm{C} & 2.6490249 & 0.7618490 & -0.0452975 \\ \mathrm{C} & 1.4708294 & 0.7535338 & -0.7509336 \\ \mathrm{H} & 2.7297556 & 1.3272557 & 0.8809277 \\ \mathrm{H} & 1.4500006 & 0.1882176 & -1.6815355 \\ \mathrm{C} & 0.2554899 & 1.4643261 & -0.4317385 \\ \mathrm{C} & -0.8234021 & 1.3906081 & -1.3398927 \\ \mathrm{C} & 0.0914681 & 2.2210282 & 0.7504560 \\ \mathrm{C} & -2.0148684 & 2.0590551 & -1.0877292 \\ \mathrm{H} & -0.7071648 & 0.8055027 & -2.2479080 \\ \mathrm{C} & -1.1010118 & 2.8851422 & 0.9994755 \\ \mathrm{H} & 0.9008323 & 2.2859795 & 1.4708960 \\ \mathrm{C} & -2.1569779 & 2.8082284 & 0.0823702 \\ \mathrm{H} & -2.8349507 & 1.9919659 & -1.7953360 \\ \mathrm{H} & -1.2163875 & 3.4635054 & 1.9110403 \\ \mathrm{H} & -3.0881582 & 3.3288759 & 0.2845756 \\ \mathrm{C} & 3.5642060 & -1.9028235 & 0.5676650 \\ \mathrm{C} & 2.3887475 & -2.3672073 & 0.0053137 \\ \mathrm{H} & 3.5450252 & -1.4479652 & 1.5536500 \\ \mathrm{H} & 4.5167445 & -2.2999581 & 0.2357070 \\ \mathrm{H} & 2.4330823 & -2.9624464 & -0.9052534 \\ \mathrm{C} & 1.1259390 & -1.9297910 & 0.4498180 \\ \mathrm{C} & -0.0210244 & -2.1386550 & -0.2810036 \\ \mathrm{H} & 1.0870859 & -1.3513329 & 1.3691221 \\ \mathrm{H} & 0.0644774 & -2.7282311 & -1.1935186 \\ \mathrm{C} & -1.3201205 & -1.6018918 & 0.0049313 \\ \mathrm{C} & -2.3589995 & -1.8160135 & -0.9312462 \\ \mathrm{C} & -1.5930028 & -0.8300141 & 1.1594813 \\ \mathrm{C} & -3.6202254 & -1.2745863 & -0.7258894\end{array}$




$\begin{array}{lrrr}\mathrm{H} & -2.1523312 & -2.4065720 & -1.8196556 \\ \mathrm{C} & -2.8548856 & -0.2962135 & 1.3610769 \\ \mathrm{H} & -0.8150991 & -0.6559109 & 1.8947199 \\ \mathrm{C} & -3.8692982 & -0.5115793 & 0.4192772 \\ \mathrm{H} & -4.4091485 & -1.4402969 & -1.4526355 \\ \mathrm{H} & -3.0560585 & 0.2972269 & 2.2468603 \\ \mathrm{H} & -4.8546610 & -0.0850168 & 0.5810488\end{array}$

$\mathbf{T S B z}^{+}$(TS for E-to-Z rotation of $\mathbf{B}^{+}$)

21

Energy $=-387.6866499990$

$\begin{array}{lrrr}\mathrm{C} & 4.1372421 & 0.5821867 & -0.1509478 \\ \mathrm{C} & 2.8256610 & -0.0736775 & -0.4455237 \\ \mathrm{H} & 4.9585060 & -0.0149915 & -0.5661097 \\ \mathrm{H} & 4.2954320 & 0.7049843 & 0.9237070 \\ \mathrm{H} & 2.5793926 & -0.2316337 & -1.4948252 \\ \mathrm{C} & 1.9521702 & -0.4617094 & 0.4913225 \\ \mathrm{C} & 0.6748023 & -1.1152418 & 0.2259773 \\ \mathrm{H} & 2.1760199 & -0.3203085 & 1.5533237 \\ \mathrm{H} & 0.6663228 & -2.2040826 & 0.1345232 \\ \mathrm{C} & -0.5502758 & -0.4730442 & 0.1160692 \\ \mathrm{C} & -1.7258006 & -1.2620695 & -0.1072182 \\ \mathrm{C} & -0.6580006 & 0.9518143 & 0.2160268 \\ \mathrm{C} & -2.9529644 & -0.6464643 & -0.2230883 \\ \mathrm{H} & -1.6257797 & -2.3405529 & -0.1815259 \\ \mathrm{C} & -1.8916563 & 1.5500695 & 0.0991500 \\ \mathrm{H} & 0.2410704 & 1.5364282 & 0.3802457 \\ \mathrm{C} & -3.0324537 & 0.7530552 & -0.1191711 \\ \mathrm{H} & -3.8516934 & -1.2285467 & -0.3927645 \\ \mathrm{H} & -1.9936287 & 2.6269741 & 0.1725904 \\ \mathrm{H} & -4.0013362 & 1.2348419 & -0.2107235 \\ \mathrm{H} & 4.1878871 & 1.5657543 & -0.6336060\end{array}$

TSG3 (TS for 4,3-thiolation of $\mathbf{A a}$ )

67

\begin{tabular}{lrrr}
\multicolumn{4}{l}{ Energy $=-3227.390446945$} \\
B & 0.3924880 & -0.4815132 & 0.0247428 \\
C & 1.2780711 & 0.5777020 & 0.9100007 \\
C & -1.1197158 & -0.8250133 & 0.5519877 \\
C & 1.1403397 & -1.9201169 & -0.2245332 \\
C & 2.6756340 & 0.5033961 & 0.9846778 \\
C & 0.7519887 & 1.7127438 & 1.5390280 \\
C & -2.0553653 & -1.4186245 & -0.3032083 \\
C & -1.5907901 & -0.6553990 & 1.8588491 \\
C & 1.6534827 & -2.4301875 & -1.4167457 \\
C & 1.2676749 & -2.7911315 & 0.8625631 \\
C & 3.4753321 & 1.4247169 & 1.6559573 \\
C & 1.5095342 & 2.6575508 & 2.2227611 \\
C & -3.3648805 & -1.7262581 & 0.0457321 \\
C & -2.8964460 & -0.9391010 & 2.2539059 \\
C & 2.2231567 & -3.6950558 & -1.5422603 \\
C & 1.8314703 & -4.0597663 & 0.7903543
\end{tabular}




\begin{tabular}{|c|c|c|c|}
\hline C & 2.8890381 & 2.5115552 & 2.2894575 \\
\hline C & -3.7995916 & -1.4659183 & 1.3392013 \\
\hline $\mathrm{C}$ & 2.3120310 & -4.5222292 & -0.4298781 \\
\hline $\mathrm{F}$ & 3.3547304 & -0.4899942 & 0.3574216 \\
\hline $\mathrm{F}$ & -0.5813769 & 1.9787152 & 1.4883748 \\
\hline $\mathrm{F}$ & -1.7068139 & -1.7411443 & -1.5818543 \\
\hline $\mathrm{F}$ & -0.7734100 & -0.2250783 & 2.8482126 \\
\hline $\mathrm{F}$ & 1.6421280 & -1.6869269 & -2.5597155 \\
\hline $\mathrm{F}$ & 0.8476169 & -2.3933792 & 2.0894973 \\
\hline $\mathrm{F}$ & 4.8151702 & 1.2858029 & 1.6705910 \\
\hline $\mathrm{F}$ & 0.9214756 & 3.7281051 & 2.7923694 \\
\hline $\mathrm{F}$ & -4.2070628 & -2.2748761 & -0.8507576 \\
\hline $\mathrm{F}$ & -3.2874141 & -0.7252914 & 3.5268383 \\
\hline $\mathrm{F}$ & 2.6935423 & -4.1207460 & -2.7319919 \\
\hline $\mathrm{F}$ & 1.9230567 & -4.8394616 & 1.8859466 \\
\hline $\mathrm{F}$ & 3.6444704 & 3.4266046 & 2.9193827 \\
\hline $\mathrm{F}$ & -5.0615502 & -1.7423298 & 1.7052925 \\
\hline $\mathrm{F}$ & 2.8590155 & -5.7457946 & -0.5297107 \\
\hline C & 0.2332752 & 0.4063744 & -1.5456642 \\
\hline C & -0.6015049 & 1.6964130 & -1.5097648 \\
\hline $\mathrm{H}$ & 1.3231552 & 0.5491902 & -1.5939604 \\
\hline $\mathrm{H}$ & -0.2254240 & -0.4009950 & -2.1228326 \\
\hline $\mathrm{H}$ & -0.4091504 & 2.3416995 & -0.6577144 \\
\hline C & -2.0474774 & 1.4538694 & -1.7049870 \\
\hline C & -2.9731483 & 1.9367990 & -0.8575822 \\
\hline $\mathrm{H}$ & -2.3231905 & 0.7883791 & -2.5209032 \\
\hline $\mathrm{H}$ & -2.6366546 & 2.5684843 & -0.0363276 \\
\hline C & -4.4004862 & 1.6252279 & -0.8589285 \\
\hline C & -5.1721014 & 2.0155838 & 0.2503091 \\
\hline C & -5.0299573 & 0.9107781 & -1.8954160 \\
\hline C & -6.5240184 & 1.6895916 & 0.3329628 \\
\hline $\mathrm{H}$ & -4.6958470 & 2.5676010 & 1.0569402 \\
\hline C & -6.3802150 & 0.5898668 & -1.8146564 \\
\hline $\mathrm{H}$ & -4.4609133 & 0.6041207 & -2.7681722 \\
\hline C & -7.1325872 & 0.9736215 & -0.6991445 \\
\hline $\mathrm{H}$ & -7.1014691 & 1.9926187 & 1.2015333 \\
\hline $\mathrm{H}$ & -6.8507352 & 0.0360913 & -2.6220077 \\
\hline $\mathrm{H}$ & -8.1860198 & 0.7162081 & -0.6383586 \\
\hline S & 0.1571375 & 2.5880993 & -3.0387711 \\
\hline $\mathrm{H}$ & 0.5113707 & 0.8424891 & -2.7844504 \\
\hline C & 1.7338472 & 2.9871844 & -2.3092472 \\
\hline C & 2.8861830 & 2.3041064 & -2.7181274 \\
\hline C & 1.8008644 & 3.9615667 & -1.3056294 \\
\hline C & 4.1091331 & 2.5982301 & -2.1133485 \\
\hline $\mathrm{H}$ & 2.8266424 & 1.5561380 & -3.5031686 \\
\hline C & 3.0265466 & 4.2418898 & -0.7061219 \\
\hline $\mathrm{H}$ & 0.9055763 & 4.4945090 & -1.0004583 \\
\hline C & 4.1794930 & 3.5617024 & -1.1069902 \\
\hline $\mathrm{H}$ & 5.0028360 & 2.0684445 & -2.4278651 \\
\hline $\mathrm{H}$ & 3.0804925 & 4.9949175 & 0.0741463 \\
\hline $\mathrm{H}$ & 5.1311898 & 3.7835022 & -0.6343145 \\
\hline
\end{tabular}




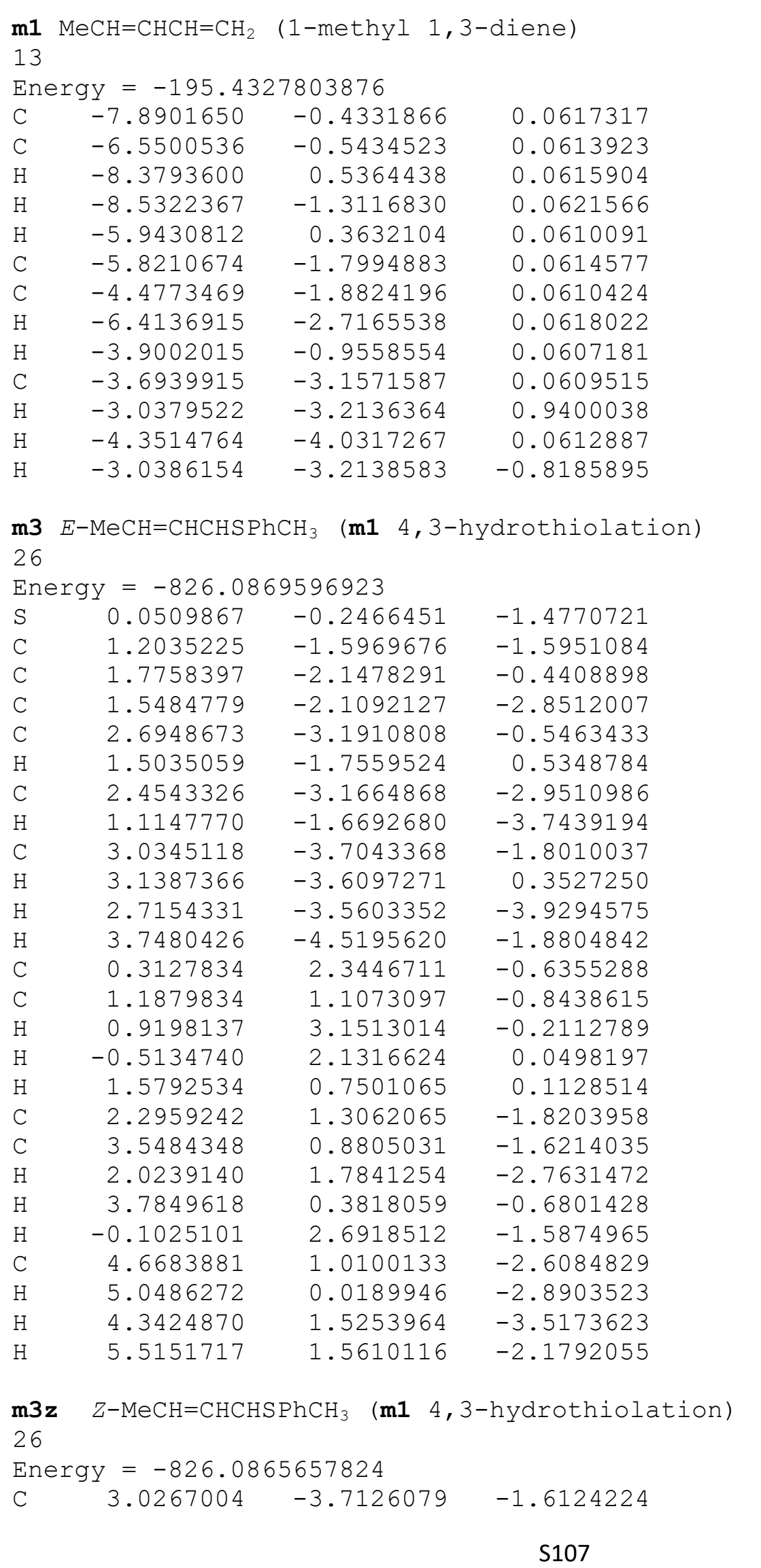




$\begin{array}{rrrr}\text { C } & 2.3467325 & -3.4367284 & -0.4232467 \\ \mathrm{C} & 1.4242825 & -2.3920346 & -0.3719157 \\ \mathrm{C} & 1.1806094 & -1.6136380 & -1.5113925 \\ \mathrm{C} & 1.8588875 & -1.8958884 & -2.7038733 \\ \mathrm{C} & 2.7787067 & -2.9445152 & -2.7519986 \\ \mathrm{~S} & 0.0318744 & -0.2561910 & -1.4333101 \\ \mathrm{C} & 1.1756943 & 1.1083355 & -0.8273551 \\ \mathrm{C} & 2.2233358 & 1.3673434 & -1.8555809 \\ \mathrm{C} & 3.4963557 & 0.9457585 & -1.8232336 \\ \mathrm{C} & 4.1638276 & 0.1359450 & -0.7523107 \\ \mathrm{C} & 0.2891807 & 2.3246276 & -0.5524367 \\ \mathrm{H} & 0.8929319 & -2.1711042 & 0.5492220 \\ \mathrm{H} & 1.6684066 & -1.2873747 & -3.5816901 \\ \mathrm{H} & 2.5348426 & -4.0343281 & 0.4643753 \\ \mathrm{H} & 3.3037894 & -3.1585371 & -3.6787797 \\ \mathrm{H} & 3.7451526 & -4.5265460 & -1.6512319 \\ \mathrm{H} & 0.9026939 & 3.1383812 & -0.1513785 \\ \mathrm{H} & -0.4925761 & 2.0848207 & 0.1746172 \\ \mathrm{H} & 1.6031895 & 0.7333671 & 0.1047159 \\ \mathrm{H} & 1.8810599 & 1.9272098 & -2.7262520 \\ \mathrm{H} & 4.1296104 & 1.2072401 & -2.6714858 \\ \mathrm{H} & -0.1850784 & 2.6765939 & -1.4748682 \\ \mathrm{H} & 5.0746946 & 0.6364844 & -0.4001447 \\ \mathrm{H} & 3.5145284 & -0.0479477 & 0.1064025 \\ \mathrm{H} & 4.4698432 & -0.8390005 & -1.1533628\end{array}$

$\mathbf{m B a}^{+} \mathrm{E}-\mathrm{MeCH}_{2} \mathrm{CHCH}=\mathrm{CH}_{2}{ }^{+}(\mathbf{m} \mathbf{1}$ protonation at site 1$)$ 14 Energy $=-195.8276371601$

$\begin{array}{lrrr}\mathrm{C} & -4.4568185 & -0.6563369 & -0.7110923 \\ \mathrm{C} & -3.2236237 & -1.0438668 & -0.2648745 \\ \mathrm{H} & -5.1933344 & -1.3776164 & -1.0535066 \\ \mathrm{H} & -4.7330508 & 0.3949433 & -0.7360829 \\ \mathrm{H} & -2.9355989 & -2.0904771 & -0.2399995 \\ \mathrm{C} & -2.3287546 & -0.0565697 & 0.1631955 \\ \mathrm{C} & -0.9982283 & -0.2962571 & 0.6938217 \\ \mathrm{H} & -2.6676402 & 0.9795163 & 0.1126876 \\ \mathrm{H} & -0.7047580 & -1.3454097 & 0.6161700 \\ \mathrm{H} & -1.1098713 & -0.0612393 & 1.7741195 \\ \mathrm{C} & 0.0758636 & 0.6764457 & 0.1430302 \\ \mathrm{H} & -0.2378944 & 1.7159315 & 0.2667501 \\ \mathrm{H} & 1.0049536 & 0.5179814 & 0.6934880 \\ \mathrm{H} & 0.2498031 & 0.4783889 & -0.9169865\end{array}$

$\mathbf{m B}^{+} \cdot \mathbf{C}^{-} \quad$ (loose complex of $\mathbf{m B}^{+}$and $\mathbf{C}^{-}$)
60
Energy $=-3035.604530402$
$\begin{array}{llrr}\text { B } & -0.9650161 & 0.3673876 & 0.4183923 \\ \text { C } & -1.1313697 & -1.1401828 & 1.0320435 \\ \text { C } & -2.0954304 & 1.4593182 & 0.9053750 \\ \text { C } & -0.9694645 & 0.4575602 & -1.2201191 \\ \text { C } & -1.0338265 & -2.3297255 & 0.3066097\end{array}$ 


\begin{tabular}{|c|c|c|c|}
\hline $\mathrm{C}$ & -1.2766985 & -1.3336530 & 2.4129314 \\
\hline $\mathrm{C}$ & -1.8957723 & 2.8231243 & 0.6607318 \\
\hline C & -3.3388439 & 1.1581079 & 1.4686002 \\
\hline & 0.0221873 & 0.9452935 & -2.0695807 \\
\hline & -2.1489505 & 0.0895586 & -1.8787142 \\
\hline & -1.0794933 & -3.5998237 & 0.8795463 \\
\hline & -1.3309409 & -2.5779562 & 3.0268527 \\
\hline C & -2.8122708 & 3.8186855 & 0.9819842 \\
\hline $\mathrm{C}$ & -4.2878625 & 2.1220109 & 1.8029514 \\
\hline & -0.1245175 & 1.0492847 & -3.4530669 \\
\hline & -2.3438201 & 0.1777224 & -3.2505904 \\
\hline & -1.2305912 & -3.7296485 & 2.2523913 \\
\hline & -4.0232914 & 3.4649987 & 1.5651365 \\
\hline & -1.3156317 & 0.6645172 & -4.0523307 \\
\hline & -0.8628001 & -2.3209150 & -1.0425267 \\
\hline & -1.3798161 & -0.2657915 & 3.2411280 \\
\hline & -0.7556976 & 3.2424626 & 0.0527589 \\
\hline & -3.7119754 & -0.1225808 & 1.7030247 \\
\hline & 1.2235079 & 1.3525927 & -1.5913792 \\
\hline & -3.1842345 & -0.4081815 & -1.1564212 \\
\hline & -0.9663872 & -4.7025435 & 0.1098040 \\
\hline & -1.4832061 & -2.6830664 & 4.3634375 \\
\hline & -2.5444651 & 5.1154693 & 0.7246046 \\
\hline & -5.4681772 & 1.7622567 & 2.3488246 \\
\hline & 0.8850224 & 1.5247542 & -4.2127424 \\
\hline & -3.5074391 & -0.2078205 & -3.8134437 \\
\hline & -1.2778111 & -4.9452286 & 2.8260322 \\
\hline & -4.9291482 & 4.4065844 & 1.8829596 \\
\hline & -1.4745106 & 0.7594271 & -5.3845147 \\
\hline & 0.6764269 & 1.0221162 & 1.3581511 \\
\hline & 1.9352778 & -0.1511692 & 1.1361008 \\
\hline & 3.0145071 & -0.1222738 & 2.0550730 \\
\hline & 1.9573973 & -1.0993515 & 0.0926465 \\
\hline & 4.0565742 & -1.0358839 & 1.9522715 \\
\hline & 2.9904714 & 0.5918350 & 2.8730502 \\
\hline & 3.0154070 & -1.9836812 & -0.0192976 \\
\hline & 1.1560219 & -1.1214920 & -0.6342229 \\
\hline & 4.0826191 & -1.9506665 & 0.8984429 \\
\hline & 4.8626536 & -1.0155261 & 2.6796008 \\
\hline & 3.0275342 & -2.7035110 & -0.8325943 \\
\hline & 4.8907149 & -2.6706536 & 0.8180063 \\
\hline C & 3.2778424 & 2.8030487 & 0.1341523 \\
\hline 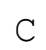 & 4.2439692 & 1.7021611 & 0.1751031 \\
\hline $\mathrm{H}$ & 3.0508229 & 3.1181066 & -0.8871002 \\
\hline $\mathrm{H}$ & 3.5933147 & 3.6479560 & 0.7506177 \\
\hline $\mathrm{H}$ & 4.8854956 & 1.6172167 & 1.0492631 \\
\hline C & 4.4483276 & 0.7939374 & -0.8557469 \\
\hline $\mathrm{C}$ & 5.4260105 & -0.1777687 & -0.7754622 \\
\hline 11 & 3.8236440 & 0.8521599 & -1.7445868 \\
\hline $\mathrm{H}$ & 6.0655223 & -0.1856942 & 0.1041909 \\
\hline $\mathrm{H}$ & 2.3020958 & 2.4450164 & 0.5557250 \\
\hline $\mathrm{C}$ & 5.7853788 & -1.0905851 & -1.8879828 \\
\hline
\end{tabular}




\begin{tabular}{|c|c|c|c|}
\hline $\mathrm{H}$ & 6.0144596 & -2.0968687 & -1.5242630 \\
\hline $\mathrm{H}$ & 5.0064371 & -1.1374527 & -2.6523679 \\
\hline $\mathrm{H}$ & 6.7098281 & -0.7193841 & -2.3576525 \\
\hline $\begin{array}{l}\mathrm{mB}^{+} \\
14\end{array}$ & $\mathrm{E}-\mathrm{MeCH}=\mathrm{CHCHCH}_{3}{ }^{+}$ & \multicolumn{2}{|c|}{ (m1 protonation at site 4$)$} \\
\hline \multicolumn{4}{|c|}{ Energy $=-195.8480527241$} \\
\hline $\mathrm{C}$ & -4.3999222 & 0.3313530 & 0.0112043 \\
\hline $\mathrm{C}$ & -3.1467201 & -0.4153195 & 0.0828504 \\
\hline $\mathrm{H}$ & -5.0138047 & -0.0864826 & -0.8025625 \\
\hline $\mathrm{H}$ & -4.2648614 & 1.4049009 & -0.1181350 \\
\hline $\mathrm{H}$ & -3.2171074 & -1.4965874 & 0.2028185 \\
\hline $\mathrm{C}$ & -1.8802907 & 0.1392374 & 0.0113152 \\
\hline $\mathrm{C}$ & -0.7777236 & -0.6936511 & 0.0950157 \\
\hline $\mathrm{H}$ & -1.7536266 & 1.2120677 & -0.1084421 \\
\hline $\mathrm{H}$ & -0.9604090 & -1.7619259 & 0.2140501 \\
\hline $\mathrm{H}$ & -4.9862750 & 0.1204130 & 0.9201524 \\
\hline C & 0.6146681 & -0.2561729 & 0.0366055 \\
\hline $\mathrm{H}$ & 0.7313145 & 0.8207749 & -0.0813638 \\
\hline $\mathrm{H}$ & 1.1329389 & -0.6071506 & 0.9432474 \\
\hline $\mathrm{H}$ & 1.1192129 & -0.7941222 & -0.7819502 \\
\hline
\end{tabular}

\section{$\mathbf{m B z}^{+} \quad Z-\mathrm{MeCH}=\mathrm{CHCHCH}_{3}{ }^{+}$}

14

Energy $=-195.8438888362$

$\begin{array}{lrrr}\mathrm{C} & -4.4807069 & 0.2161533 & 0.0197075 \\ \mathrm{C} & -3.1635558 & -0.4181760 & 0.0806611 \\ \mathrm{H} & -5.0449361 & -0.2528640 & -0.8016039 \\ \mathrm{H} & -4.4780544 & 1.2984309 & -0.0963945 \\ \mathrm{H} & -3.1736823 & -1.5009979 & 0.2008085 \\ \mathrm{C} & -1.9120293 & 0.1803057 & 0.0045057 \\ \mathrm{C} & -1.7523290 & 1.5462279 & -0.1465175 \\ \mathrm{H} & -1.0310494 & -0.4519722 & 0.0667658 \\ \mathrm{H} & -2.6393934 & 2.1740444 & -0.2081172 \\ \mathrm{H} & -5.0384434 & -0.0685349 & 0.9253540 \\ \mathrm{C} & -0.4628998 & 2.2264263 & -0.2302226 \\ \mathrm{H} & 0.3933119 & 1.5558420 & -0.1620242 \\ \mathrm{H} & -0.4303979 & 2.8067346 & -1.1665518 \\ \mathrm{H} & -0.4195909 & 2.9961060 & 0.5573481\end{array}$

mD MeCH=CHCHMeSPh.B $\left(\mathrm{C}_{6} \mathrm{~F}_{5}\right)_{3}$ complex

60

Energy $=-3035.626140120$

$\begin{array}{lrrr}\text { B } & -0.9230239 & 0.3868983 & 0.4817514 \\ \text { C } & 0.1464606 & 0.5777184 & 1.6712203 \\ \text { C } & -1.6272832 & 1.7396844 & -0.0543849 \\ \text { C } & -1.8856087 & -0.8993330 & 0.4973618 \\ \text { C } & 1.0309434 & -0.4347638 & 2.0636967 \\ \text { C } & 0.2896542 & 1.7633096 & 2.4067463 \\ \text { C } & -0.9161653 & 2.7834070 & -0.6534278 \\ \text { C } & -2.9981010 & 1.9868783 & 0.0778134 \\ \text { C } & -2.6724327 & -1.2101825 & -0.6211329\end{array}$




\begin{tabular}{|c|c|c|c|}
\hline C & -2.0434593 & -1.7835623 & 1.5692721 \\
\hline C & 2.0039747 & -0.2974938 & 3.0437329 \\
\hline 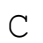 & 1.2471991 & 1.9450447 & 3.4035076 \\
\hline C & -1.4971999 & 3.9446033 & -1.1464032 \\
\hline $\mathrm{C}$ & -3.6235102 & 3.1391468 & -0.3911533 \\
\hline C & -3.4808636 & -2.3329314 & -0.7200289 \\
\hline C & -2.8429137 & -2.9232909 & 1.5143139 \\
\hline $\mathrm{C}$ & 2.1195111 & 0.9121319 & 3.7217762 \\
\hline $\mathrm{C}$ & -2.8706438 & 4.1239983 & -1.0191092 \\
\hline C & -3.5572457 & -3.2098885 & 0.3572681 \\
\hline $\mathrm{F}$ & 0.9661630 & -1.6537345 & 1.4725281 \\
\hline $\mathrm{F}$ & -0.5292410 & 2.8204014 & 2.2050160 \\
\hline $\mathrm{F}$ & 0.4347595 & 2.7099782 & -0.7434731 \\
\hline $\mathrm{F}$ & -3.8066220 & 1.1028008 & 0.7115410 \\
\hline $\mathrm{F}$ & -2.6707505 & -0.3806321 & -1.6932758 \\
\hline $\mathrm{F}$ & -1.4411866 & -1.5532606 & 2.7596713 \\
\hline $\mathrm{F}$ & 2.8243462 & -1.3198204 & 3.3479952 \\
\hline $\mathrm{F}$ & 1.3242906 & 3.1115907 & 4.0690015 \\
\hline $\mathrm{F}$ & -0.7449648 & 4.9033443 & -1.7200740 \\
\hline $\mathrm{F}$ & -4.9505980 & 3.3097156 & -0.2362163 \\
\hline $\mathrm{F}$ & -4.1872759 & -2.5808588 & -1.8383350 \\
\hline $\mathrm{F}$ & -2.9436698 & -3.7402472 & 2.5803962 \\
\hline $\mathrm{F}$ & 3.0445094 & 1.0699973 & 4.6785188 \\
\hline $\mathrm{F}$ & -3.4566174 & 5.2403391 & -1.4793707 \\
\hline $\mathrm{F}$ & -4.3286750 & -4.3057147 & 0.2876342 \\
\hline S & 0.3790613 & -0.0909793 & -1.3829862 \\
\hline C & -0.0617231 & -1.6967285 & -2.0170945 \\
\hline C & 0.0610518 & -2.8704645 & -1.2672961 \\
\hline C & -0.5377335 & -1.7412596 & -3.3309143 \\
\hline C & -0.2983266 & -4.0892801 & -1.8384053 \\
\hline $\mathrm{H}$ & 0.4261694 & -2.8359176 & -0.2505422 \\
\hline C & -0.9011082 & -2.9646215 & -3.8922943 \\
\hline $\mathrm{H}$ & -0.6265503 & -0.8231710 & -3.9022065 \\
\hline C & -0.7838012 & -4.1385244 & -3.1470010 \\
\hline $\mathrm{H}$ & -0.2027988 & -5.0010017 & -1.2563360 \\
\hline $\mathrm{H}$ & -1.2732087 & -2.9976347 & -4.9117478 \\
\hline $\mathrm{H}$ & -1.0687332 & -5.0905780 & -3.5846770 \\
\hline C & 2.8457613 & 0.9641163 & -0.5676762 \\
\hline C & 2.2378627 & -0.3471816 & -1.0598719 \\
\hline $\mathrm{H}$ & 2.3483043 & 1.3395546 & 0.3275699 \\
\hline $\mathrm{H}$ & 2.7983933 & 1.7315670 & -1.3443846 \\
\hline $\mathrm{H}$ & 2.2964857 & -1.1288003 & -0.3023973 \\
\hline C & 2.8221304 & -0.8158519 & -2.3483428 \\
\hline C & 3.2161187 & -2.0765119 & -2.5610594 \\
\hline $\mathrm{H}$ & 2.9063428 & -0.0705391 & -3.1395711 \\
\hline $\mathrm{H}$ & 3.1053177 & -2.8010167 & -1.7532536 \\
\hline $\mathrm{H}$ & 3.8982681 & 0.7779056 & -0.3308613 \\
\hline C & 3.7923709 & -2.5901825 & -3.8422192 \\
\hline $\mathrm{H}$ & 4.7855823 & -3.0270559 & -3.6793213 \\
\hline $\mathrm{H}$ & 3.1595151 & -3.3911575 & -4.2468164 \\
\hline $\mathrm{H}$ & 3.8743679 & -1.7984461 & -4.592515 \\
\hline
\end{tabular}




\begin{tabular}{lrrr}
$\mathbf{m E}^{+}$ & (site 4-to-3 & adduct of $\mathbf{m} 1$ & \multicolumn{1}{l}{ and $\mathbf{m B}^{+}$) } \\
27 & \multicolumn{3}{c}{} \\
Energy $=-391.3069110904$ \\
$\mathrm{H}$ & 0.5702748 & 1.3653392 & -0.5763151 \\
$\mathrm{C}$ & 0.6480501 & 0.3852157 & -1.0632844 \\
$\mathrm{C}$ & 1.6651306 & -0.4705715 & -0.3737856 \\
$\mathrm{H}$ & -0.3486764 & -0.0715125 & -1.0010289 \\
$\mathrm{H}$ & 0.8978298 & 0.5364051 & -2.1173289 \\
$\mathrm{H}$ & 1.4834735 & -0.6859630 & 0.6808236 \\
$\mathrm{C}$ & 2.7671684 & -0.9696534 & -0.9479830 \\
$\mathrm{C}$ & 3.7761106 & -1.8101964 & -0.2396684 \\
$\mathrm{H}$ & 2.9653591 & -0.7691371 & -2.0025227 \\
$\mathrm{H}$ & 3.5564028 & -1.8405718 & 0.8316025 \\
$\mathrm{C}$ & 3.7278584 & -3.3450279 & -0.7946652 \\
$\mathrm{C}$ & 2.5136373 & -3.9229437 & -0.2635556 \\
$\mathrm{H}$ & 4.6216665 & -3.8404401 & -0.4138573 \\
$\mathrm{H}$ & 3.7372277 & -3.2815348 & -1.8850281 \\
$\mathrm{H}$ & 2.5509991 & -4.3789411 & 0.7248441 \\
$\mathrm{C}$ & 1.2670120 & -3.7957938 & -0.8684701 \\
$\mathrm{C}$ & 0.1365170 & -4.1847481 & -0.1827557 \\
$\mathrm{H}$ & 1.1836844 & -3.3592230 & -1.8597254 \\
$\mathrm{H}$ & 0.2691415 & -4.6285133 & 0.8040371 \\
$\mathrm{C}$ & 5.2157707 & -1.3206796 & -0.4600542 \\
$\mathrm{H}$ & 5.3018569 & -0.2937037 & -0.0908089 \\
$\mathrm{H}$ & 5.9306578 & -1.9490756 & 0.0782672 \\
$\mathrm{H}$ & 5.4703532 & -1.3287991 & -1.5248654 \\
$\mathrm{C}$ & -1.2369262 & -4.0350764 & -0.6719021 \\
$\mathrm{H}$ & -1.7308287 & -5.0187997 & -0.6609324 \\
$\mathrm{H}$ & -1.8117522 & -3.4346170 & 0.0498534 \\
$\mathrm{H}$ & -1.2957498 & -3.5921556 & -1.6664277
\end{tabular}

mTSABa (TS for 1-protination of $\mathbf{m} \mathbf{1}$ )

60

Energy $=-3035.588479006$

$\begin{array}{lrrr}\text { B } & -0.2145533 & -0.3973862 & 0.0762630 \\ \text { C } & 1.1031830 & -1.3570308 & 0.0985439 \\ \text { C } & -1.5598757 & -1.0008631 & 0.7911708 \\ \text { C } & -0.6922955 & 0.1236181 & -1.3959315 \\ \text { C } & 1.9753200 & -1.5455197 & -0.9775436 \\ \text { C } & 1.5091872 & -1.9981490 & 1.2775485 \\ \text { C } & -2.6189079 & -0.1458919 & 1.1115550 \\ \text { C } & -1.8262804 & -2.3531244 & 1.0247379 \\ \text { C } & -0.8169766 & 1.4277225 & -1.8684086 \\ \text { C } & -1.1401728 & -0.8521912 & -2.2949509 \\ \text { C } & 3.1384985 & -2.3098053 & -0.9095716 \\ \text { C } & 2.6572845 & -2.7714806 & 1.3894119 \\ \text { C } & -3.8241545 & -0.5614342 & 1.6651358 \\ \text { C } & -3.0212110 & -2.8165514 & 1.5714483 \\ \text { C } & -1.3295488 & 1.7528639 & -3.1240572 \\ \text { C } & -1.6558128 & -0.5793825 & -3.5547972 \\ \text { C } & 3.4850087 & -2.9312452 & 0.2825289 \\ \text { C } & -4.0279903 & -1.9165528 & 1.9003948\end{array}$




$\begin{array}{lrrr} & & & \\ \mathrm{C} & -1.7542894 & 0.7434040 & -3.9769079 \\ \mathrm{~F} & 1.7382353 & -0.9695645 & -2.1838143 \\ \mathrm{~F} & 0.7656018 & -1.8804946 & 2.4044086 \\ \mathrm{~F} & -2.5022874 & 1.1879773 & 0.8698277 \\ \mathrm{~F} & -0.9236322 & -3.3097765 & 0.7075538 \\ \mathrm{~F} & -0.4326116 & 2.4860228 & -1.1124647 \\ \mathrm{~F} & -1.0606012 & -2.1602042 & -1.9459577 \\ \mathrm{~F} & 3.9323171 & -2.4447424 & -1.9902856 \\ \mathrm{~F} & 2.9770767 & -3.3666164 & 2.5554416 \\ \mathrm{~F} & -4.7918752 & 0.3278495 & 1.9625897 \\ \mathrm{~F} & -3.2137094 & -4.1344876 & 1.7774505 \\ \mathrm{~F} & -1.4181965 & 3.0409459 & -3.5122560 \\ \mathrm{~F} & -2.0563660 & -1.5749032 & -4.3697180 \\ \mathrm{~F} & 4.6007973 & -3.6740746 & 0.3672052 \\ \mathrm{~F} & -5.1836314 & -2.3503869 & 2.4301318 \\ \mathrm{~F} & -2.2511103 & 1.0368837 & -5.1903508 \\ \mathrm{~S} & 0.3929230 & 0.9935967 & 1.4294825 \\ \mathrm{C} & 1.9621029 & 1.6376245 & 0.9629485 \\ \mathrm{C} & 2.7241212 & 2.2168816 & 1.9908864 \\ \mathrm{C} & 2.4177017 & 1.6800140 & -0.3621335 \\ \mathrm{C} & 3.9180784 & 2.8654556 & 1.6857125 \\ \mathrm{H} & 2.3797802 & 2.1505130 & 3.0184163 \\ \mathrm{C} & 3.6127616 & 2.3234716 & -0.6545498 \\ \mathrm{H} & 1.8320807 & 1.2334835 & -1.1555546 \\ \mathrm{C} & 4.3546079 & 2.9376142 & 0.3622522 \\ \mathrm{H} & 4.5013482 & 3.3201428 & 2.4805958 \\ \mathrm{H} & 3.9615823 & 2.3656839 & -1.6819396 \\ \mathrm{H} & 5.2826601 & 3.4475528 & 0.1227489 \\ \mathrm{C} & 2.4561514 & 5.5010223 & 0.2680659 \\ \mathrm{C} & 1.2435377 & 4.9028170 & 0.2910980 \\ \mathrm{H} & 3.0061818 & 5.6898225 & 1.1852929 \\ \mathrm{H} & 2.9081957 & 5.8357404 & -0.6596416 \\ \mathrm{H} & 0.7054133 & 4.7283903 & -0.6377990 \\ \mathrm{C} & 0.6268042 & 4.4836176 & 1.5088825 \\ \mathrm{C} & -0.6074155 & 3.8420670 & 1.6060531 \\ \mathrm{H} & 1.1941065 & 4.6528152 & 2.4245846 \\ \mathrm{H} & -1.2433039 & 3.8910280 & 0.7203562 \\ \mathrm{H} & -0.2144524 & 2.5441037 & 1.4153268 \\ \mathrm{C} & -1.3290429 & 3.7409908 & 2.9380728 \\ \mathrm{H} & -1.8893257 & 4.6619862 & 3.1341454 \\ \mathrm{H} & -2.0397016 & 2.9101622 & 2.9346379 \\ \mathrm{H} & -0.6208442 & 3.5898579 & 3.7586082\end{array}$

mTSAB (TS for 4-protination of $\mathbf{m} \mathbf{1}$ )

60

Energy $=-3035.589463794$

$\begin{array}{lrrr}\text { B } & -1.2751175 & 0.2323802 & 0.6032033 \\ \text { C } & -0.7048538 & -1.0666306 & 1.3973336 \\ \text { C } & -1.9169037 & 1.4288451 & 1.5132113 \\ \text { C } & -2.3726776 & -0.0725390 & -0.5685750 \\ \text { C } & -0.7406843 & -2.3685476 & 0.8884219 \\ \text { C } & 0.0562670 & -0.9319054 & 2.5658809\end{array}$




\begin{tabular}{|c|c|c|c|}
\hline C & -2.0253354 & 2.7238563 & 0.9985348 \\
\hline & -2.5083506 & 1.2588018 & 2.7684193 \\
\hline & -2.3554003 & 0.3165910 & -1.9063418 \\
\hline & -3.5548697 & -0.7163908 & -0.1825731 \\
\hline & -0.1020979 & -3.4520487 & 1.4866301 \\
\hline & 0.7034097 & -1.9857978 & 3.1987604 \\
\hline ' & -2.6303442 & 3.7838105 & 1.6639642 \\
\hline$C$ & -3.1301903 & 2.2899384 & 3.4693123 \\
\hline C & -3.3998961 & 0.0759657 & -2.7988872 \\
\hline 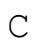 & -4.6212982 & -0.9778037 & -1.0327119 \\
\hline$C$ & 0.6250057 & -3.2631565 & 2.6542474 \\
\hline C & -3.1898881 & 3.5642685 & 2.9177770 \\
\hline 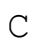 & -4.5445632 & -0.5764341 & -2.3627063 \\
\hline$F$ & -1.3963524 & -2.6500386 & -0.2641845 \\
\hline $\mathrm{F}$ & 0.2351956 & 0.2898518 & 3.1263183 \\
\hline F & -1.5263514 & 2.9983578 & -0.2366577 \\
\hline $\mathrm{F}$ & -2.5175171 & 0.0514374 & 3.3798424 \\
\hline$F$ & -1.2891448 & 0.9657901 & -2.4384472 \\
\hline 7 & -3.6948490 & -1.1344414 & 1.0998133 \\
\hline $\bar{T}$ & -0.1729165 & -4.6797292 & 0.9342141 \\
\hline$F$ & 1.4231749 & -1.7802116 & 4.3206281 \\
\hline$F$ & -2.6875565 & 5.0099867 & 1.1070316 \\
\hline F & -3.6784427 & 2.0622640 & 4.6800191 \\
\hline $\mathrm{F}$ & -3.3056341 & 0.4776537 & -4.0830158 \\
\hline F & -5.7227958 & -1.6140593 & -0.5863259 \\
\hline $\mathrm{F}$ & 1.2536985 & -4.2950875 & 3.2428682 \\
\hline 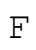 & -3.7851426 & 4.5684231 & 3.5839334 \\
\hline$F$ & -5.5620135 & -0.8141381 & -3.2083463 \\
\hline S & 0.5402935 & 0.9747726 & 0.0199671 \\
\hline C & 1.4913642 & -0.4402841 & -0.4936339 \\
\hline $\mathrm{C}$ & 2.6135276 & -0.7929310 & 0.2639420 \\
\hline $\mathrm{C}$ & 1.1499813 & -1.1745973 & -1.6346963 \\
\hline C & 3.3916574 & -1.8838955 & -0.1198859 \\
\hline H & 2.8693017 & -0.2144662 & 1.1459752 \\
\hline C & 1.9335590 & -2.2638981 & -2.0114518 \\
\hline+ & 0.2817980 & -0.8966084 & -2.2204822 \\
\hline $\mathrm{C}$ & 3.0536027 & -2.6195590 & -1.2571386 \\
\hline $\mathrm{H}$ & 4.2605154 & -2.1587314 & 0.4706878 \\
\hline $\mathrm{H}$ & 1.6675652 & -2.8352216 & -2.8958566 \\
\hline $\mathrm{H}$ & 3.6607590 & -3.4690895 & -1.5550674 \\
\hline $\mathrm{C}$ & 1.3424672 & 2.4035746 & -2.5358990 \\
\hline C & 2.7242881 & 2.3331096 & -2.4029612 \\
\hline $\mathrm{H}$ & 0.8645843 & 1.7423646 & -3.2562257 \\
\hline $\mathrm{H}$ & 0.8572082 & 3.3658597 & -2.3820547 \\
\hline $\mathrm{H}$ & 3.2356247 & 3.1187470 & -1.8466572 \\
\hline $\mathrm{C}$ & 3.5212537 & 1.2420795 & -2.8472115 \\
\hline C & 4.8506126 & 1.1700527 & -2.5832124 \\
\hline $\mathrm{H}$ & 3.0349240 & 0.4419062 & -3.4026511 \\
\hline $\mathrm{H}$ & 5.3078323 & 1.9822178 & -2.0170734 \\
\hline $\mathrm{H}$ & 0.8866145 & 1.8010636 & -1.3881157 \\
\hline $\mathrm{C}$ & 5.7399810 & 0.0531469 & -2.9937331 \\
\hline $\mathrm{H}$ & 6.5848730 & 0.4332131 & -3.5833514 \\
\hline
\end{tabular}




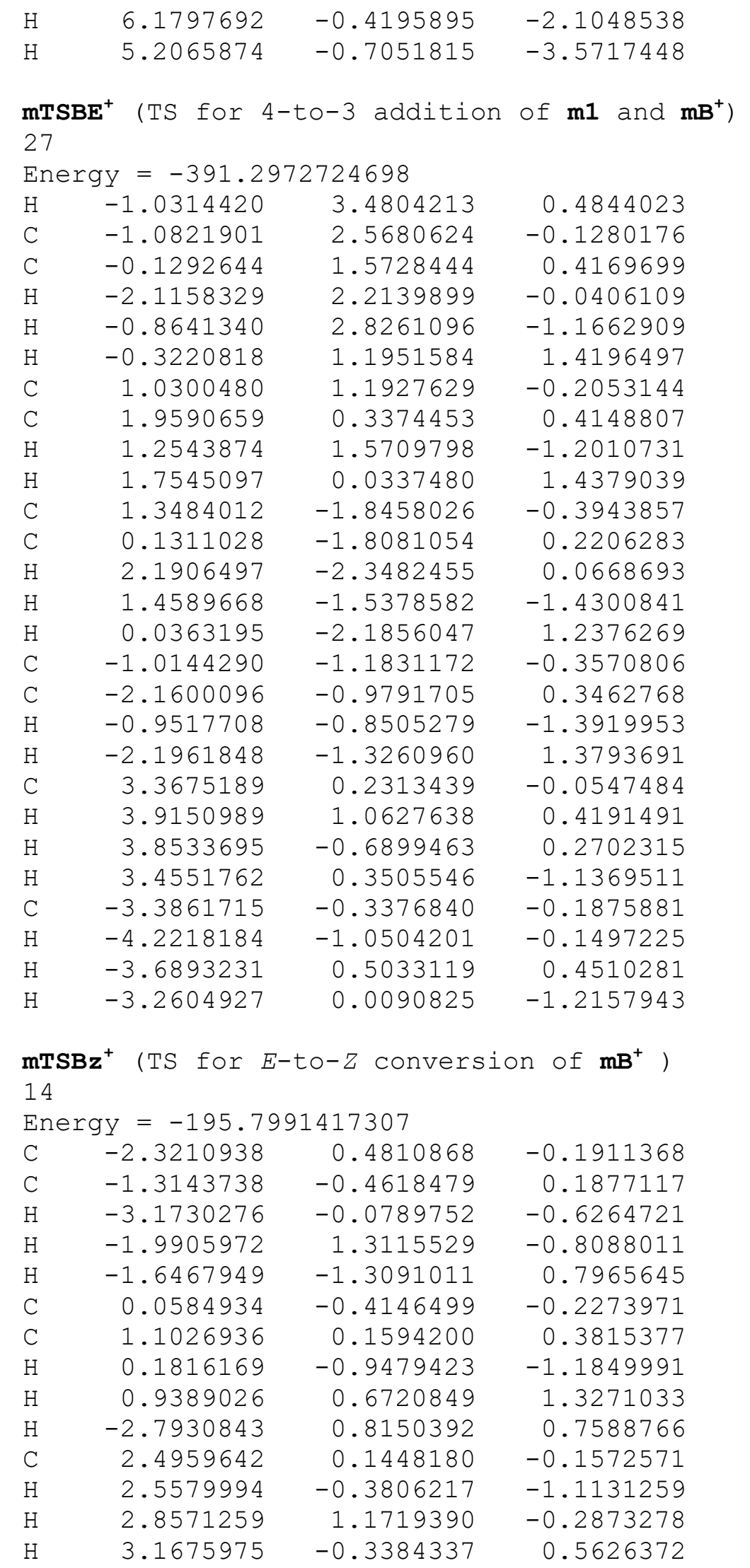


p1 $\mathrm{PhCH}_{2} \mathrm{CH}_{2} \mathrm{CH}=\mathrm{CHCH}=\mathrm{CH}_{2}$ (1-phenethyl 1,3-diene)

26

Energy $=-465.9629856669$

$\begin{array}{lrrr}\mathrm{C} & -4.2835044 & -0.1555600 & -0.1411148 \\ \mathrm{C} & -2.9804882 & 0.1763468 & -0.1379549 \\ \mathrm{H} & -4.6169160 & -1.1431776 & 0.1712499 \\ \mathrm{H} & -5.0485506 & 0.5491920 & -0.4526421 \\ \mathrm{H} & -2.6826617 & 1.1759080 & -0.4577301 \\ \mathrm{C} & -1.9100247 & -0.7144578 & 0.2730509 \\ \mathrm{C} & -0.6063464 & -0.3789063 & 0.2671635 \\ \mathrm{H} & -2.2017986 & -1.7129087 & 0.6044775 \\ \mathrm{H} & -0.3241319 & 0.6229092 & -0.0631977 \\ \mathrm{C} & 0.5066747 & -1.2731761 & 0.7153621 \\ \mathrm{C} & 1.2540026 & -0.7032576 & 1.9514682 \\ \mathrm{H} & 1.2400841 & -1.3963648 & -0.0939363 \\ \mathrm{H} & 0.1170307 & -2.2690452 & 0.9565054 \\ \mathrm{H} & 1.6469259 & 0.2895527 & 1.7034755 \\ \mathrm{H} & 0.5351372 & -0.5783093 & 2.7690256 \\ \mathrm{C} & 2.3767327 & -1.6127537 & 2.3869534 \\ \mathrm{C} & 2.1417426 & -2.6461524 & 3.3038881 \\ \mathrm{C} & 3.6623101 & -1.4805836 & 1.8455248 \\ \mathrm{C} & 3.1611332 & -3.5264756 & 3.6695158 \\ \mathrm{H} & 1.1498345 & -2.7590420 & 3.7360436 \\ \mathrm{C} & 4.6859868 & -2.3578450 & 2.2078312 \\ \mathrm{H} & 3.8625699 & -0.6807519 & 1.1356871 \\ \mathrm{C} & 4.4378987 & -3.3858898 & 3.1204011 \\ \mathrm{H} & 2.9592815 & -4.3202999 & 4.3836818 \\ \mathrm{H} & 5.6774884 & -2.2377541 & 1.7792562 \\ \mathrm{H} & 5.2333978 & -4.0690673 & 3.4043370 \\ & & & \end{array}$

$\mathrm{pBa}^{+}$(1-protonated $\mathbf{p} 1$ with strong $\mathrm{Ph}$...allyl cation interaction) 27

Energy $=-466.3810971459$

$\begin{array}{lrrr}\mathrm{C} & 0.6090953 & 0.2210353 & -0.3441734 \\ \mathrm{C} & -0.0619882 & -0.9237032 & -0.0013841 \\ \mathrm{H} & 0.6014287 & 1.0863063 & 0.3099756 \\ \mathrm{H} & 1.0909303 & 0.3301849 & -1.3101916 \\ \mathrm{H} & -0.0701259 & -1.7763953 & -0.6747244 \\ \mathrm{C} & -0.6426188 & -1.0526825 & 1.2617363 \\ \mathrm{C} & -1.3673461 & -2.2400881 & 1.7490911 \\ \mathrm{H} & -0.6581491 & -0.1704509 & 1.8995499 \\ \mathrm{H} & -2.4173415 & -1.9377920 & 1.8907505 \\ \mathrm{H} & -1.3377846 & -3.0498613 & 1.0140959 \\ \mathrm{C} & -0.8410995 & -2.7164957 & 3.1264283 \\ \mathrm{C} & 0.5980522 & -3.2546542 & 2.9902236 \\ \mathrm{H} & -1.5007030 & -3.4951405 & 3.5179372 \\ \mathrm{H} & -0.8685475 & -1.8777513 & 3.8300930 \\ \mathrm{H} & 0.9888080 & -3.4583082 & 3.9957464 \\ \mathrm{H} & 0.5940196 & -4.2008239 & 2.4406992 \\ \mathrm{C} & 1.4883314 & -2.2681994 & 2.2904636 \\ \mathrm{C} & 2.1799095 & -2.6206314 & 1.1139212\end{array}$




$\begin{array}{lrrr}\mathrm{C} & 1.6471222 & -0.9585133 & 2.8066101 \\ \mathrm{C} & 2.9135028 & -1.6740674 & 0.4206330 \\ \mathrm{H} & 2.0997629 & -3.6360928 & 0.7369357 \\ \mathrm{C} & 2.3980578 & -0.0132317 & 2.1227220 \\ \mathrm{H} & 1.1749767 & -0.6964232 & 3.7491881 \\ \mathrm{C} & 2.9883279 & -0.3494573 & 0.8985231 \\ \mathrm{H} & 3.4175816 & -1.9413940 & -0.5026505 \\ \mathrm{H} & 2.5033591 & 0.9906812 & 2.5209221 \\ \mathrm{H} & 3.5699157 & 0.3883250 & 0.3545340\end{array}$

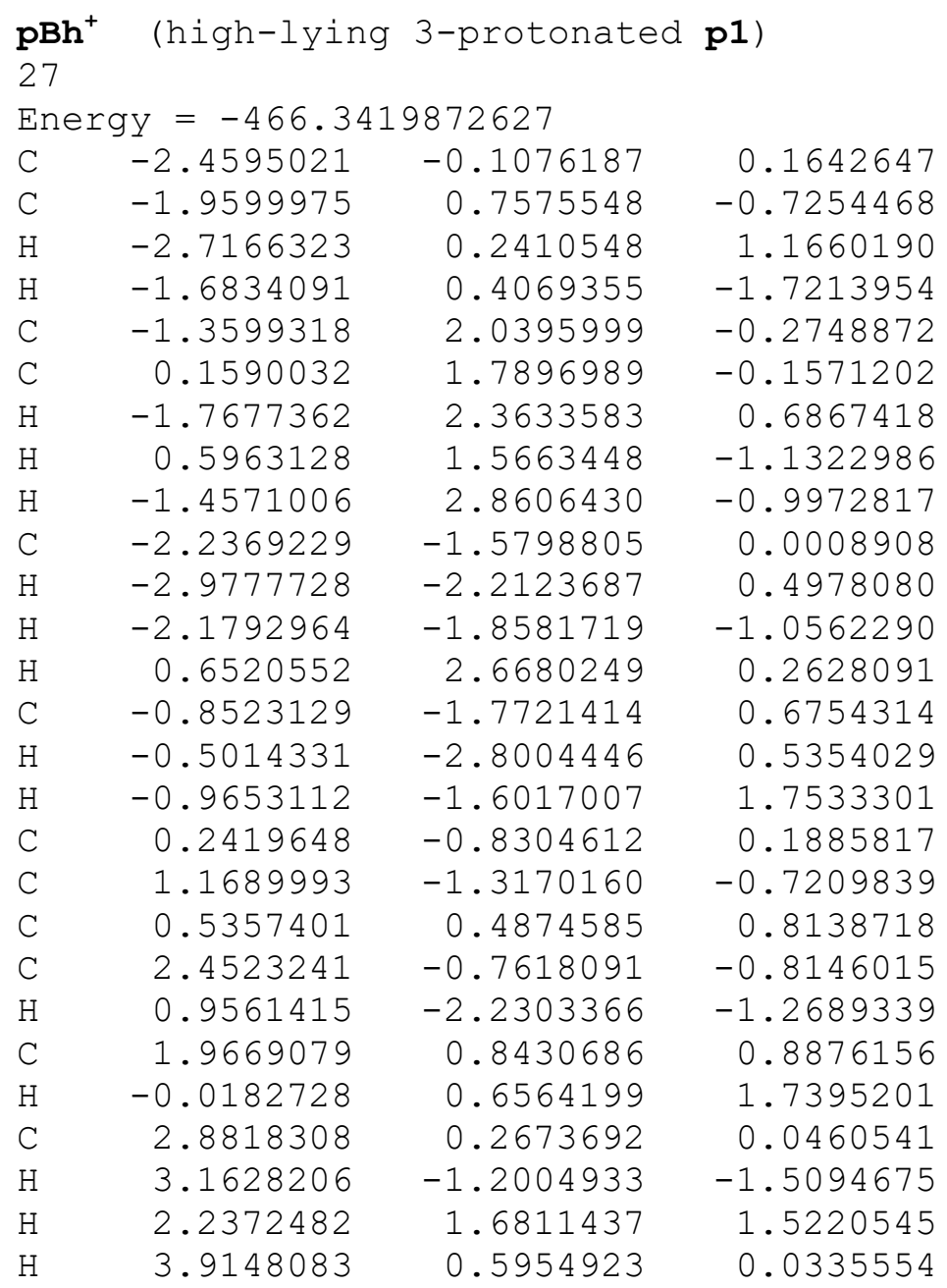

pB $^{+}$(4-protonated $\mathbf{p} 1$, missing channel in experiment)
27
$\begin{array}{lccc}\text { Energy }=-466.3781843259 \\ \text { C } & -1.3872657 & 1.2199494 & 0.1088612 \\ \text { C } & -1.4400442 & 0.2150040 & 1.1699980 \\ \text { H } & -0.9059944 & 0.8676641 & -0.8033493 \\ \text { H } & -0.8824630 & 2.1207310 & 0.4923519 \\ \text { H } & -1.9222293 & 0.5016143 & 2.1045533 \\ \text { C } & -0.9399106 & -1.0700481 & 1.0776650 \\ \text { C } & -1.0833666 & -1.9302716 & 2.1566296 \\ \text { H } & -0.4569905 & -1.4103509 & 0.1651745\end{array}$




$\begin{array}{rrrr}\mathrm{H} & -1.5694906 & -1.5412513 & 3.0511458 \\ \mathrm{H} & -2.4124645 & 1.5610734 & -0.1044728 \\ \mathrm{C} & -0.5564666 & -3.2844023 & 2.2093784 \\ \mathrm{C} & 0.8238456 & -3.2621320 & 3.0217157 \\ \mathrm{H} & -0.3626069 & -3.6958266 & 1.2172418 \\ \mathrm{H} & -1.2267762 & -3.9349977 & 2.7784520 \\ \mathrm{H} & 0.6473264 & -2.8079154 & 3.9995323 \\ \mathrm{H} & 1.0591977 & -4.3192940 & 3.1707509 \\ \mathrm{C} & 1.9255616 & -2.5593349 & 2.2824121 \\ \mathrm{C} & 2.6369144 & -3.2190387 & 1.2701151 \\ \mathrm{C} & 2.2493092 & -1.2288227 & 2.5797332 \\ \mathrm{C} & 3.6554259 & -2.5649761 & 0.5770013 \\ \mathrm{H} & 2.3979930 & -4.2527277 & 1.0303841 \\ \mathrm{C} & 3.2679516 & -0.5726067 & 1.8888955 \\ \mathrm{H} & 1.7097565 & -0.7092178 & 3.3683111 \\ \mathrm{C} & 3.9729508 & -1.2398031 & 0.8845595 \\ \mathrm{H} & 4.2047883 & -3.0909677 & -0.1984147 \\ \mathrm{H} & 3.5142528 & 0.4554248 & 2.1382401 \\ \mathrm{H} & 4.7681713 & -0.7312719 & 0.3475205\end{array}$




\subsection{References}

[S1] Lishchynskyi, A.; Muniz, K. An Approach to the Regioselective Diamination of Conjugated Di- and Trienes. Chem. - Eur. J. 2012, 18, 2212-2216.

[S2] Hilt, G.; Danz, M. Regioselective Cobalt-catalyzed Diels-Alder Reaction Towards 1, 3disubstituted and 1, 2, 3-trisubstituted Benzene Derivatives. Synthesis. 2008, 14, 22572263.

[S3] Marcum, J. S.; Roberts, C. C.; Manan, R. S.; Cervarich, T. N.; Meek, S. J. Chiral Pincer Carbodicarbene Ligands for Enantioselective Rhodium-Catalyzed Hydroarylation of Terminal and Internal 1,3-Dienes with Indoles. J. Am. Chem. Soc. 2017, 139, 1558015583.

[S4] [a]. Liao, L.; Guo, R.; Zhao, X. Organoselenium-Catalyzed Regioselective C- H Pyridination of 1, 3-Dienes and Alkenes. Angew. Chem., Int. Ed. 2017, 56, 3201-3205. [b]. Sardini, S. R.; Brown, M. K. Catalyst Controlled Regiodivergent Arylboration of Dienes. J. Am. Chem. Soc. 2017, 139, 9823-9826.

[S5] Maishal, T. K.; Mondal, B.; Puranik, V. G.; Wadgaonkar, P. P.; Lahiri, G. K.; Sarkar, A. Synthesis, Structure, Electrochemistry and ROMP-activity of New Ferrocenyl Analog of Grubbs' Metathesis Catalyst. J. Organomet. Chem. 2005, 690, 1018-1027.

[S6] [a]. H. Lebel, D. Guay, V. Paquet, K. Huard, Highly Efficient Synthesis of Terminal Alkenes from Ketones. Org. Lett. 2004, 6, 3047-3050. [b]. Katritzky, A. R.; Denisko, O. V. 1, 2-vs 1, 4-Addition of Acylbenzotriazoles to $\alpha, \beta$-Unsaturated Aldehydes and Ketones. A Novel Route to 3-Alkyl-4, 6-diaryl-3, 4-dihydropyran-2-ones. J. Org. Chem. 2002, 67, 3104-3108. [c]. Liu, C.; Wang, Q. Alkenylation of C(sp3 )-H Bonds by Zincation/Copper-Catalyzed Cross-Coupling with lodonium Salts. Angew. Chem., Int. Ed. 2018, 57, 4727-4731. [d] Qiao, C.; Chen, A.; Gao, B.; Liu, Y.; Huang, H. PalladiumCatalyzed Cascade Double C-N Bond Activation: A New Strategy for Aminomethylation of 1,3-Dienes with Aminals. Chin. J. Chem. 2018, 36, 929-933.

[S7] Bar, R. M.; Kirschner, S.; Nieger, M.; Brase, S. Alkyl and Aryl Thiol Addition to [1.1.1]Propellane: Scope" and Limitations of a Fast Conjugation Reaction. Chem. Eur. J. 2018, 24, 1373-1382. 
[S8] Zhang, D.; Liua, C.; Chenb, S.; Zhanga, J.; Chenga, J.; Miao, M.; Highly Efficient Preparation of Hyperbranched Epoxy Resins by UV-initiated Thiol-ene Click Reaction. Progress in Organic Coatings 2016,101, 178-185.

[S9] Stratmann, O.; Kaiser, B.; Fröhlich, R.; Meyer, O.; Hoppe, D. The Configurational Stability of an Enantioenricheda-Thiobenzyllithium Derivative and the Stereochemical Course of Its Electrophilic Substitution Reactions ; Synthesis of Enantiomerically Pure, Tertiary Benzylic Thiols. ${ }^{[1,2]}$ Chem. Eur. J. 2001, 7, 423.

[S10] Tan, J.; Zheng, T.; Xu, K.; Liu, C. Aryne Triggered [2,3]- Sigmatropic Rearrangement of Allyl and Propargyl Thioethers. Org. Biomol. Chem. 2017, 15, 4946-4950.

[S11] Saito, K.; Kondo, K.; Akiyama, T. $\mathrm{B}\left(\mathrm{C}_{6} \mathrm{~F}_{5}\right)_{3}$-Catalyzed Hydrodesulfurization Using Hydrosilanes - Metal-Free Reduction of Sulfides. Org. Lett. 2015, 17, 3366-3369.

[S12] TURBOMOLE V7.3, 2018, A Development of University of Karlsruhe and Forschungszentrum Karlsruhe GmbH, 1989-2007, TURBOMOLE GmbH, since 2007; available from http://www.turbomole.com.

[S13] Tao, J.; Perdew, J. P.; Staroverov, V. N.; Scuseria, G. E. Climbing the Density Functional Ladder: Nonempirical Metal-generalized Gradient Approximation Designed for Molecules and Solides. Phys. Rev. Lett. 2003, 91, 146401.

[S14] Grimme, S.; Antony, J.; Ehrlich, S.; Krieg, H. A Consistent and Accurate ab initio Parametrization of Density Functional Dispersion Correction (DFT-D) for the 94 Elements H-Pu. J. Chem. Phys. 2010, 132, 154104-154119.

[S15] Grimme, S.; Ehrlich, S.; Goerigk, L. Effect of the Damping Function in Dispersion Corrected Density Functional Theory. J. Comput. Chem. 2011, 32, 1456-1465.

[S16] Weigend, F.; Haser, M.; Patzelt, H.; Ahlrichs, R. R1-MP2: Optimized Auxiliary Basis Sets and Demonstration of Efficiency. Chem. Phys. Lett. 1998, 294, 143-152.

[S17] Weigend, F.; Ahlrichs, R. Balanced Basis Sets of Split Valance, Triple Zeta Valance Quality and Quadruple Zeta Valance Quality for H to Rn: Design and Assessment of Accuracy. Phys. Chem. Chem. Phys. 2005, 7, 3297-3305.

[S18] Klamt, A.; Schüürmann, G. COSMO: A New Approach to Dielectric Screening in Solvents with Explicit Expressions for the Screening Energy and its Gradient. J. Chem. Soc., Perkin Trans. 2, 1993, 799-805. 
[S19] Eichkorn, K.; Weigend, F.; Treutler, O.; Ahlrichs, R. Auxiliary Basis Sets for Main Row Atoms and Metals and their use to Approximate Coulomb Potentials. Theor. Chem. Acc. 1997, 97, 119-124.

[S20] Weigend, F. Accurate Coulomb-fitting Basis Sets for $\mathrm{H}$ to Rn. Phys. Chem. Chem. Phys. 2006, 8, 1057-1065.

[S21] Deglmann, P.; May, K.; Furche, F.; Ahlrichs, R. Nuclear Second Analytical Derivative Calculations Using Auxiliary Basis Set Expansions. Chem. Phys. Lett. 2004, 384, 103-107.

[S22] Grimme, S. Supramolecular Binding Thermodynamics by Dispersion Corrected Density Functional Theory. Chem. - Eur. J. 2012, 18, 9955-9964.

[S23] Eckert, F.; Klamt, A. Fast Solvent Screening via Quantum Chemistry: COSMO-RS Approach. AlChE J. 2002, 48, 369-385.

[S24] F. Eckert, A. Klamt, CoSMOtherm, Version C3.0, Release 16.01, 2015, COSMOlogic GmbH \& Co., Leverkusen, Germany.

[S25] Zhao, Y.; Truhlar, D. G. Design of Density Functionals that are broadly Accurate for Thermochemistry, Thermochemical Kinetics, and Nonbonded Interaction. J. Phys. Chem. A 2005, 109, 5656-5667.

[S26] Weigend, F.; Furche, F.; Ahlrichs, R. Gaussian Basis Sets of Quadruple Zeta Valance Quality for Atoms H-Kr. J. Chem. Phys. 2003, 119, 12753-12762. 